\title{
Next generation glycan microarray enabled by DNA-coded glycan library and next-generation sequencing (NGS) technology
}

\author{
Maomao Yan ${ }^{1}$, Yuyang Zhu ${ }^{1}$, Xueyun Liu ${ }^{1}$, Yi Lasanajak ${ }^{1,2}$, Jinglin Xiong ${ }^{1}$, Jingqiao Lu ${ }^{3}$, Xi \\ Lin $^{3}$, David Ashline ${ }^{4}$, Vernon Reinhold ${ }^{4}$, David F. Smith ${ }^{1,2}$, and Xuezheng Song ${ }^{1,2 *}$ \\ ${ }^{1}$ Department of Biochemistry, ${ }^{2}$ Emory Comprehensive Glycomics Core, ${ }^{3}$ Department of Otolaryngology, Emory \\ University School of Medicine, Atlanta, GA, USA; ${ }^{4}$ University of New Hampshire, College of Life Sciences and \\ Agriculture, Durham, NH, USA.
}

\begin{abstract}
Interactions of glycans with proteins, cells and microorganisms play important roles in cell-cell adhesion and host-pathogen interaction. Glycan microarray technology, in which multiple glycan structures are immobilized on a single glass slide and interrogated with glycan binding proteins (GBPs), has become an indispensable tool in the study of proteinglycan interactions. Despite its great success, the current format of the glycan microarray requires expensive, specialized instrumentation and labor-intensive assay and image processing procedures, which limit automation and possibilities for high throughput analyses. Furthermore, current microarray is not suitable for assaying interaction with intact cells due to their large size compared to the two dimensional microarray surface. To address these limitations, we developed the Next Generation Glycan Microarray (NGGM) based on artificial DNA-coding of glycan structures. In this novel approach, a glycan library is presented as a mixture of glycans and glycoconjugates, each of which is coded with a unique oligonucleotide sequence (code). The glycan mixture is interrogated by GBPs followed by the separation of unbound coded glycans. The DNA sequences that identify individual bound glycans are quantitatively sequenced (decoded) by powerful next generation sequencing (NGS) technology and copied numbers of the DNA codes represent relative binding specificities of corresponding glycan structures to GBPs. We demonstrate that NGGM generates glycan-GBP binding data that are consistent with that generated in a slide-based glycan microarray. More importantly, the solution phase binding assay is directly applicable to identifying glycan binding to intact cells, which is not generally attempted using glass slide-based glycan microarrays.
\end{abstract}




\section{Methods and Materials \\ Materials}

Streptavidin-coated magnetic beads were purchased from Thermo Fisher Scientific (Cat.\#65001). All the Biotinylated-lectins were purchased from Vector Laboratories. All the DNA oligos were from Integrated DNA Technologies. The DBCO-PEG4-NHS ester was from Conju-Probe (Cat.\# CP-2028). All the blood group related free glycans were purchased from ELicityl. All the blood group related glycan antibodies were from Immucor Inc. All the serum samples were from Innovative Research. All other common chemicals were from Thermo Fisher Scientific, Oakwoods chemicals, Acros or Sigma-Aldrich. Pig kidneys were purchased from Pel-Freez Biologicals. E. coli strain Top10 and BL21 strains were kindly provided by Dr. Bo Liang (Emory university, U.S.A), Acinetobacter baumannii strain 19606, E. coli strain K12 and FimH KO-K12 were kindly provided by Dr. Marcin Grabowicz (Emory university, U.S.A), and Staphylococcus aureus strain (STA) separated from the blood of a septicemic patient was kindly provided by Dr. Sean Stowell (Emory university, U.S.A).

\section{Mass spectrometry}

MALDI-TOF Mass spectrometry was performed as described previously ${ }^{1-2}$. Briefly, liquid samples were loaded onto an anchorchip target plate with matrix. The matrix for DNA or DNA conjugate samples is 3-Hydroxypicolinic acid (HPA) (saturated aqueous solution mixed with $0.1 \mathrm{M}$ ammonium citrate at ratio of $5: 1$ ), and the matrix for other samples is 2,5-dihydroxybenzoic acid (DHB) $(5 \mathrm{mg} / \mathrm{mL}$ in $50 \%$ acetonitrile with $0.1 \%$ trifluoroacetic acid). After crystallization, the plate was loaded into a Bruker Daltonics Ultraflex-II MALDI-TOF/TOF system. Both linear negative ion mode and reflection positive ion mode were used for MS analysis of DNA-related samples and other samples. A lift mode was used for MS/MS analysis.

Electrospray-Ion trap multistage mass spectrometry $\left(\mathrm{MS}^{\mathrm{n}}\right)$ was performed as in previous report ${ }^{3}$. Briefly, AEAB-labelled Fraction 80 was acetylated using acetic anhydride in saturated sodium bicarbonate. Then the fraction was desalted via porous graphitized carbon solid phase extraction. The esters formed during acetylation were hydrolyzed by incubation in $0.01 \mathrm{M}$ sodium hydroxide at room temperature for four hours, followed by vacuum concentration. The samples were then permethylated using spin columns and purified by liquid-liquid extraction with dichloromethane and $0.5 \mathrm{M}$ aqueous sodium chloride. The dried, permethylated samples were dissolved in 1:1 methanol/water for direct infusion. The dissolved samples were placed onto a Triversa Nanomate (Advion, Ithaca, NY, USA) mounted to an LTQ (ThermoFisher, San Jose, CA, USA). Spray parameters on the Nanomate were generally set to $1.6-1.8 \mathrm{kV}$ and 0.3 psi nitrogen gas pressure, and were adjusted to achieve spray currents between $10-150 \mathrm{nA}$. Activation $\mathrm{Q}$ and activation time for CID were left at default values ( 0.25 and $30 \mathrm{~ms}$, respectively). Normalized collision energy was generally set to $35 \%$. Precursor ion selection for $\mathrm{MS}^{\mathrm{n}}$ experiments was performed manually. Precursor $\mathrm{m} / \mathrm{z}$ and isolation windows were generally set to capture an entire isotopic envelope. Signal averaging was performed to achieve suitable spectral quality. Typically, one isolation scan, with collision energy set to 0, was acquired in each CID data file to document the isolated isotopic envelope.

\section{High-performance liquid chromatography analyses}

HPLC analysis was performed on a Shimadzu HPLC CBM-20A system with RF-10Axl fluorescence detector and SPD-20A UV detector. Fluorescence at $330 \mathrm{~nm}$ excitation and $420 \mathrm{~nm}$ emission and UV absorption at $330 \mathrm{~nm}$ was used to detect 2-amino-N-(2aminoethyl)benzamide (AEAB) tag. For reverse-phase HPLC separation, Thermo Scientific porous graphitized carbon (PGC) or Phenomenex C18 column was used. The mobile phases was composed of acetonitrile, milli-Q water and $0.1 \%$ trifluoroacetic acid (TFA). A linear gradient from $1 \%$ to $80 \%$ acetonitrile in either 25 or 35 min was used. For normal-phase HPLC separation, Phenomenex amine column was used. The mobile phases was composed of acetonitrile, milli-Q water and ammonium acetate. A linear gradient from $85 \%$ acetonitrile $/ 50 \mathrm{mM}$ ammonium acetate to $20 \%$ acetonitrile $/ 200 \mathrm{mM}$ ammonium acetate in either 35 or $120 \mathrm{~min}$ was used. 2D-HPLC was performed as $120 \mathrm{~min}$ normal-phase HPLC separation followed by 35 min reverse-phase HPLC separation.

\section{AEAB-conjugation}

$\mathrm{AEAB}$ and free glycan were conjugated and purified as described previously ${ }^{4}$. Briefly, free glycan reacted with $\mathrm{AEAB} \cdot 2 \mathrm{HCl}$ and $\mathrm{NaCNBH} 3$ in DMSO/AcOH (v/v 7/3) at $65^{\circ} \mathrm{C}$ for 2 hours. Then AEAB-conjugated glycan was precipitated by acetonitrile and redissolved in $\mathrm{H}_{2} \mathrm{O}$ followed by reverse-phase HPLC separation (for defined glycan library) or 2D-HPLC separation (for shotgun glycan library).

\section{Oxidative release and separation of $\mathrm{N}$-linked glycans from pig kidneys}

The pig kidney N-linked glycans were released according to the procedure reported previously ${ }^{2,5}$. Briefly, 14 pig kidneys $(\sim 1.6$ $\mathrm{kg}$ ) were homogenized with $6 \mathrm{~L}$ ice-cold water using Waring blender. $3.8 \mathrm{~L} \mathrm{6 \%}$ sodium hypochlorite solution (Pure Bright) was added and the mixture was stirred for $20 \mathrm{~min}$ at room temperature. The mixture was quenched with formic acid so that the final $\mathrm{pH}$ equals 4.8. Then the mixture was centrifuged to remove precipitated material. The supernatant was collected and passed through $10 \mathrm{kD}$ MWCO membrane using a tangential flow filtration system (Synder Filtration). The permeate through the $10 \mathrm{kD}$ membrane was concentrated on a 600-800Da MWCO membrane using the same TFF system. The concentrate was dried by on a rotary evaporator and a portion of the crude product containing reducing $\mathrm{N}$-glycans was subjected to AEAB conjugation. After AEAB conjugation, the reaction mixture was precipitated with acetonitrile and the pellet containing AEAB-conjugated glycans was redissolved in $1 \mathrm{~mL}$ water and desalted using Sephadex G-25 size exclusive chromatography. The collected glycan-containing fraction was then passed through a Sep-Pak C18 cartridge $(10 \mathrm{~g})$ and washed with $100 \mathrm{~mL} 0.1 \mathrm{M}$ acetic acid solution. AEABconjugated glycans were eluted with $100 \mathrm{~mL} 10 \%$ acetonitrile and $0.1 \mathrm{M}$ acetic acid. The elution was lyophilized and re-dissolved

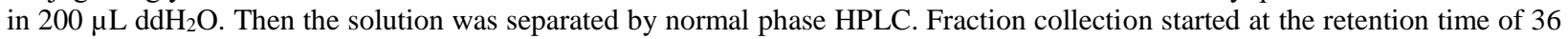


min and ended at $100.2 \mathrm{~min}$. Finally, 46 fractions in total were collected according to the UV absorption. After desalination and

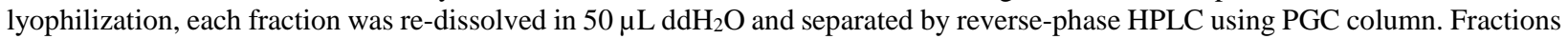
were collected according to the UV absorption. 96 most abundant fractions were selected and quantified according to UV absorption. $1 \mathrm{nmol}$ of each fraction was coded by each DNA code for library construction.

\section{DNA-coded glycan library construction}

Azide-modified double-stranded DNA codes and AEAB-conjugated glycans were used for the preparation of DNA-coded glycans. To obtain azide-modified double strand DNA, same amounts of 5'-azide-modified DNA oligo (IDT Technologies) and its complementary unmodified DNA oligo (IDT Technologies) were annealed together by heating at $95^{\circ} \mathrm{C}$ for 5 min and then cooling down at $0.1{ }^{\circ} \mathrm{C} / \mathrm{s}$ to $10{ }^{\circ} \mathrm{C}$ ) using a PCR instrument. Then AEAB-conjugated glycans and azide-modified DNA codes were conjugated together through a bifunctional crosslinker DBCO-PEG4-NHS (Conju-Probe).

For defined glycan library preparation, 1 eq. AEAB-conjugated glycan was reacted with 2 eq. DBCO-PEG4-NHS in phosphate buffer $\left(50 \mathrm{mM} \mathrm{Na}_{2} \mathrm{HPO}_{4}, \mathrm{pH}=8.5\right)$ at room temperature for 2 hours. The intermediate product glycan-PEG4-DBCO was purified and quantified by reverse-phase HPLC. Then $1 \mu \mathrm{L} 0.1 \mathrm{mM}$ double strand DNA was incubated with $1 \mu \mathrm{L} 0.5 \mathrm{mM}$ AEAB-conjugated glycan-PEG4-DBCO at room temperature. After 3 hours' incubation, $2 \mu \mathrm{L} 0.1 \mathrm{M}$ azidoethanol solution ${ }^{6}$ was added to quench the click-chemistry reaction. The final mixture was stored as DNA-coded glycans at $-20{ }^{\circ} \mathrm{C}$.

For shotgun library, $1 \mathrm{nmol}$ AEAB-conjugated glycan was reacted with 0.2 nmol DBCO-PEG4-NHS in $2 \mu \mathrm{L}$ phosphate buffer (50 $\mathrm{mM} \mathrm{Na}_{2} \mathrm{HPO}_{4}, \mathrm{pH}=8.5$ ) at room temperature for 2 hours. Then $1 \mu \mathrm{L} 0.1 \mathrm{mM}$ double strand DNA was directly added into the mixture. After 3 hours' incubation, $1 \mu \mathrm{L} 0.2 \mathrm{M}$ azidoethanol solution ${ }^{6}$ was added to quench the click-chemistry reaction. 96 fractions were split into two sub-groups for sub-library preparation: fraction 1 to 48 were coded by code 1 to 48 as the sub-library 1 and fraction 49 to 96 were coded by code 1 to 48 as the sub-library 2 . The final mixture was stored as DNA-coded glycans at $20{ }^{\circ} \mathrm{C}$.

\section{Pull-down assay}

For lectin pull-down assay, $1 \mu \mathrm{g}$ or indicated amount of biotinylated lectin was incubated with glycan-DNA mixture (1.25 fmol for each glycan-DNA conjugate) in $10 \mu \mathrm{L} 1 \mathrm{X}$ TSM buffer (20 mM Tris- $\left.\mathrm{HCl}, \mathrm{pH} 7.4150 \mathrm{mM} \mathrm{NaCl}, 2 \mathrm{mM} \mathrm{CaCl}_{2}, 2 \mathrm{mM} \mathrm{MgCl}_{2}\right)$ containing $1 \%$ BSA, $0.05 \%$ TritonX-100 and $100 \mathrm{ng} / \mathrm{mL}$ salmon sperm DNA (Thermo Fisher Scientific) for 2.5 hours. Then $1 \mu \mathrm{L}$ $1 \mathrm{mg} / \mathrm{mL}$ streptavidin-coated magnetic beads (Dynabeads ${ }^{\mathrm{TM}}$ MyOne ${ }^{\mathrm{TM}}$ Streptavidin C1, Thermo Fisher Scientific) was added into the mixture and incubated for 30 minutes. Beads were separated on magnet plate (Low Elution Magnet Plate for 96 Well PCR Plates, E\&K Scientific) and washed with $10 \mu \mathrm{L} \mathrm{1X}$ TSM buffer containing 1\% BSA and 0.05\% TritonX-100 for 3 times. Then

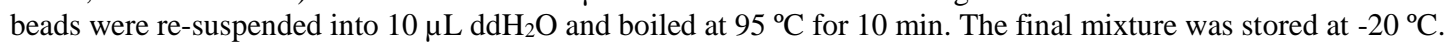

For antibody pull-down assay, $1 \mu \mathrm{L}$ blood type antibody was incubated with glycan-DNA mixture (1.25 fmol for each glycanDNA conjugate) in $10 \mu \mathrm{L} 1 \mathrm{X}$ TSM buffer containing $1 \% \mathrm{BSA}, 0.05 \%$ TritonX-100 and $100 \mathrm{ng} / \mathrm{mL}$ salmon sperm DNA (Thermo Fisher Scientific) for 2.5 hours. Then $1 \mu \mathrm{L} 1 \mathrm{mg} / \mathrm{mL}$ streptavidin-coated magnetic beads (Dynabeads ${ }^{\mathrm{TM}}$ MyOne ${ }^{\mathrm{TM}}$ Streptavidin C1, Thermo Fisher Scientific) which has been pre-incubated with $10 \mu \mathrm{g} / \mathrm{mL}$ biotinylated anti-mouse IgM secondary antibody in $10 \mu \mathrm{L}$ 1X TSM buffer for 2 hours was added into the mixture and incubated for 30 minutes. Beads were separated on magnet plate (Low Elution Magnet Plate for 96 Well PCR Plates, E\&K Scientific) and washed with $10 \mu \mathrm{L} 1 \mathrm{X}$ TSM buffer containing $1 \%$ BSA and $0.05 \%$ TritonX-100 for 3 times. Then beads were re-suspended into $10 \mu \mathrm{L} \mathrm{ddH} \mathrm{O}_{2} \mathrm{O}$ and boiled at $95{ }^{\circ} \mathrm{C}$ for 10 min. The final mixture was stored at $-20^{\circ} \mathrm{C}$.

For human serum pull-down assay, $20 \mu \mathrm{L}$ human serum was incubated with $10 \mu \mathrm{g} / \mathrm{mL}$ biotinylated anti-human IgG or IgM secondary antibody and $1 \mu \mathrm{L} 1 \mathrm{mg} / \mathrm{mL}$ streptavidin-coated magnetic beads in $100 \mu \mathrm{L} 1 \mathrm{X}$ TSM buffer containing $0.05 \%$ TritonX100 for 3 hours. Then beads were separated on a magnet separator and washed with $200 \mu \mathrm{L} 1 \mathrm{X}$ TSM buffer containing $0.05 \%$ TritonX-100. After wash, beads were incubated with glycan-DNA mixture (1.25 fmol for each glycan-DNA conjugate) in $10 \mu \mathrm{L}$ $1 X$ TSM buffer containing $1 \%$ BSA, $0.05 \%$ TritonX-100 and $100 \mathrm{ng} / \mathrm{mL}$ salmon sperm DNA for 2.5 hours. After incubation, beads were separated on magnet plate (Low Elution Magnet Plate for 96 Well PCR Plates, E\&K Scientific) and washed with $10 \mu \mathrm{L} 1 \mathrm{X}$ TSM buffer containing $1 \%$ BSA and $0.05 \%$ TritonX-100 for 3 times. Then beads were re-suspended into $10 \mu \mathrm{L}$ dd $\mathrm{H}_{2} \mathrm{O}$ and boiled at $95^{\circ} \mathrm{C}$ for $10 \mathrm{~min}$. The final mixture was stored at $-20^{\circ} \mathrm{C}$.

For bacteria pull-down assay, $400 \mu \mathrm{L}$ bacteria cultures (OD 1) were spin down at 3,000 g for $1 \mathrm{~min}$. The bacteria pellet were resuspended and incubated with glycan-DNA mixture ( $25 \mathrm{fmol}$ for each glycan-DNA conjugate) in $100 \mu \mathrm{L} 1 \mathrm{X}$ TSM buffer containing $1 \%$ BSA, $0.05 \%$ TritonX-100 and $100 \mathrm{ng} / \mathrm{mL}$ salmon sperm DNA for 1 hours. Then the bacteria cells were separated by centrifugation and washed with $100 \mu \mathrm{L} 1 \mathrm{X}$ TSM buffer containing 1\% BSA and $0.05 \%$ TritonX-100 for 3 times. Then the cells were re-suspended in $100 \mu \mathrm{L} 1 \mathrm{X}$ PBS buffer. $1 \mu \mathrm{L}$ of suspension was used for PCR amplification immediately.

\section{Sample preparation for NGS sequencing}

$1 \mu \mathrm{L}$ supernatant (except for bacteria pull-down assay) or suspension (bacteria pull-down assay) of each sample was subjected to a 20 30-cycles PCR amplification with a pair of primers including two sample coding sequences as indexes for lectin/antibody/serum/bacteria. Comparable amounts of different PCR products of first round amplification were mixed together and purified with PureLink ${ }^{\mathrm{TM}}$ Quick PCR Purification Kit (Thermo Fisher Scientific). After quantification, $10 \mu \mathrm{g}$ DNA mixture was subjected to another round 10-cycles amplification. The second round PCR product was purified again with PureLink ${ }^{\mathrm{TM}}$ Quick PCR Purification Kit (Thermo Fisher Scientific) before submission to NGS sequencing (Admera Health, LLC).

Multiplexing of glycan microarray 
In a NGGM the array is represented as a mixture of soluble DNA-coded glycans in a small vial, and multiplexing is easily accomplished by simply assaying different GBPs in separate tubes or in a multi-well format, which is compatible with automated liquid handling. Since the NGS platform has extremely high capacity for sequencing, it is possible to incorporate multiple DNA-coded glycan microarray analyses in a single NGS run. This can be easily achieved through an "indexing" approach shown in Figure 1 lower panel. For each tube, beads conjugated with a different GBP or cultured cells or cloned microorganisms (samples) can be added and assayed with the DNA-coded glycan library. After incubation and wash, the samples can be transferred to a PCR reaction plate. For each PCR reaction, a different pair of primers are added, of which each primer includes a 3'-region same as the 5'-primer binding region of the glycan-DNA conjugates, a specific index coding region to identify the GBP and a 5'-adaptor binding region compatible with the NGS sequencing system. After reduced cycles of PCR amplification, the amplified DNA from all wells can be pooled together for NGS. We have adapted this multiplexing approach for up to 55 GBP samples in a single NGS analysis using 1M reads, which greatly reduces the time required and cost of each microarray analysis. If NGS analysis of higher capacity (up to $8000 \mathrm{M}$ reads in HiSeq platform) is used, many more samples can be adopted in a single run, which would further reduce the cost of each microarray experiment.

\section{NGS data analysis}

The resulting data file (.gz) was analyzed under the Ubuntu operation system. The target sequences was matched in the data file by python3 software and counted. The numbers was output into an excel file.

For each pull-down assay coded by a unique index pair, the total DNA (50 codes) copy number was normalized to 1 million. After normalization, the copy number of each DNA code was graphed.

For shotgun glycan array, as we only use 50 codes for 96 fractions, so 96 fractions were divided into two subarrays, each subarray contains 48 DNA-coded fractions, one DNA-coded 2-FL and a DNA control. Two subarrays were pulled down separately in different well but simultaneously. After pull-down assay, NGS sequencing and data analysis, the copy numbers of two sub-arrays were combined together after copy number adjustment so that the total copy number of 2-FL (code 49) and a DNA control (code 50) of each subarray equals. Then the total copy number of 96 fractions, 2-FL (code 49) and a DNA control (code 50) was normalized to 1 million. After normalization, the copy number of each fractions was graphed.

\section{Printed glycan microarray analysis}

AEAB-tagged glycans were printed on NHS-activated slides at $100 \mu \mathrm{M}$ in $0.1 \mathrm{M}$ sodium phosphate (pH 8.5) in replicates of four. Biotinylated lectins (Vector Labs) were assayed at $10 \mu \mathrm{g} / \mathrm{mL}$ or indicated concentrations. Cy5-streptavidin (Invitrogen, $1 \mu \mathrm{g} / \mathrm{mL}$ ) was used to detect the bound biotinylated lectins by a fluorescent scanner (Molecular Diagnostics).

\section{Polyacrylamide gel electrophoresis}

8\% PAGE gel was prepared with 0.2 volume $40 \%$ acrylamide/bis-acrylamide (19:1) solution, 0.1 volume 10x TBE buffer, 0.02 volume $10 \%$ ammonium persulfate (APS) and 0.68 volume $\mathrm{ddH}_{2} \mathrm{O}$. DNA was loaded by 0.2 volume 6x DNA Loading Dye (Thermo Scientific). Then the $7 \times 10 \mathrm{~cm}$ gel was run at 150 Volts for $40 \mathrm{~min}$. The resulting gel was stained by Ethidium Bromide (EB) $(0.3 \%$ EB in $0.5 x$ TBE buffer) and photographed by UV detector.

\section{Ethyl esterification and glycan enrichment}

The ethyl esterification was performed similarly as described previously ${ }^{7}$. In brief, $\sim 4$ nmol pig kidney Fraction 80 was dissolved in $1 \mu \mathrm{L}$ and was added to $20 \mu \mathrm{L}$ of ethanol containing $0.25 \mathrm{M}$ 1-Ethyl-3-(3-dimethylaminopropyl) carbodiimide (EDC) and 0.25 M N-Hydroxybenzotriazole (HOBt). The mixture was incubated for 1 hour at room temperature. After incubation, the $20 \mu \mathrm{L}$ acetonitrile $(\mathrm{ACN})$ was added. The mixture was mixed well before subjecting onto glycan enrichment.

For glycan enrichment, $30 \mathrm{mg}$ cellulose microcrystalline bead ( $90 \mu \mathrm{m}$ particle, ACROS Organics) was packed into a bead column

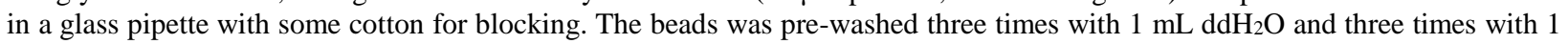
$\mathrm{mL} 85 \% \mathrm{ACN}$. Then the total $40 \mu \mathrm{L}$ mixture from esterification was loaded onto bead column and incubated for $10 \mathrm{~min}$. Then the beads was washed three times with $1 \mathrm{~mL} 85 \%$ ACN containing 1\% TFA and three times with $1 \mathrm{~mL} 85 \%$ ACN. Subsequent elution

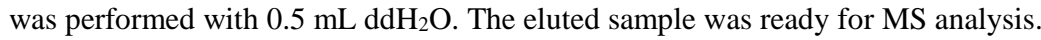

\section{REFERENCES}

(1) Bentzley, C. M.; Johnston, M. V.; Larsen, B. S.; Gutteridge, S., Oligonucleotide sequence and composition determined by matrixassisted laser desorption/ionization. Anal Chem 1996, 68 (13), 2141-6.

(2) Song, X.; Ju, H.; Lasanajak, Y.; Kudelka, M. R.; Smith, D. F.; Cummings, R. D., Oxidative release of natural glycans for functional glycomics. Nat Methods 2016, 13 (6), 528-34.

(3) Ashline, D. J.; Yu, Y.; Lasanajak, Y.; Song, X.; Hu, L.; Ramani, S.; Prasad, V.; Estes, M. K.; Cummings, R. D.; Smith, D. F.; Reinhold, V. N., Structural characterization by multistage mass spectrometry (MSn) of human milk glycans recognized by human rotaviruses. Mol Cell Proteomics 2014, 13 (11), 2961-74.

(4) Song, X.; Xia, B.; Stowell, S. R.; Lasanajak, Y.; Smith, D. F.; Cummings, R. D., Novel fluorescent glycan microarray strategy reveals ligands for galectins. Chem Biol 2009, 16 (1), 36-47. 
(5) Zhu, Y.; Yan, M.; Lasanajak, Y.; Smith, D. F.; Song, X., Large scale preparation of high mannose and paucimannose N-glycans from soybean proteins by oxidative release of natural glycans (ORNG). Carbohydr Res 2018, 464, 19-27.

(6) Baskin, J. M.; Prescher, J. A.; Laughlin, S. T.; Agard, N. J.; Chang, P. V.; Miller, I. A.; Lo, A.; Codelli, J. A.; Bertozzi, C. R., Copperfree click chemistry for dynamic in vivo imaging. Proc Natl Acad Sci U S A 2007, 104 (43), 16793-7.

(7) Reiding, K. R.; Blank, D.; Kuijper, D. M.; Deelder, A. M.; Wuhrer, M., High-throughput profiling of protein N-glycosylation by MALDI-TOF-MS employing linkage-specific sialic acid esterification. Anal Chem 2014, 86 (12), 5784-93. 
A

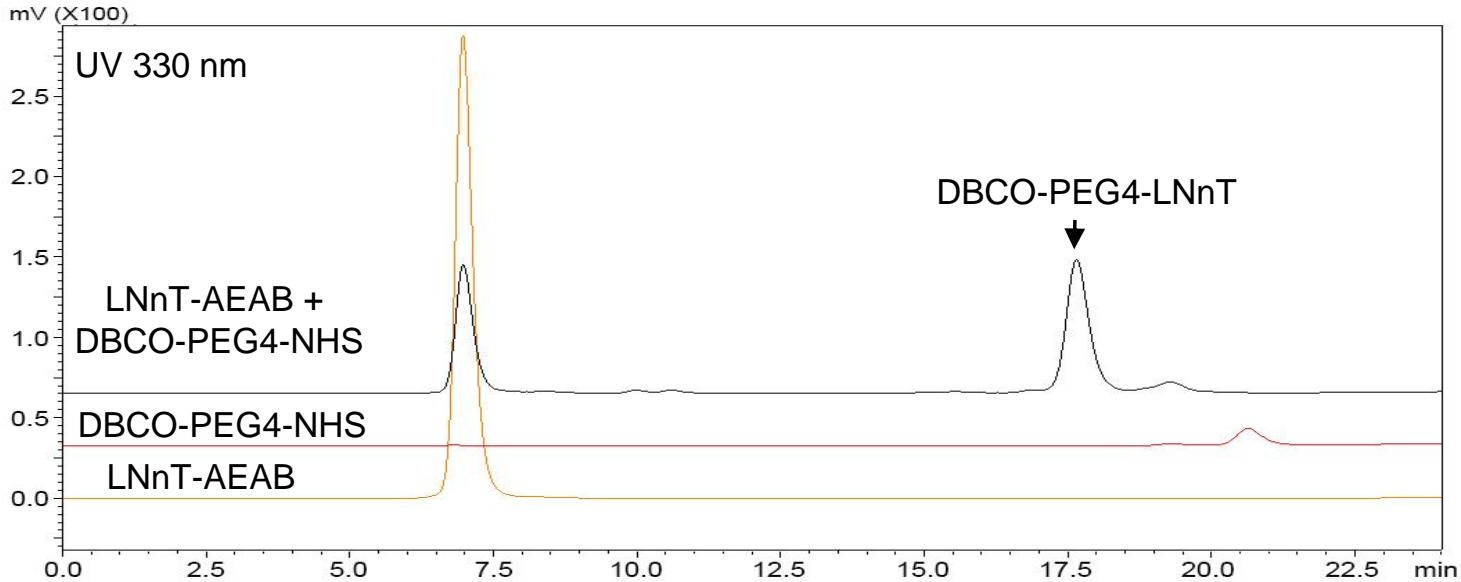

B
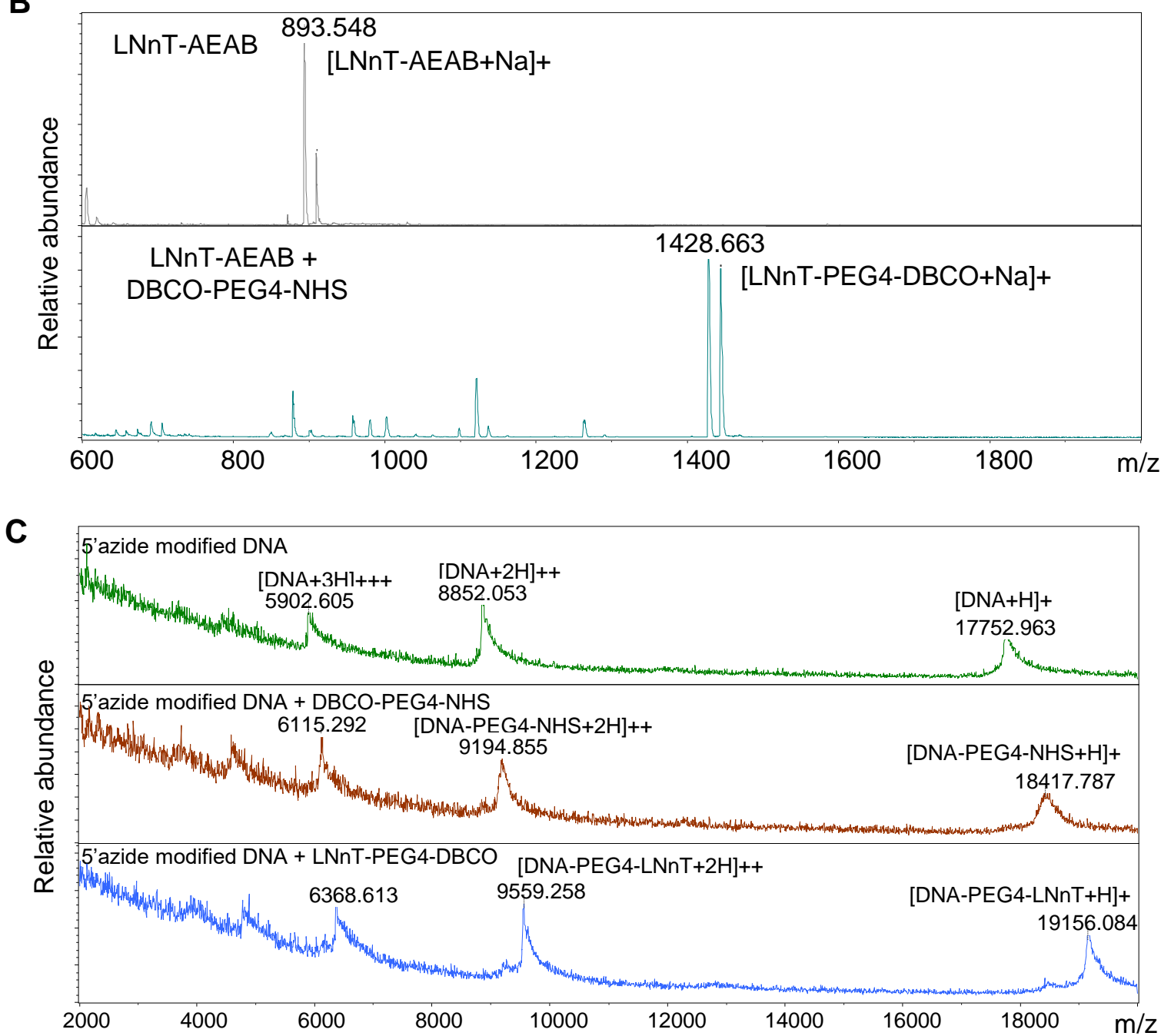

Figure S1. Chemical conjugation of glycan with DNA. (A) Reverse phase C18-HPLC profiles of LNnT-AEAB, DBCO-PEG4-NHS and reaction mixture of LNnT-AEAB and DBCO-PEG4-NHS (from bottom top). UV $330 \mathrm{~nm}$ is used to detect AEAB and related chromophore. (B) MALDI-TOF-MS profiles of LNnT-AEAB and reaction product of LNnT-AEAB and DBCO-PEG4-NHS (from top to bottom). (C) MALDI-TOF-MS profiles of 5'azide modified DNA (top), product of 5'azide modified DNA with DBCOPEG4-NHS, and product of 5'azide modified DNA with DBCO-PEG4-LNnT (from top to bottom). Here Code3s was used as the 5'azide modified DNA. Page-S6 
$1 \bigcirc^{\beta 3} \square^{\beta 3} O^{\beta 4} \mathrm{C}$

$2 \bigcirc^{\beta 4} \square^{\beta 3} \bigcirc^{\beta 4} \bigcirc$

$3 \bigcirc_{a 2}^{\beta 4} \square^{\beta 3} \bigcirc^{\beta 4} \bigcirc$ $\square^{\mathrm{a} 3} \mathrm{Ba}^{\mathrm{B} 2} \square^{\mathrm{a} 4}$

4 $\checkmark \mathrm{a}$

5

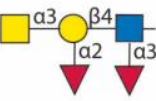

6

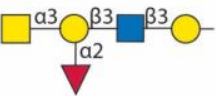

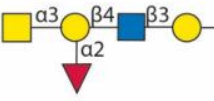

$8 \square^{a 3} \overbrace{\mathrm{la2}}^{\beta 3} \square^{\beta 3} \mathrm{O}^{\beta 4} \bigcirc$

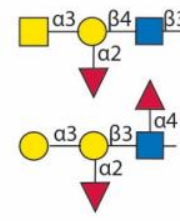

11

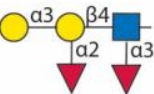

12

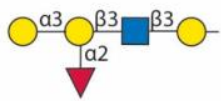

13

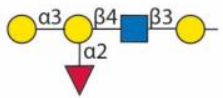

14
$22 \bigcirc^{\alpha 3}\left[O^{\beta 4} \square^{\beta 3}\right]_{2}^{\beta 4} \bigcirc$

15

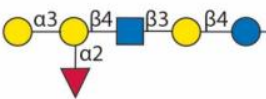

16

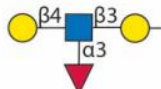

18
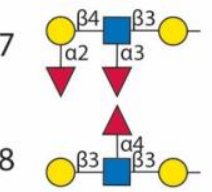

19

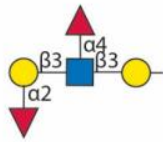

20

$\bigcirc^{\alpha 3} O^{\beta 4} \square^{\beta 3} O^{\beta 4} O$

$21 \bigcirc^{\beta 3} \square^{\beta 3} O^{\alpha 3} O^{\beta 4} O$

$23 \bigcirc^{a 4} \bigcirc^{\beta 4}$

$24 \square \square^{\beta 3} O^{\alpha 4} O^{\beta 4} O$

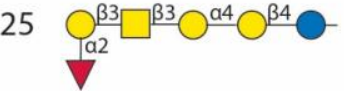

26

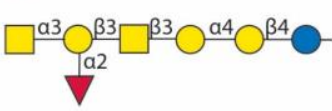

27

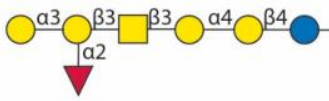

28

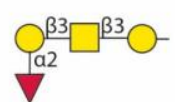

29

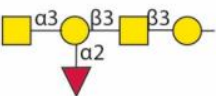

30

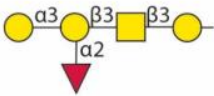

31

$\square^{a 3} \square^{\beta 3} \bigcirc^{\alpha^{4}} \bigcirc^{\beta 4} \bigcirc$

$32 \square \square^{\alpha 3} \square^{\beta 3} O^{\alpha 3} O^{\beta 4} O$

$33 \bigcirc^{a 4} \bigcirc^{\beta 4}$
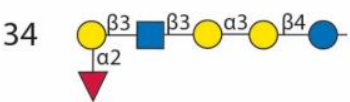

35
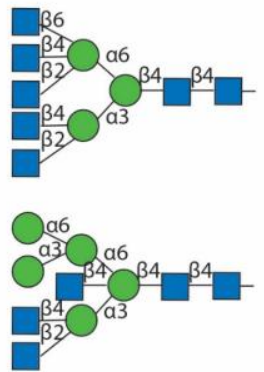

37

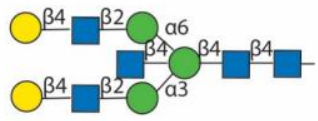

46

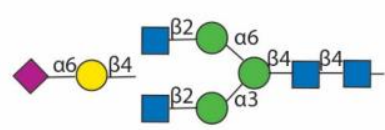

38

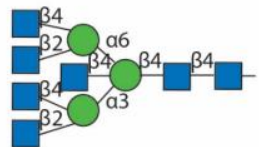

39

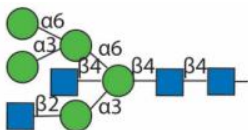

41

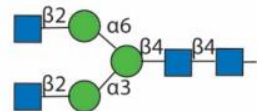

42

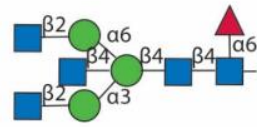

43

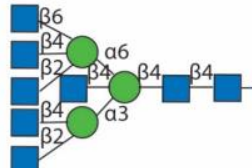

44

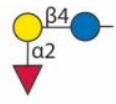

45

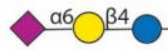

Figure S3. 48 glycan structures used for defined DNA-coded glycan library construction 

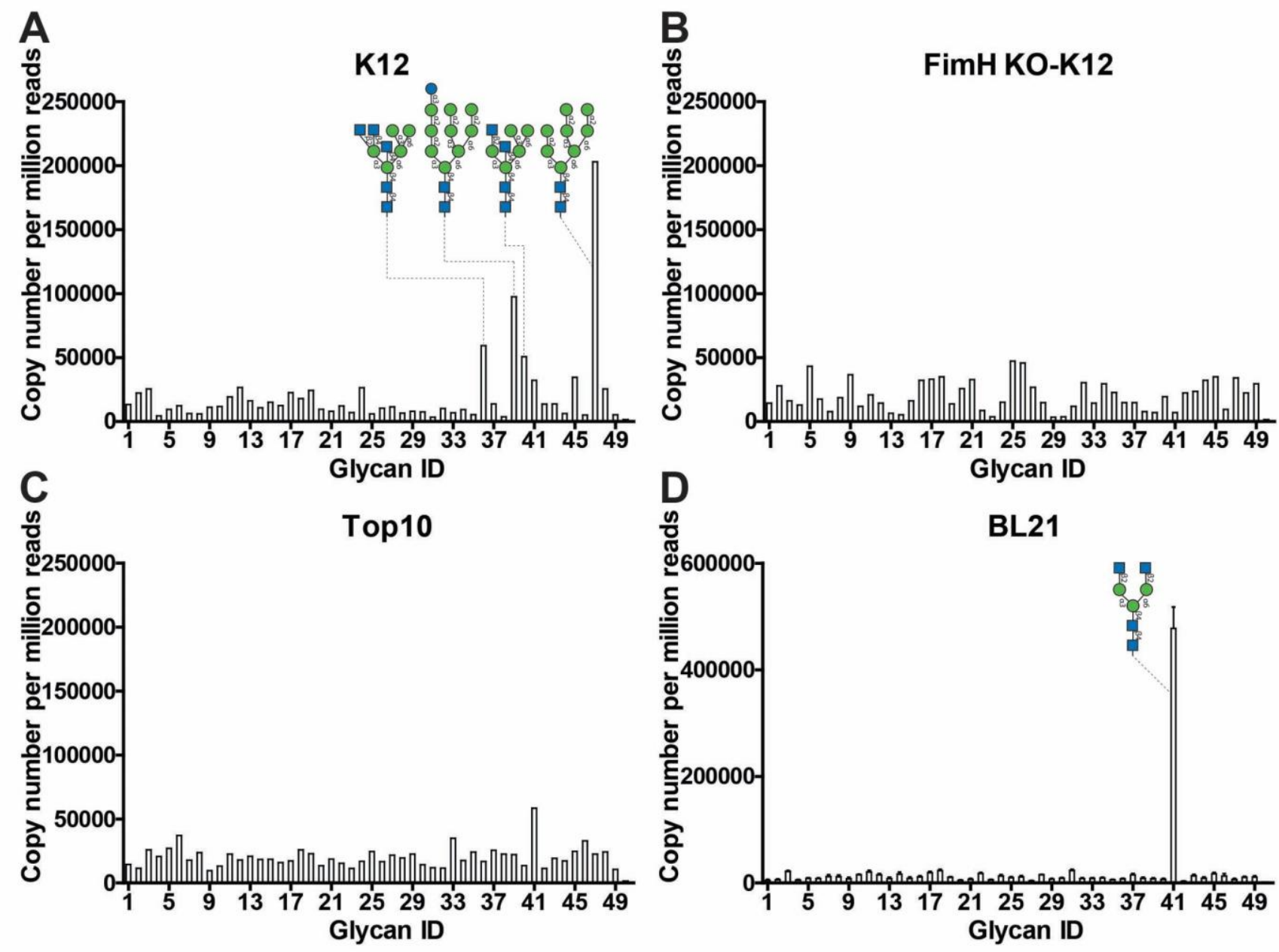

Figure S9. Identification of glycan bindings of 4 E. coli. strains (K12, FimH KO-K12, Top10 and BL21) by NGGM with defined DNA-coded glycan library. Bars in (D) represent means \pm SD from analyses of six independent experiments. 
A
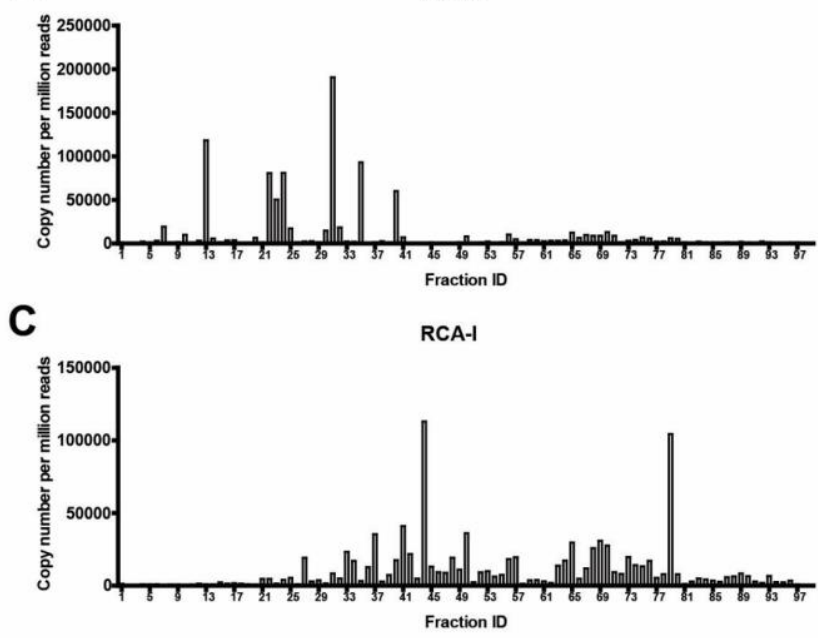

E

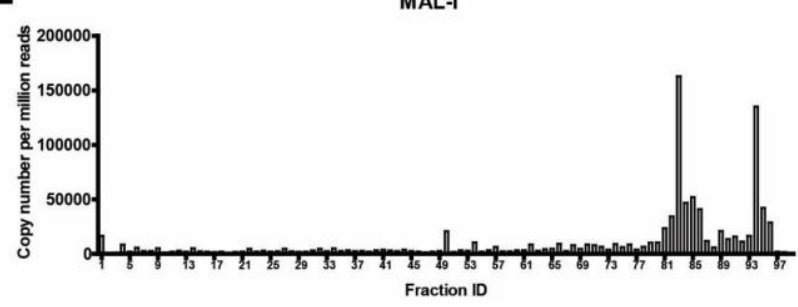

G

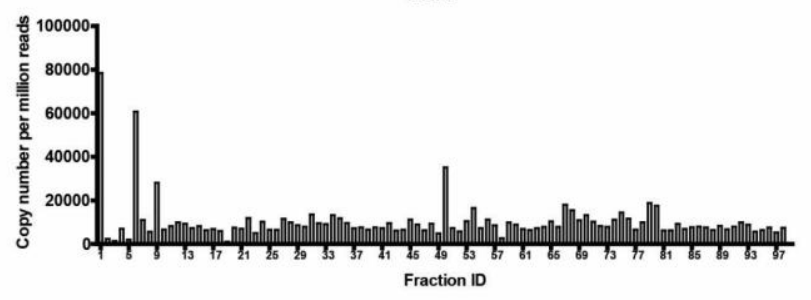

I

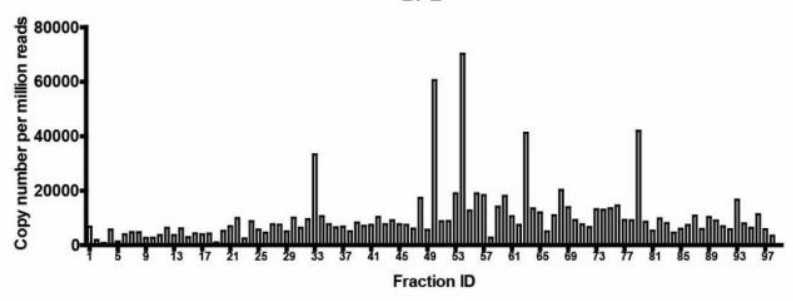

B

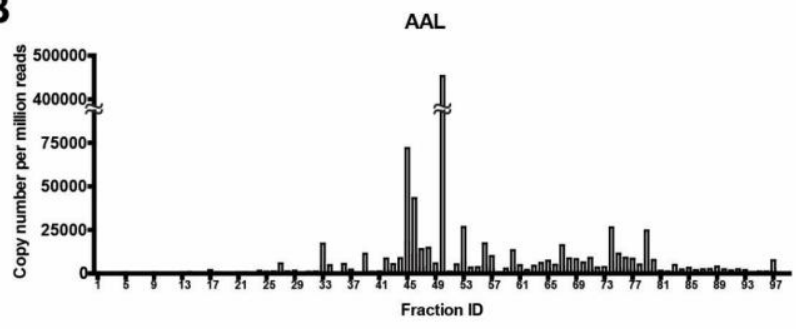

D

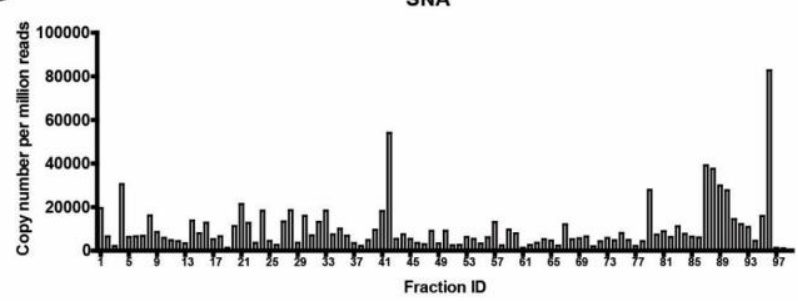

$\mathbf{F}$

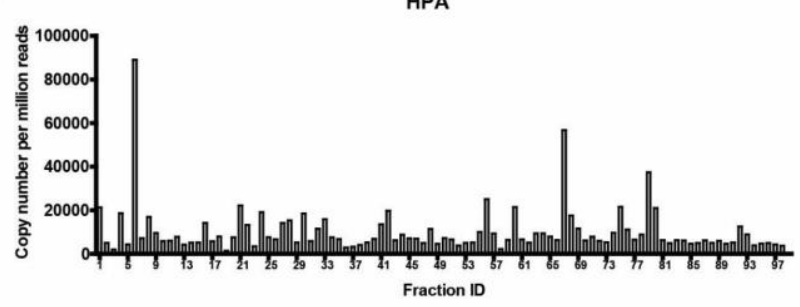

H

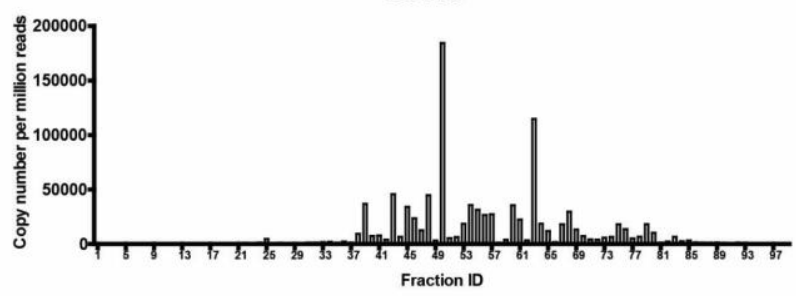

Figure S10. Lectin bindings of pig kidney shotgun glycan library by NGGM. 96 glycan fractions released from pig kidneys were split into two sub-groups for sub-library preparation: fraction 1 to 48 were coded by code 1 to 48 as the sub-library 1 and fraction 49 to 96 were coded by code 1 to 48 as the sublibrary 2. Fraction 97 was DNA-coded 2'-FL and Fraction 98 was DNA only control. $100 \mu \mathrm{g} / \mathrm{ml}$ of indicated biotinylated lectins were incubated with each DNA-coded glycan fraction sub-library $(1.25 \mathrm{fmol}$ for each reaction) for 2.5 hours and absorbed by magnetic beads for $30 \mathrm{~min}$. After wash, the magnetic beads were subjected to PCR amplification and NGS sequencing. The related copy number of each fraction in sublibrary 1 (Fraction 1 48) pulled down by a lectin was calculated by dividing the copy number of each fraction pulled down by blank magnetic beads, and the related copy number of each fraction in sub-library 2 (Fraction 49 96) pulled down by the same lectin was calculated by dividing the copy number of each fraction pulled down by blank magnetic beads the related copy number of 2'-FL plus DNA only control. 
A

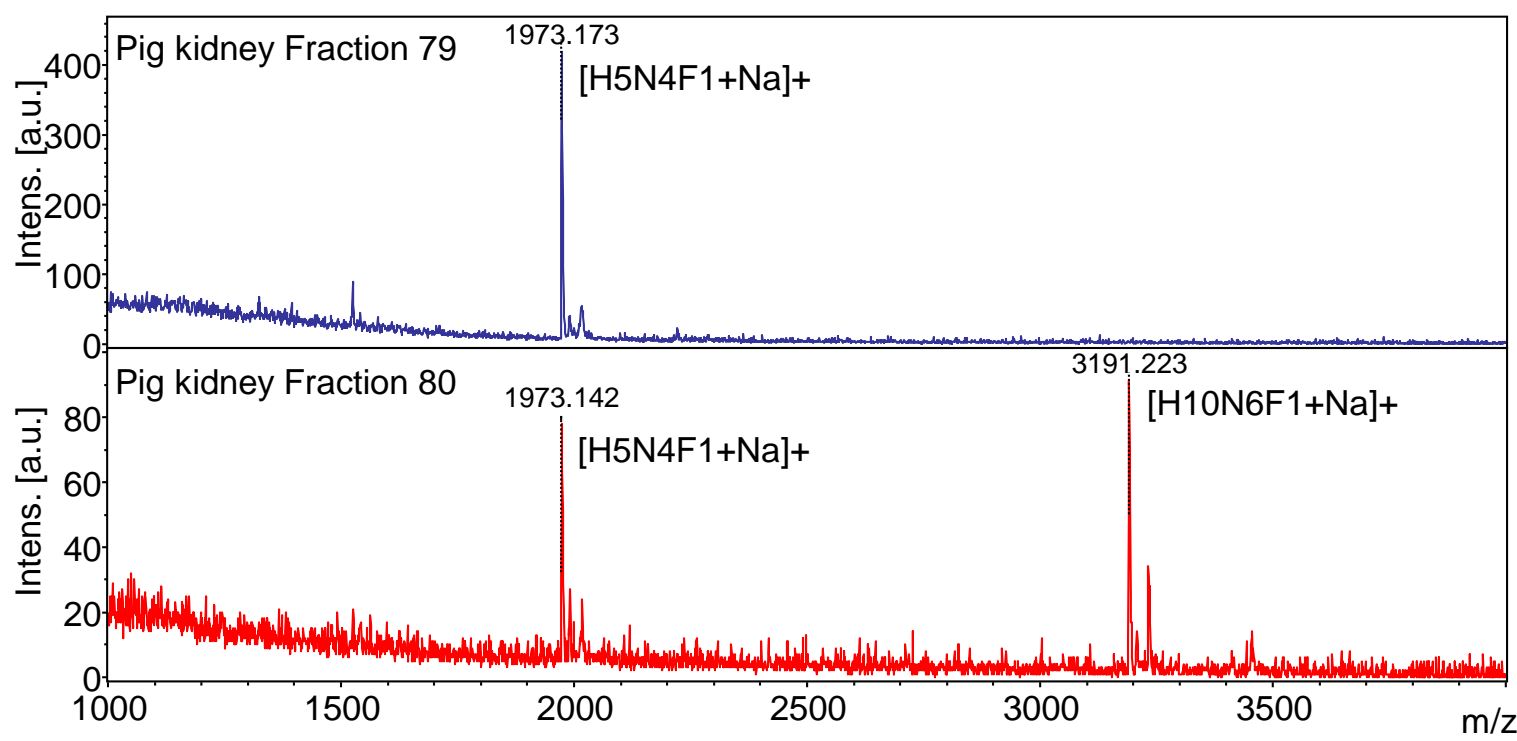

B

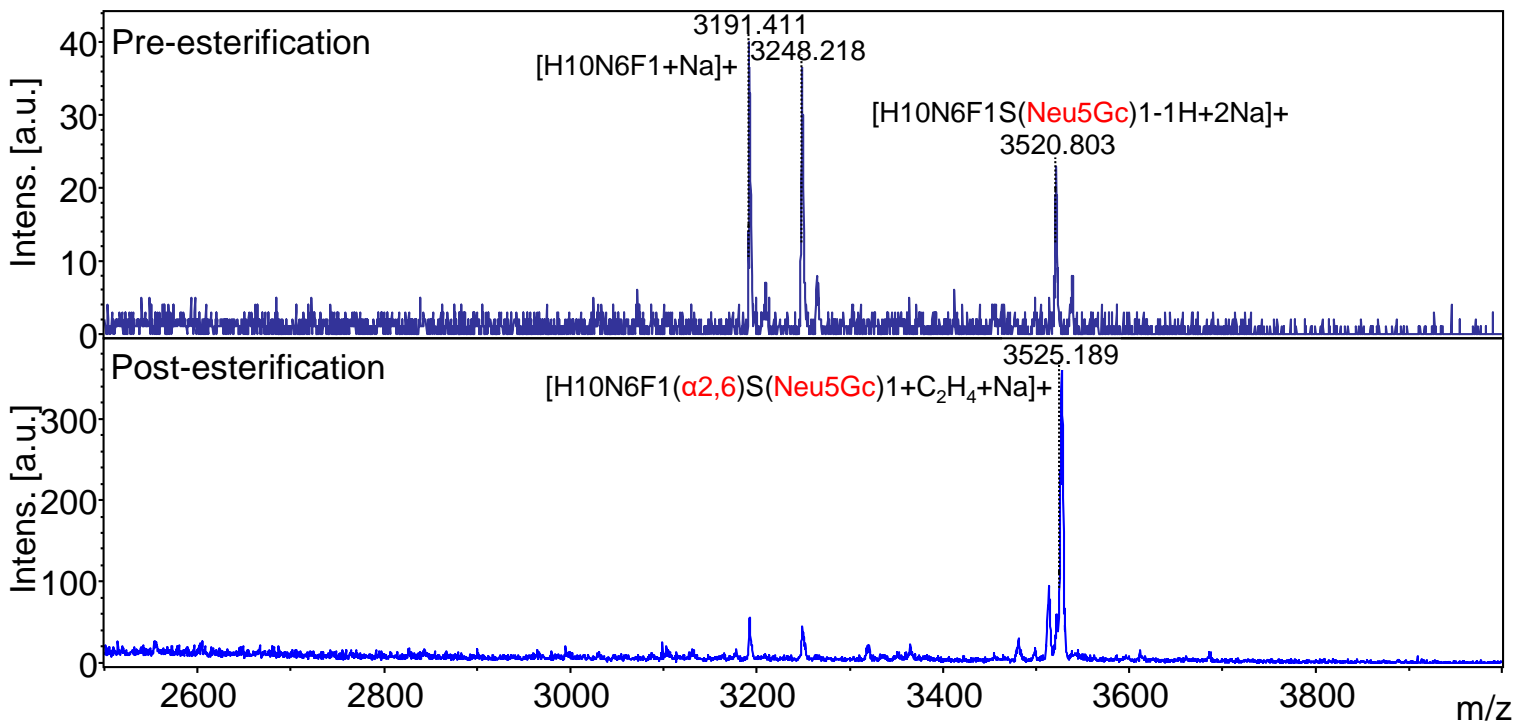

Figure S11. Composition and sialylated linkage determination of Fraction 80 by MALDI-TOF-MS and ethyl esterification. (A) MALDI-TOF positive-mode mass spectrometry of the original pig kidney Fraction 79 (unbound) and Fraction 80 (bound). The corresponding compositions are show. (B) MALDITOF positive-mode mass spectrometry of re-purified pig kidney Fraction 80 before (Pre-esterification) or after ethyl esterification (Post-esterification). This re-purified pig kidney Fraction 80 contains another impurity whose signal on MALDI corresponding to M.W.3248.218. The bound glycan-related compositions are show. 
A

$\mathrm{MS}^{2} \mathrm{~m} / \mathrm{z}^{1489^{3+}}$
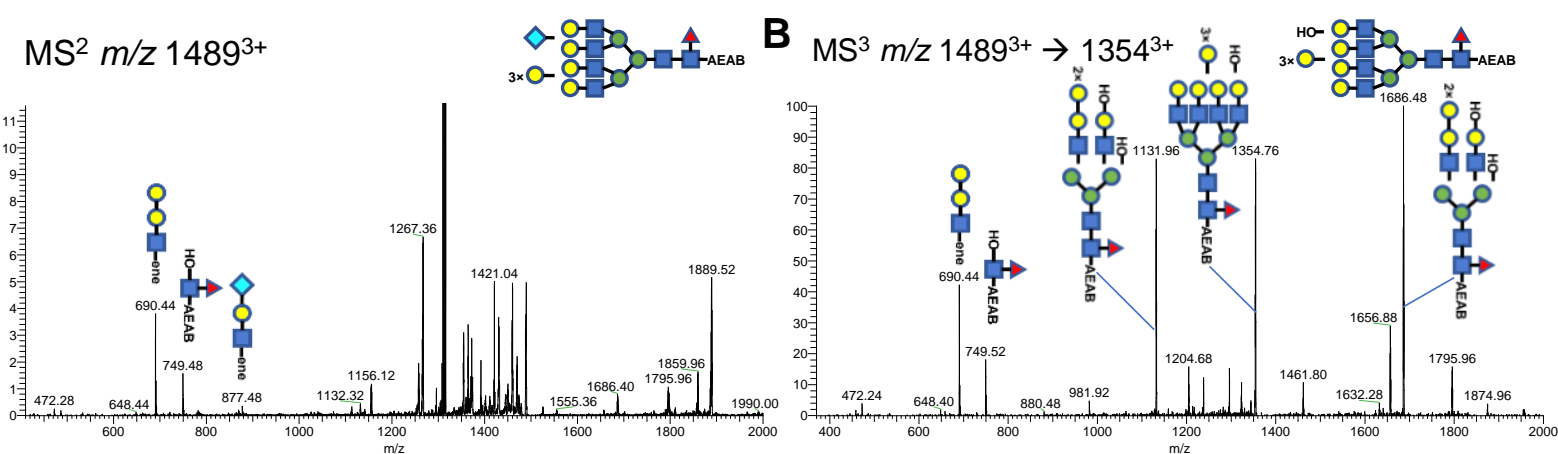

C

$\mathrm{MS}^{3} \mathrm{~m} / \mathrm{z} 1489^{3+} \rightarrow 690^{+}$

D $\mathrm{MS}^{4} \mathrm{~m} / \mathrm{z} 1489^{3+} \rightarrow 690^{+} \rightarrow 445^{+}$

O-O-ene
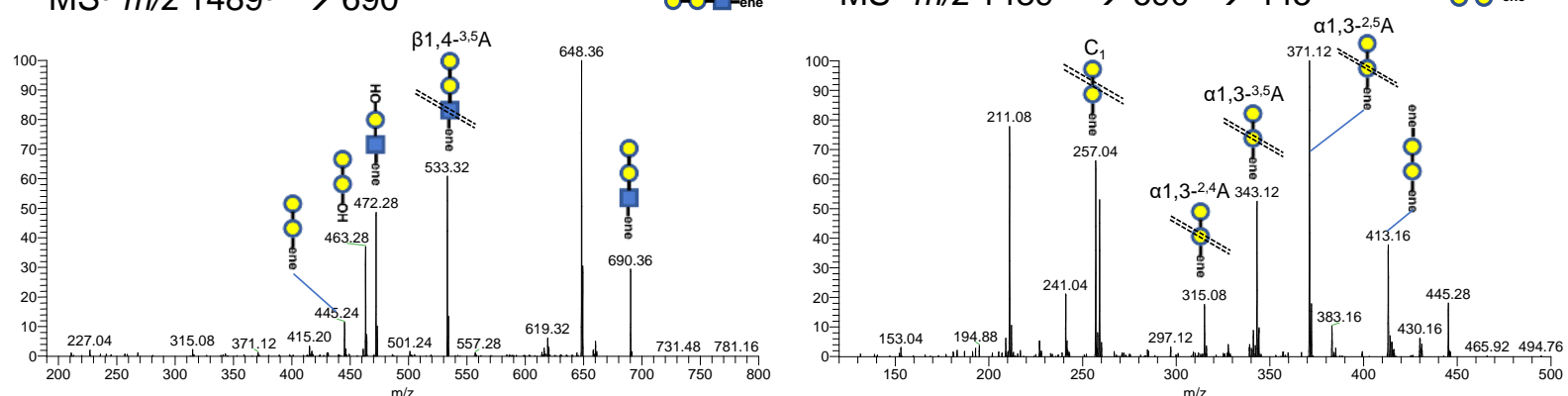

$\mathbf{E}$

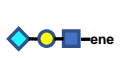

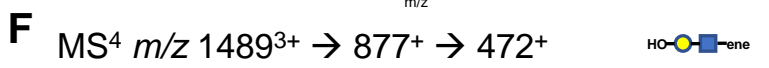
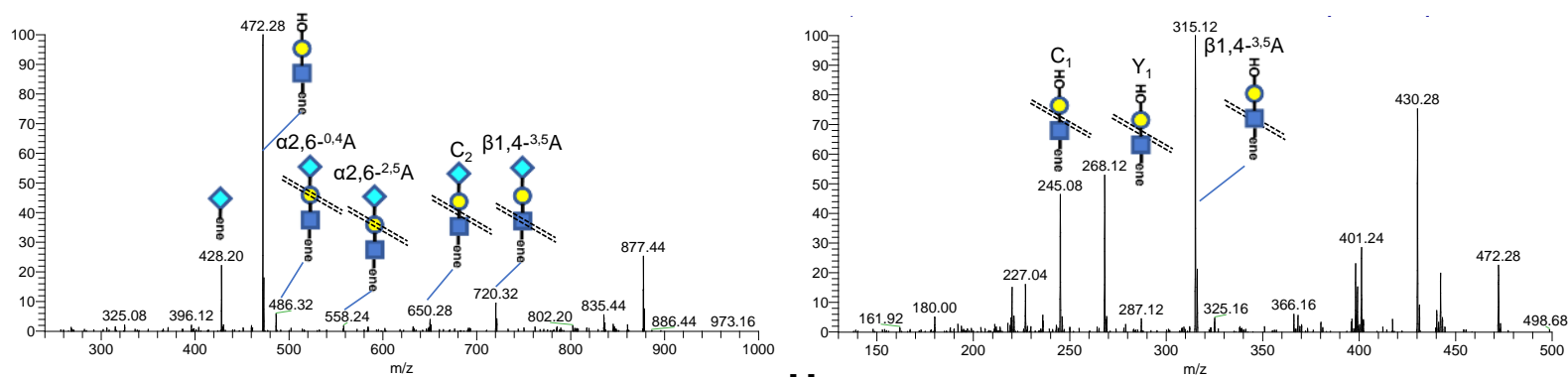

G

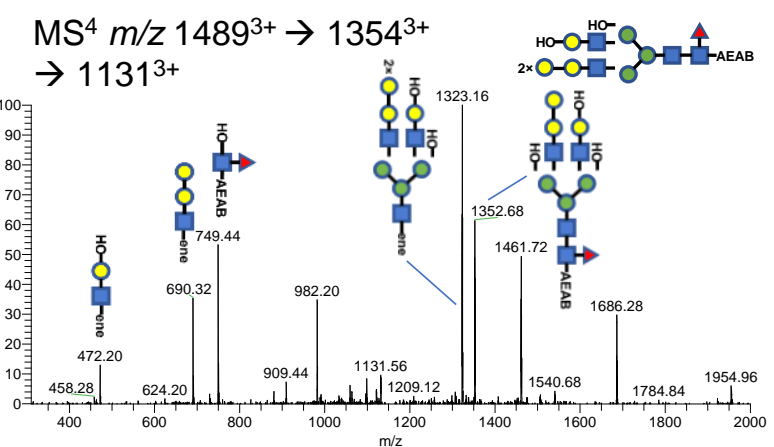

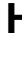

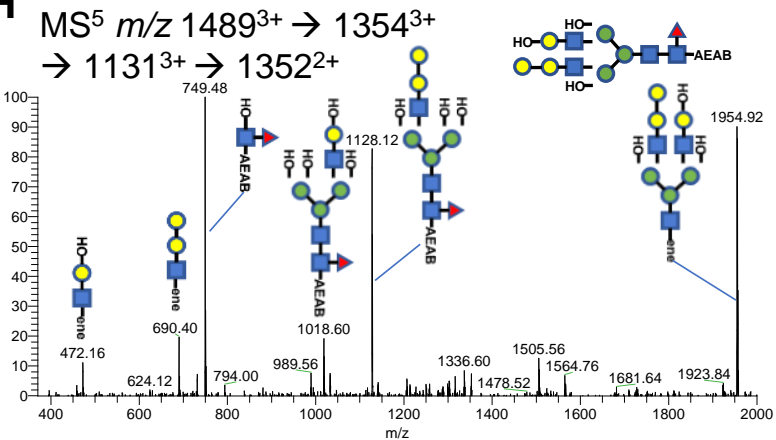

Figure S12. Structural characterization of pig kidney Fraction 80 by $\mathbf{M S}^{n}$ analysis after acetylation followed by permethylation. The original pig kidney Fraction 80 was peracetylated and then permethylated before subjected onto MS ${ }^{n}$ analysis. The order of MS and the mass-to-charge ratios $(\mathrm{m} / \mathrm{z})$ of each parent ion were indicated at the upper-left corner of each spectrum. The corresponding structure of parent fragmental ion of each analysis was showed at the upper-right corner of each spectrum. The $\mathrm{m} / \mathrm{z}$ and corresponding structure of observed fragmental ions were also showed in the figure. 


\section{Staphylococcus aureus}

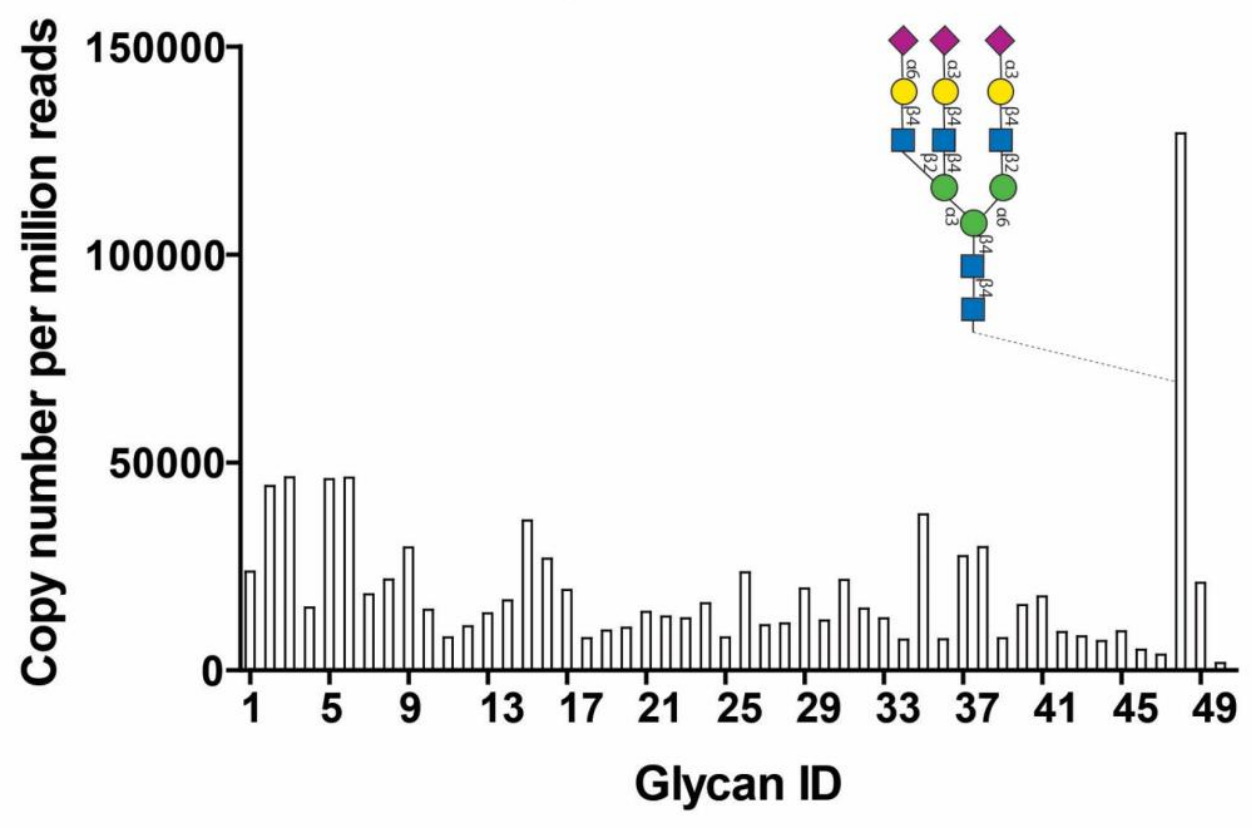

Figure S13. Glycan bindings of staphylococcus aureus by NGGM with defined glycan library. 200 $\mu \mathrm{I}$ OD 1 staphylococcus aureus were centrifugated down and re-suspended in $100 \mu \mathrm{l}$ 1XTSM buffer containing $0.05 \%$ Tween-20, $200 \mathrm{ng} / \mathrm{ml}$ salmon sperm DNA and 25 fmol defined DNA-coded glycan library. The mixture was placed on a shaker at room temperature for 1 hour. Then the bacteria were separated by centrifugation and washed for three times with $100 \mu \mathrm{l}$ 1XTSM buffer containing $0.05 \%$ Tween-20. The final bacteria were re-suspended in $100 \mu \mathrm{l}$ 1XPBS buffer. $1 \mu \mathrm{l}$ suspension containing the binding glycans which were conjugated with DNA codes was input into PCR amplification and the PCR product was decoded by NGS sequencing. The binding glycan's structure were shown in the graph. 
Table S1. Information of the library of six DNA-coded glycans. Colum A and Column B list the name and structure of six glycans; Column C shows the names of DNA codes; Column D and E show the DNA sequences of one strand and the other strand of DNA code. Note "TGTAAACGACGGCCAGT" and "GTCATAGCTGTITCCTGC" are two primer sequences for PCR amplification and indexing, the inner sequences (bolder) are coding seauences

\begin{tabular}{|c|c|c|c|c|}
\hline $\begin{array}{c}\text { Glycan } \\
\text { name }\end{array}$ & Structure & $\begin{array}{c}\text { Code } \\
\text { name }\end{array}$ & DNA sequence $\left(5^{\prime}>3^{\prime}\right)$ & DNA sequence $\left(3^{\prime} \rightarrow 5^{\prime}\right)$ \\
\hline 2'-FL & Fuca1-2Galß1-4Glc & Code1s & /5AZZideN/TGTAAAACGACGGCCAGTTGATAGGCGTCATTCTCCAGCAAGTCGTCATAGCTGTTTCCTGC & GCAGGAAACAGCTATGACGACTTGCTGGAGAATGACGCCTATCAACTGGCCGTCGTTTTACA \\
\hline 6'SL & Neu5Aa2-6Galß1-4GIc & Code2s & /5AzideN/TGTAAAACGACGGCCAGTGACAGAGACCAACGAGAGTCCAGGTCATAGCTGTTTCCTGC & GCAGGAAACAGCTATGACCTGGACTCTCGTTGGTCTCTGTCACTGGCCGTCGTTTTACA \\
\hline LNnT & 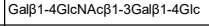 & Code3s & /5AZideN/TGTAAAACGACGGCCAGTGACCACATGTACTTTCGCAG GTCATAGCTGTTTCCTGC & GCAGGAAACAGCTATGACCTGCGAAAGTACATGTGGTCACTGGCCGTCGTTTTACA \\
\hline FBS-1 & 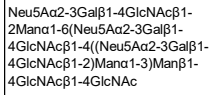 & Code4s & /5AZideN/TGTAAAACGACGGCCAGTAGGAGAAAACGCAACGTGTCATAGCTGTTTCCTGC & GCAGGAAACAGCTATGACACGTTGCGTTTTCTCCTACTGGCCGTCGTTTTACA \\
\hline Man8 & 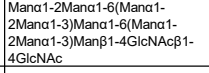 & Code5s & /5AZideN/TGTAAAACGACGGCCAGTGGATTCCAATAGTC GTCATAGCTGTTTCCTGC & GCAGGAAACAGCTATGACGACTATTGGAATCCACTGGCCGTCGTTTTACA \\
\hline EY-20 & 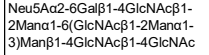 & Code6s & /5AZideN/TGTAAAACGACGGCCAGTGCGTCATTAGG GTCATAGCTGTTTCCTGC & GCAGGAAACAGCTATGACCCTAATGACGCACTGGCCGTCGTTTTACA \\
\hline
\end{tabular}




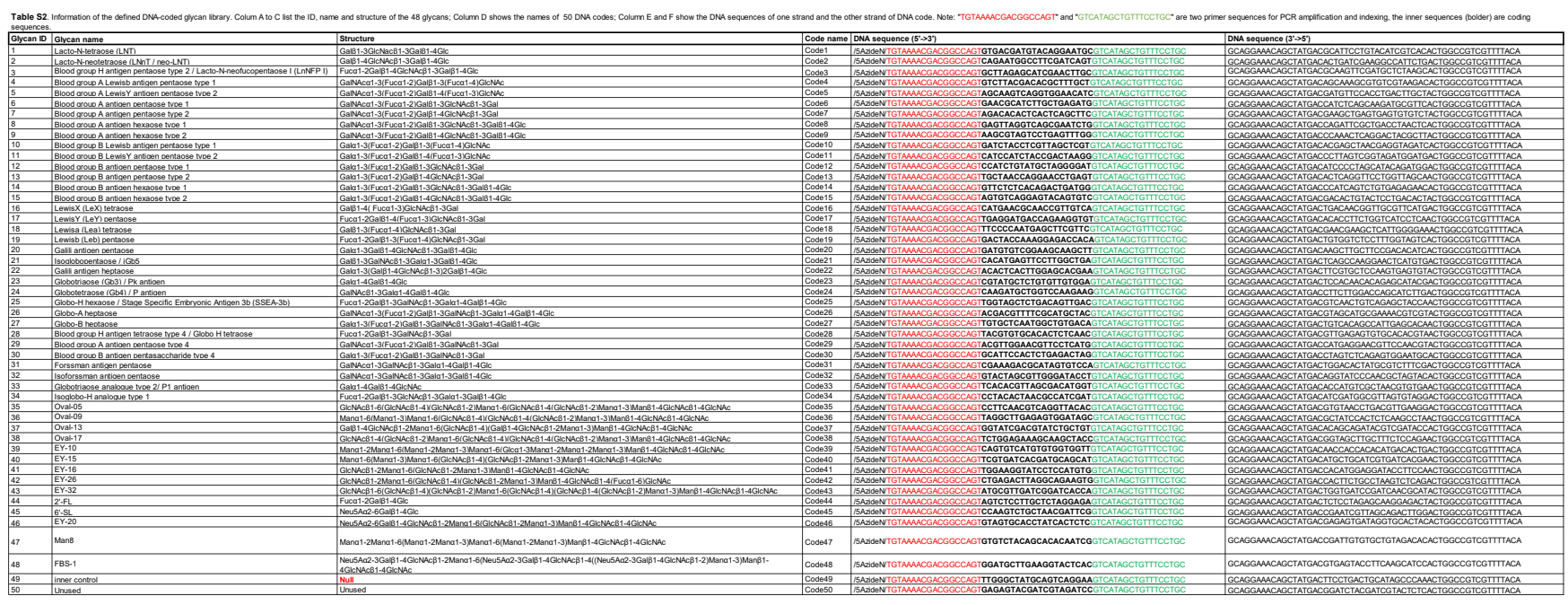


Table S3. Printed microarray raw data. Column A to C list the ID, name and structure of printed glycans; Column D is the average relative fluorescent units (RFU); Column E is the standard deviation of 4 printed spots; Column $\mathrm{F}$ is the ratio between STDEV and average RFU.

\begin{tabular}{|c|c|c|c|c|c|}
\hline \multicolumn{6}{|c|}{ ConA: $10 \mathrm{ug} / \mathrm{mL}$} \\
\hline Glycan ID & Glycan name & Structure & Average & STDEV & $\% \mathrm{CV}$ \\
\hline 1 & Lacto-N-tetraose (LNT) & Galß1-3GIcNac $\beta 1-3 G a l \beta 1-4 G \mid c$ & 335 & 59 & 18 \\
\hline 2 & Lacto-N-neotetraose (LNnT / neo-LNT) & Galß1-4GIcNAcß1-3Galß1-4GIc & 259 & 17 & 6 \\
\hline 3 & Blood group $\mathrm{H}$ antigen pentaose type 2 / Lacto-N-neofucopentaose I (LnNFP I) & Fuc $\alpha 1-2$ Gal $\beta 1-4 G I c N A c \beta 1-3 G a l \beta 1-4 G l c$ & 303 & 59 & 20 \\
\hline 4 & Blood group A Lewisb antigen pentaose type 1 & GalNAc $\alpha 1-3$ (Fuc $\alpha 1-2)$ Gal $\beta 1-3($ Fuc $\alpha 1-4)$ GIcNAc & 457 & 139 & 30 \\
\hline 5 & Blood group A LewisY antigen pentaose type 2 & GalNAc $\alpha 1-3$ (Fuc $\alpha 1-2)$ Gal $\beta 1-4($ Fuc $\alpha 1-3)$ GIcNAc & 527 & 35 & 7 \\
\hline 6 & Blood group $\mathrm{A}$ antigen pentaose type 1 & 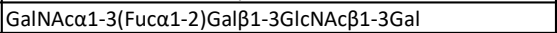 & 272 & 27 & 10 \\
\hline 7 & Blood group $\mathrm{A}$ antigen pentaose type 2 & GalNAc $\alpha 1-3$ (Fuc $\alpha 1-2)$ Gal $\beta 1-4 G I c N A c \beta 1-3 G a l$ & 291 & 124 & 42 \\
\hline 8 & Blood group $A$ antigen hexaose type 1 & GalNAc $\alpha 1-3$ (Fuc $\alpha 1-2)$ Galß1-3GIcNAc $\beta 1-3 G a l \beta 1-4 G \mid c$ & 151 & 120 & 79 \\
\hline 9 & Blood group $\mathrm{A}$ antigen hexaose type 2 & 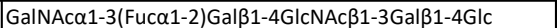 & 162 & 39 & 24 \\
\hline 10 & Blood group B Lewisb antigen pentaose type 1 & Gal $\alpha 1-3$ (Fuc $\alpha 1-2)$ Galß1-3(Fuc $\alpha 1-4)$ GIcNAc & 550 & 684 & 124 \\
\hline 11 & Blood group B LewisY antigen pentaose type 2 & Gal $\alpha 1-3$ (Fuc $\alpha 1-2)$ Gal $\beta 1-4($ Fuc $\alpha 1-3)$ GIcNAc & 203 & 19 & 9 \\
\hline 12 & Blood group B antigen pentaose type 1 & 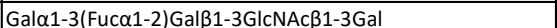 & 359 & 138 & 38 \\
\hline 13 & Blood group B antigen pentaose type 2 & Gal $\alpha 1-3$ (Fuc $\alpha 1-2)$ Gal $\beta 1-4 G I c N A c \beta 1-3 G a l$ & 179 & 81 & 45 \\
\hline 14 & Blood group B antigen hexaose type 1 & Gal $\alpha 1-3$ (Fuc $\alpha 1-2)$ Gal $\beta 1-3 G I c N A c \beta 1-3 G a l \beta 1-4 G \mid c$ & 197 & 71 & 36 \\
\hline 15 & Blood group $B$ antigen hexaose type 2 & Gal $\alpha 1-3$ (Fuc $\alpha 1-2)$ Gal $\beta 1-4 G I c N A c \beta 1-3 G a l \beta 1-4 G \mid c$ & 191 & 22 & 12 \\
\hline 16 & LewisX (LeX) tetraose & Gal $\beta 1-4$ ( Fuc $\alpha 1-3)$ GIcNAc $\beta 1-3 G a l$ & 230 & 22 & 10 \\
\hline 17 & LewisY (LeY) pentaose & Fuc $\alpha 1-2$ Gal $\beta 1-4$ (Fuc $\alpha 1-3)$ GIcNAc $\beta 1-3$ Gal & 190 & 87 & 46 \\
\hline 18 & Lewisa (Lea) tetraose & Gal $\beta 1-3($ Fuc $\alpha 1-4)$ GlcNAc $\beta 1-3 G a l$ & 90 & 45 & 50 \\
\hline 19 & Lewisb (Leb) pentaose & Fuc $\alpha 1-2$ Gal $\beta 1-3$ (Fuc $\alpha 1-4)$ GIcNAc $\beta 1-3$ Gal & 105 & 65 & 62 \\
\hline 20 & Galili antigen pentaose & Gal $\alpha 1-3 \mathrm{Gal} \beta 1-4 \mathrm{GlcNAc} \beta 1-3 \mathrm{Gal} \beta 1-4 \mathrm{Glc}$ & 82 & 28 & 34 \\
\hline 21 & Isoglobopentaose / iGb5 & Galß1-3GalNAc $\beta 1-3 G a l \alpha 1-3 G a l \beta 1-4 G \mid c$ & 231 & 29 & 13 \\
\hline 22 & Galili antigen heptaose & 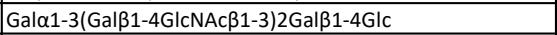 & 314 & 73 & 23 \\
\hline 23 & Globotriaose (Gb3) / Pk antigen & Gal $\alpha 1-4$ Galß1-4Glc & 294 & 109 & 37 \\
\hline 24 & Globotetraose (Gb4) / P antigen & 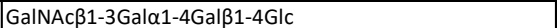 & 317 & 23 & 7 \\
\hline 25 & Globo-H hexaose / Stage Specific Embryonic Antigen 3b (SSEA-3b) & Fuc $\alpha 1-2$ Gal $\beta 1-3 G a l N A c \beta 1-3 G a l \alpha 1-4 G a l \beta 1-4 G I c$ & 202 & 53 & 26 \\
\hline 26 & Globo-A heptaose & GalNAc $\alpha 1-3$ (Fuc $\alpha 1-2)$ Galß1-3GalNAc $\beta 1-3 G a l \alpha 1-4 G a l \beta 1-4 G$ & 229 & 60 & 26 \\
\hline 27 & Globo-B heptaose & 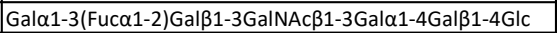 & 242 & 7 & 3 \\
\hline 28 & Blood group $\mathrm{H}$ antigen tetraose type 4 / Globo $\mathrm{H}$ tetraose & Fuc $\alpha 1-2$ Gal $\beta 1-3 G a I N A c \beta 1-3 G a l$ & 235 & 31 & 13 \\
\hline 29 & Blood group $\mathrm{A}$ antigen pentaose type 4 & GalNAc $\alpha 1-3$ (Fuc $\alpha 1-2)$ Gal $\beta 1-3 G a I N A c \beta 1-3 G a l$ & 220 & 14 & 6 \\
\hline 30 & Blood group B antigen pentasaccharide type 4 & 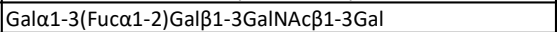 & 205 & 53 & 26 \\
\hline 31 & Forssman antigen pentaose & GalNAc $\alpha 1-3 G a l N A c \beta 1-3 G a l \alpha 1-4 G a l \beta 1-4 G \mid c$ & 146 & 41 & 28 \\
\hline 32 & Isoforssman antigen pentaose & GaINAc $\alpha 1-3 G a l N A c \beta 1-3 G a l \alpha 1-3 G a l \beta 1-4 G \mid c$ & 117 & 54 & 46 \\
\hline 33 & Globotriaose analogue type 2/ P1 antigen & Gal $\alpha 1-4$ Galß1-4GIcNAc & 233 & 68 & 29 \\
\hline 34 & Isoglobo- $\mathrm{H}$ analogue type 1 & Fuc $\alpha 1-2$ Gal $\beta 1-3 G|c N A c \beta 1-3 G a| \alpha 1-3 G a|\beta 1-4 G| c$ & 78 & 45 & 58 \\
\hline 35 & OL-05 & GIcNAc $\beta 1-6(G \mid c N A c \beta 1-4)($ GlcNAc $\beta 1-2)$ Man $\alpha 1-6(G \mid c N A c \beta 1-$ & 65366 & 77 & 0 \\
\hline 36 & OL-09 & Man $\alpha 1-6($ Man $\alpha 1-3)$ Man $\alpha 1-6(G I c N A c \beta 1-4)($ GlcNAc $\beta 1-4(G I c$ & 65309 & 111 & 0 \\
\hline 37 & OL-13 & Galß1-4GIcNAc $\beta 1-2 M a n \alpha 1-6(G \mid c N A c \beta 1-4)(G a \mid \beta 1-4 G I c N A c \beta$ & 65293 & 130 & 0 \\
\hline 38 & OL-17 & GIcNAc $\beta 1-4(G I c N A c \beta 1-2)$ Man $\alpha 1-6($ GIcNAc $\beta 1-4)(G I c N A c \beta 1-$ & 13696 & 599 & 4 \\
\hline 39 & EY-10 & Man $\alpha 1-2 M a n \alpha 1-6($ Man $\alpha 1-2 M a n \alpha 1-3)$ Man $\alpha 1-6(G \mid c \alpha 1-3 M$ & 64473 & 481 & 1 \\
\hline 40 & EY-15 & Man $\alpha 1-6($ Man $\alpha 1-3)$ Man $\alpha 1-6($ GlcNAc $\beta 1-4)($ GlcNAc $\beta 1-2 M a r$ & 64800 & 221 & 0 \\
\hline 41 & EY-16 & GIcNAc $\beta 1-2$ Man $\alpha 1-6($ GIcNAc $\beta 1-2$ Man $\alpha 1-3)$ Man $\beta 1-4 G I c N A$ & 63010 & 414 & 1 \\
\hline 42 & EY-26 & GIcNAc $\beta 1-2 M a n \alpha 1-6($ GlcNAc $\beta 1-4)(G I c N A c \beta 1-2 M a n \alpha 1-3) M$ & 65274 & 95 & 0 \\
\hline 43 & EY-32 & GIcNAc $\beta 1-6($ GIcNAc $\beta 1-4)($ GlcNAc $\beta 1-2)$ Man $\alpha 1-6($ GIcNAc $\beta 1-$ & 15755 & 1081 & 7 \\
\hline 44 & 2'-FL & Fuc $\alpha 1-2$ Galß1-4Glc & 289 & 74 & 26 \\
\hline 45 & 6'-SL & 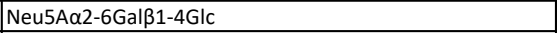 & 71 & 27 & 38 \\
\hline 46 & EY-20 & $\begin{array}{l}\text { Neu5A } \alpha 2-6 \text { Gal } \beta 1-4 G \mid c N A c \beta 1-2 \text { Man } \alpha 1-6(G \mid c N A c \beta 1- \\
2 \text { Man } \alpha 1-3) \text { Man } \beta 1-4 G \mid c N A c \beta 1-4 G I c N A c\end{array}$ & 64615 & 600 & 1 \\
\hline 47 & Man8 & 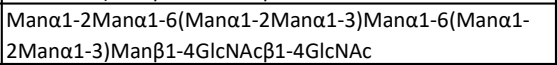 & 63857 & 672 & 1 \\
\hline 48 & FBS-1 & $\begin{array}{l}\text { Neu5A } \alpha 2-3 G a l \beta 1-4 G I c N A c \beta 1-2 \text { Man } \alpha 1-6(N e u 5 A \alpha 2-3 G a l \beta 1- \\
\text { 4GIcNAc } \beta 1-4((\text { Neu5A } \alpha 2-3 G a l \beta 1-4 G I c N A c \beta 1-2) \text { Man } \alpha 1- \\
\text { 3)Man } \beta 1-4 G I c N A c \beta 1-4 G I c N A c\end{array}$ & 385 & 58 & 15 \\
\hline 49 & Water & & 546 & 97 & 18 \\
\hline 50 & Water & & 566 & 120 & 21 \\
\hline 51 & Water & & 433 & 76 & 18 \\
\hline 52 & Biotin-BSA & & 9824 & 585 & 6 \\
\hline
\end{tabular}


Table S3. Printed microarray raw data. Column A to C list the ID, name and structure of printed glycans; Column D is the average relative fluorescent units (RFU); Column E is the standard deviation of 4 printed spots; Column F is the ratio between STDEV and average RFU.

\begin{tabular}{|c|c|c|c|c|c|}
\hline \multicolumn{6}{|c|}{ AAL: $10 \mathrm{ug} / \mathrm{mL}$} \\
\hline Glycan ID & Glycan name & Structure & Average & STDEV & $\% \mathrm{CV}$ \\
\hline 1 & Lacto-N-tetraose (LNT) & Galß1-3GlcNacß1-3Galß1-4Glc & 270 & 19 & 7 \\
\hline 2 & Lacto-N-neotetraose (LNnT / neo-LNT) & Galß1-4GIcNAcß1-3Galß1-4GIc & 60 & 39 & 65 \\
\hline 3 & Blood group $\mathrm{H}$ antigen pentaose type 2 / Lacto-N-neofucopentaose I (LnNFP I) & Fuc $\alpha 1-2$ Gal $\beta 1-4$ GIcNAc $\beta 1-3 G a l \beta 1-4 G I c$ & 63859 & 2367 & 4 \\
\hline 4 & Blood group A Lewisb antigen pentaose type 1 & GalNAc $\alpha 1-3$ (Fuc $\alpha 1-2$ )Galß1-3(Fuc $\alpha 1-4)$ GIcNAc & 63860 & 989 & 2 \\
\hline 5 & Blood group A LewisY antigen pentaose type 2 & GaINAc $\alpha 1-3$ (Fuc $\alpha 1-2$ )Galß1-4(Fuc $\alpha 1-3)$ GIcNAc & 64744 & 493 & 1 \\
\hline 6 & Blood group $\mathrm{A}$ antigen pentaose type 1 & 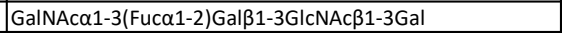 & 37 & 28 & 75 \\
\hline 7 & Blood group $\mathrm{A}$ antigen pentaose type 2 & GalNAca1-3(Fuc $\alpha 1-2)$ Galß1-4GIcNAc $\beta 1-3 G a l$ & 561 & 125 & 22 \\
\hline 8 & Blood group $A$ antigen hexaose type 1 & GalNAc $\alpha 1-3$ (Fuc $\alpha 1-2)$ Gal $\beta 1-3 G I c N A c \beta 1-3 G a l \beta 1-4 G I c$ & -20 & 12 & -59 \\
\hline 9 & Blood group $\mathrm{A}$ antigen hexaose type 2 & GalNAc $\alpha 1-3$ (Fuc $\alpha 1-2)$ Gal $\beta 1-4 G|c N A c \beta 1-3 G a l \beta 1-4 G| c$ & 1885 & 83 & 4 \\
\hline 10 & Blood group B Lewisb antigen pentaose type 1 & Gal $\alpha 1-3$ (Fuc $\alpha 1-2$ )Gal $\beta 1-3$ (Fuc $\alpha 1-4)$ GIcNAc & 65121 & 237 & 0 \\
\hline 11 & Blood group B LewisY antigen pentaose type 2 & Gal $\alpha 1-3$ (Fuc $\alpha 1-2)$ Gal $\beta 1-4($ Fuc $\alpha 1-3)$ GIcNAc & 64515 & 280 & 0 \\
\hline 12 & Blood group B antigen pentaose type 1 & Gal $\alpha 1-3$ (Fuc $\alpha 1-2$ )Gal $\beta 1-3 G \mid c N A c \beta 1-3 G$ al & 2 & 14 & 950 \\
\hline 13 & Blood group B antigen pentaose type 2 & 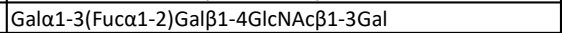 & 6079 & 624 & 10 \\
\hline 14 & Blood group B antigen hexaose type 1 & Gal $\alpha 1-3$ (Fuc $\alpha 1-2)$ Galß1-3GlcNAc $\beta 1-3 G a \mid \beta 1-4 G l c$ & 687 & 146 & 21 \\
\hline 15 & Blood group B antigen hexaose type 2 & Gal $\alpha 1-3$ (Fuc $\alpha 1-2)$ Gal/31-4GIcNAc $\beta 1-3 G a|\beta 1-4 G| c$ & 8303 & 709 & 9 \\
\hline 16 & LewisX (LeX) tetraose & Gal $\beta 1-4$ ( Fuc $\alpha 1-3)$ GIcNAc $\beta 1-3 G a l$ & 64599 & 141 & 0 \\
\hline 17 & LewisY (LeY) pentaose & 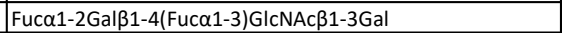 & 64656 & 865 & 1 \\
\hline 18 & Lewisa (Lea) tetraose & Galß1-3(Fuc $\alpha 1-4)$ GIcNAc $\beta 1-3 G a l$ & 65245 & 361 & 1 \\
\hline 19 & Lewisb (Leb) pentaose & 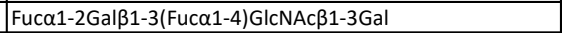 & 65126 & 250 & 0 \\
\hline 20 & Galili antigen pentaose & Gal $\alpha 1-3$ Gal $\beta 1-4 G|c N A c \beta 1-3 G a l \beta 1-4 G| c$ & -9 & 16 & -178 \\
\hline 21 & Isoglobopentaose / iGb5 & 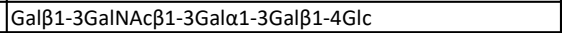 & 7 & 13 & 203 \\
\hline 22 & Galili antigen heptaose & Gal $\alpha 1-3($ Gal $\beta 1-4 G \mid c N A c \beta 1-3) 2$ Galß1-4Glc & 18 & 17 & 96 \\
\hline 23 & Globotriaose (Gb3) / Pk antigen & 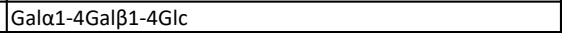 & -3 & 10 & -409 \\
\hline 24 & Globotetraose (Gb4) / $P$ antigen & 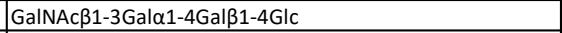 & 7 & 8 & 116 \\
\hline 25 & Globo-H hexaose / Stage Specific Embryonic Antigen 3b (SSEA-3b) & Fuc $\alpha 1-2$ Gal $\beta 1-3 G a l N A c \beta 1-3 G a l \alpha 1-4 G a l \beta 1-4 G l c$ & 65023 & 166 & 0 \\
\hline 26 & Globo-A heptaose & 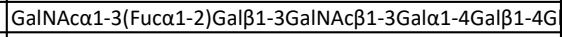 & 13 & 4 & 27 \\
\hline 27 & Globo-B heptaose & 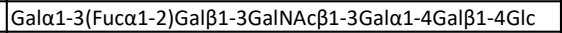 & -8 & 28 & -337 \\
\hline 28 & Blood group $\mathrm{H}$ antigen tetraose type 4 / Globo $\mathrm{H}$ tetraose & Fuc $\alpha 1-2$ Gal $\beta 1-3 G a I N A c \beta 1-3 G a l$ & 65181 & 253 & 0 \\
\hline 29 & Blood group A antigen pentaose type 4 & GalNAca1-3(Fuc $\alpha 1-2)$ Galß1-3GalNAc $\beta 1-3 G a l$ & 9 & 4 & 45 \\
\hline 30 & Blood group B antigen pentasaccharide type 4 & Gal $\alpha 1-3$ (Fuc $\alpha 1-2$ )Galß1-3GalNAc $\beta 1-3 G a l$ & 988 & 1946 & 197 \\
\hline 31 & Forssman antigen pentaose & GaINAc $\alpha 1-3$ GalNAc $\beta 1-3 G a l \alpha 1-4 G a l \beta 1-4 G I c$ & -199 & 239 & -120 \\
\hline 32 & Isoforssman antigen pentaose & GaINAc $\alpha 1-3$ GalNAc $\beta 1-3 G a l \alpha 1-3 G a l \beta 1-4 G I c$ & 11 & 8 & 74 \\
\hline 33 & Globotriaose analogue type 2/ P1 antigen & Gal 1 1-4Galß1-4GIcNAc & 1481 & 99 & 7 \\
\hline 34 & Isoglobo-H analogue type 1 & Fuc $\alpha 1-2$ Gal $\beta 1-3 G I c N A c \beta 1-3 G a l \alpha 1-3 G a l \beta 1-4 G \mid c$ & 64969 & 63 & 0 \\
\hline 35 & OL-05 & GlcNAc $\beta 1-6($ GIcNAc $\beta 1-4)(G \mid c N A c \beta 1-2)$ Man $\alpha 1-6($ GlcNAc $\beta 1-$ & -105 & 135 & -129 \\
\hline 36 & OL-09 & Man $\alpha 1-6($ Man $\alpha 1-3)$ Man $\alpha 1-6($ GlcNAc $\beta 1-4)(G I c N A c \beta 1-4$ (GIC & 10 & 5 & 57 \\
\hline 37 & $\mathrm{OL}-13$ & Gal $\beta 1-4$ GIcNAc $\beta 1-2$ Man $\alpha 1-6($ GlcNAc $\beta 1-4)(G a l \beta 1-4 G I c N A c \beta$ & 5 & 3 & 64 \\
\hline 38 & $\mathrm{OL}-17$ & GIcNAc $\beta 1-4(G I c N A c \beta 1-2)$ Man $\alpha 1-6(G I c N A c \beta 1-4)(G I c N A c \beta 1-$ & 1 & 6 & 1149 \\
\hline 39 & EY-10 & 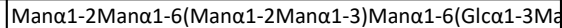 & 7 & 8 & 118 \\
\hline 40 & EY-15 & Man $\alpha 1-6($ Man $\alpha 1-3)$ Man $\alpha 1-6($ GIcNAc $\beta 1-4)(G I c N A c \beta 1-2 M a r$ & -6 & 19 & -335 \\
\hline 41 & EY-16 & GlcNAc $\beta 1-2 M a n \alpha 1-6(G l c N A c \beta 1-2 M a n \alpha 1-3)$ Man $\beta 1-4 G I c N A$ & 4 & 8 & 193 \\
\hline 42 & EY-26 & GlcNAc $\beta 1-2 M a n \alpha 1-6(G l c N A c \beta 1-4)(G l c N A c \beta 1-2 M a n \alpha 1-3) M$ & 65249 & 228 & 0 \\
\hline 43 & EY-32 & GIcNAc $\beta 1-6($ GlcNAc $\beta 1-4)(G \mid c N A c \beta 1-2) M a n \alpha 1-6(G \mid c N A c \beta 1-$ & 2269 & 134 & 6 \\
\hline 44 & 2'-FL & Fuc $\alpha 1-2$ Gal $\beta 1-4 G l c$ & 62634 & 181 & 0 \\
\hline 45 & 6'-SL & 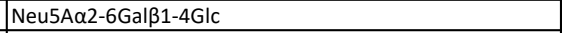 & 4 & 6 & 166 \\
\hline 46 & EY-20 & 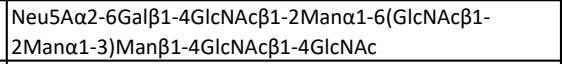 & -4 & 4 & -125 \\
\hline 47 & Man8 & 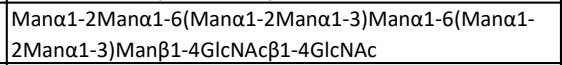 & 6 & 7 & 133 \\
\hline 48 & FBS-1 & $\begin{array}{l}\text { Neu5A } \alpha 2-3 \text { Gal } \beta 1-4 G I c N A c \beta 1-2 \text { Man } \alpha 1-6 \text { (Neu5A } \alpha 2-3 G a l \beta 1- \\
\text { 4GIcNAc } \beta 1-4((N e u 5 A \alpha 2-3 G a l \beta 1-4 G I c N A c \beta 1-2) \text { Man } \alpha 1- \\
\text { 3)Man } \beta 1-4 G I c N A c \beta 1-4 G I c N A c\end{array}$ & -36 & 19 & -54 \\
\hline 49 & Water & & 14 & 18 & 137 \\
\hline 50 & Water & & 5 & 4 & 85 \\
\hline 51 & Water & & -3 & 2 & -95 \\
\hline 52 & Biotin-BSA & & 10325 & 1218 & 12 \\
\hline
\end{tabular}


Table S3. Printed microarray raw data. Column A to C list the ID, name and structure of printed glycans; Column D is the average relative fluorescent units (RFU); Column E is the standard deviation of 4 printed spots; Column F is the ratio between STDEV and average RFU.

\begin{tabular}{|c|c|c|c|c|c|}
\hline \multicolumn{6}{|c|}{ RCA-I: $10 \mathrm{ug} / \mathrm{mL}$} \\
\hline Glycan ID & Glycan name & Structure & Average & STDEV & $\% \mathrm{CV}$ \\
\hline 1 & Lacto-N-tetraose (LNT) & Galß1-3GIcNacß1-3Galß1-4GIc & 1302 & 156 & 12 \\
\hline 2 & Lacto-N-neotetraose (LNnT / neo-LNT) & Galß1-4GIcNAcß1-3Galß1-4GIc & 65420 & 32 & 0 \\
\hline 3 & Blood group $\mathrm{H}$ antigen pentaose type 2 / Lacto-N-neofucopentaose I (LnNFP I) & Fuc $\alpha 1-2$ Gal $\beta 1-4 G I c N A c \beta 1-3 G a l \beta 1-4 G I c$ & 114 & 42 & 37 \\
\hline 4 & Blood group A Lewisb antigen pentaose type 1 & GalNAc $\alpha 1-3$ (Fuc $\alpha 1-2$ )Galß1-3(Fuc $\alpha 1-4)$ GIcNAc & 7 & 7 & 106 \\
\hline 5 & Blood group A LewisY antigen pentaose type 2 & GaINAc $\alpha 1-3$ (Fuca1-2)Galß1-4(Fuc $\alpha 1-3)$ GIcNAc & 17 & 7 & 40 \\
\hline 6 & Blood group $\mathrm{A}$ antigen pentaose type 1 & GalNAc $\alpha 1-3($ Fuc $\alpha 1-2)$ Gal $\beta 1-3 G I c N A c \beta 1-3 G a l$ & 4 & 3 & 85 \\
\hline 7 & Blood group $\mathrm{A}$ antigen pentaose type 2 & GalNAca1-3(Fuc $\alpha 1-2)$ Galß1-4GIcNAc $\beta 1-3 G a l$ & 8 & 1 & 16 \\
\hline 8 & Blood group $A$ antigen hexaose type 1 & GalNAc $\alpha 1-3$ (Fuc $\alpha 1-2)$ Gal $\beta 1-3 G I c N A c \beta 1-3 G a l \beta 1-4 G I c$ & 9 & 1 & 15 \\
\hline 9 & Blood group $\mathrm{A}$ antigen hexaose type 2 & GalNAc $\alpha 1-3$ (Fuc $\alpha 1-2)$ Gal $\beta 1-4 G I c N A c \beta 1-3 G a l \beta 1-4 G I c$ & 4 & 4 & 89 \\
\hline 10 & Blood group B Lewisb antigen pentaose type 1 & Gal $\alpha 1-3$ (Fuc $\alpha 1-2)$ Galß1-3(Fuc $\alpha 1-4) G I c N A c$ & 3 & 5 & 166 \\
\hline 11 & Blood group B LewisY antigen pentaose type 2 & Gal $\alpha 1-3$ (Fuc $\alpha 1-2$ )Galß1-4(Fuc $\alpha 1-3)$ GIcNAc & 13 & 5 & 38 \\
\hline 12 & Blood group B antigen pentaose type 1 & 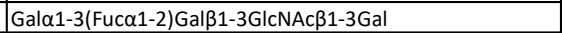 & 1 & 6 & 589 \\
\hline 13 & Blood group B antigen pentaose type 2 & Gal $\alpha 1-3$ (Fuc $\alpha 1-2$ )Gal $\beta 1-4 G I c N A c \beta 1-3 G a l$ & 4 & 6 & 149 \\
\hline 14 & Blood group B antigen hexaose type 1 & Gal $\alpha 1-3$ (Fuc $\alpha 1-2)$ Galß1-3GIcNAc $\beta 1-3 G a|\beta 1-4 G| c$ & 5 & 8 & 176 \\
\hline 15 & Blood group B antigen hexaose type 2 & Gal $\alpha 1-3($ Fuc $\alpha 1-2)$ Galß1-4GIcNAc $\beta 1-3 G a l \beta 1-4 G l c$ & 6 & 7 & 114 \\
\hline 16 & LewisX (LeX) tetraose & Galß1-4( Fuc $\alpha 1-3)$ GIcNAc $\beta 1-3 G a l$ & 3 & 8 & 334 \\
\hline 17 & LewisY (LeY) pentaose & Fuc $\alpha 1-2$ Gal $\beta 1-4$ (Fuc $\alpha 1-3)$ GlcNAc $\beta 1-3 G a l$ & 5 & 14 & 271 \\
\hline 18 & Lewisa (Lea) tetraose & Galß1-3(Fuc $\alpha 1-4)$ GlcNAc $\beta 1-3 G a l$ & 3 & 6 & 171 \\
\hline 19 & Lewisb (Leb) pentaose & Fuc $\alpha 1-2$ Gal $\beta 1-3$ (Fuc $\alpha 1-4)$ GlcNAc $\beta 1-3 G a l$ & 3 & 8 & 291 \\
\hline 20 & Galili antigen pentaose & Gal $\alpha 1-3$ Gal $\beta 1-4 G I c N A c \beta 1-3 G a l \beta 1-4 G I c$ & 967 & 171 & 18 \\
\hline 21 & Isoglobopentaose / iGb5 & 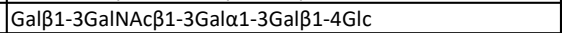 & 237 & 65 & 28 \\
\hline 22 & Galili antigen heptaose & Gal $\alpha 1-3($ Gal$\beta 1-4 G I c N A c \beta 1-3) 2 G a|\beta 1-4 G| c$ & 822 & 143 & 17 \\
\hline 23 & Globotriaose (Gb3) / Pk antigen & Gala1-4Galß1-4GIc & 227 & 34 & 15 \\
\hline 24 & Globotetraose (Gb4) / $P$ antigen & GalNAc $\beta 1-3 G a l \alpha 1-4 G a|\beta 1-4 G| c$ & -6 & 14 & -241 \\
\hline 25 & Globo-H hexaose / Stage Specific Embryonic Antigen 3b (SSEA-3b) & Fuc $\alpha 1-2$ Gal $\beta 1-3 G a l N A c \beta 1-3 G a l \alpha 1-4 G a l \beta 1-4 G \mid c$ & 8 & 4 & 47 \\
\hline 26 & Globo-A heptaose & 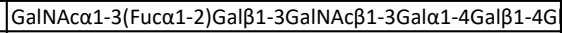 & 9 & 4 & 46 \\
\hline 27 & Globo-B heptaose & 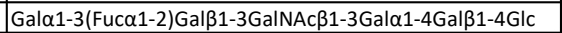 & 6 & 6 & 93 \\
\hline 28 & Blood group $\mathrm{H}$ antigen tetraose type 4 / Globo $\mathrm{H}$ tetraose & Fuc $\alpha 1-2$ Gal $\beta 1-3 G a I N A c \beta 1-3 G a l$ & -7 & 16 & -253 \\
\hline 29 & Blood group $\mathrm{A}$ antigen pentaose type 4 & 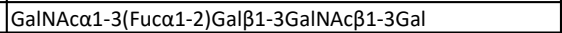 & 5 & 3 & 52 \\
\hline 30 & Blood group B antigen pentasaccharide type 4 & Gal $\alpha 1-3$ (Fuc $\alpha 1-2)$ Galß1-3GalNAc $\beta 1-3 G a l$ & 3 & 3 & 124 \\
\hline 31 & Forssman antigen pentaose & GaINAc $\alpha 1-3$ GalNAc $\beta 1-3 G a l \alpha 1-4 G a \mid \beta 1-4 G I c$ & 6 & 5 & 91 \\
\hline 32 & Isoforssman antigen pentaose & GalNAc $\alpha 1-3$ GalNAc $\beta 1-3 G a l \alpha 1-3 G a l \beta 1-4 G \mid c$ & 14 & 3 & 25 \\
\hline 33 & Globotriaose analogue type 2/ P1 antigen & Gal 1 1-4Galß1-4GIcNAc & 105 & 51 & 48 \\
\hline 34 & Isoglobo- $\mathrm{H}$ analogue type 1 & Fuc $\alpha 1-2$ Gal $\beta 1-3 G I c N A c \beta 1-3 G a l \alpha 1-3 G a l \beta 1-4 G l c$ & 73 & 18 & 25 \\
\hline 35 & OL-05 & GIcNAc $\beta 1-6($ GIcNAc $\beta 1-4)(G \mid c N A c \beta 1-2)$ Man $\alpha 1-6($ GIcNAc $\beta 1-$ & 3 & 5 & 147 \\
\hline 36 & OL-09 & Man $\alpha 1-6($ Man $\alpha 1-3)$ Man $\alpha 1-6($ GIcNAc $\beta 1-4)(G I c N A c \beta 1-4(G I C$ & 5 & 7 & 141 \\
\hline 37 & $\mathrm{OL}-13$ & 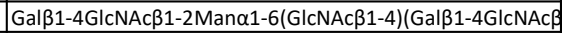 & 63110 & 714 & 1 \\
\hline 38 & OL-17 & GlcNAc $\beta 1-4($ GIcNAc $\beta 1-2)$ Man $\alpha 1-6(G I c N A c \beta 1-4)(G I c N A c \beta 1-$ & 2 & 30 & 1725 \\
\hline 39 & EY-10 & 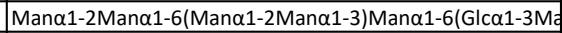 & 17 & 4 & 22 \\
\hline 40 & EY-15 & Man $\alpha 1-6($ Man $\alpha 1-3)$ Man $\alpha 1-6($ GIcNAc $\beta 1-4)(G I c N A c \beta 1-2 M a r$ & 5 & 8 & 145 \\
\hline 41 & EY-16 & GIcNAc $\beta 1-2 M a n \alpha 1-6(G I c N A c \beta 1-2 M a n \alpha 1-3)$ Man $\beta 1-4 G I c N A$ & 93 & 28 & 30 \\
\hline 42 & EY-26 & GIcNAc $\beta 1-2 M a n \alpha 1-6(G \mid c N A c \beta 1-4)(G I c N A c \beta 1-2 M a n \alpha 1-3) M$ & 4616 & 183 & 4 \\
\hline 43 & EY-32 & GlcNAc $\beta 1-6($ GIcNAc $\beta 1-4)(G \mid c N A c \beta 1-2)$ Man $\alpha 1-6($ GlcNAc $\beta 1-$ & 462 & 63 & 14 \\
\hline 44 & $2^{\prime}-\mathrm{FL}$ & Fuc $\alpha 1-2$ Gal $\beta 1-4 G l c$ & 6 & 7 & 115 \\
\hline 45 & 6'-SL & 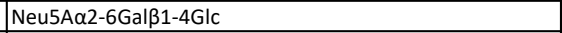 & 6274 & 591 & 9 \\
\hline 46 & EY-20 & 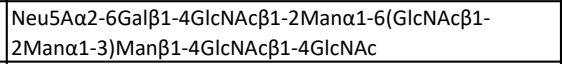 & 35842 & 5679 & 16 \\
\hline 47 & Man8 & 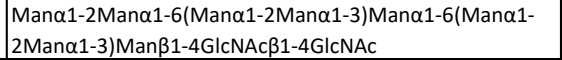 & 0 & 9 & 3686 \\
\hline 48 & FBS-1 & $\begin{array}{l}\text { Neu5A } \alpha 2-3 \text { Gal } \beta 1-4 G I c N A c \beta 1-2 M a n \alpha 1-6(\text { Neu5A } \alpha 2-3 G a l \beta 1- \\
\text { 4GIcNAc } \beta 1-4((N e u 5 A \alpha 2-3 G a l \beta 1-4 G I c N A c \beta 1-2) \text { Man } \alpha 1- \\
\text { 3)Man } \beta 1-4 G I c N A c \beta 1-4 G I c N A c\end{array}$ & 65476 & 11 & 0 \\
\hline 49 & Water & & 1 & 2 & 296 \\
\hline 50 & Water & & 3 & 2 & 62 \\
\hline 51 & Water & & -1 & 9 & -942 \\
\hline 52 & Biotin-BSA & & 4984 & 753 & 15 \\
\hline
\end{tabular}


Table S3. Printed microarray raw data. Column A to C list the ID, name and structure of printed glycans; Column D is the average relative fluorescent units (RFU); Column E is the standard deviation of 4 printed spots; Column F is the ratio between STDEV and average RFU.

\begin{tabular}{|c|c|c|c|c|c|}
\hline \multicolumn{6}{|c|}{ SNA: $10 \mathrm{ug} / \mathrm{mL}$} \\
\hline Glycan ID & Glycan name & Structure & Average & STDEV & $\% \mathrm{CV}$ \\
\hline 1 & Lacto-N-tetraose (LNT) & Galß1-3GIcNacß1-3Galß1-4GIc & 211 & 21 & 10 \\
\hline 2 & Lacto-N-neotetraose (LNnT / neo-LNT) & Galß1-4GIcNAcß1-3Galß1-4GIc & 49 & 20 & 41 \\
\hline 3 & Blood group $\mathrm{H}$ antigen pentaose type 2 / Lacto-N-neofucopentaose I (LnNFP I) & Fuc $\alpha 1-2$ Gal $\beta 1-4 G I c N A c \beta 1-3 G a l \beta 1-4 G I c$ & 25 & 17 & 67 \\
\hline 4 & Blood group A Lewisb antigen pentaose type 1 & GalNAc $\alpha 1-3$ (Fuc $\alpha 1-2$ )Galß1-3(Fuc $\alpha 1-4)$ GIcNAc & -461 & 646 & -140 \\
\hline 5 & Blood group A LewisY antigen pentaose type 2 & GaINAc $\alpha 1-3$ (Fuca1-2)Galß1-4(Fuc $\alpha 1-3)$ GIcNAc & 26 & 19 & 72 \\
\hline 6 & Blood group $\mathrm{A}$ antigen pentaose type 1 & GalNAc $\alpha 1-3($ Fuc $\alpha 1-2)$ Gal $\beta 1-3 G I c N A c \beta 1-3 G a l$ & 24 & 14 & 59 \\
\hline 7 & Blood group $\mathrm{A}$ antigen pentaose type 2 & GalNAca1-3(Fuc $\alpha 1-2)$ Galß1-4GIcNAc $\beta 1-3 G a l$ & 18 & 25 & 139 \\
\hline 8 & Blood group $A$ antigen hexaose type 1 & GalNAc $\alpha 1-3$ (Fuc $\alpha 1-2)$ Gal $\beta 1-3 G I c N A c \beta 1-3 G a l \beta 1-4 G I c$ & 0 & 32 & -12705 \\
\hline 9 & Blood group $\mathrm{A}$ antigen hexaose type 2 & GalNAc $\alpha 1-3$ (Fuc $\alpha 1-2)$ Gal $\beta 1-4 G I c N A c \beta 1-3 G a l \beta 1-4 G I c$ & 29 & 48 & 170 \\
\hline 10 & Blood group B Lewisb antigen pentaose type 1 & Gal $\alpha 1-3$ (Fuc $\alpha 1-2)$ Galß1-3(Fuc $\alpha 1-4) G I c N A c$ & 7 & 7 & 93 \\
\hline 11 & Blood group B LewisY antigen pentaose type 2 & Gal $\alpha 1-3$ (Fuc $\alpha 1-2$ )Galß1-4(Fuc $\alpha 1-3)$ GIcNAc & -10 & 31 & -327 \\
\hline 12 & Blood group B antigen pentaose type 1 & Gal $\alpha 1-3$ (Fuc $\alpha 1-2$ )Gal $\beta 1-3 G \mid c N A c \beta 1-3 G a l$ & -11 & 30 & -266 \\
\hline 13 & Blood group B antigen pentaose type 2 & Gal $\alpha 1-3$ (Fuc $\alpha 1-2$ )Galß1-4GIcNAc $\beta 1-3 G a l$ & 9 & 1 & 16 \\
\hline 14 & Blood group B antigen hexaose type 1 & Gal $\alpha 1-3$ (Fuc $\alpha 1-2)$ Galß1-3GIcNAc $\beta 1-3 G a|\beta 1-4 G| c$ & 4 & 3 & 82 \\
\hline 15 & Blood group B antigen hexaose type 2 & Gal $\alpha 1-3($ Fuc $\alpha 1-2)$ Galß1-4GIcNAc $\beta 1-3 G a l \beta 1-4 G l c$ & 11 & 4 & 37 \\
\hline 16 & LewisX (LeX) tetraose & Galß1-4( Fuc $\alpha 1-3)$ GIcNAc $\beta 1-3 G a l$ & 9 & 3 & 33 \\
\hline 17 & LewisY (LeY) pentaose & Fuc $\alpha 1-2$ Gal $\beta 1-4$ (Fuc $\alpha 1-3)$ GlcNAc $\beta 1-3 G a l$ & 4 & 5 & 156 \\
\hline 18 & Lewisa (Lea) tetraose & Gal $\beta 1-3$ (Fuc $\alpha 1-4)$ GIcNAc $\beta 1-3 G a l$ & 5 & 7 & 157 \\
\hline 19 & Lewisb (Leb) pentaose & Fuc $\alpha 1-2$ Gal $\beta 1-3$ (Fuc $\alpha 1-4)$ GlcNAc $\beta 1-3 G a l$ & 6 & 2 & 30 \\
\hline 20 & Galili antigen pentaose & Gal $\alpha 1-3$ Gal $\beta 1-4 G I c N A c \beta 1-3 G a l \beta 1-4 G I c$ & 4 & 5 & 114 \\
\hline 21 & Isoglobopentaose / iGb5 & 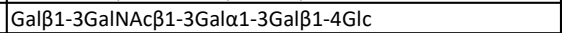 & 6 & 3 & 52 \\
\hline 22 & Galili antigen heptaose & Gal $\alpha 1-3($ Gal$\beta 1-4 G I c N A c \beta 1-3) 2 G a|\beta 1-4 G| c$ & 2 & 5 & 206 \\
\hline 23 & Globotriaose (Gb3) / Pk antigen & Gala1-4Galß1-4GIc & 26 & 27 & 106 \\
\hline 24 & Globotetraose (Gb4) / $P$ antigen & GalNAc $\beta 1-3 G a l \alpha 1-4 G a|\beta 1-4 G| c$ & 23 & 15 & 64 \\
\hline 25 & Globo-H hexaose / Stage Specific Embryonic Antigen 3b (SSEA-3b) & Fuc $\alpha 1-2$ Gal $\beta 1-3 G a l N A c \beta 1-3 G a l \alpha 1-4 G a l \beta 1-4 G \mid c$ & 693 & 45 & 7 \\
\hline 26 & Globo-A heptaose & 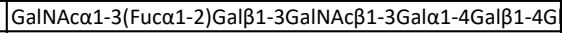 & 4 & 6 & 175 \\
\hline 27 & Globo-B heptaose & 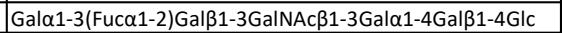 & 4 & 6 & 140 \\
\hline 28 & Blood group $\mathrm{H}$ antigen tetraose type 4 / Globo $\mathrm{H}$ tetraose & Fuc $\alpha 1-2$ Gal $\beta 1-3 G a I N A c \beta 1-3 G a l$ & 834 & 128 & 15 \\
\hline 29 & Blood group $\mathrm{A}$ antigen pentaose type 4 & 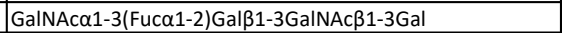 & 2 & 6 & 294 \\
\hline 30 & Blood group B antigen pentasaccharide type 4 & Gal $\alpha 1-3$ (Fuc $\alpha 1-2)$ Galß1-3GalNAc $\beta 1-3 G a l$ & 7 & 5 & 74 \\
\hline 31 & Forssman antigen pentaose & GaINAc $\alpha 1-3$ GalNAc $\beta 1-3 G a l \alpha 1-4 G a \mid \beta 1-4 G I c$ & 10 & 5 & 50 \\
\hline 32 & Isoforssman antigen pentaose & GaINAc $\alpha 1-3 G a I N A c \beta 1-3 G a l \alpha 1-3 G a l \beta 1-4 G \mid c$ & 5 & 7 & 138 \\
\hline 33 & Globotriaose analogue type 2/ P1 antigen & Gal 1 1-4Galß1-4GIcNAc & 3 & 7 & 221 \\
\hline 34 & Isoglobo- $\mathrm{H}$ analogue type 1 & Fuc $\alpha 1-2$ Gal $\beta 1-3 G I c N A c \beta 1-3 G a l \alpha 1-3 G a l \beta 1-4 G l c$ & 7 & 7 & 99 \\
\hline 35 & OL-05 & GIcNAc $\beta 1-6($ GIcNAc $\beta 1-4)(G \mid c N A c \beta 1-2)$ Man $\alpha 1-6($ GIcNAc $\beta 1-$ & 4 & 4 & 96 \\
\hline 36 & OL-09 & Man $\alpha 1-6($ Man $\alpha 1-3)$ Man $\alpha 1-6($ GIcNAc $\beta 1-4)($ GlcNAc $\beta 1-4(G I C$ & 2 & 6 & 314 \\
\hline 37 & $\mathrm{OL}-13$ & 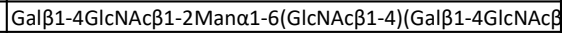 & 5 & 5 & 108 \\
\hline 38 & $\mathrm{OL}-17$ & GlcNAc $\beta 1-4($ GIcNAc $\beta 1-2)$ Man $\alpha 1-6(G I c N A c \beta 1-4)(G I c N A c \beta 1-$ & 8 & 7 & 92 \\
\hline 39 & EY-10 & 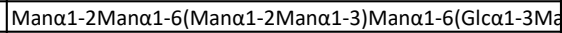 & 76 & 43 & 56 \\
\hline 40 & EY-15 & Man $\alpha 1-6($ Man $\alpha 1-3)$ Man $\alpha 1-6($ GIcNAc $\beta 1-4)(G I c N A c \beta 1-2 M a r$ & 5 & 2 & 43 \\
\hline 41 & EY-16 & GIcNAc $\beta 1-2 M a n \alpha 1-6(G I c N A c \beta 1-2 M a n \alpha 1-3)$ Man $\beta 1-4 G I c N A$ & 2 & 6 & 318 \\
\hline 42 & EY-26 & GlcNAc $\beta 1-2 M a n \alpha 1-6(G l c N A c \beta 1-4)(G l c N A c \beta 1-2 M a n \alpha 1-3) M$ & 18 & 8 & 48 \\
\hline 43 & EY-32 & GlcNAc $\beta 1-6($ GIcNAc $\beta 1-4)(G \mid c N A c \beta 1-2)$ Man $\alpha 1-6($ GlcNAc $\beta 1-$ & 3 & 4 & 119 \\
\hline 44 & $2^{\prime}-\mathrm{FL}$ & Fuc $\alpha 1-2$ Gal $\beta 1-4 G l c$ & 9 & 14 & 152 \\
\hline 45 & 6'-SL & 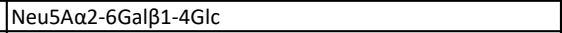 & 133 & 96 & 72 \\
\hline 46 & EY-20 & 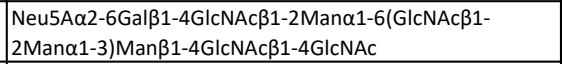 & 44829 & 947 & 2 \\
\hline 47 & Man8 & 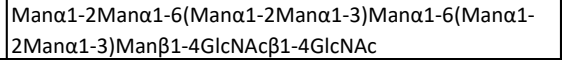 & 2 & 5 & 248 \\
\hline 48 & FBS-1 & $\begin{array}{l}\text { Neu5A } \alpha 2-3 \text { Gal } \beta 1-4 G I c N A c \beta 1-2 M a n \alpha 1-6(\text { Neu5A } \alpha 2-3 G a l \beta 1- \\
\text { 4GIcNAc } \beta 1-4((N e u 5 A \alpha 2-3 G a l \beta 1-4 G I c N A c \beta 1-2) \text { Man } \alpha 1- \\
\text { 3)Man } \beta 1-4 G I c N A c \beta 1-4 G I c N A c\end{array}$ & 24131 & 5490 & 23 \\
\hline 49 & Water & & 4 & 6 & 157 \\
\hline 50 & Water & & 1 & 7 & 894 \\
\hline 51 & Water & & 2 & 5 & 351 \\
\hline 52 & Biotin-BSA & & 10257 & 1432 & 14 \\
\hline
\end{tabular}


Table S3. Printed microarray raw data. Column A to C list the ID, name and structure of printed glycans; Column D is the average relative fluorescent units (RFU); Column E is the standard deviation of 4 printed spots; Column $\mathrm{F}$ is the ratio between STDEV and average RFU.

\begin{tabular}{|c|c|c|c|c|c|}
\hline \multicolumn{6}{|c|}{ MAL-I: $10 \mathrm{ug} / \mathrm{mL}$} \\
\hline Glycan ID & Glycan name & Structure & Average & STDEV & $\% \mathrm{CV}$ \\
\hline 1 & Lacto-N-tetraose (LNT) & Galß1-3GIcNacß1-3Galß1-4GIc & 159 & 48 & 30 \\
\hline 2 & Lacto-N-neotetraose (LNnT / neo-LNT) & Galß1-4GIcNAcß1-3Galß1-4GIc & 989 & 308 & 31 \\
\hline 3 & Blood group $\mathrm{H}$ antigen pentaose type 2 / Lacto-N-neofucopentaose I (LnNFP I) & Fuc $\alpha 1-2$ Gal $\beta 1-4$ GIcNAc $\beta 1-3 G a l \beta 1-4 G I c$ & 5 & 6 & 134 \\
\hline 4 & Blood group A Lewisb antigen pentaose type 1 & GalNAc $\alpha 1-3$ (Fuc $\alpha 1-2$ )Galß1-3(Fuc $\alpha 1-4)$ GIcNAc & 10 & 8 & 80 \\
\hline 5 & Blood group A LewisY antigen pentaose type 2 & GaINAc $\alpha 1-3$ (Fuca1-2)Galß1-4(Fuc $\alpha 1-3)$ GIcNAc & 16 & 12 & 74 \\
\hline 6 & Blood group $\mathrm{A}$ antigen pentaose type 1 & GalNAc $\alpha 1-3($ Fuc $\alpha 1-2)$ Gal $\beta 1-3 G I c N A c \beta 1-3 G a l$ & 7 & 2 & 27 \\
\hline 7 & Blood group $\mathrm{A}$ antigen pentaose type 2 & GalNAca1-3(Fuc $\alpha 1-2)$ Galß1-4GIcNAc $\beta 1-3 G a l$ & 25 & 42 & 171 \\
\hline 8 & Blood group $A$ antigen hexaose type 1 & GalNAc $\alpha 1-3$ (Fuc $\alpha 1-2)$ Gal $\beta 1-3 G I c N A c \beta 1-3 G a l \beta 1-4 G I c$ & 4 & 4 & 127 \\
\hline 9 & Blood group $\mathrm{A}$ antigen hexaose type 2 & GalNAc $\alpha 1-3$ (Fuc $\alpha 1-2)$ Gal $\beta 1-4 G I c N A c \beta 1-3 G a l \beta 1-4 G I c$ & 5 & 3 & 52 \\
\hline 10 & Blood group B Lewisb antigen pentaose type 1 & Gal $\alpha 1-3$ (Fuc $\alpha 1-2)$ Galß1-3(Fuc $\alpha 1-4) G I c N A c$ & 4 & 2 & 46 \\
\hline 11 & Blood group B LewisY antigen pentaose type 2 & Gal $\alpha 1-3$ (Fuc $\alpha 1-2$ )Galß1-4(Fuc $\alpha 1-3)$ GIcNAc & 7 & 3 & 46 \\
\hline 12 & Blood group B antigen pentaose type 1 & 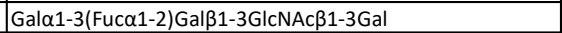 & 1 & 2 & 245 \\
\hline 13 & Blood group B antigen pentaose type 2 & Gal $\alpha 1-3$ (Fuc $\alpha 1-2$ )Gal $\beta 1-4 G I c N A c \beta 1-3 G a l$ & 3 & 3 & 99 \\
\hline 14 & Blood group B antigen hexaose type 1 & Gal $\alpha 1-3$ (Fuc $\alpha 1-2)$ Galß1-3GIcNAc $\beta 1-3 G a|\beta 1-4 G| c$ & 4 & 4 & 100 \\
\hline 15 & Blood group B antigen hexaose type 2 & Gal $\alpha 1-3$ (Fuc $\alpha 1-2)$ Gal $\beta 1-4 G|c N A c \beta 1-3 G a l \beta 1-4 G| c$ & 4 & 6 & 149 \\
\hline 16 & LewisX (LeX) tetraose & Galß1-4( Fuc $\alpha 1-3)$ GIcNAc $\beta 1-3 G a l$ & 4 & 6 & 166 \\
\hline 17 & LewisY (LeY) pentaose & Fuc $\alpha 1-2$ Gal $\beta 1-4$ (Fuc $\alpha 1-3)$ GlcNAc $\beta 1-3 G a l$ & 3 & 3 & 109 \\
\hline 18 & Lewisa (Lea) tetraose & Galß1-3(Fuc $\alpha 1-4)$ GlcNAc $\beta 1-3 G a l$ & 4 & 6 & 143 \\
\hline 19 & Lewisb (Leb) pentaose & Fuc $\alpha 1-2$ Gal $\beta 1-3$ (Fuc $\alpha 1-4)$ GlcNAc $\beta 1-3 G a l$ & 4 & 5 & 156 \\
\hline 20 & Galili antigen pentaose & Gal $\alpha 1-3$ Gal $\beta 1-4 G I c N A c \beta 1-3 G a l \beta 1-4 G I c$ & 7 & 7 & 106 \\
\hline 21 & Isoglobopentaose / iGb5 & 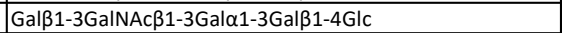 & 6 & 4 & 81 \\
\hline 22 & Galili antigen heptaose & Gal $\alpha 1-3($ Gal$\beta 1-4 G I c N A c \beta 1-3) 2 G a|\beta 1-4 G| c$ & 5 & 4 & 68 \\
\hline 23 & Globotriaose (Gb3) / Pk antigen & Gala1-4Galß1-4GIc & 9 & 5 & 58 \\
\hline 24 & Globotetraose (Gb4) / $P$ antigen & GalNAc $\beta 1-3 G a l \alpha 1-4 G a|\beta 1-4 G| c$ & 1 & 4 & 309 \\
\hline 25 & Globo-H hexaose / Stage Specific Embryonic Antigen 3b (SSEA-3b) & Fuc $\alpha 1-2$ Gal $\beta 1-3 G a l N A c \beta 1-3 G a l \alpha 1-4 G a l \beta 1-4 G \mid c$ & 3 & 3 & 99 \\
\hline 26 & Globo-A heptaose & 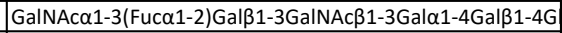 & 6 & 4 & 61 \\
\hline 27 & Globo-B heptaose & 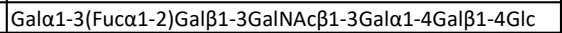 & 3 & 5 & 210 \\
\hline 28 & Blood group $\mathrm{H}$ antigen tetraose type 4 / Globo $\mathrm{H}$ tetraose & Fuc $\alpha 1-2$ Gal $\beta 1-3 G a I N A c \beta 1-3 G a l$ & 9 & 2 & 20 \\
\hline 29 & Blood group $\mathrm{A}$ antigen pentaose type 4 & 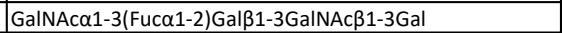 & 7 & 8 & 110 \\
\hline 30 & Blood group B antigen pentasaccharide type 4 & Gal $\alpha 1-3$ (Fuc $\alpha 1-2)$ Galß1-3GalNAc $\beta 1-3 G a l$ & 2 & 5 & 239 \\
\hline 31 & Forssman antigen pentaose & GaINAc $\alpha 1-3$ GalNAc $\beta 1-3 G a l \alpha 1-4 G a \mid \beta 1-4 G I c$ & 6 & 2 & 33 \\
\hline 32 & Isoforssman antigen pentaose & GalNAc $\alpha 1-3$ GalNAc $\beta 1-3 G a l \alpha 1-3 G a l \beta 1-4 G \mid c$ & 10 & 5 & 53 \\
\hline 33 & Globotriaose analogue type 2/ P1 antigen & Gal 1 1-4Galß1-4GIcNAc & 8 & 4 & 52 \\
\hline 34 & Isoglobo- $\mathrm{H}$ analogue type 1 & Fuc $\alpha 1-2$ Gal $\beta 1-3 G I c N A c \beta 1-3 G a l \alpha 1-3 G a l \beta 1-4 G l c$ & 4 & 10 & 288 \\
\hline 35 & OL-05 & GIcNAc $\beta 1-6($ GIcNAc $\beta 1-4)(G \mid c N A c \beta 1-2)$ Man $\alpha 1-6($ GIcNAc $\beta 1-$ & 3 & 5 & 180 \\
\hline 36 & OL-09 & Man $\alpha 1-6($ Man $\alpha 1-3)$ Man $\alpha 1-6($ GIcNAc $\beta 1-4)(G I c N A c \beta 1-4(G I C$ & 5 & 5 & 105 \\
\hline 37 & $\mathrm{OL}-13$ & 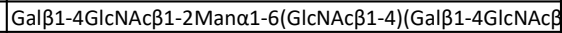 & 22 & 9 & 41 \\
\hline 38 & $\mathrm{OL}-17$ & GlcNAc $\beta 1-4(G I c N A c \beta 1-2)$ Man $\alpha 1-6(G I c N A c \beta 1-4)(G I c N A c \beta 1-$ & 5 & 4 & 90 \\
\hline 39 & EY-10 & 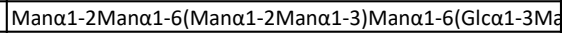 & 1 & 2 & 296 \\
\hline 40 & EY-15 & Man $\alpha 1-6($ Man $\alpha 1-3)$ Man $\alpha 1-6($ GIcNAc $\beta 1-4)(G I c N A c \beta 1-2 M a r$ & 6 & 2 & 36 \\
\hline 41 & EY-16 & GIcNAc $\beta 1-2 M a n \alpha 1-6(G I c N A c \beta 1-2 M a n \alpha 1-3)$ Man $\beta 1-4 G I c N A$ & 6 & 5 & 92 \\
\hline 42 & EY-26 & GlcNAc $\beta 1-2 M a n \alpha 1-6(G l c N A c \beta 1-4)(G l c N A c \beta 1-2 M a n \alpha 1-3) M$ & 1 & 1 & 115 \\
\hline 43 & EY-32 & GlcNAc $\beta 1-6($ GIcNAc $\beta 1-4)(G \mid c N A c \beta 1-2)$ Man $\alpha 1-6($ GlcNAc $\beta 1-$ & 1 & 2 & 416 \\
\hline 44 & $2^{\prime}-\mathrm{FL}$ & Fuc $\alpha 1-2$ Gal $\beta 1-4 G l c$ & 6 & 5 & 77 \\
\hline 45 & 6'-SL & 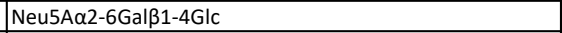 & 2 & 4 & 216 \\
\hline 46 & EY-20 & 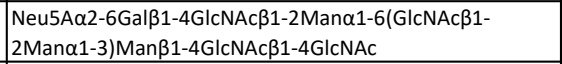 & 12 & 18 & 160 \\
\hline 47 & Man8 & 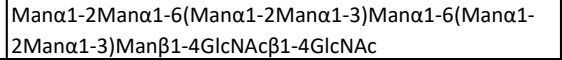 & 1 & 4 & 288 \\
\hline 48 & FBS-1 & $\begin{array}{l}\text { Neu5A } \alpha 2-3 \text { Gal } \beta 1-4 G I c N A c \beta 1-2 M a n \alpha 1-6(\text { Neu5A } \alpha 2-3 G a l \beta 1- \\
\text { 4GIcNAc } \beta 1-4((N e u 5 A \alpha 2-3 G a l \beta 1-4 G I c N A c \beta 1-2) \text { Man } \alpha 1- \\
\text { 3)Man } \beta 1-4 G I c N A c \beta 1-4 G I c N A c\end{array}$ & 65496 & 2 & 0 \\
\hline 49 & Water & & 3 & 3 & 92 \\
\hline 50 & Water & & 3 & 11 & 325 \\
\hline 51 & Water & & 2 & 3 & 128 \\
\hline 52 & Biotin-BSA & & 8063 & 844 & 10 \\
\hline
\end{tabular}


Table S3. Printed microarray raw data. Column A to C list the ID, name and structure of printed glycans; Column D is the average relative fluorescent units (RFU); Column E is the standard deviation of 4 printed spots; Column F is the ratio between STDEV and average RFU.

\begin{tabular}{|c|c|c|c|c|c|}
\hline \multicolumn{6}{|c|}{ HPA: $10 \mathrm{ug} / \mathrm{mL}$} \\
\hline Glycan ID & Glycan name & Structure & Average & STDEV & $\% \mathrm{CV}$ \\
\hline 1 & Lacto-N-tetraose (LNT) & Galß1-3GIcNacß1-3Galß1-4GIc & 162 & 58 & 36 \\
\hline 2 & Lacto-N-neotetraose (LNnT / neo-LNT) & Galß1-4GIcNAc $\beta 1-3 G a l \beta 1-4 G \mid c$ & 49 & 17 & 34 \\
\hline 3 & Blood group $\mathrm{H}$ antigen pentaose type 2 / Lacto-N-neofucopentaose I (LnNFP I) & Fuc $\alpha 1-2$ Gal $\beta 1-4 G I c N A c \beta 1-3 G a l \beta 1-4 G I c$ & 55 & 6 & 11 \\
\hline 4 & Blood group A Lewisb antigen pentaose type 1 & GalNAc $\alpha 1-3$ (Fuc $\alpha 1-2$ )Galß1-3(Fuc $\alpha 1-4)$ GIcNAc & 64351 & 1009 & 2 \\
\hline 5 & Blood group A LewisY antigen pentaose type 2 & GaINAc $\alpha 1-3$ (Fuca1-2)Galß1-4(Fuc $\alpha 1-3)$ GIcNAc & 63950 & 915 & 1 \\
\hline 6 & Blood group $\mathrm{A}$ antigen pentaose type 1 & GalNAc $\alpha 1-3($ Fuc $\alpha 1-2)$ Gal $\beta 1-3 G I c N A c \beta 1-3 G a l$ & 64406 & 1181 & 2 \\
\hline 7 & Blood group $\mathrm{A}$ antigen pentaose type 2 & GalNAca1-3(Fuc $\alpha 1-2)$ Galß1-4GIcNAc $\beta 1-3 G a l$ & 64632 & 668 & 1 \\
\hline 8 & Blood group $A$ antigen hexaose type 1 & GalNAc $\alpha 1-3$ (Fuc $\alpha 1-2)$ Gal $\beta 1-3 G I c N A c \beta 1-3 G a l \beta 1-4 G I c$ & 64929 & 344 & 1 \\
\hline 9 & Blood group $\mathrm{A}$ antigen hexaose type 2 & GalNAc $\alpha 1-3$ (Fuc $\alpha 1-2)$ Gal $\beta 1-4 G I c N A c \beta 1-3 G a l \beta 1-4 G I c$ & 65396 & 157 & 0 \\
\hline 10 & Blood group B Lewisb antigen pentaose type 1 & Gal $\alpha 1-3$ (Fuc $\alpha 1-2)$ Galß1-3(Fuc $\alpha 1-4) G I c N A c$ & -3 & 2 & -69 \\
\hline 11 & Blood group B LewisY antigen pentaose type 2 & Gal $\alpha 1-3$ (Fuc $\alpha 1-2$ )Galß1-4(Fuc $\alpha 1-3)$ GIcNAc & 6 & 9 & 159 \\
\hline 12 & Blood group B antigen pentaose type 1 & 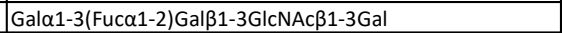 & 7 & 7 & 101 \\
\hline 13 & Blood group B antigen pentaose type 2 & Gal $\alpha 1-3$ (Fuc $\alpha 1-2$ )Gal $\beta 1-4 G I c N A c \beta 1-3 G a l$ & 6 & 6 & 99 \\
\hline 14 & Blood group B antigen hexaose type 1 & Gal $\alpha 1-3$ (Fuc $\alpha 1-2)$ Galß1-3GIcNAc $\beta 1-3 G a|\beta 1-4 G| c$ & 10 & 5 & 52 \\
\hline 15 & Blood group B antigen hexaose type 2 & Gal $\alpha 1-3$ (Fuc $\alpha 1-2)$ Gal $\beta 1-4 G|c N A c \beta 1-3 G a l \beta 1-4 G| c$ & 7 & 11 & 171 \\
\hline 16 & LewisX (LeX) tetraose & Galß1-4( Fuc $\alpha 1-3)$ GIcNAc $\beta 1-3 G a l$ & 10 & 3 & 29 \\
\hline 17 & LewisY (LeY) pentaose & Fuc $\alpha 1-2$ Gal $\beta 1-4($ Fuc $\alpha 1-3)$ GlcNAc $\beta 1-3 G a l$ & 11 & 10 & 98 \\
\hline 18 & Lewisa (Lea) tetraose & Gal $\beta 1-3$ (Fuc $\alpha 1-4)$ GIcNAc $\beta 1-3 G a l$ & 12 & 14 & 112 \\
\hline 19 & Lewisb (Leb) pentaose & Fuc $\alpha 1-2$ Gal $\beta 1-3$ (Fuc $\alpha 1-4)$ GlcNAc $\beta 1-3 G a l$ & 6 & 3 & 49 \\
\hline 20 & Galili antigen pentaose & Gal $\alpha 1-3$ Gal $\beta 1-4 G I c N A c \beta 1-3 G a l \beta 1-4 G I c$ & 14 & 6 & 44 \\
\hline 21 & Isoglobopentaose / iGb5 & 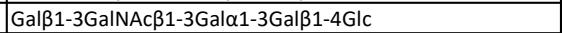 & 21 & 7 & 34 \\
\hline 22 & Galili antigen heptaose & Gal $\alpha 1-3($ Gal$\beta 1-4 G I c N A c \beta 1-3) 2 G a|\beta 1-4 G| c$ & 25 & 8 & 30 \\
\hline 23 & Globotriaose (Gb3) / Pk antigen & Gala1-4Galß1-4GIc & 5 & 7 & 130 \\
\hline 24 & Globotetraose (Gb4) / $P$ antigen & 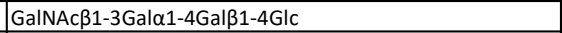 & -3 & 33 & -1115 \\
\hline 25 & Globo-H hexaose / Stage Specific Embryonic Antigen 3b (SSEA-3b) & Fuc $\alpha 1-2$ Gal $\beta 1-3 G a l N A c \beta 1-3 G a l \alpha 1-4 G a l \beta 1-4 G \mid c$ & 30 & 7 & 23 \\
\hline 26 & Globo-A heptaose & 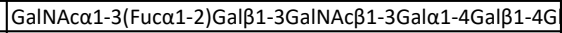 & 63551 & 323 & 1 \\
\hline 27 & Globo-B heptaose & 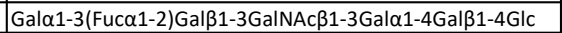 & 3 & 15 & 610 \\
\hline 28 & Blood group $\mathrm{H}$ antigen tetraose type 4 / Globo $\mathrm{H}$ tetraose & Fuc $\alpha 1-2$ Gal $\beta 1-3 G a I N A c \beta 1-3 G a l$ & -24 & 45 & -190 \\
\hline 29 & Blood group $\mathrm{A}$ antigen pentaose type 4 & 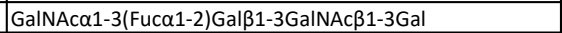 & 65187 & 298 & 0 \\
\hline 30 & Blood group B antigen pentasaccharide type 4 & Gal $\alpha 1-3$ (Fuc $\alpha 1-2)$ Galß1-3GalNAc $\beta 1-3 G a l$ & 1 & 11 & 870 \\
\hline 31 & Forssman antigen pentaose & GaINAc $\alpha 1-3$ GalNAc $\beta 1-3 G a l \alpha 1-4 G a \mid \beta 1-4 G I c$ & 64096 & 331 & 1 \\
\hline 32 & Isoforssman antigen pentaose & GalNAc $\alpha 1-3$ GalNAc $\beta 1-3 G a l \alpha 1-3 G a l \beta 1-4 G \mid c$ & 64523 & 175 & 0 \\
\hline 33 & Globotriaose analogue type 2/ P1 antigen & Gal 1 1-4Galß1-4GIcNAc & -2 & 2 & -122 \\
\hline 34 & Isoglobo- $\mathrm{H}$ analogue type 1 & Fuc $\alpha 1-2$ Gal $\beta 1-3 G I c N A c \beta 1-3 G a l \alpha 1-3 G a l \beta 1-4 G l c$ & 50 & 105 & 211 \\
\hline 35 & OL-05 & GIcNAc $\beta 1-6($ GIcNAc $\beta 1-4)(G \mid c N A c \beta 1-2)$ Man $\alpha 1-6($ GIcNAc $\beta 1-$ & -4 & 3 & -73 \\
\hline 36 & OL-09 & Man $\alpha 1-6($ Man $\alpha 1-3)$ Man $\alpha 1-6($ GIcNAc $\beta 1-4)(G I c N A c \beta 1-4(G I C$ & 24146 & 1812 & 8 \\
\hline 37 & $\mathrm{OL}-13$ & 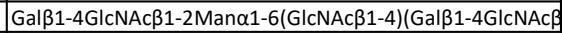 & 5 & 9 & 163 \\
\hline 38 & OL-17 & GIcNAc $\beta 1-4(G I c N A c \beta 1-2)$ Man $\alpha 1-6(G I c N A c \beta 1-4)(G I c N A c \beta 1-$ & 15986 & 1392 & 9 \\
\hline 39 & EY-10 & 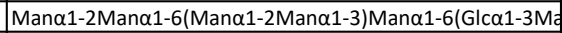 & 12 & 13 & 109 \\
\hline 40 & EY-15 & Man $\alpha 1-6($ Man $\alpha 1-3)$ Man $\alpha 1-6($ GIcNAc $\beta 1-4)(G I c N A c \beta 1-2 M a r$ & 2 & 3 & 147 \\
\hline 41 & EY-16 & GIcNAc $\beta 1-2 M a n \alpha 1-6(G I c N A c \beta 1-2 M a n \alpha 1-3)$ Man $\beta 1-4 G I c N A$ & 0 & 9 & 3400 \\
\hline 42 & EY-26 & GlcNAc $\beta 1-2 M a n \alpha 1-6(G l c N A c \beta 1-4)(G l c N A c \beta 1-2 M a n \alpha 1-3) M$ & 3 & 6 & 209 \\
\hline 43 & EY-32 & GlcNAc $\beta 1-6($ GIcNAc $\beta 1-4)(G \mid c N A c \beta 1-2)$ Man $\alpha 1-6($ GlcNAc $\beta 1-$ & 10555 & 792 & 8 \\
\hline 44 & $2^{\prime}-\mathrm{FL}$ & Fuc $\alpha 1-2$ Gal $\beta 1-4 G l c$ & 5 & 3 & 54 \\
\hline 45 & 6'-SL & 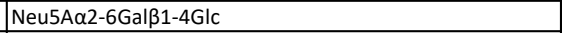 & -1 & 5 & -410 \\
\hline 46 & EY-20 & 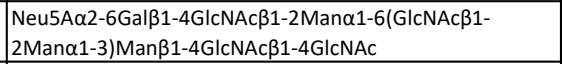 & 0 & 6 & \#DIV/0! \\
\hline 47 & Man8 & 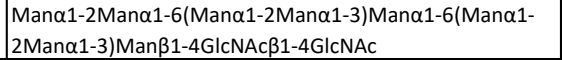 & 3 & 5 & 192 \\
\hline 48 & FBS-1 & $\begin{array}{l}\text { Neu5A } \alpha 2-3 \text { Gal } \beta 1-4 G I c N A c \beta 1-2 M a n \alpha 1-6(\text { Neu5A } \alpha 2-3 G a l \beta 1- \\
\text { 4GIcNAc } \beta 1-4((N e u 5 A \alpha 2-3 G a l \beta 1-4 G I c N A c \beta 1-2) \text { Man } \alpha 1- \\
\text { 3)Man } \beta 1-4 G I c N A c \beta 1-4 G I c N A c\end{array}$ & 1 & 4 & 757 \\
\hline 49 & Water & & 3 & 7 & 224 \\
\hline 50 & Water & & 4 & 2 & 49 \\
\hline 51 & Water & & 0 & 7 & 2946 \\
\hline 52 & Biotin-BSA & & 8979 & 1800 & 20 \\
\hline
\end{tabular}


Table S3. Printed microarray raw data. Column A to C list the ID, name and structure of printed glycans; Column D is the average relative fluorescent units (RFU); Column E is the standard deviation of 4 printed spots; Column F is the ratio between STDEV and average RFU.

\begin{tabular}{|c|c|c|c|c|c|}
\hline \multicolumn{6}{|c|}{ PNA: $10 \mathrm{ug} / \mathrm{mL}$} \\
\hline Glycan ID & Glycan name & Structure & Average & STDEV & $\% \mathrm{CV}$ \\
\hline 1 & Lacto-N-tetraose (LNT) & Galß1-3GIcNacß1-3Galß1-4GIc & 302 & 100 & 33 \\
\hline 2 & Lacto-N-neotetraose (LNnT / neo-LNT) & Galß1-4GIcNAcß1-3Galß1-4GIc & 7 & 49 & 679 \\
\hline 3 & Blood group $\mathrm{H}$ antigen pentaose type 2 / Lacto-N-neofucopentaose I (LnNFP I) & Fuc $\alpha 1-2$ Gal $\beta 1-4 G I c N A c \beta 1-3 G a l \beta 1-4 G I c$ & -6 & 23 & -364 \\
\hline 4 & Blood group A Lewisb antigen pentaose type 1 & GalNAc $\alpha 1-3$ (Fuc $\alpha 1-2$ )Galß1-3(Fuc $\alpha 1-4)$ GIcNAc & 4 & 6 & 151 \\
\hline 5 & Blood group A LewisY antigen pentaose type 2 & GaINAc $\alpha 1-3$ (Fuca1-2)Galß1-4(Fuc $\alpha 1-3)$ GIcNAc & 10 & 6 & 65 \\
\hline 6 & Blood group $\mathrm{A}$ antigen pentaose type 1 & GalNAc $\alpha 1-3($ Fuc $\alpha 1-2)$ Gal $\beta 1-3 G I c N A c \beta 1-3 G a l$ & 10 & 4 & 40 \\
\hline 7 & Blood group $\mathrm{A}$ antigen pentaose type 2 & GalNAca1-3(Fuc $\alpha 1-2)$ Galß1-4GIcNAc $\beta 1-3 G a l$ & 8 & 5 & 59 \\
\hline 8 & Blood group $A$ antigen hexaose type 1 & GalNAc $\alpha 1-3$ (Fuc $\alpha 1-2)$ Gal $\beta 1-3 G I c N A c \beta 1-3 G a l \beta 1-4 G I c$ & 7 & 8 & 112 \\
\hline 9 & Blood group $\mathrm{A}$ antigen hexaose type 2 & GalNAc $\alpha 1-3$ (Fuc $\alpha 1-2)$ Gal $\beta 1-4 G I c N A c \beta 1-3 G a l \beta 1-4 G I c$ & 2 & 4 & 205 \\
\hline 10 & Blood group B Lewisb antigen pentaose type 1 & Gal $\alpha 1-3$ (Fuc $\alpha 1-2)$ Galß1-3(Fuc $\alpha 1-4) G I c N A c$ & 5 & 5 & 105 \\
\hline 11 & Blood group B LewisY antigen pentaose type 2 & Gal $\alpha 1-3$ (Fuc $\alpha 1-2$ )Galß1-4(Fuc $\alpha 1-3)$ GIcNAc & 6 & 6 & 107 \\
\hline 12 & Blood group B antigen pentaose type 1 & 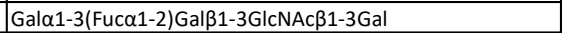 & 0 & 3 & 1194 \\
\hline 13 & Blood group B antigen pentaose type 2 & 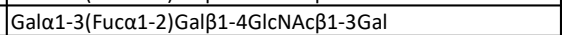 & 4 & 4 & 110 \\
\hline 14 & Blood group B antigen hexaose type 1 & Gal $\alpha 1-3$ (Fuc $\alpha 1-2)$ Galß1-3GIcNAc $\beta 1-3 G a|\beta 1-4 G| c$ & 7 & 1 & 20 \\
\hline 15 & Blood group B antigen hexaose type 2 & Gal $\alpha 1-3($ Fuc $\alpha 1-2)$ Galß1-4GIcNAc $\beta 1-3 G a l \beta 1-4 G l c$ & -2 & 3 & -189 \\
\hline 16 & LewisX (LeX) tetraose & Galß1-4( Fuc $\alpha 1-3)$ GIcNAc $\beta 1-3 G a l$ & 3 & 4 & 119 \\
\hline 17 & LewisY (LeY) pentaose & Fuc $\alpha 1-2$ Gal $\beta 1-4$ (Fuc $\alpha 1-3)$ GlcNAc $\beta 1-3 G a l$ & 7 & 4 & 61 \\
\hline 18 & Lewisa (Lea) tetraose & Gal $\beta 1-3$ (Fuc $\alpha 1-4)$ GIcNAc $\beta 1-3 G a l$ & 9 & 5 & 59 \\
\hline 19 & Lewisb (Leb) pentaose & Fuc $\alpha 1-2$ Gal $\beta 1-3$ (Fuc $\alpha 1-4)$ GlcNAc $\beta 1-3 G a l$ & -1 & 2 & -137 \\
\hline 20 & Galili antigen pentaose & Gal $\alpha 1-3$ Gal $\beta 1-4 G I c N A c \beta 1-3 G a l \beta 1-4 G I c$ & 2 & 5 & 222 \\
\hline 21 & Isoglobopentaose / iGb5 & 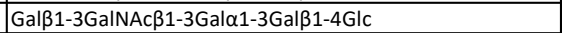 & 64969 & 251 & 0 \\
\hline 22 & Galili antigen heptaose & Gal $\alpha 1-3($ Gal$\beta 1-4 G I c N A c \beta 1-3) 2 G a|\beta 1-4 G| c$ & 22 & 32 & 144 \\
\hline 23 & Globotriaose (Gb3) / Pk antigen & Gala1-4Galß1-4GIc & 7 & 1 & 20 \\
\hline 24 & Globotetraose (Gb4) / $P$ antigen & GalNAc $\beta 1-3 G a l \alpha 1-4 G a|\beta 1-4 G| c$ & 6 & 4 & 70 \\
\hline 25 & Globo-H hexaose / Stage Specific Embryonic Antigen 3b (SSEA-3b) & Fuc $\alpha 1-2$ Gal $\beta 1-3 G a l N A c \beta 1-3 G a l \alpha 1-4 G a l \beta 1-4 G \mid c$ & 0 & 6 & -2559 \\
\hline 26 & Globo-A heptaose & 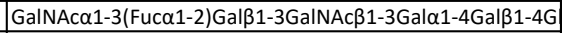 & 3 & 5 & 208 \\
\hline 27 & Globo-B heptaose & 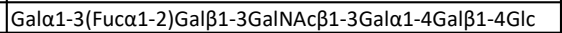 & 7 & 6 & 93 \\
\hline 28 & Blood group $\mathrm{H}$ antigen tetraose type 4 / Globo $\mathrm{H}$ tetraose & Fuc $\alpha 1-2$ Gal $\beta 1-3 G a I N A c \beta 1-3 G a l$ & 7 & 7 & 101 \\
\hline 29 & Blood group $\mathrm{A}$ antigen pentaose type 4 & 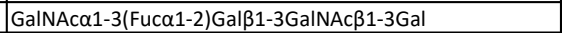 & 8 & 6 & 71 \\
\hline 30 & Blood group B antigen pentasaccharide type 4 & Gal $\alpha 1-3$ (Fuc $\alpha 1-2)$ Galß1-3GalNAc $\beta 1-3 G a l$ & 2 & 3 & 147 \\
\hline 31 & Forssman antigen pentaose & GaINAc $\alpha 1-3$ GalNAc $\beta 1-3 G a l \alpha 1-4 G a \mid \beta 1-4 G I c$ & 6 & 6 & 105 \\
\hline 32 & Isoforssman antigen pentaose & GalNAc $\alpha 1-3$ GalNAc $\beta 1-3 G a l \alpha 1-3 G a l \beta 1-4 G \mid c$ & 12 & 5 & 43 \\
\hline 33 & Globotriaose analogue type 2/ P1 antigen & Gal 1 1-4Galß1-4GIcNAc & 12 & 9 & 74 \\
\hline 34 & Isoglobo- $\mathrm{H}$ analogue type 1 & Fuc $\alpha 1-2$ Gal $\beta 1-3 G I c N A c \beta 1-3 G a l \alpha 1-3 G a l \beta 1-4 G l c$ & 5 & 6 & 113 \\
\hline 35 & OL-05 & GIcNAc $\beta 1-6($ GIcNAc $\beta 1-4)(G \mid c N A c \beta 1-2)$ Man $\alpha 1-6($ GIcNAc $\beta 1-$ & 7 & 5 & 67 \\
\hline 36 & OL-09 & Man $\alpha 1-6($ Man $\alpha 1-3)$ Man $\alpha 1-6($ GIcNAc $\beta 1-4)(G I c N A c \beta 1-4(G I C$ & 32 & 14 & 42 \\
\hline 37 & $\mathrm{OL}-13$ & 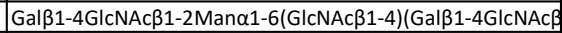 & 56 & 25 & 45 \\
\hline 38 & $\mathrm{OL}-17$ & GIcNAc $\beta 1-4(G I c N A c \beta 1-2)$ Man $\alpha 1-6(G I c N A c \beta 1-4)(G I c N A c \beta 1-$ & 374 & 117 & 31 \\
\hline 39 & EY-10 & 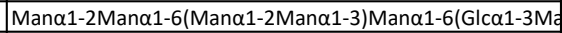 & 10 & 4 & 43 \\
\hline 40 & EY-15 & Man $\alpha 1-6($ Man $\alpha 1-3)$ Man $\alpha 1-6($ GIcNAc $\beta 1-4)(G I c N A c \beta 1-2 M a r$ & 8 & 2 & 26 \\
\hline 41 & EY-16 & GIcNAc $\beta 1-2 M a n \alpha 1-6(G I c N A c \beta 1-2 M a n \alpha 1-3)$ Man $\beta 1-4 G I c N A$ & 12 & 6 & 47 \\
\hline 42 & EY-26 & GlcNAc $\beta 1-2 M a n \alpha 1-6(G l c N A c \beta 1-4)(G l c N A c \beta 1-2 M a n \alpha 1-3) M$ & 4 & 3 & 80 \\
\hline 43 & EY-32 & GlcNAc $\beta 1-6($ GIcNAc $\beta 1-4)(G \mid c N A c \beta 1-2)$ Man $\alpha 1-6($ GlcNAc $\beta 1-$ & 785 & 186 & 24 \\
\hline 44 & $2^{\prime}-\mathrm{FL}$ & Fuc $\alpha 1-2$ Gal $\beta 1-4 G l c$ & 7 & 5 & 62 \\
\hline 45 & 6'-SL & 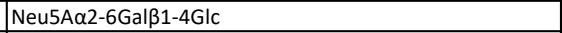 & 4 & 6 & 154 \\
\hline 46 & EY-20 & 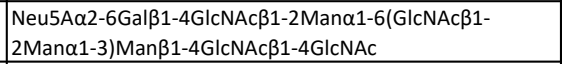 & 6 & 4 & 73 \\
\hline 47 & Man8 & 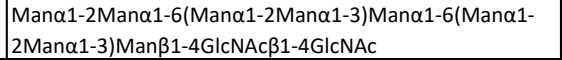 & 3 & 3 & 77 \\
\hline 48 & FBS-1 & $\begin{array}{l}\text { Neu5A } \alpha 2-3 \text { Gal } \beta 1-4 G I c N A c \beta 1-2 M a n \alpha 1-6(\text { Neu5A } \alpha 2-3 G a l \beta 1- \\
\text { 4GIcNAc } \beta 1-4((\text { Neu5A } \alpha 2-3 G a l \beta 1-4 G I c N A c \beta 1-2) M a n \alpha 1- \\
\text { 3)Man } \beta 1-4 G I c N A c \beta 1-4 G I c N A c\end{array}$ & 4 & 6 & 152 \\
\hline 49 & Water & & 0 & 4 & \#DIV/0! \\
\hline 50 & Water & & 5 & 2 & 38 \\
\hline 51 & Water & & -1 & 5 & -666 \\
\hline 52 & Biotin-BSA & & 7150 & 792 & 11 \\
\hline
\end{tabular}


Table S3. Printed microarray raw data. Column A to C list the ID, name and structure of printed glycans; Column D is the average relative fluorescent units (RFU); Column E is the standard deviation of 4 printed spots; Column F is the ratio between STDEV and average RFU.

\begin{tabular}{|c|c|c|c|c|c|}
\hline \multicolumn{6}{|c|}{ GS-I-B4: $10 \mathrm{ug} / \mathrm{mL}$} \\
\hline Glycan ID & Glycan name & Structure & Average & STDEV & $\% \mathrm{CV}$ \\
\hline 1 & Lacto-N-tetraose (LNT) & Gal $\beta 1-3 G|c N a c \beta 1-3 G a l \beta 1-4 G| c$ & 155 & 23 & 15 \\
\hline 2 & Lacto-N-neotetraose (LNnT / neo-LNT) & 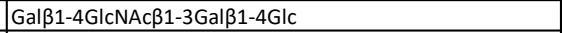 & 4 & 5 & 145 \\
\hline 3 & Blood group $\mathrm{H}$ antigen pentaose type 2 / Lacto-N-neofucopentaose I (LnNFP I) & Fuc $\alpha 1-2$ Gal $\beta 1-4$ GIcNAc $\beta 1-3 G a l \beta 1-4 G I c$ & 0 & 6 & -2386 \\
\hline 4 & Blood group A Lewisb antigen pentaose type 1 & GalNAc $\alpha 1-3$ (Fuc $\alpha 1-2$ )Galß1-3(Fuc $\alpha 1-4)$ GIcNAc & 10 & 10 & 101 \\
\hline 5 & Blood group A LewisY antigen pentaose type 2 & GalNAc $\alpha 1-3$ (Fuc $\alpha 1-2$ )Galß1-4(Fuc $\alpha 1-3)$ GIcNAc & 21 & 14 & 68 \\
\hline 6 & Blood group A antigen pentaose type 1 & 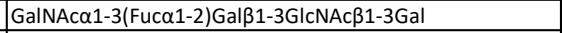 & 22 & 16 & 73 \\
\hline 7 & Blood group $\mathrm{A}$ antigen pentaose type 2 & GalNAc $\alpha 1-3($ Fuc $\alpha 1-2)$ Gal $\beta 1-4 G I c N A c \beta 1-3 G a l$ & 19 & 6 & 31 \\
\hline 8 & Blood group A antigen hexaose type 1 & GalNAc $\alpha 1-3$ (Fuc $\alpha 1-2)$ Gal $\beta 1-3$ GIcNAc $\beta 1-3 G a l \beta 1-4 G l c$ & 11 & 4 & 38 \\
\hline 9 & Blood group $A$ antigen hexaose type 2 & GalNAc $\alpha 1-3$ (Fuc $\alpha 1-2)$ Gal $\beta 1-4 G I c N A c \beta 1-3 G a l \beta 1-4 G I c$ & 7 & 5 & 78 \\
\hline 10 & Blood group B Lewisb antigen pentaose type 1 & Gal $\alpha 1-3$ (Fuc $\alpha 1-2$ )Gal $\beta 1-3$ (Fuc $\alpha 1-4)$ GlcNAc & 65375 & 81 & 0 \\
\hline 11 & Blood group B LewisY antigen pentaose type 2 & Gala1-3(Fuc $\alpha 1-2)$ Gal $\beta 1-4($ Fuc $\alpha 1-3)$ GIcNAc & 65355 & 117 & 0 \\
\hline 12 & Blood group B antigen pentaose type 1 & Gal $\alpha 1-3$ (Fuc $\alpha 1-2$ )Gal $\beta 1-3 G \mid c N A c \beta 1-3 G a l$ & 63957 & 1599 & 3 \\
\hline 13 & Blood group B antigen pentaose type 2 & Gal $\alpha 1-3$ (Fuc $\alpha 1-2$ )Gal $\beta 1-4 G \mid c N A c \beta 1-3 G a l$ & 65393 & 136 & 0 \\
\hline 14 & Blood group B antigen hexaose type 1 & Gal $\alpha 1-3$ (Fuc $\alpha 1-2)$ Gal $\beta 1-3$ GlcNAc $\beta 1-3 G a l \beta 1-4 G l c$ & 65181 & 122 & 0 \\
\hline 15 & Blood group B antigen hexaose type 2 & Gal $\alpha 1-3$ (Fuc $\alpha 1-2)$ Gal $\beta 1-4 G|c N A c \beta 1-3 G a l \beta 1-4 G| c$ & 64171 & 785 & 1 \\
\hline 16 & LewisX (LeX) tetraose & Gal $\beta 1-4$ ( Fuc $\alpha 1-3)$ GIcNAc $\beta 1-3 G a l$ & 8 & 2 & 23 \\
\hline 17 & LewisY (LeY) pentaose & Fuc $\alpha 1-2$ Gal $\beta 1-4($ Fuc $\alpha 1-3)$ GlcNAc $\beta 1-3 G a l$ & 414 & 31 & 7 \\
\hline 18 & Lewisa (Lea) tetraose & Galß1-3(Fuc $\alpha 1-4)$ GIcNAc $\beta 1-3 G a l$ & 7 & 6 & 96 \\
\hline 19 & Lewisb (Leb) pentaose & Fuc $\alpha 1-2$ Gal $\beta 1-3$ (Fuc $\alpha 1-4)$ GlcNAc $\beta 1-3 G a l$ & 955 & 66 & 7 \\
\hline 20 & Galili antigen pentaose & Gal $\alpha 1-3$ Gal $\beta 1-4 G|c N A c \beta 1-3 G a l \beta 1-4 G| c$ & 65382 & 59 & 0 \\
\hline 21 & Isoglobopentaose / iGb5 & 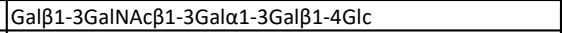 & 11 & 14 & 125 \\
\hline 22 & Galili antigen heptaose & Gal $\alpha 1-3($ Gal $\beta 1-4 G \mid c N A c \beta 1-3) 2 G a l \beta 1-4 G \mid c$ & 63605 & 2092 & 3 \\
\hline 23 & Globotriaose (Gb3) / Pk antigen & Gala1-4Galß1-4GIc & 58868 & 2918 & 5 \\
\hline 24 & Globotetraose (Gb4) / $P$ antigen & GalNAc $\beta 1-3 G a l \alpha 1-4 G a|\beta 1-4 G| c$ & 2 & 9 & 495 \\
\hline 25 & Globo-H hexaose / Stage Specific Embryonic Antigen 3b (SSEA-3b) & Fuc $\alpha 1-2$ Gal $\beta 1-3 G a l N A c \beta 1-3 G a l \alpha 1-4 G a l \beta 1-4 G l c$ & 8 & 5 & 64 \\
\hline 26 & Globo-A heptaose & 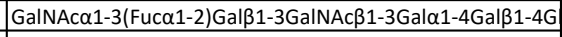 & -14 & 36 & -254 \\
\hline 27 & Globo-B heptaose & Gal $\alpha 1-3($ Fuc $\alpha 1-2)$ Galß1-3GalNAc $\beta 1-3 G a l \alpha 1-4 G a l \beta 1-4 G l c$ & 64693 & 478 & 1 \\
\hline 28 & Blood group $\mathrm{H}$ antigen tetraose type 4 / Globo $\mathrm{H}$ tetraose & Fuc $\alpha 1-2$ Gal $\beta 1-3 G a I N A c \beta 1-3 G a l$ & 6 & 5 & 92 \\
\hline 29 & Blood group A antigen pentaose type 4 & GalNAc $\alpha 1-3($ Fuc $\alpha 1-2)$ Gal $\beta 1-3 G a l N A c \beta 1-3 G a l$ & 4 & 5 & 127 \\
\hline 30 & Blood group B antigen pentasaccharide type 4 & Gal $\alpha 1-3$ (Fuc $\alpha 1-2$ )Galß1-3GalNAc $\beta 1-3 G a l$ & 65385 & 136 & 0 \\
\hline 31 & Forssman antigen pentaose & GalNAc $\alpha 1-3 G a l N A c \beta 1-3 G a l \alpha 1-4 G a l \beta 1-4 G \mid c$ & -15 & 32 & -211 \\
\hline 32 & Isoforssman antigen pentaose & GaINAc $\alpha 1-3$ GalNAc $\beta 1-3 G a l \alpha 1-3 G a l \beta 1-4 G I c$ & 6 & 8 & 143 \\
\hline 33 & Globotriaose analogue type 2/ $\mathrm{P} 1$ antigen & 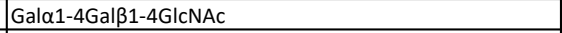 & 62635 & 1582 & 3 \\
\hline 34 & Isoglobo- $\mathrm{H}$ analogue type 1 & Fuc $\alpha 1-2$ Gal $\beta 1-3 G I c N A c \beta 1-3 G a l \alpha 1-3 G a l \beta 1-4 G l c$ & 5 & 5 & 121 \\
\hline 35 & OL-05 & GlcNAc $\beta 1-6($ GIcNAc $\beta 1-4)(G \mid c N A c \beta 1-2)$ Man $\alpha 1-6($ GlcNAc $\beta 1-$ & 501 & 45 & 9 \\
\hline 36 & OL-09 & Man $\alpha 1-6($ Man $\alpha 1-3)$ Man $\alpha 1-6($ GlcNAc $\beta 1-4)(G l c N A c \beta 1-4(G l c$ & 7013 & 930 & 13 \\
\hline 37 & $\mathrm{OL}-13$ & Gal $\beta 1-4$ GIcNAc $\beta 1-2 M a n \alpha 1-6(G \mid c N A c \beta 1-4)(G a l \beta 1-4 G \mid c N A c \beta$ & 1456 & 27 & 2 \\
\hline 38 & OL-17 & GlcNAc $\beta 1-4(G I c N A c \beta 1-2) M a n \alpha 1-6(G l c N A c \beta 1-4)(G l c N A c \beta 1-$ & 13864 & 575 & 4 \\
\hline 39 & EY-10 & 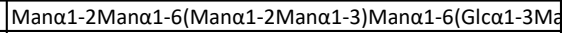 & 21 & 13 & 60 \\
\hline 40 & EY-15 & Man $\alpha 1-6($ Man $\alpha 1-3)$ Man $\alpha 1-6($ GlcNAc $\beta 1-4)(G l c N A c \beta 1-2 M a r$ & 47 & 19 & 41 \\
\hline 41 & EY-16 & GlcNAc $\beta 1-2 M a n \alpha 1-6(G l c N A c \beta 1-2 M a n \alpha 1-3)$ Man $\beta 1-4 G I c N A$ & 433 & 53 & 12 \\
\hline 42 & EY-26 & GlcNAc $\beta 1-2 M a n \alpha 1-6(G l c N A c \beta 1-4)(G l c N A c \beta 1-2 M a n \alpha 1-3) M$ & 309 & 26 & 9 \\
\hline 43 & EY-32 & GlcNAc $\beta 1-6(G I c N A c \beta 1-4)(G l c N A c \beta 1-2) M a n \alpha 1-6(G l c N A c \beta 1-$ & 8103 & 219 & 3 \\
\hline 44 & 2'-FL & Fuc $\alpha 1-2$ Galß1-4Glc & 0 & 3 & 1281 \\
\hline 45 & 6'-SL & 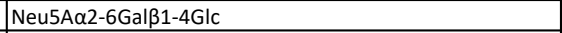 & 7 & 5 & 74 \\
\hline 46 & EY-20 & $\begin{array}{l}\text { Neu5A } \alpha 2-6 \text { Gal } \beta 1-4 G \mid c N A c \beta 1-2 M a n \alpha 1-6 \text { (GIcNAc } \beta 1- \\
\text { 2Man } \alpha 1-3) \text { Man } \beta 1-4 G l c N A c \beta 1-4 G I c N A c\end{array}$ & 11 & 10 & 91 \\
\hline 47 & Man8 & 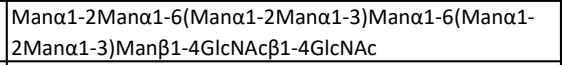 & 40 & 33 & 84 \\
\hline 48 & FBS-1 & 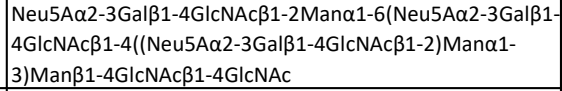 & 1 & 5 & 360 \\
\hline 49 & Water & & -1 & 3 & -441 \\
\hline 50 & Water & & 4 & 7 & 163 \\
\hline 51 & Water & & -1 & 4 & -515 \\
\hline 52 & Biotin-BSA & & 8551 & 2380 & 28 \\
\hline
\end{tabular}


Table S3. Printed microarray raw data. Column A to C list the ID, name and structure of printed glycans; Column D is the average relative fluorescent units (RFU); Column E is the standard deviation of 4 printed spots; Column F is the ratio between STDEV and average RFU.

\begin{tabular}{|c|c|c|c|c|c|}
\hline \multicolumn{6}{|c|}{ BPL: $10 \mathrm{ug} / \mathrm{mL}$} \\
\hline Glycan ID & Glycan name & Structure & Average & STDEV & $\% \mathrm{CV}$ \\
\hline 1 & Lacto-N-tetraose (LNT) & Galß1-3GlcNacß1-3Galß1-4Glc & 65092 & 320 & 0 \\
\hline 2 & Lacto-N-neotetraose (LNnT / neo-LNT) & Galß1-4GIcNAcß1-3Galß1-4GIc & 65462 & 39 & 0 \\
\hline 3 & Blood group $\mathrm{H}$ antigen pentaose type 2 / Lacto-N-neofucopentaose I (LnNFP I) & Fuc $\alpha 1-2$ Gal $\beta 1-4$ GIcNAc $\beta 1-3 G a l \beta 1-4 G I c$ & 102 & 189 & 186 \\
\hline 4 & Blood group A Lewisb antigen pentaose type 1 & GalNAc $\alpha 1-3$ (Fuc $\alpha 1-2$ )Galß1-3(Fuc $\alpha 1-4)$ GIcNAc & 13 & 6 & 45 \\
\hline 5 & Blood group A LewisY antigen pentaose type 2 & GaINAc $\alpha 1-3$ (Fuc $\alpha 1-2$ )Gal $\beta 1-4$ (Fuc $\alpha 1-3)$ GIcNAc & 21 & 9 & 45 \\
\hline 6 & Blood group $\mathrm{A}$ antigen pentaose type 1 & 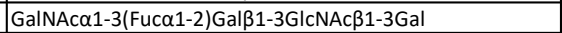 & 24 & 36 & 151 \\
\hline 7 & Blood group $A$ antigen pentaose type 2 & GalNAc $\alpha 1-3$ (Fuc $\alpha 1-2)$ Galß1-4GIcNAc $\beta 1-3 G a l$ & 1 & 9 & 1800 \\
\hline 8 & Blood group $A$ antigen hexaose type 1 & GalNAc $\alpha 1-3$ (Fuc $\alpha 1-2)$ Gal $\beta 1-3 G I c N A c \beta 1-3 G a l \beta 1-4 G I c$ & 7 & 8 & 116 \\
\hline 9 & Blood group $\mathrm{A}$ antigen hexaose type 2 & GalNAc $\alpha 1-3$ (Fuc $\alpha 1-2)$ Gal $\beta 1-4 G|c N A c \beta 1-3 G a l \beta 1-4 G| c$ & 5 & 11 & 220 \\
\hline 10 & Blood group B Lewisb antigen pentaose type 1 & Gal $\alpha 1-3$ (Fuc $\alpha 1-2)$ Gal $\beta 1-3$ (Fuc $\alpha 1-4)$ GIcNAc & -25 & 4 & -17 \\
\hline 11 & Blood group B LewisY antigen pentaose type 2 & Gal $\alpha 1-3$ (Fuc $\alpha 1-2$ )Gal $\beta 1-4($ Fuc $\alpha 1-3)$ GlcNAc & -13 & 4 & -33 \\
\hline 12 & Blood group B antigen pentaose type 1 & 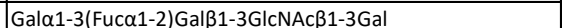 & 12 & 13 & 113 \\
\hline 13 & Blood group B antigen pentaose type 2 & 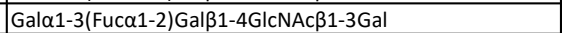 & 2 & 9 & 602 \\
\hline 14 & Blood group B antigen hexaose type 1 & Gal $\alpha 1-3$ (Fuc $\alpha 1-2)$ Galß1-3GIcNAc $\beta 1-3 G a|\beta 1-4 G| c$ & -12 & 5 & -40 \\
\hline 15 & Blood group B antigen hexaose type 2 & Gal $\alpha 1-3$ (Fuc $\alpha 1-2)$ Gal $\beta 1-4 G|c N A c \beta 1-3 G a l \beta 1-4 G| c$ & -13 & 6 & -45 \\
\hline 16 & LewisX (LeX) tetraose & Galß1-4( Fuc $\alpha 1-3)$ GIcNAc $\beta 1-3 G a l$ & 65344 & 100 & 0 \\
\hline 17 & LewisY (LeY) pentaose & Fuc $\alpha 1-2$ Gal $\beta 1-4($ Fuc $\alpha 1-3)$ GIcNAc $\beta 1-3 G a l$ & 31 & 8 & 27 \\
\hline 18 & Lewisa (Lea) tetraose & Galß1-3(Fuc $\alpha 1-4)$ GIcNAc $\beta 1-3 G a l$ & 62160 & 2649 & 4 \\
\hline 19 & Lewisb (Leb) pentaose & 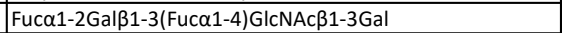 & -11 & 5 & -44 \\
\hline 20 & Galili antigen pentaose & Gal $\alpha 1-3 G a l \beta 1-4 G|c N A c \beta 1-3 G a l \beta 1-4 G| c$ & 65449 & 110 & 0 \\
\hline 21 & Isoglobopentaose / iGb5 & 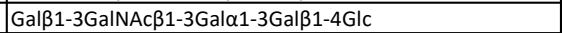 & 65265 & 149 & 0 \\
\hline 22 & Galili antigen heptaose & Gal $\alpha 1-3($ Gal$\beta 1-4 G I c N A c \beta 1-3) 2$ Gal $\beta 1-4 G \mid c$ & 65430 & 60 & 0 \\
\hline 23 & Globotriaose (Gb3) / Pk antigen & 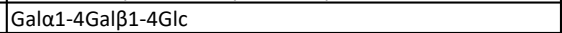 & -10 & 6 & -63 \\
\hline 24 & Globotetraose (Gb4) / P antigen & GalNAcß1-3Gal $\alpha 1-4 G a|\beta 1-4 G| c$ & 65202 & 252 & 0 \\
\hline 25 & Globo-H hexaose / Stage Specific Embryonic Antigen 3b (SSEA-3b) & Fuc $\alpha 1-2$ Gal $\beta 1-3 G a l N A c \beta 1-3 G a l \alpha 1-4 G a l \beta 1-4 G \mid c$ & 16 & 7 & 44 \\
\hline 26 & Globo-A heptaose & 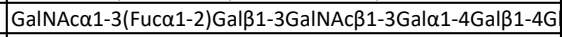 & -27 & 26 & -99 \\
\hline 27 & Globo-B heptaose & 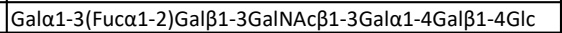 & -14 & 31 & -223 \\
\hline 28 & Blood group $\mathrm{H}$ antigen tetraose type 4 / Globo $\mathrm{H}$ tetraose & Fuc $\alpha 1-2$ Gal $\beta 1-3 G$ GINAc $\beta 1-3 G a l$ & 15 & 7 & 46 \\
\hline 29 & Blood group $\mathrm{A}$ antigen pentaose type 4 & 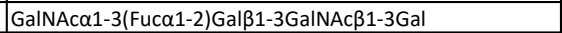 & 3 & 11 & 356 \\
\hline 30 & Blood group B antigen pentasaccharide type 4 & 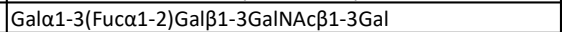 & -20 & 29 & -148 \\
\hline 31 & Forssman antigen pentaose & GaINAc $\alpha 1-3 G a I N A c \beta 1-3 G a l \alpha 1-4 G a \mid \beta 1-4 G I c$ & 11785 & 596 & 5 \\
\hline 32 & Isoforssman antigen pentaose & GaINAc $\alpha 1-3$ GalNAc $\beta 1-3 G a l \alpha 1-3 G a l \beta 1-4 G I c$ & 15472 & 1588 & 10 \\
\hline 33 & Globotriaose analogue type 2/ P1 antigen & Gal 1 1-4Galß1-4GIcNAc & 17 & 3 & 16 \\
\hline 34 & Isoglobo- $\mathrm{H}$ analogue type 1 & Fuc $\alpha 1-2$ Gal $\beta 1-3 G|c N A c \beta 1-3 G a l \alpha 1-3 G a l \beta 1-4 G| c$ & 7 & 2 & 32 \\
\hline 35 & OL-05 & GIcNAc $\beta 1-6($ GIcNAc $\beta 1-4)(G \mid c N A c \beta 1-2)$ Man $\alpha 1-6($ GIcNAc $\beta 1-$ & 17 & 6 & 38 \\
\hline 36 & OL-09 & Man $\alpha 1-6($ Man $\alpha 1-3)$ Man $\alpha 1-6($ GlcNAc$\beta 1-4)(G I c N A c \beta 1-4(G I C$ & 14471 & 1566 & 11 \\
\hline 37 & $\mathrm{OL}-13$ & 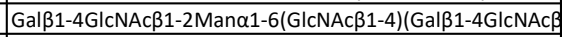 & 64945 & 435 & 1 \\
\hline 38 & OL-17 & GIcNAc $\beta 1-4($ GIcNAc $\beta 1-2)$ Man $\alpha 1-6(G I c N A c \beta 1-4)(G I c N A c \beta 1-$ & 2038 & 101 & 5 \\
\hline 39 & EY-10 & 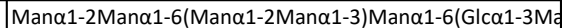 & 13 & 7 & 52 \\
\hline 40 & EY-15 & Man $\alpha 1-6($ Man $\alpha 1-3)$ Man $\alpha 1-6($ GIcNAc $\beta 1-4)(G I c N A c \beta 1-2 M a r$ & 176 & 129 & 73 \\
\hline 41 & EY-16 & GIcNAc $\beta 1-2$ Man $\alpha 1-6($ GIcNAc $\beta 1-2 M a n \alpha 1-3)$ Man $\beta 1-4 G I c N A$ & 21 & 7 & 31 \\
\hline 42 & EY-26 & GIcNAc $\beta 1-2 M a n \alpha 1-6(G I c N A c \beta 1-4)(G I c N A c \beta 1-2 M a n \alpha 1-3) M$ & 781 & 162 & 21 \\
\hline 43 & EY-32 & GIcNAc $\beta 1-6($ GIcNAc $\beta 1-4)(G \mid c N A c \beta 1-2)$ Man $\alpha 1-6($ GIcNAc $\beta 1-$ & 1143 & 153 & 13 \\
\hline 44 & $2^{\prime}-\mathrm{FL}$ & Fuco1-2Galß1-4Glc & 5 & 1 & 28 \\
\hline 45 & 6'-SL & 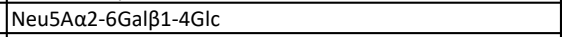 & 654 & 106 & 16 \\
\hline 46 & EY-20 & 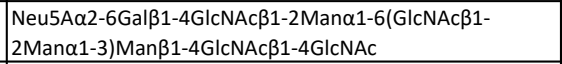 & 9 & 3 & 35 \\
\hline 47 & Man8 & 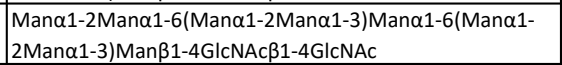 & 7 & 4 & 56 \\
\hline 48 & FBS-1 & $\begin{array}{l}\text { Neu5A } \alpha 2-3 \text { Gal } \beta 1-4 G I c N A c \beta 1-2 M a n \alpha 1-6(\text { Neu5A } \alpha 2-3 G a l \beta 1- \\
\text { 4GIcNAc } \beta 1-4((\text { Neu5A } \alpha 2-3 G a l \beta 1-4 G I c N A c \beta 1-2) M a n \alpha 1- \\
\text { 3)Man } \beta 1-4 G I c N A c \beta 1-4 G I c N A c\end{array}$ & 212 & 8 & 4 \\
\hline 49 & Water & & 3 & 6 & 239 \\
\hline 50 & Water & & 6 & 3 & 46 \\
\hline 51 & Water & & -3 & 6 & -230 \\
\hline 52 & Biotin-BSA & & 7722 & 1050 & 14 \\
\hline
\end{tabular}


Table S4. Printed microarray raw data. Column A to C list the ID, name and structure of printed glycans; Column D is the average relative fluorescent units (RFU); Column E is the standard deviation of 4 printed spots; Column $\mathrm{F}$ is the ratio between STDEV and average RFU.

\begin{tabular}{|c|c|c|c|c|c|}
\hline \multicolumn{6}{|c|}{ ConA: $0.001 \mathrm{ug} / \mathrm{mL}$} \\
\hline Glycan ID & Glycan name & Structure & Average & STDEV & $\% \mathrm{CV}$ \\
\hline 1 & Lacto-N-tetraose (LNT) & Galß1-3GIcNac $\beta 1-3 G a l \beta 1-4 G \mid c$ & 147 & 33 & 22 \\
\hline 2 & Lacto-N-neotetraose (LNnT / neo-LNT) & 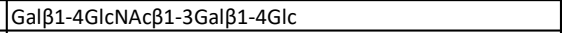 & -30 & 130 & -429 \\
\hline 3 & Blood group $\mathrm{H}$ antigen pentaose type 2 / Lacto-N-neofucopentaose I (LnNFP I) & Fuc $\alpha 1-2$ Gal $\beta 1-4$ GlcNAc $\beta 1-3 G a \mid \beta 1-4 G l c$ & -17 & 105 & -606 \\
\hline 4 & Blood group A Lewisb antigen pentaose type 1 & GalNAc $\alpha 1-3$ (Fuc $\alpha 1-2$ )Galß1-3(Fuc $\alpha 1-4)$ GIcNAc & 61 & 26 & 42 \\
\hline 5 & Blood group A LewisY antigen pentaose type 2 & GalNAc $\alpha 1-3$ (Fuc $\alpha 1-2$ )Galß1-4(Fuc $\alpha 1-3)$ GIcNAc & 5 & 6 & 136 \\
\hline 6 & Blood group A antigen pentaose type 1 & 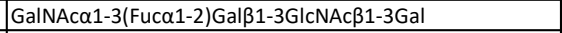 & 15 & 13 & 87 \\
\hline 7 & Blood group $\mathrm{A}$ antigen pentaose type 2 & GalNAc $\alpha 1-3($ Fuc $\alpha 1-2)$ Gal $\beta 1-4 G I c N A c \beta 1-3 G a l$ & 8 & 12 & 155 \\
\hline 8 & Blood group A antigen hexaose type 1 & GalNAc $\alpha 1-3$ (Fuc $\alpha 1-2)$ Gal $\beta 1-3$ GIcNAc $\beta 1-3 G a l \beta 1-4 G l c$ & -9 & 15 & -170 \\
\hline 9 & Blood group $\mathrm{A}$ antigen hexaose type 2 & GalNAc $\alpha 1-3$ (Fuc $\alpha 1-2)$ Gal $\beta 1-4$ GIcNAc $\beta 1-3 G a l \beta 1-4 G \mid c$ & 6 & 13 & 223 \\
\hline 10 & Blood group B Lewisb antigen pentaose type 1 & Gal $\alpha 1-3$ (Fuc $\alpha 1-2$ )Gal $\beta 1-3$ (Fuc $\alpha 1-4)$ GlcNAc & 5 & 14 & 293 \\
\hline 11 & Blood group B LewisY antigen pentaose type 2 & Gal $\alpha 1-3$ (Fuc $\alpha 1-2$ )Gal $\beta 1-4$ (Fuc $\alpha 1-3)$ GlcNAc & 79 & 12 & 15 \\
\hline 12 & Blood group B antigen pentaose type 1 & Gal $\alpha 1-3$ (Fuc $\alpha 1-2$ )Gal $\beta 1-3 G \mid c N A c \beta 1-3 G a l$ & -13 & 46 & -351 \\
\hline 13 & Blood group B antigen pentaose type 2 & Gal $\alpha 1-3$ (Fuc $\alpha 1-2$ )Gal $\beta 1-4 G \mid c N A c \beta 1-3 G a l$ & 13 & 19 & 155 \\
\hline 14 & Blood group B antigen hexaose type 1 & Gal $\alpha 1-3$ (Fuc $\alpha 1-2)$ Gal $\beta 1-3$ GlcNAc $\beta 1-3 G a l \beta 1-4 G l c$ & -5 & 7 & -149 \\
\hline 15 & Blood group B antigen hexaose type 2 & Gal $\alpha 1-3$ (Fuc $\alpha 1-2)$ Gal $\beta 1-4 G|c N A c \beta 1-3 G a l \beta 1-4 G| c$ & 4 & 5 & 137 \\
\hline 16 & LewisX (LeX) tetraose & Gal $\beta 1-4$ ( Fuc $\alpha 1-3)$ GIcNAc $\beta 1-3 G a l$ & 58 & 158 & 274 \\
\hline 17 & LewisY (LeY) pentaose & Fuc $\alpha 1-2$ Gal $\beta 1-4($ Fuc $\alpha 1-3)$ GlcNAc $\beta 1-3 G a l$ & 54 & 25 & 47 \\
\hline 18 & Lewisa (Lea) tetraose & Galß1-3(Fuc $\alpha 1-4)$ GIcNAc $\beta 1-3 G a l$ & 14 & 8 & 54 \\
\hline 19 & Lewisb (Leb) pentaose & Fuc $\alpha 1-2$ Gal $\beta 1-3$ (Fuc $\alpha 1-4)$ GlcNAc $\beta 1-3 G a l$ & 6 & 5 & 99 \\
\hline 20 & Galili antigen pentaose & Gal $\alpha 1-3 G a l \beta 1-4 G|c N A c \beta 1-3 G a l \beta 1-4 G| c$ & 2 & 69 & 3078 \\
\hline 21 & Isoglobopentaose / iGb5 & Gal $\beta 1-3 G a l N A c \beta 1-3 G a l \alpha 1-3 G a l \beta 1-4 G l c$ & 3 & 10 & 399 \\
\hline 22 & Galili antigen heptaose & Gal $\alpha 1-3($ Gal$\beta 1-4 G \mid c N A c \beta 1-3) 2 G a l \beta 1-4 G \mid c$ & 6 & 12 & 225 \\
\hline 23 & Globotriaose (Gb3) / Pk antigen & Gala1-4Galß1-4GIc & 2 & 4 & 200 \\
\hline 24 & Globotetraose (Gb4) / $P$ antigen & GalNAc $\beta 1-3 G a l \alpha 1-4 G a|\beta 1-4 G| c$ & 5 & 11 & 218 \\
\hline 25 & Globo-H hexaose / Stage Specific Embryonic Antigen 3b (SSEA-3b) & Fuc $\alpha 1-2$ Gal $\beta 1-3 G a l N A c \beta 1-3 G a l \alpha 1-4 G a l \beta 1-4 G l c$ & 1 & 9 & 683 \\
\hline 26 & Globo-A heptaose & 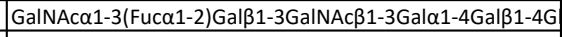 & 7 & 10 & 140 \\
\hline 27 & Globo-B heptaose & Gal $\alpha 1-3($ Fuc $\alpha 1-2)$ Galß1-3GalNAc $\beta 1-3 G a l \alpha 1-4 G a l \beta 1-4 G l c$ & 4 & 10 & 275 \\
\hline 28 & Blood group $\mathrm{H}$ antigen tetraose type 4 / Globo $\mathrm{H}$ tetraose & Fuc $\alpha 1-2$ Gal $\beta 1-3 G a I N A c \beta 1-3 G a l$ & -8 & 3 & -30 \\
\hline 29 & Blood group A antigen pentaose type 4 & GalNAc $\alpha 1-3($ Fuc $\alpha 1-2)$ Gal $\beta 1-3 G a l N A c \beta 1-3 G a l$ & -5 & 10 & -189 \\
\hline 30 & Blood group B antigen pentasaccharide type 4 & Gal $\alpha 1-3$ (Fuc $\alpha 1-2$ )Galß1-3GalNAc $\beta 1-3 G a l$ & -4 & 2 & -40 \\
\hline 31 & Forssman antigen pentaose & GalNAc $\alpha 1-3$ GalNAc $\beta 1-3 G a l \alpha 1-4 G a l \beta 1-4 G l c$ & -5 & 5 & -110 \\
\hline 32 & Isoforssman antigen pentaose & GaINAc $\alpha 1-3$ GalNAc $\beta 1-3 G a l \alpha 1-3 G a l \beta 1-4 G I c$ & -1 & 5 & -360 \\
\hline 33 & Globotriaose analogue type 2/ $\mathrm{P} 1$ antigen & 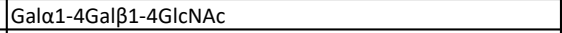 & 4 & 6 & 159 \\
\hline 34 & Isoglobo- $\mathrm{H}$ analogue type 1 & Fuc $\alpha 1-2$ Gal $\beta 1-3 G I c N A c \beta 1-3 G a l \alpha 1-3 G a l \beta 1-4 G l c$ & 17 & 4 & 25 \\
\hline 35 & OL-05 & GlcNAc $\beta 1-6($ GIcNAc $\beta 1-4)(G \mid c N A c \beta 1-2)$ Man $\alpha 1-6($ GlcNAc $\beta 1-$ & 5 & 4 & 71 \\
\hline 36 & OL-09 & Man $\alpha 1-6($ Man $\alpha 1-3)$ Man $\alpha 1-6($ GlcNAc $\beta 1-4)(G l c N A c \beta 1-4(G l c$ & 261 & 55 & 21 \\
\hline 37 & $\mathrm{OL}-13$ & 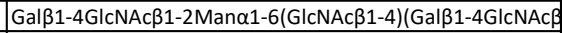 & 12 & 4 & 37 \\
\hline 38 & OL-17 & GlcNAc $\beta 1-4(G I c N A c \beta 1-2) M a n \alpha 1-6(G l c N A c \beta 1-4)(G l c N A c \beta 1-$ & -3 & 3 & -105 \\
\hline 39 & EY-10 & 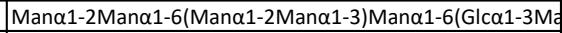 & 1987 & 159 & 8 \\
\hline 40 & EY-15 & Man $\alpha 1-6($ Man $\alpha 1-3)$ Man $\alpha 1-6($ GlcNAc $\beta 1-4)(G l c N A c \beta 1-2 M a r$ & 1213 & 223 & 18 \\
\hline 41 & EY-16 & GlcNAc $\beta 1-2 M a n \alpha 1-6(G l c N A c \beta 1-2 M a n \alpha 1-3)$ Man $\beta 1-4 G I c N A$ & 113 & 8 & 7 \\
\hline 42 & EY-26 & GIcNAc $\beta 1-2$ Man $\alpha 1-6($ GlcNAc $\beta 1-4)(G I c N A c \beta 1-2 M a n \alpha 1-3) M$ & 161 & 11 & 7 \\
\hline 43 & EY-32 & GlcNAc $\beta 1-6(G I c N A c \beta 1-4)(G l c N A c \beta 1-2) M a n \alpha 1-6(G l c N A c \beta 1-$ & -4 & 7 & -174 \\
\hline 44 & 2'-FL & Fuc $\alpha 1-2$ Galß1-4Glc & 135 & 26 & 19 \\
\hline 45 & 6'-SL & 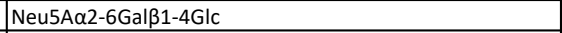 & -9 & 5 & -57 \\
\hline 46 & EY-20 & $\begin{array}{l}\text { Neu5A } \alpha 2-6 \text { Gal } \beta 1-4 G \mid c N A c \beta 1-2 M a n \alpha 1-6 \text { (GIcNAc } \beta 1- \\
\text { 2Man } \alpha 1-3) \text { Man } \beta 1-4 G l c N A c \beta 1-4 G I c N A c\end{array}$ & 27 & 7 & 26 \\
\hline 47 & Man8 & 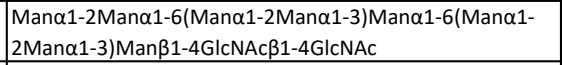 & 2493 & 150 & 6 \\
\hline 48 & FBS-1 & 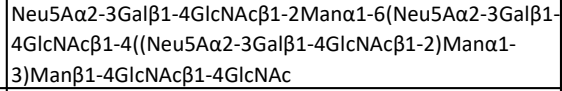 & -4 & 7 & -187 \\
\hline 49 & Water & & -4 & 3 & -84 \\
\hline 50 & Water & & -5 & 6 & -127 \\
\hline 51 & Water & & -6 & 5 & -82 \\
\hline 52 & Biotin-BSA & & 14653 & 2758 & 19 \\
\hline
\end{tabular}


Table S4. Printed microarray raw data. Column A to C list the ID, name and structure of printed glycans; Column D is the average relative fluorescent units (RFU); Column E is the standard deviation of 4 printed spots; Column $\mathrm{F}$ is the ratio between STDEV and average RFU.

\begin{tabular}{|c|c|c|c|c|c|}
\hline \multicolumn{6}{|c|}{ ConA: $0.01 \mathrm{ug} / \mathrm{mL}$} \\
\hline Glycan ID & Glycan name & Structure & Average & STDEV & $\% \mathrm{CV}$ \\
\hline 1 & Lacto-N-tetraose (LNT) & Gal $\beta 1-3 G|c N a c \beta 1-3 G a l \beta 1-4 G| c$ & 491 & 126 & 26 \\
\hline 2 & Lacto-N-neotetraose (LNnT / neo-LNT) & Galß1-4GIcNAcß1-3Galß1-4GIc & 81 & 32 & 40 \\
\hline 3 & Blood group H antigen pentaose type 2 / Lacto-N-neofucopentaose I (LnNFP I) & Fuc $\alpha 1-2$ Gal $\beta 1-4 G I c N A c \beta 1-3 G a l \beta 1-4 G I c$ & 69 & 26 & 37 \\
\hline 4 & Blood group A Lewisb antigen pentaose type 1 & GalNAc $\alpha 1-3$ (Fuc $\alpha 1-2)$ Galß1-3(Fuc $\alpha 1-4)$ GIcNAc & 76 & 14 & 18 \\
\hline 5 & Blood group A LewisY antigen pentaose type 2 & GalNAca1-3(Fuc $\alpha 1-2)$ Gal $\beta 1-4($ Fuc $\alpha 1-3)$ GIcNAc & 14 & 15 & 103 \\
\hline 6 & Blood group A antigen pentaose type 1 & GalNAc $\alpha 1-3$ (Fuc $\alpha 1-2)$ Gal $\beta 1-3 G I c N A c \beta 1-3 G a l$ & 12 & 17 & 138 \\
\hline 7 & Blood group $\mathrm{A}$ antigen pentaose type 2 & 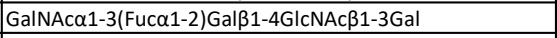 & 18 & 4 & 24 \\
\hline 8 & Blood group $\mathrm{A}$ antigen hexaose type 1 & GalNAc $\alpha 1-3$ (Fuc $\alpha 1-2$ )Gal $\beta 1-3$ GIcNAc $\beta 1-3 G a l \beta 1-4 G \mid c$ & 22 & 5 & 21 \\
\hline 9 & Blood group $A$ antigen hexaose type 2 & GalNAc $\alpha 1-3$ (Fuc $\alpha 1-2)$ Gal $\beta 1-4 G|c N A c \beta 1-3 G a l \beta 1-4 G| c$ & 4 & 6 & 151 \\
\hline 10 & Blood group B Lewisb antigen pentaose type 1 & 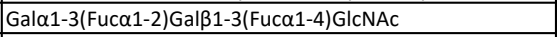 & -15 & 23 & -159 \\
\hline 11 & Blood group B LewisY antigen pentaose type 2 & Gal $\alpha 1-3$ (Fuc $\alpha 1-2)$ Galß1-4(Fuc $\alpha 1-3)$ GlcNAc & 41 & 6 & 14 \\
\hline 12 & Blood group B antigen pentaose type 1 & Gal $\alpha 1-3$ (Fuc $\alpha 1-2)$ Gal $\beta 1-3 G \mid c N A c \beta 1-3 G a l$ & 8 & 4 & 57 \\
\hline 13 & Blood group B antigen pentaose type 2 & 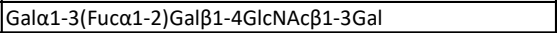 & 8 & 5 & 68 \\
\hline 14 & Blood group B antigen hexaose type 1 & Gal $\alpha 1-3$ (Fuc $\alpha 1-2)$ Gal $\beta 1-3$ GIcNAc $\beta 1-3 G a l \beta 1-4 G l c$ & 14 & 9 & 66 \\
\hline 15 & Blood group B antigen hexaose type 2 & Gal $\alpha 1-3$ (Fuc $\alpha 1-2)$ Gal $\beta 1-4$ GIcNAc $\beta 1-3 G a l \beta 1-4 G l c$ & -3 & 8 & -253 \\
\hline 16 & LewisX (LeX) tetraose & Galß1-4( Fuc $\alpha 1-3)$ GIcNAc $\beta 1-3 G a l$ & 49 & 33 & 68 \\
\hline 17 & LewisY (LeY) pentaose & Fuc $\alpha 1-2$ Gal $\beta 1-4$ (Fuc $\alpha 1-3)$ GlcNAc $\beta 1-3 G$ al & 78 & 11 & 14 \\
\hline 18 & Lewisa (Lea) tetraose & Gal $\beta 1-3($ Fuc $\alpha 1-4)$ GIcNAc $\beta 1-3 G a l$ & 51 & 56 & 110 \\
\hline 19 & Lewisb (Leb) pentaose & Fuc $\alpha 1-2$ Gal $\beta 1-3$ (Fuc $\alpha 1-4$ )GIcNAc $\beta 1-3$ Gal & 32 & 6 & 19 \\
\hline 20 & Galili antigen pentaose & Gal $\alpha 1-3$ Gal $\beta 1-4 G|c N A c \beta 1-3 G a l \beta 1-4 G| c$ & 4 & 6 & 159 \\
\hline 21 & Isoglobopentaose / iGb5 & 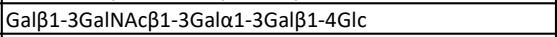 & 14 & 11 & 76 \\
\hline 22 & Galili antigen heptaose & 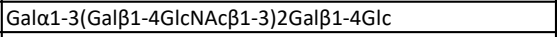 & 23 & 15 & 65 \\
\hline 23 & Globotriaose (Gb3) / Pk antigen & Gala1-4Galß1-4GIc & 19 & 16 & 84 \\
\hline 24 & Globotetraose (Gb4) / P antigen & GalNAc $\beta 1-3 G a l \alpha 1-4 G a|\beta 1-4 G| c$ & 14 & 8 & 56 \\
\hline 25 & Globo-H hexaose / Stage Specific Embryonic Antigen 3b (SSEA-3b) & Fuc $\alpha 1-2$ Gal $\beta 1-3 G a l N A c \beta 1-3 G a l \alpha 1-4 G a l \beta 1-4 G \mid c$ & -1 & 3 & -230 \\
\hline 26 & Globo-A heptaose & GalNAc $\alpha 1-3$ (Fuc $\alpha 1-2)$ Gal $\beta 1-3$ GalNAc $\beta 1-3 G a l \alpha 1-4 G a l \beta 1-4 G$ & 36 & 41 & 114 \\
\hline 27 & Globo-B heptaose & 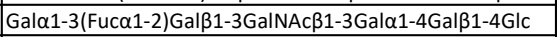 & 6 & 9 & 172 \\
\hline 28 & Blood group $\mathrm{H}$ antigen tetraose type 4 / Globo $\mathrm{H}$ tetraose & Fuc $\alpha 1-2$ Gal $\beta 1-3 G a l N A c \beta 1-3 G a l$ & 21 & 46 & 221 \\
\hline 29 & Blood group A antigen pentaose type 4 & GalNAc $\alpha 1-3$ (Fuc $\alpha 1-2)$ Gal $\beta 1-3$ GalNAc $\beta 1-3 G a l$ & 13 & 39 & 305 \\
\hline 30 & Blood group B antigen pentasaccharide type 4 & Gal $\alpha 1-3($ Fuc $\alpha 1-2)$ Gal $\beta 1-3 G a l N A c \beta 1-3 G a l$ & 3 & 6 & 254 \\
\hline 31 & Forssman antigen pentaose & GalNAc $\alpha 1-3$ GalNAc $\beta 1-3 G a l \alpha 1-4$ Gal $\beta 1-4 G \mid c$ & -8 & 3 & -44 \\
\hline 32 & Isoforssman antigen pentaose & GalNAc $\alpha 1-3 G a l N A c \beta 1-3 G a l \alpha 1-3 G a l \beta 1-4 G \mid c$ & -4 & 5 & -120 \\
\hline 33 & Globotriaose analogue type 2/ P1 antigen & 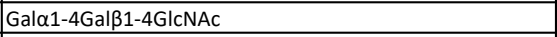 & 8 & 2 & 21 \\
\hline 34 & Isoglobo- $\mathrm{H}$ analogue type 1 & Fuc $\alpha 1-2$ Gal $\beta 1-3$ GlcNAc $\beta 1-3 G a l \alpha 1-3 G a|\beta 1-4 G| c \mid$ & 17 & 8 & 50 \\
\hline 35 & $\mathrm{OL}-05$ & GIcNAc $\beta 1-6($ GlcNAc $\beta 1-4)($ GlcNAc $\beta 1-2) M a n \alpha 1-6(G I c N A c \beta 1-$ & -5 & 117 & -2345 \\
\hline 36 & OL-09 & Man $\alpha 1-6($ Man $\alpha 1-3)$ Man $\alpha 1-6($ GlcNAc $\beta 1-4)($ GIcNAc $\beta 1-4(G I C$ & 11926 & 1594 & 13 \\
\hline 37 & OL-13 & Gal $\beta 1-4 G \mid c N A c \beta 1-2 M a n \alpha 1-6(G \mid c N A c \beta 1-4)(G a|\beta 1-4 G| c N A c \beta$ & 135 & 17 & 13 \\
\hline 38 & $\mathrm{OL}-17$ & GlcNAc $\beta 1-4($ GlcNAc $\beta 1-2)$ Man $\alpha 1-6($ GIcNAc $\beta 1-4)(G I c N A c \beta 1-$ & -3 & 4 & -136 \\
\hline 39 & EY-10 & 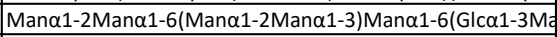 & 24611 & 1177 & 5 \\
\hline 40 & EY-15 & Man $\alpha 1-6($ Man $\alpha 1-3)$ Man $\alpha 1-6($ GlcNAc $\beta 1-4)($ GlcNAc $\beta 1-2 M a r$ & 15841 & 2742 & 17 \\
\hline 41 & EY-16 & GlcNAc $\beta 1-2 M a n \alpha 1-6($ GlcNAc $\beta 1-2 M a n \alpha 1-3) M a n \beta 1-4 G I c N A$ & 4181 & 237 & 6 \\
\hline 42 & EY-26 & GIcNAc $\beta 1-2 M a n \alpha 1-6($ GlcNAc $\beta 1-4)(G I c N A c \beta 1-2 M a n \alpha 1-3) M$ & 1579 & 79 & 5 \\
\hline 43 & EY-32 & GIcNAc $\beta 1-6($ GlcNAc $\beta 1-4)($ GlcNAc $\beta 1-2) M a n \alpha 1-6(G I c N A c \beta 1-$ & 3 & 5 & 192 \\
\hline 44 & $2^{\prime}-\mathrm{FL}$ & Fuc $\alpha 1-2$ Galß1-4Glc & 125 & 85 & 68 \\
\hline 45 & 6'-SL & Neu5A $\alpha 2-6$ Gal $\beta 1-4 G l c$ & 2 & 15 & 661 \\
\hline 46 & EY-20 & $\begin{array}{l}\text { Neu5A } \alpha 2-6 \text { Gal } \beta 1-4 G I c N A c \beta 1-2 M a n \alpha 1-6(G I c N A c \beta 1- \\
2 \text { Man } \alpha 1-3) \text { Man } \beta 1-4 G I c N A c \beta 1-4 G I c N A c\end{array}$ & 1764 & 122 & 7 \\
\hline 47 & Man8 & 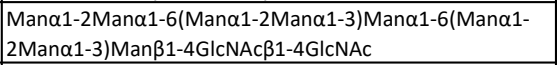 & 30258 & 814 & 3 \\
\hline 48 & FBS-1 & $\begin{array}{l}\text { Neu5A } \alpha 2-3 \text { Gal } \beta 1-4 G I c N A c \beta 1-2 M a n \alpha 1-6(N e u 5 A \alpha 2-3 G a l \beta 1- \\
\text { 4GIcNAc } \beta 1-4((\text { Neu5A } \alpha 2-3 G a l \beta 1-4 G I c N A c \beta 1-2) \text { Man } \alpha 1- \\
\text { 3)Man } \beta 1-4 G I c N A c \beta 1-4 G I c N A c\end{array}$ & -12 & 2 & -20 \\
\hline 49 & Water & & 10 & 5 & 47 \\
\hline 50 & Water & & 9 & 6 & 71 \\
\hline 51 & Water & & 1 & 8 & 800 \\
\hline 52 & Biotin-BSA & & 35045 & 9128 & 26 \\
\hline
\end{tabular}


Table S4. Printed microarray raw data. Column A to C list the ID, name and structure of printed glycans; Column D is the average relative fluorescent units (RFU); Column E is the standard deviation of 4 printed spots; Column F is the ratio between STDEV and average RFU.

\begin{tabular}{|c|c|c|c|c|c|}
\hline \multicolumn{6}{|c|}{ ConA: $0.1 \mathrm{ug} / \mathrm{mL}$} \\
\hline Glycan ID & Glycan name & Structure & Average & STDEV & $\% \mathrm{CV}$ \\
\hline 1 & Lacto-N-tetraose (LNT) & 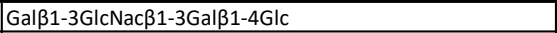 & 126 & 30 & 24 \\
\hline 2 & Lacto-N-neotetraose (LNnT / neo-LNT) & Galß1-4GIcNAcß1-3Galß1-4GIc & 84 & 48 & 57 \\
\hline 3 & Blood group $\mathrm{H}$ antigen pentaose type 2 / Lacto-N-neofucopentaose I (LnNFP I) & Fuc $\alpha 1-2$ Gal $\beta 1-4 G I c N A c \beta 1-3 G a l \beta 1-4 G l c$ & 45 & 11 & 24 \\
\hline 4 & Blood group A Lewisb antigen pentaose type 1 & GalNAc $\alpha 1-3$ (Fuc $\alpha 1-2)$ Galß1-3(Fuc $\alpha 1-4)$ GIcNAc & 52 & 29 & 56 \\
\hline 5 & Blood group A LewisY antigen pentaose type 2 & GalNAc $\alpha 1-3$ (Fuc $\alpha 1-2)$ Galß1-4(Fuc $\alpha 1-3)$ GIcNAc & 41 & 11 & 27 \\
\hline 6 & Blood group A antigen pentaose type 1 & 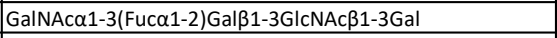 & 44 & 11 & 25 \\
\hline 7 & Blood group A antigen pentaose type 2 & GalNAc $\alpha 1-3$ (Fuc $\alpha 1-2)$ Gal $\beta 1-4$ GIcNAc $\beta 1-3 G a l$ & 32 & 10 & 32 \\
\hline 8 & Blood group $A$ antigen hexaose type 1 & GalNAc $\alpha 1-3$ (Fuc $\alpha 1-2)$ Gal $\beta 1-3 G I c N A c \beta 1-3 G a l \beta 1-4 G \mid c$ & 21 & 4 & 21 \\
\hline 9 & Blood group $A$ antigen hexaose type 2 & GalNAc $\alpha 1-3($ Fuc $\alpha 1-2)$ Gal $\beta 1-4 G I c N A c \beta 1-3 G a l \beta 1-4 G l c$ & 26 & 22 & 85 \\
\hline 10 & Blood group B Lewisb antigen pentaose type 1 & Gal $\alpha 1-3$ (Fuc $\alpha 1-2$ )Galß1-3(Fuc $\alpha 1-4)$ GlcNAc & 24 & 8 & 33 \\
\hline 11 & Blood group B LewisY antigen pentaose type 2 & Gal $\alpha 1-3$ (Fuc $\alpha 1-2)$ Galß1-4(Fuc $\alpha 1-3)$ GIcNAc & 41 & 15 & 37 \\
\hline 12 & Blood group B antigen pentaose type 1 & 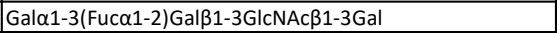 & 21 & 7 & 33 \\
\hline 13 & Blood group B antigen pentaose type 2 & 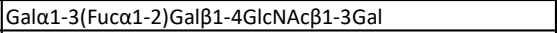 & 24 & 13 & 56 \\
\hline 14 & Blood group B antigen hexaose type 1 & Gal $\alpha 1-3$ (Fuc $\alpha 1-2)$ Gal $\beta 1-3$ GIcNAc $\beta 1-3 G a l \beta 1-4 G l c$ & 24 & 8 & 35 \\
\hline 15 & Blood group B antigen hexaose type 2 & Gal $\alpha 1-3$ (Fuc $\alpha 1-2)$ Gal $\beta 1-4$ GIcNAc $\beta 1-3 G a l \beta 1-4 G l c$ & 19 & 6 & 33 \\
\hline 16 & LewisX (LeX) tetraose & Gal $\beta 1-4$ ( Fuc $\alpha 1-3)$ GlcNAc $\beta 1-3 G a l$ & 11 & 3 & 29 \\
\hline 17 & LewisY (LeY) pentaose & 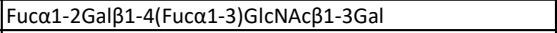 & 16 & 4 & 23 \\
\hline 18 & Lewisa (Lea) tetraose & 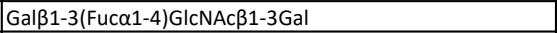 & -260 & 433 & -167 \\
\hline 19 & Lewisb (Leb) pentaose & Fuc $\alpha 1-2$ Gal $\beta 1-3$ (Fuc $\alpha 1-4$ )GlcNAc $\beta 1-3 \mathrm{Gal}$ & -2 & 55 & -2726 \\
\hline 20 & Galili antigen pentaose & 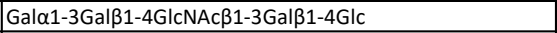 & 13 & 13 & 105 \\
\hline 21 & Isoglobopentaose / iGb5 & Galß1-3GalNAc $\beta 1-3 G a l \alpha 1-3 G a l \beta 1-4 G l c$ & 31 & 15 & 50 \\
\hline 22 & Galili antigen heptaose & 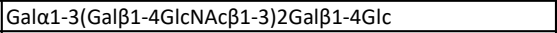 & -194 & 451 & -233 \\
\hline 23 & Globotriaose (Gb3) / Pk antigen & Gal $\alpha 1-4$ Galß1-4GIc & -9 & 58 & -627 \\
\hline 24 & Globotetraose (Gb4) / $P$ antigen & 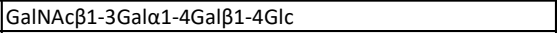 & 45 & 19 & 41 \\
\hline 25 & Globo-H hexaose / Stage Specific Embryonic Antigen 3b (SSEA-3b) & Fuca1-2Gal $\beta 1-3 G a l N A c \beta 1-3 G a l \alpha 1-4 G a l \beta 1-4 G \mid c$ & 36 & 36 & 103 \\
\hline 26 & Globo-A heptaose & GalNAc $\alpha 1-3$ (Fuc $\alpha 1-2)$ Galß1-3GalNAc $\beta 1-3 G$ Gal $\alpha 1-4$ Gal $\beta 1-4 G$ & 28 & 16 & 58 \\
\hline 27 & Globo-B heptaose & 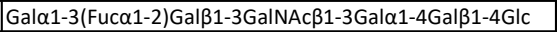 & 15 & 6 & 44 \\
\hline 28 & Blood group $\mathrm{H}$ antigen tetraose type 4 / Globo $\mathrm{H}$ tetraose & Fuc $\alpha 1-2$ Gal $\beta 1-3$ GalNAc $\beta 1-3 \mathrm{Gal}$ & 7 & 3 & 44 \\
\hline 29 & Blood group $\mathrm{A}$ antigen pentaose type 4 & 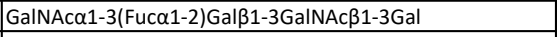 & 18 & 6 & 33 \\
\hline 30 & Blood group B antigen pentasaccharide type 4 & 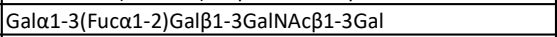 & 18 & 7 & 40 \\
\hline 31 & Forssman antigen pentaose & GalNAc $\alpha 1-3 G a l N A c \beta 1-3 G a l \alpha 1-4 G a l \beta 1-4 G \mid c$ & 10 & 5 & 49 \\
\hline 32 & Isoforssman antigen pentaose & GaINAc $\alpha 1-3$ GalNAc $\beta 1-3$ Gal $\alpha 1-3$ Gal $\beta 1-4 G I c$ & 28 & 15 & 56 \\
\hline 33 & Globotriaose analogue type 2/ P1 antigen & Gal $\alpha 1-4$ Galß1-4GIcNAc & 8 & 7 & 88 \\
\hline 34 & Isoglobo- $\mathrm{H}$ analogue type 1 & Fuc $\alpha 1-2$ Gal $\beta 1-3 G I c N A c \beta 1-3 G a l \alpha 1-3 G a \mid \beta 1-4 G I c$ & 12 & 3 & 23 \\
\hline 35 & OL-05 & GlcNAc $\beta 1-6(G \mid c N A c \beta 1-4)(G \mid c N A c \beta 1-2) M a n \alpha 1-6(G \mid c N A c \beta 1-$ & 4776 & 165 & 3 \\
\hline 36 & OL-09 & 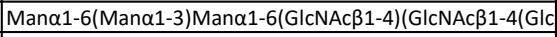 & 58516 & 1545 & 3 \\
\hline 37 & OL-13 & Gal $\beta 1-4 G \mid c N A c \beta 1-2 M a n \alpha 1-6(G \mid c N A c \beta 1-4)(G a l \beta 1-4 G \mid c N A c \beta$ & 11195 & 391 & 3 \\
\hline 38 & OL-17 & GIcNAc $\beta 1-4($ GlcNAc $\beta 1-2)$ Man $\alpha 1-6($ GIcNAc $\beta 1-4)(G I c N A c \beta 1-$ & 53 & 8 & 15 \\
\hline 39 & EY-10 & 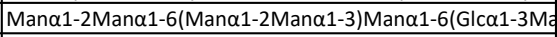 & 59715 & 1414 & 2 \\
\hline 40 & EY-15 & Man $\alpha 1-6($ Man $\alpha 1-3)$ Man $\alpha 1-6($ GlcNAc $\beta 1-4)($ GlcNAc $\beta 1-2 M a r$ & 59610 & 2273 & 4 \\
\hline 41 & EY-16 & GlcNAc $\beta 1-2 M a n \alpha 1-6($ GlcNAc $\beta 1-2 M a n \alpha 1-3) M a n \beta 1-4 G I c N A$ & 43063 & 3155 & 7 \\
\hline 42 & EY-26 & GIcNAc $\beta 1-2 M a n \alpha 1-6(G I c N A c \beta 1-4)(G I c N A c \beta 1-2 M a n \alpha 1-3) M$ & 23652 & 803 & 3 \\
\hline 43 & EY-32 & GlcNAc $\beta 1-6($ GlcNAc $\beta 1-4)($ GlcNAc $\beta 1-2) M a n \alpha 1-6(G \mid c N A c \beta 1-$ & 251 & 18 & 7 \\
\hline 44 & 2'-FL & Fuc $\alpha 1-2 \mathrm{Gal} \beta 1-4 \mathrm{Glc}$ & 10 & 4 & 40 \\
\hline 45 & 6'-SL & Neu5A $\alpha 2-6$ Gal $\beta 1-4 G I c$ & 5 & 2 & 33 \\
\hline 46 & EY-20 & 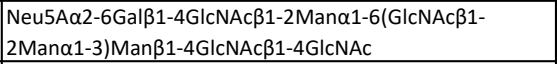 & 20383 & 961 & 5 \\
\hline 47 & Man8 & 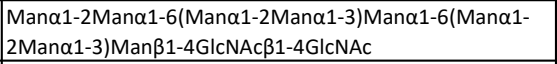 & 52896 & 1304 & 2 \\
\hline 48 & FBS-1 & $\begin{array}{l}\text { Neu5A } \alpha 2-3 G a l \beta 1-4 G I c N A c \beta 1-2 \text { Man } \alpha 1-6(\text { Neu5A } \alpha 2-3 G a l \beta 1- \\
\text { 4GIcNAc } \beta 1-4((\text { Neu5A } \alpha 2-3 G a l \beta 1-4 G I c N A c \beta 1-2) \text { Man } \alpha 1- \\
\text { 3)Man } \beta 1-4 G I c N A c \beta 1-4 G I c N A c\end{array}$ & 164 & 368 & 224 \\
\hline 49 & Water & & 11 & 4 & 32 \\
\hline 50 & Water & & 25 & 20 & 79 \\
\hline 51 & Water & & 13 & 1 & 9 \\
\hline 52 & Biotin-BSA & & 19185 & 2269 & 12 \\
\hline
\end{tabular}


Table S4. Printed microarray raw data. Column A to C list the ID, name and structure of printed glycans; Column D is the average relative fluorescent units (RFU); Column E is the standard deviation of 4 printed spots; Column $\mathrm{F}$ is the ratio between STDEV and average RFU.

\begin{tabular}{|c|c|c|c|c|c|}
\hline \multicolumn{6}{|c|}{ ConA: $1 \mathrm{ug} / \mathrm{mL}$} \\
\hline Glycan ID & Glycan name & Structure & Average & STDEV & $\% \mathrm{CV}$ \\
\hline 1 & Lacto-N-tetraose (LNT) & Galß1-3GIcNacß1-3Galß1-4GIc & 389 & 125 & 32 \\
\hline 2 & Lacto-N-neotetraose (LNnT / neo-LNT) & Galß1-4GIcNAcß1-3Galß1-4GIc & 204 & 33 & 16 \\
\hline 3 & Blood group $\mathrm{H}$ antigen pentaose type 2 / Lacto-N-neofucopentaose I (LnNFP I) & Fuc $\alpha 1-2$ Gal $\beta 1-4 G I c N A c \beta 1-3 G a \mid \beta 1-4 G I c$ & 119 & 29 & 24 \\
\hline 4 & Blood group A Lewisb antigen pentaose type 1 & GalNAc $\alpha 1-3$ (Fuc $\alpha 1-2$ )Galß1-3(Fuc $\alpha 1-4)$ GIcNAc & 155 & 10 & 7 \\
\hline 5 & Blood group A LewisY antigen pentaose type 2 & GalNAc $\alpha 1-3$ (Fuc $\alpha 1-2$ )Gal $\beta 1-4($ Fuc $\alpha 1-3)$ GIcNAc & 148 & 12 & 8 \\
\hline 6 & Blood group $A$ antigen pentaose type 1 & 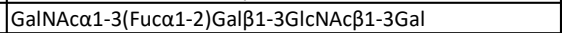 & 93 & 44 & 48 \\
\hline 7 & Blood group $A$ antigen pentaose type 2 & GalNAca1-3(Fuc $\alpha 1-2)$ Gal $\beta 1-4 G I c N A c \beta 1-3 G a l$ & 156 & 10 & 7 \\
\hline 8 & Blood group $\mathrm{A}$ antigen hexaose type 1 & GalNAc $\alpha 1-3$ (Fuc $\alpha 1-2)$ Gal $\beta 1-3$ GIcNAc $\beta 1-3 G a l \beta 1-4 G \mid c$ & 101 & 13 & 13 \\
\hline 9 & Blood group $\mathrm{A}$ antigen hexaose type 2 & GalNAc $\alpha 1-3$ (Fuc $\alpha 1-2)$ Gal $\beta 1-4 G I c N A c \beta 1-3 G a l \beta 1-4 G I c$ & 39 & 10 & 27 \\
\hline 10 & Blood group B Lewisb antigen pentaose type 1 & Gal $\alpha 1-3$ (Fuc $\alpha 1-2$ )Galß1-3(Fuc $\alpha 1-4)$ GIcNAc & 52 & 14 & 27 \\
\hline 11 & Blood group B LewisY antigen pentaose type 2 & Gal $\alpha 1-3$ (Fuc $\alpha 1-2$ )Galß1-4(Fuc $\alpha 1-3)$ GIcNAc & 51 & 18 & 35 \\
\hline 12 & Blood group B antigen pentaose type 1 & 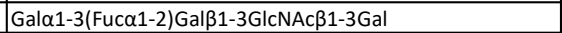 & 80 & 21 & 26 \\
\hline 13 & Blood group B antigen pentaose type 2 & Gal $\alpha 1-3$ (Fuc $\alpha 1-2$ )Gal $\beta 1-4 G I c N A c \beta 1-3 G a l$ & 51 & 70 & 138 \\
\hline 14 & Blood group B antigen hexaose type 1 & Gal $\alpha 1-3$ (Fuc $\alpha 1-2)$ Gal $\beta 1-3 G|c N A c \beta 1-3 G a| \beta 1-4 G \mid c$ & 127 & 37 & 29 \\
\hline 15 & Blood group B antigen hexaose type 2 & Gal $\alpha 1-3$ (Fuc $\alpha 1-2)$ Gal $\beta 1-4 G|c N A c \beta 1-3 G a l \beta 1-4 G| c$ & 85 & 28 & 32 \\
\hline 16 & LewisX (LeX) tetraose & Gal $\beta 1-4$ ( Fuc $\alpha 1-3$ )GIcNAc $\beta 1-3 \mathrm{Gal}$ & 89 & 24 & 27 \\
\hline 17 & LewisY (LeY) pentaose & 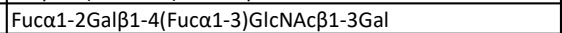 & 44 & 31 & 71 \\
\hline 18 & Lewisa (Lea) tetraose & Gal $\beta 1-3$ (Fuc $\alpha 1-4)$ GIcNAc $\beta 1-3 G a l$ & 94 & 33 & 35 \\
\hline 19 & Lewisb (Leb) pentaose & 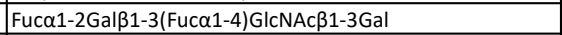 & 183 & 167 & 92 \\
\hline 20 & Galili antigen pentaose & Gal $\alpha 1-3$ Gal $\beta 1-4 G I c N A c \beta 1-3 G a l \beta 1-4 G l c$ & 78 & 39 & 49 \\
\hline 21 & Isoglobopentaose / iGb5 & 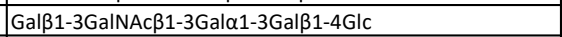 & 140 & 65 & 46 \\
\hline 22 & Galili antigen heptaose & Gal $\alpha 1-3($ Gal$\beta 1-4 G \mid c N A c \beta 1-3) 2 G a|\beta 1-4 G| c$ & 115 & 23 & 20 \\
\hline 23 & Globotriaose (Gb3) / Pk antigen & Gala1-4Galß1-4GIc & 135 & 28 & 20 \\
\hline 24 & Globotetraose (Gb4) / P antigen & GalNAc $\beta 1-3 G$ Gal $\alpha 1-4$ Gal $\beta 1-4 G \mid c$ & 168 & 25 & 15 \\
\hline 25 & Globo-H hexaose / Stage Specific Embryonic Antigen 3b (SSEA-3b) & Fuc $\alpha 1-2$ Gal $\beta 1-3$ GalNAc $\beta 1-3 G a l \alpha 1-4 G a l \beta 1-4 G I c$ & 80 & 12 & 16 \\
\hline 26 & Globo-A heptaose & 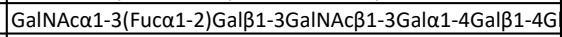 & 80 & 32 & 40 \\
\hline 27 & Globo-B heptaose & Gal $\alpha 1-3$ (Fuc $\alpha 1-2)$ Gal $\beta 1-3 G$ GalNAc $\beta 1-3 G a l \alpha 1-4 G a l \beta 1-4 G l c$ & 64 & 17 & 27 \\
\hline 28 & Blood group $\mathrm{H}$ antigen tetraose type 4 / Globo $\mathrm{H}$ tetraose & Fuc $\alpha 1-2$ Gal $\beta 1-3 G a l N A c \beta 1-3 G a l$ & 69 & 11 & 16 \\
\hline 29 & Blood group $A$ antigen pentaose type 4 & GalNAca1-3(Fuc $\alpha 1-2)$ Galß1-3GalNAc $\beta 1-3 G a l$ & 136 & 47 & 35 \\
\hline 30 & Blood group B antigen pentasaccharide type 4 & Gal $\alpha 1-3$ (Fuc $\alpha 1-2$ )Gal $\beta 1-3 G a l N A c \beta 1-3 G a l$ & 91 & 4 & 5 \\
\hline 31 & Forssman antigen pentaose & GaINAc $\alpha 1-3$ GaINAc $\beta 1-3 G a l \alpha 1-4 G a l \beta 1-4 G I c$ & 74 & 34 & 47 \\
\hline 32 & Isoforssman antigen pentaose & GaINAc $\alpha 1-3$ GalNAc $\beta 1-3 G a l \alpha 1-3 G a l \beta 1-4 G I c$ & 58 & 25 & 43 \\
\hline 33 & Globotriaose analogue type 2/ P1 antigen & Gal 1 1-4Galß1-4GIcNAc & 119 & 24 & 20 \\
\hline 34 & Isoglobo- $\mathrm{H}$ analogue type 1 & Fuc $\alpha 1-2$ Gal $\beta 1-3$ GIcNAc $\beta 1-3 G a l \alpha 1-3 G a l \beta 1-4 G \mid c$ & 68 & 55 & 80 \\
\hline 35 & OL-05 & GIcNAc $\beta 1-6(G I c N A c \beta 1-4)(G \mid c N A c \beta 1-2) M a n \alpha 1-6(G \mid c N A c \beta 1-$ & 52794 & 1648 & 3 \\
\hline 36 & OL-09 & Man $\alpha 1-6($ Man $\alpha 1-3)$ Man $\alpha 1-6($ GIcNAc $\beta 1-4)(G I c N A c \beta 1-4$ (GIC & 61682 & 1130 & 2 \\
\hline 37 & OL-13 & 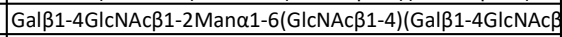 & 63000 & 692 & 1 \\
\hline 38 & OL-17 & GIcNAc $\beta 1-4($ GlcNAc $\beta 1-2)$ Man $\alpha 1-6($ GlcNAc $\beta 1-4)(G I c N A c \beta 1-$ & 2202 & 678 & 31 \\
\hline 39 & EY-10 & 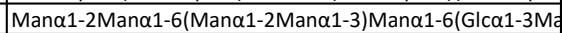 & 58892 & 386 & 1 \\
\hline 40 & EY-15 & Man $\alpha 1-6($ Man $\alpha 1-3)$ Man $\alpha 1-6($ GIcNAc $\beta 1-4)(G I c N A c \beta 1-2 M a r$ & 63387 & 1839 & 3 \\
\hline 41 & EY-16 & GIcNAc $\beta 1-2 M a n \alpha 1-6(G I c N A c \beta 1-2 M a n \alpha 1-3)$ Man $\beta 1-4 G I c N A$ & 59955 & 1231 & 2 \\
\hline 42 & EY-26 & GlcNAc $\beta 1-2 M a n \alpha 1-6(G l c N A c \beta 1-4)(G I c N A c \beta 1-2 M a n \alpha 1-3) M$ & 62068 & 1584 & 3 \\
\hline 43 & EY-32 & GlcNAc $\beta 1-6($ GIcNAc $\beta 1-4)($ GlcNAc $\beta 1-2) M a n \alpha 1-6($ GlcNAc $\beta 1-$ & 5372 & 414 & 8 \\
\hline 44 & $2 '-F L$ & Fuc $\alpha 1-2$ Gal $\beta 1-4 G I c$ & 115 & 20 & 17 \\
\hline 45 & 6'-SL & Neu5A $\alpha 2-6$ Gal $\beta 1-4 G l c$ & 64 & 49 & 77 \\
\hline 46 & EY-20 & $\begin{array}{l}\text { Neu5A } \alpha 2-6 \text { Gal } \beta 1-4 G I c N A c \beta 1-2 \text { Man } \alpha 1-6 \text { (GIcNAc } \beta 1- \\
\text { 2Man } \alpha 1-3) \text { Man } \beta 1-4 G I c N A c \beta 1-4 G I c N A c\end{array}$ & 57220 & 4327 & 8 \\
\hline 47 & Man8 & 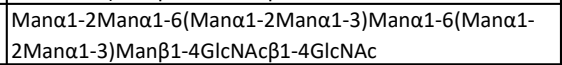 & 57383 & 1464 & 3 \\
\hline 48 & FBS-1 & 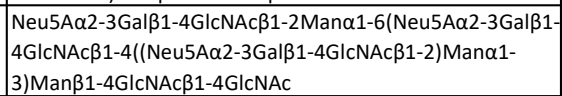 & 120 & 39 & 32 \\
\hline 49 & Water & & 164 & 56 & 34 \\
\hline 50 & Water & & 164 & 60 & 37 \\
\hline 51 & Water & & 162 & 44 & 28 \\
\hline 52 & Biotin-BSA & & 18612 & 2984 & 16 \\
\hline
\end{tabular}


Table S4. Printed microarray raw data. Column A to C list the ID, name and structure of printed glycans; Column D is the average relative fluorescent units (RFU); Column E is the standard deviation of 4 printed spots; Column $\mathrm{F}$ is the ratio between STDEV and average RFU.

\begin{tabular}{|c|c|c|c|c|c|}
\hline \multicolumn{6}{|c|}{ AAL: $0.001 \mathrm{ug} / \mathrm{mL}$} \\
\hline Glycan ID & Glycan name & Structure & Average & STDEV & $\% \mathrm{CV}$ \\
\hline 1 & Lacto-N-tetraose (LNT) & Galß1-3GIcNac $\beta 1-3 G a l \beta 1-4 G \mid c$ & 157 & 47 & 30 \\
\hline 2 & Lacto-N-neotetraose (LNnT / neo-LNT) & Galß1-4GIcNAcß1-3Galß1-4GIc & 52 & 17 & 33 \\
\hline 3 & Blood group $\mathrm{H}$ antigen pentaose type 2 / Lacto-N-neofucopentaose I (LnNFP I) & Fuc $\alpha 1-2$ Galß1-4GIcNAc $\beta 1-3 G a \mid \beta 1-4 G I c$ & 8206 & 381 & 5 \\
\hline 4 & Blood group A Lewisb antigen pentaose type 1 & GalNAc $\alpha 1-3($ Fuc $\alpha 1-2)$ Gal $\beta 1-3($ Fuc $\alpha 1-4)$ GlcNAc & 6304 & 305 & 5 \\
\hline 5 & Blood group A LewisY antigen pentaose type 2 & GalNAc $\alpha 1-3$ (Fuc $\alpha 1-2)$ Gal $\beta 1-4$ (Fuc $\alpha 1-3)$ GIcNAc & 1582 & 55 & 3 \\
\hline 6 & Blood group $\mathrm{A}$ antigen pentaose type 1 & 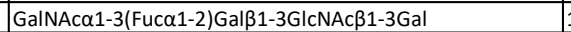 & 18 & 12 & 69 \\
\hline 7 & Blood group $\mathrm{A}$ antigen pentaose type 2 & GalNAc $\alpha 1-3$ (Fuc $\alpha 1-2)$ Gal $\beta 1-4 G I c N A c \beta 1-3 G a l$ & 11 & 4 & 39 \\
\hline 8 & Blood group $A$ antigen hexaose type 1 & GalNAc $\alpha 1-3$ (Fuc $\alpha 1-2)$ Gal $\beta 1-3 G I c N A c \beta 1-3 G a l \beta 1-4 G I c$ & 28 & 45 & 164 \\
\hline 9 & Blood group $A$ antigen hexaose type 2 & GalNAc $\alpha 1-3$ (Fuc $\alpha 1-2)$ Gal $\beta 1-4 G I c N A c \beta 1-3 G a l \beta 1-4 G I c$ & 29 & 33 & 113 \\
\hline 10 & Blood group B Lewisb antigen pentaose type 1 & Gal $\alpha 1-3$ (Fuc $\alpha 1-2)$ Gal $\beta 1-3$ (Fuc $\alpha 1-4)$ GIcNAc & 3430 & 157 & 5 \\
\hline 11 & Blood group B LewisY antigen pentaose type 2 & 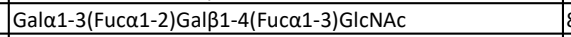 & 8038 & 352 & 4 \\
\hline 12 & Blood group $\mathrm{B}$ antigen pentaose type 1 & Gal $\alpha 1-3$ (Fuc $\alpha 1-2$ )Gal $\beta 1-3$ GIcNAc $\beta 1-3 G a l$ & 3 & 4 & 127 \\
\hline 13 & Blood group B antigen pentaose type 2 & Gal $\alpha 1-3$ (Fuc $\alpha 1-2)$ Gal $\beta 1-4 G I c N A c \beta 1-3 G a l$ & 3 & 3 & 100 \\
\hline 14 & Blood group B antigen hexaose type 1 & Gal $\alpha 1-3$ (Fuc $\alpha 1-2)$ Gal $\beta 1-3 G|c N A c \beta 1-3 G a l \beta 1-4 G| c$ & 1 & 5 & 959 \\
\hline 15 & Blood group B antigen hexaose type 2 & Gal $\alpha 1-3($ Fuc $\alpha 1-2)$ Gal $\beta 1-4$ GIcNAc $\beta 1-3 G a l \beta 1-4 G I c$ & -2 & 2 & -92 \\
\hline 16 & LewisX (LeX) tetraose & Gal $\beta 1-4$ ( Fuc $\alpha 1-3$ )GIcNAc $\beta 1-3 \mathrm{Gal}$ & 4408 & 828 & 19 \\
\hline 17 & LewisY (LeY) pentaose & 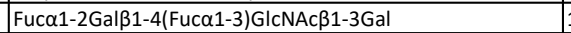 & 15886 & 973 & 6 \\
\hline 18 & Lewisa (Lea) tetraose & Galß1-3(Fuc $\alpha 1-4)$ GIcNAc $\beta 1-3 G a l$ & 8131 & 397 & 5 \\
\hline 19 & Lewisb (Leb) pentaose & Fuc $\alpha 1-2$ Gal $\beta 1-3$ (Fuc $\alpha 1-4)$ GIcNAc $\beta 1-3 G a l$ & 3352 & 403 & 12 \\
\hline 20 & Galili antigen pentaose & Gal $\alpha 1-3$ Gal $\beta 1-4 G I c N A c \beta 1-3 G a l \beta 1-4 G I c$ & -1 & 4 & -702 \\
\hline 21 & Isoglobopentaose / iGb5 & 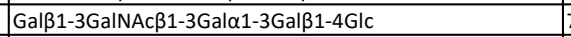 & 7 & 1 & 14 \\
\hline 22 & Galili antigen heptaose & Gal $\alpha 1-3($ Gal$\beta 1-4 G I c N A c \beta 1-3) 2$ Galß1-4GIc & 1 & 3 & 316 \\
\hline 23 & Globotriaose (Gb3) / Pk antigen & Gal $\alpha 1-4 \mathrm{Gal} \beta 1-4 \mathrm{Glc}$ & 7 & 1 & 20 \\
\hline 24 & Globotetraose (Gb4) / P antigen & 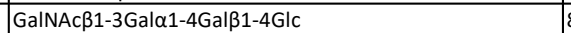 & 8 & 4 & 52 \\
\hline 25 & Globo-H hexaose / Stage Specific Embryonic Antigen 3b (SSEA-3b) & Fuc $\alpha 1-2$ Gal $\beta 1-3 G$ alNAc $\beta 1-3 G a l \alpha 1-4 G a l \beta 1-4 G l c$ & 4 & 11 & 317 \\
\hline 26 & Globo-A heptaose & GalNAc $\alpha 1-3$ (Fuc $\alpha 1-2)$ Gal $\beta 1-3 G$ alNAc $\beta 1-3 G a l \alpha 1-4 G a l \beta 1-4 G$ & -1 & 5 & -420 \\
\hline 27 & Globo-B heptaose & Gal $\alpha 1-3$ (Fuc $\alpha 1-2)$ Gal $\beta 1-3 G a l N A c \beta 1-3 G a l \alpha 1-4 G a l \beta 1-4 G \mid c$ & -14 & 16 & -111 \\
\hline 28 & Blood group $\mathrm{H}$ antigen tetraose type 4 / Globo $\mathrm{H}$ tetraose & Fuc $\alpha 1-2$ Gal $\beta 1-3 G$ alNAc $\beta 1-3 G a l$ & 41 & 4 & 9 \\
\hline 29 & Blood group $\mathrm{A}$ antigen pentaose type 4 & 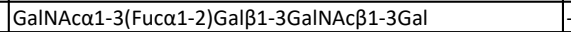 & -5 & 5 & -96 \\
\hline 30 & Blood group B antigen pentasaccharide type 4 & Gal $\alpha 1-3$ (Fuc $\alpha 1-2$ )Gal $\beta 1-3$ GalNAc $\beta 1-3 \mathrm{Gal}$ & -4 & 6 & -164 \\
\hline 31 & Forssman antigen pentaose & GalNAc $\alpha 1-3$ GalNAc $\beta 1-3$ Gal $\alpha 1-4$ Gal $\beta 1-4 G l c$ & -12 & 10 & -89 \\
\hline 32 & Isoforssman antigen pentaose & GalNAc $\alpha 1-3 G a l N A c \beta 1-3 G a l \alpha 1-3 G a l \beta 1-4 G \mid c$ & 3 & 4 & 139 \\
\hline 33 & Globotriaose analogue type 2/ P1 antigen & Gal $\alpha 1-4$ Galß1-4GIcNAc & 9 & 6 & 69 \\
\hline 34 & Isoglobo- $\mathrm{H}$ analogue type 1 & 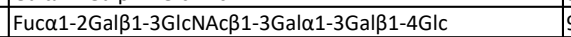 & 9626 & 521 & 5 \\
\hline 35 & OL-05 & GIcNAc $\beta 1-6(G I c N A c \beta 1-4)(G I c N A c \beta 1-2)$ Man $\alpha 1-6(G I c N A c \beta 1-$ & -2 & 4 & -187 \\
\hline 36 & OL-09 & Man $\alpha 1-6($ Man $\alpha 1-3)$ Man $\alpha 1-6($ GIcNAc $\beta 1-4)(G I c N A c \beta 1-4(G I C$ & 3 & 5 & 186 \\
\hline 37 & OL-13 & Gal $\beta 1-4$ GIcNAc $\beta 1-2 M a n \alpha 1-6(G I c N A c \beta 1-4)(G a l \beta 1-4 G I c N A c \beta$ & 6 & 23 & 376 \\
\hline 38 & OL-17 & GIcNAc $\beta 1-4(G I c N A c \beta 1-2) M a n \alpha 1-6(G I c N A c \beta 1-4)(G I c N A c \beta 1-$ & -2 & 3 & -135 \\
\hline 39 & EY-10 & Man $\alpha 1-2$ Man $\alpha 1-6($ Man $\alpha 1-2 M a n \alpha 1-3)$ Man $\alpha 1-6(G I c \alpha 1-3 M A$ & 6 & 3 & 48 \\
\hline 40 & EY-15 & Man $\alpha 1-6($ Man $\alpha 1-3)$ Man $\alpha 1-6($ GIcNAc $\beta 1-4)(G I c N A c \beta 1-2 M a r$ & 3 & 7 & 249 \\
\hline 41 & EY-16 & GIcNAc $\beta 1-2 M a n \alpha 1-6(G I c N A c \beta 1-2 M a n \alpha 1-3)$ Man $\beta 1-4 G I c N A$ & -53 & 76 & -144 \\
\hline 42 & EY-26 & GlcNAc $\beta 1-2 M a n \alpha 1-6(G I c N A c \beta 1-4)(G I c N A c \beta 1-2 M a n \alpha 1-3) M$ & 16893 & 1074 & 6 \\
\hline 43 & EY-32 & GIcNAc $\beta 1-6(G \mid c N A c \beta 1-4)(G \mid c N A c \beta 1-2)$ Man $\alpha 1-6($ GIcNAc $\beta 1-$ & 3 & 2 & 81 \\
\hline 44 & $2^{\prime}-\mathrm{FL}$ & Fuc $\alpha 1-2$ Galß1-4Glc & 9208 & 2622 & 28 \\
\hline 45 & 6'-SL & Neu5A $\alpha 2-6 \mathrm{Gal} \beta 1-4 \mathrm{Glc}$ & -56 & 64 & -116 \\
\hline 46 & EY-20 & 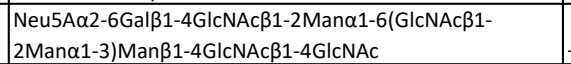 & -22 & 22 & -99 \\
\hline 47 & Man8 & 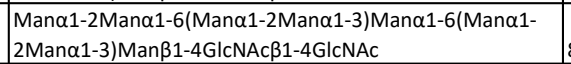 & 8 & 6 & 82 \\
\hline 48 & FBS-1 & 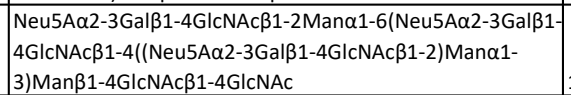 & 1 & 5 & 600 \\
\hline 49 & Water & & -6 & 3 & -48 \\
\hline 50 & Water & & -2 & 3 & -111 \\
\hline 51 & Water & & -3 & 3 & -81 \\
\hline 52 & Biotin-BSA & & 13435 & 3461 & 26 \\
\hline
\end{tabular}


Table S4. Printed microarray raw data. Column A to C list the ID, name and structure of printed glycans; Column D is the average relative fluorescent units (RFU); Column E is the standard deviation of 4 printed spots; Column $\mathrm{F}$ is the ratio between STDEV and average RFU.

\begin{tabular}{|c|c|c|c|c|c|}
\hline \multicolumn{6}{|c|}{ AAL: $0.01 \mathrm{ug} / \mathrm{mL}$} \\
\hline Glycan ID & Glycan name & Structure & Average & STDEV & $\% \mathrm{CV}$ \\
\hline 1 & Lacto-N-tetraose (LNT) & Gal $\beta 1-3 G|c N a c \beta 1-3 G a| \beta 1-4 G l c$ & 677 & 210 & 31 \\
\hline 2 & Lacto-N-neotetraose (LNnT / neo-LNT) & Galß1-4GIcNAcß1-3Galß1-4GIc & 115 & 19 & 16 \\
\hline 3 & Blood group $\mathrm{H}$ antigen pentaose type 2 / Lacto-N-neofucopentaose I (LnNFP I) & Fuc $\alpha 1-2$ Gal $\beta 1-4 G I c N A c \beta 1-3 G a l \beta 1-4 G I c$ & 61073 & 958 & 2 \\
\hline 4 & Blood group A Lewisb antigen pentaose type 1 & GalNAc $\alpha 1-3$ (Fuc $\alpha 1-2$ )Galß1-3(Fuc $\alpha 1-4)$ GIcNAc & 58526 & 2916 & 5 \\
\hline 5 & Blood group A LewisY antigen pentaose type 2 & GaINAc $\alpha 1-3$ (Fuc $\alpha 1-2$ )Galß1-4(Fuc $\alpha 1-3)$ GIcNAc & 35323 & 2726 & 8 \\
\hline 6 & Blood group $\mathrm{A}$ antigen pentaose type 1 & 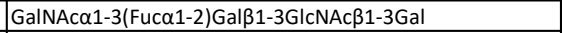 & 29 & 6 & 22 \\
\hline 7 & Blood group $\mathrm{A}$ antigen pentaose type 2 & 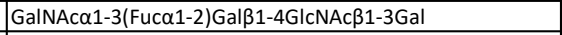 & 16 & 2 & 14 \\
\hline 8 & Blood group $A$ antigen hexaose type 1 & GalNAc $\alpha 1-3$ (Fuc $\alpha 1-2)$ Gal $\beta 1-3$ GIcNAc $\beta 1-3 G a l \beta 1-4 G l c$ & 20 & 5 & 23 \\
\hline 9 & Blood group $\mathrm{A}$ antigen hexaose type 2 & GalNAc $\alpha 1-3$ (Fuc $\alpha 1-2)$ Gal $\beta 1-4 G I c N A c \beta 1-3 G a l \beta 1-4 G l c$ & 2 & 3 & 111 \\
\hline 10 & Blood group B Lewisb antigen pentaose type 1 & Gal $\alpha 1-3$ (Fuc $\alpha 1-2$ )Gal $\beta 1-3$ (Fuc $\alpha 1-4)$ GlcNAc & 56304 & 963 & 2 \\
\hline 11 & Blood group B LewisY antigen pentaose type 2 & Gal $\alpha 1-3$ (Fuc $\alpha 1-2$ )Galß1-4(Fuc $\alpha 1-3)$ GlcNAc & 55758 & 632 & 1 \\
\hline 12 & Blood group B antigen pentaose type 1 & Gal $\alpha 1-3$ (Fuc $\alpha 1-2$ )Galß1-3GIcNAc $\beta 1-3 G a l$ & -114 & 135 & -119 \\
\hline 13 & Blood group B antigen pentaose type 2 & Gal $\alpha 1-3$ (Fuc $\alpha 1-2)$ Gal $\beta 1-4 G \mid c N A c \beta 1-3 G a l$ & 46 & 86 & 188 \\
\hline 14 & Blood group B antigen hexaose type 1 & Gal $\alpha 1-3$ (Fuc $\alpha 1-2)$ Gal $\beta 1-3$ GlcNAc $\beta 1-3 G a l \beta 1-4 G \mid c$ & 15 & 11 & 74 \\
\hline 15 & Blood group B antigen hexaose type 2 & Gal $\alpha 1-3$ (Fuc $\alpha 1-2)$ Galß1-4GIcNAc $\beta 1-3 G a l \beta 1-4 G l c$ & 10 & 4 & 40 \\
\hline 16 & LewisX (LeX) tetraose & Gal $\beta 1-4$ ( Fuc $\alpha 1-3)$ GIcNAc $\beta 1-3 G a l$ & 54241 & 6074 & 11 \\
\hline 17 & LewisY (LeY) pentaose & Fuc $\alpha 1-2$ Gal $\beta 1-4$ (Fuc $\alpha 1-3)$ GIcNAc $\beta 1-3 G a l$ & 55466 & 2342 & 4 \\
\hline 18 & Lewisa (Lea) tetraose & Galß1-3(Fuc $\alpha 1-4)$ GlcNAc $\beta 1-3 G a l$ & 58722 & 2129 & 4 \\
\hline 19 & Lewisb (Leb) pentaose & Fuc $\alpha 1-2$ Gal $\beta 1-3$ (Fuc $\alpha 1-4)$ GlcNAc $\beta 1-3 G a l$ & 57423 & 960 & 2 \\
\hline 20 & Galili antigen pentaose & Gal $\alpha 1-3$ Gal $\beta 1-4 G I c N A c \beta 1-3 G a l \beta 1-4 G l c$ & -14 & 43 & -302 \\
\hline 21 & Isoglobopentaose / iGb5 & 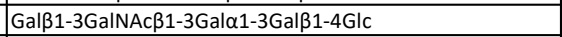 & 17 & 6 & 34 \\
\hline 22 & Galili antigen heptaose & Gal $\alpha 1-3($ Gal$\beta 1-4 G I c N A c \beta 1-3) 2 G a l \beta 1-4 G \mid c$ & 25 & 14 & 58 \\
\hline 23 & Globotriaose (Gb3) / Pk antigen & 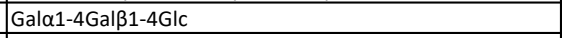 & 8 & 6 & 78 \\
\hline 24 & Globotetraose $(\mathrm{Gb} 4)$ / $P$ antigen & GalNAc $\beta 1-3$ Gal $\alpha 1-4 \mathrm{Gal} \beta 1-4 \mathrm{Glc}$ & -100 & 122 & -121 \\
\hline 25 & Globo-H hexaose / Stage Specific Embryonic Antigen 3b (SSEA-3b) & Fuc $\alpha 1-2$ Gal $\beta 1-3 G a l N A c \beta 1-3 G a l \alpha 1-4 G a l \beta 1-4 G l c$ & 103 & 18 & 18 \\
\hline 26 & Globo-A heptaose & 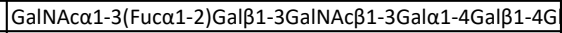 & 8 & 7 & 82 \\
\hline 27 & Globo-B heptaose & Gal $\alpha 1-3($ Fuc $\alpha 1-2)$ Galß1-3GalNAc $\beta 1-3 G a l \alpha 1-4 G a l \beta 1-4 G l c$ & 1 & 5 & 1039 \\
\hline 28 & Blood group $\mathrm{H}$ antigen tetraose type 4 / Globo $\mathrm{H}$ tetraose & Fuc $\alpha 1-2$ Galß1-3GalNAc $\beta 1-3 G a l$ & 10832 & 2465 & 23 \\
\hline 29 & Blood group $\mathrm{A}$ antigen pentaose type 4 & 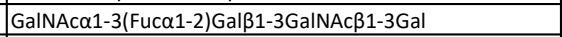 & 4 & 7 & 198 \\
\hline 30 & Blood group B antigen pentasaccharide type 4 & Gal $\alpha 1-3$ (Fuc $\alpha 1-2)$ Gal $\beta 1-3 G a l N A c \beta 1-3 G a l$ & -40 & 46 & -114 \\
\hline 31 & Forssman antigen pentaose & GalNAc $\alpha 1-3$ GalNAc $\beta 1-3 G a l \alpha 1-4 G a l \beta 1-4 G I c$ & -1 & 2 & -183 \\
\hline 32 & Isoforssman antigen pentaose & GalNAc $\alpha 1-3$ GalNAc $\beta 1-3 G a l \alpha 1-3 G a l \beta 1-4 G l c$ & 23 & 52 & 226 \\
\hline 33 & Globotriaose analogue type 2/ P1 antigen & 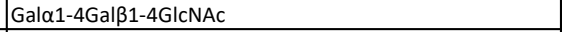 & 50 & 18 & 36 \\
\hline 34 & Isoglobo- $\mathrm{H}$ analogue type 1 & Fuc $\alpha 1-2$ Gal $\beta 1-3 G I c N A c \beta 1-3 G a l \alpha 1-3 G a l \beta 1-4 G l c$ & 59460 & 3899 & 7 \\
\hline 35 & OL-05 & GlcNAc $\beta 1-6(G \mid c N A c \beta 1-4)(G \mid c N A c \beta 1-2) M a n \alpha 1-6(G \mid c N A c \beta 1-$ & 13 & 17 & 132 \\
\hline 36 & OL-09 & Man $\alpha 1-6($ Man $\alpha 1-3)$ Man $\alpha 1-6($ GlcNAc $\beta 1-4)(G I c N A c \beta 1-4(G I C$ & -2 & 3 & -221 \\
\hline 37 & OL-13 & 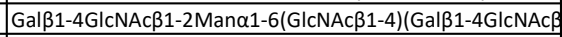 & -4 & 1 & -35 \\
\hline 38 & OL-17 & GlcNAc $\beta 1-4(G I c N A c \beta 1-2) M a n \alpha 1-6(G l c N A c \beta 1-4)(G I c N A c \beta 1-$ & -18 & 28 & -153 \\
\hline 39 & EY-10 & 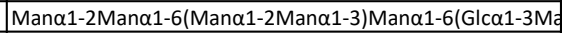 & -6 & 2 & -35 \\
\hline 40 & EY-15 & Man $\alpha 1-6($ Man $\alpha 1-3)$ Man $\alpha 1-6($ GIcNAc $\beta 1-4)(G I c N A c \beta 1-2 M a r$ & -13 & 10 & -76 \\
\hline 41 & EY-16 & GIcNAc $\beta 1-2 M a n \alpha 1-6(G I c N A c \beta 1-2 M a n \alpha 1-3)$ Man $\beta 1-4 G I c N A$ & -10 & 4 & -41 \\
\hline 42 & EY-26 & GlcNAc $\beta 1-2 M a n \alpha 1-6(G I c N A c \beta 1-4)(G I c N A c \beta 1-2 M a n \alpha 1-3) M$ & 63599 & 940 & 1 \\
\hline 43 & EY-32 & GIcNAc $\beta 1-6($ GIcNAc $\beta 1-4)(G I c N A c \beta 1-2) M a n \alpha 1-6(G I c N A c \beta 1-$ & 20 & 7 & 37 \\
\hline 44 & 2'-FL & Fuco1-2Galß1-4Glc & 60616 & 3398 & 6 \\
\hline 45 & 6'-SL & 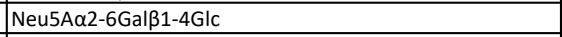 & -4 & 3 & -77 \\
\hline 46 & EY-20 & 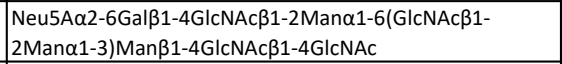 & 96 & 222 & 231 \\
\hline 47 & Man8 & $\begin{array}{l}\text { Man } \alpha 1-2 \text { Man } \alpha 1-6 \text { (Man } \alpha 1-2 \text { Man } \alpha 1-3) \text { Man } \alpha 1-6(\text { Man } \alpha 1- \\
\text { 2Man } 1-3) \text { Man } \beta 1-4 G I c N A c \beta 1-4 G I c N A c\end{array}$ & -9 & 3 & -29 \\
\hline 48 & FBS-1 & $\begin{array}{l}\text { Neu5A } \alpha 2-3 \text { Gal } \beta 1-4 G I c N A c \beta 1-2 M a n \alpha 1-6(\text { Neu5A } \alpha 2-3 G a l \beta 1- \\
\text { 4GIcNAc } \beta 1-4((\text { Neu5A } \alpha 2-3 G a l \beta 1-4 G I c N A c \beta 1-2) M a n \alpha 1- \\
\text { 3)Man } \beta 1-4 G I c N A c \beta 1-4 G l c N A c\end{array}$ & 7 & 31 & 422 \\
\hline 49 & Water & & 8 & 4 & 50 \\
\hline 50 & Water & & -3 & 3 & -92 \\
\hline 51 & Water & & 1 & 3 & 283 \\
\hline 52 & Biotin-BSA & & 19844 & 4322 & 22 \\
\hline
\end{tabular}


Table S4. Printed microarray raw data. Column A to C list the ID, name and structure of printed glycans; Column D is the average relative fluorescent units (RFU); Column E is the standard deviation of 4 printed spots; Column F is the ratio between STDEV and average RFU.

\begin{tabular}{|c|c|c|c|c|c|}
\hline \multicolumn{6}{|c|}{ AAL: $0.1 \mathrm{ug} / \mathrm{mL}$} \\
\hline Glycan ID & Glycan name & Structure & Average & STDEV & $\% \mathrm{CV}$ \\
\hline 1 & Lacto-N-tetraose (LNT) & Galß1-3GIcNacß1-3Galß1-4GIc & 119 & 6 & 5 \\
\hline 2 & Lacto-N-neotetraose (LNnT / neo-LNT) & Galß1-4GIcNAcß1-3Galß1-4GIc & 71 & 28 & 39 \\
\hline 3 & Blood group $\mathrm{H}$ antigen pentaose type 2 / Lacto-N-neofucopentaose I (LnNFP I) & Fuc $\alpha 1-2$ Gal $\beta 1-4$ GIcNAc $\beta 1-3 G a l \beta 1-4 G I c$ & 56799 & 2255 & 4 \\
\hline 4 & Blood group A Lewisb antigen pentaose type 1 & GalNAc $\alpha 1-3$ (Fuc $\alpha 1-2$ )Galß1-3(Fuc $\alpha 1-4)$ GIcNAc & 61107 & 1162 & 2 \\
\hline 5 & Blood group A LewisY antigen pentaose type 2 & GaINAc $\alpha 1-3$ (Fuca1-2)Galß1-4(Fuc $\alpha 1-3)$ GIcNAc & 58809 & 1405 & 2 \\
\hline 6 & Blood group $\mathrm{A}$ antigen pentaose type 1 & GalNAc $\alpha 1-3($ Fuc $\alpha 1-2)$ Gal $\beta 1-3 G I c N A c \beta 1-3 G a l$ & 15 & 11 & 77 \\
\hline 7 & Blood group $\mathrm{A}$ antigen pentaose type 2 & GalNAca1-3(Fuc $\alpha 1-2)$ Galß1-4GIcNAc $\beta 1-3 G a l$ & -35 & 51 & -146 \\
\hline 8 & Blood group $A$ antigen hexaose type 1 & GalNAc $\alpha 1-3$ (Fuc $\alpha 1-2)$ Gal $\beta 1-3 G I c N A c \beta 1-3 G a l \beta 1-4 G I c$ & 3 & 5 & 182 \\
\hline 9 & Blood group $\mathrm{A}$ antigen hexaose type 2 & GalNAc $\alpha 1-3$ (Fuc $\alpha 1-2)$ Gal $\beta 1-4 G I c N A c \beta 1-3 G a l \beta 1-4 G I c$ & 8 & 48 & 580 \\
\hline 10 & Blood group B Lewisb antigen pentaose type 1 & Gal $\alpha 1-3$ (Fuc $\alpha 1-2)$ Galß1-3(Fuc $\alpha 1-4) G I c N A c$ & 61148 & 2786 & 5 \\
\hline 11 & Blood group B LewisY antigen pentaose type 2 & Gal $\alpha 1-3$ (Fuc $\alpha 1-2$ )Galß1-4(Fuc $\alpha 1-3)$ GIcNAc & 59742 & 1743 & 3 \\
\hline 12 & Blood group B antigen pentaose type 1 & 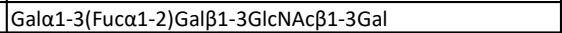 & 6 & 9 & 166 \\
\hline 13 & Blood group B antigen pentaose type 2 & 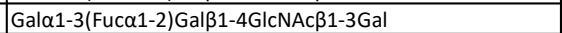 & 0 & 1 & $\# \mathrm{DIV} / 0$ ! \\
\hline 14 & Blood group B antigen hexaose type 1 & Gal $\alpha 1-3$ (Fuc $\alpha 1-2)$ Galß1-3GIcNAc $\beta 1-3 G a|\beta 1-4 G| c$ & 2 & 8 & 439 \\
\hline 15 & Blood group B antigen hexaose type 2 & Gal $\alpha 1-3$ (Fuc $\alpha 1-2)$ Gal $\beta 1-4 G|c N A c \beta 1-3 G a l \beta 1-4 G| c$ & 14 & 5 & 38 \\
\hline 16 & LewisX (LeX) tetraose & Galß1-4( Fuc $\alpha 1-3)$ GIcNAc $\beta 1-3 G a l$ & 61053 & 731 & 1 \\
\hline 17 & LewisY (LeY) pentaose & Fuc $\alpha 1-2$ Gal $\beta 1-4($ Fuc $\alpha 1-3)$ GlcNAc $\beta 1-3 G a l$ & 56963 & 750 & 1 \\
\hline 18 & Lewisa (Lea) tetraose & Gal $\beta 1-3$ (Fuc $\alpha 1-4)$ GIcNAc $\beta 1-3 G a l$ & 60731 & 1265 & 2 \\
\hline 19 & Lewisb (Leb) pentaose & Fuc $\alpha 1-2$ Gal $\beta 1-3$ (Fuc $\alpha 1-4)$ GlcNAc $\beta 1-3 G a l$ & 58975 & 770 & 1 \\
\hline 20 & Galili antigen pentaose & Gal $\alpha 1-3$ Gal $\beta 1-4 G I c N A c \beta 1-3 G a l \beta 1-4 G I c$ & 4 & 6 & 157 \\
\hline 21 & Isoglobopentaose / iGb5 & 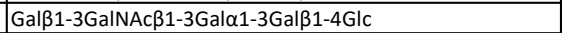 & 1 & 4 & 424 \\
\hline 22 & Galili antigen heptaose & Gal $\alpha 1-3($ Gal$\beta 1-4 G I c N A c \beta 1-3) 2 G a|\beta 1-4 G| c$ & 10 & 7 & 65 \\
\hline 23 & Globotriaose (Gb3) / Pk antigen & 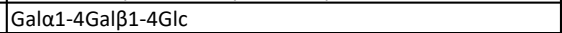 & 2 & 5 & 238 \\
\hline 24 & Globotetraose (Gb4) / $P$ antigen & 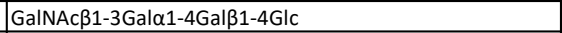 & 8 & 4 & 49 \\
\hline 25 & Globo-H hexaose / Stage Specific Embryonic Antigen 3b (SSEA-3b) & Fuc $\alpha 1-2$ Gal $\beta 1-3 G a l N A c \beta 1-3 G a l \alpha 1-4 G a l \beta 1-4 G \mid c$ & 10860 & 359 & 3 \\
\hline 26 & Globo-A heptaose & 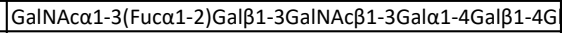 & 4 & 1 & 30 \\
\hline 27 & Globo-B heptaose & 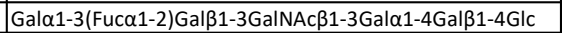 & -2 & 2 & -118 \\
\hline 28 & Blood group $\mathrm{H}$ antigen tetraose type 4 / Globo $\mathrm{H}$ tetraose & Fuc $\alpha 1-2$ Gal $\beta 1-3 G a I N A c \beta 1-3 G a l$ & 55193 & 4654 & 8 \\
\hline 29 & Blood group $\mathrm{A}$ antigen pentaose type 4 & 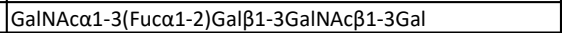 & 8 & 25 & 310 \\
\hline 30 & Blood group B antigen pentasaccharide type 4 & Gal $\alpha 1-3$ (Fuc $\alpha 1-2)$ Galß1-3GalNAc $\beta 1-3 G a l$ & -4 & 2 & -54 \\
\hline 31 & Forssman antigen pentaose & GaINAc $\alpha 1-3$ GalNAc $\beta 1-3 G a l \alpha 1-4 G a \mid \beta 1-4 G I c$ & -5 & 18 & -379 \\
\hline 32 & Isoforssman antigen pentaose & GalNAc $\alpha 1-3$ GalNAc $\beta 1-3 G a l \alpha 1-3 G a l \beta 1-4 G \mid c$ & -1 & 16 & -3292 \\
\hline 33 & Globotriaose analogue type 2/ P1 antigen & Gal 1 1-4Galß1-4GIcNAc & 116 & 20 & 17 \\
\hline 34 & Isoglobo- $\mathrm{H}$ analogue type 1 & Fuc $\alpha 1-2$ Gal $\beta 1-3 G I c N A c \beta 1-3 G a l \alpha 1-3 G a l \beta 1-4 G l c$ & 59166 & 2799 & 5 \\
\hline 35 & OL-05 & GIcNAc $\beta 1-6($ GIcNAc $\beta 1-4)(G \mid c N A c \beta 1-2)$ Man $\alpha 1-6($ GIcNAc $\beta 1-$ & 7 & 1 & 9 \\
\hline 36 & OL-09 & Man $\alpha 1-6($ Man $\alpha 1-3)$ Man $\alpha 1-6($ GIcNAc $\beta 1-4)(G I c N A c \beta 1-4(G I C$ & -8 & 6 & -83 \\
\hline 37 & $\mathrm{OL}-13$ & 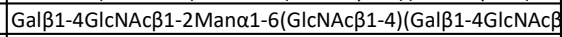 & -4 & 4 & -102 \\
\hline 38 & $\mathrm{OL}-17$ & GlcNAc $\beta 1-4(G I c N A c \beta 1-2)$ Man $\alpha 1-6(G I c N A c \beta 1-4)(G I c N A c \beta 1-$ & -13 & 7 & -59 \\
\hline 39 & EY-10 & 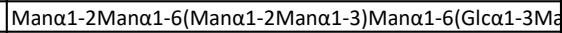 & 10 & 11 & 111 \\
\hline 40 & EY-15 & Man $\alpha 1-6($ Man $\alpha 1-3)$ Man $\alpha 1-6($ GIcNAc $\beta 1-4)(G I c N A c \beta 1-2 M a r$ & -30 & 10 & -33 \\
\hline 41 & EY-16 & GIcNAc $\beta 1-2 M a n \alpha 1-6(G I c N A c \beta 1-2 M a n \alpha 1-3)$ Man $\beta 1-4 G I c N A$ & 5 & 12 & 239 \\
\hline 42 & EY-26 & GlcNAc $\beta 1-2 M a n \alpha 1-6(G l c N A c \beta 1-4)(G l c N A c \beta 1-2 M a n \alpha 1-3) M$ & 58553 & 1184 & 2 \\
\hline 43 & EY-32 & GlcNAc $\beta 1-6($ GIcNAc $\beta 1-4)(G \mid c N A c \beta 1-2)$ Man $\alpha 1-6($ GlcNAc $\beta 1-$ & 203 & 26 & 13 \\
\hline 44 & $2^{\prime}-\mathrm{FL}$ & Fuc $\alpha 1-2$ Gal $\beta 1-4 G l c$ & 59968 & 1010 & 2 \\
\hline 45 & 6'-SL & 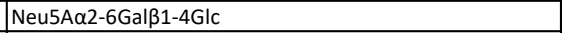 & -4 & 4 & -106 \\
\hline 46 & EY-20 & 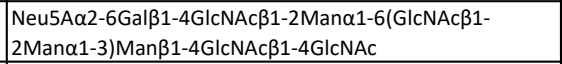 & -13 & 2 & -14 \\
\hline 47 & Man8 & 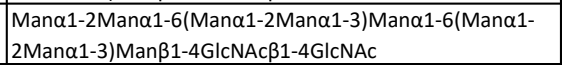 & 1 & 8 & 1069 \\
\hline 48 & FBS-1 & $\begin{array}{l}\text { Neu5A } \alpha 2-3 \text { Gal } \beta 1-4 G I c N A c \beta 1-2 M a n \alpha 1-6(\text { Neu5A } \alpha 2-3 G a l \beta 1- \\
\text { 4GIcNAc } \beta 1-4((N e u 5 A \alpha 2-3 G a l \beta 1-4 G I c N A c \beta 1-2) \text { Man } \alpha 1- \\
\text { 3)Man } \beta 1-4 G I c N A c \beta 1-4 G I c N A c\end{array}$ & -8 & 33 & -447 \\
\hline 49 & Water & & -6 & 4 & -74 \\
\hline 50 & Water & & -6 & 2 & -41 \\
\hline 51 & Water & & -11 & 13 & -117 \\
\hline 52 & Biotin-BSA & & 16640 & 4583 & 28 \\
\hline
\end{tabular}


Table S4. Printed microarray raw data. Column A to C list the ID, name and structure of printed glycans; Column D is the average relative fluorescent units (RFU); Column E is the standard deviation of 4 printed spots; Column $\mathrm{F}$ is the ratio between STDEV and average RFU.

\begin{tabular}{|c|c|c|c|c|c|}
\hline \multicolumn{6}{|c|}{ AAL: $1 \mathrm{ug} / \mathrm{mL}$} \\
\hline Glycan ID & Glycan name & Structure & Average & STDEV & $\% \mathrm{CV}$ \\
\hline 1 & Lacto-N-tetraose (LNT) & Galß1-3GIcNacß1-3Galß1-4GIc & 237 & 53 & 22 \\
\hline 2 & Lacto-N-neotetraose (LNnT / neo-LNT) & Galß1-4GIcNAcß1-3Galß1-4GIc & 106 & 47 & 44 \\
\hline 3 & Blood group $\mathrm{H}$ antigen pentaose type 2 / Lacto-N-neofucopentaose I (LnNFP I) & Fuc $\alpha 1-2$ Gal $\beta 1-4$ GIcNAc $\beta 1-3 G a l \beta 1-4 G I c$ & 60659 & 841 & 1 \\
\hline 4 & Blood group A Lewisb antigen pentaose type 1 & GalNAc $\alpha 1-3$ (Fuc $\alpha 1-2$ )Galß1-3(Fuc $\alpha 1-4)$ GIcNAc & 61994 & 1513 & 2 \\
\hline 5 & Blood group A LewisY antigen pentaose type 2 & GalNAc $\alpha 1-3$ (Fuc $\alpha 1-2$ )Gal $\beta 1-4($ Fuc $\alpha 1-3)$ GIcNAc & 60387 & 1896 & 3 \\
\hline 6 & Blood group $A$ antigen pentaose type 1 & 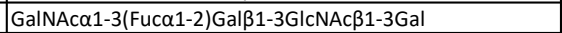 & 28 & 10 & 34 \\
\hline 7 & Blood group $A$ antigen pentaose type 2 & GalNAca1-3(Fuc $\alpha 1-2)$ Galß1-4GIcNAc $\beta 1-3 G a l$ & 19 & 11 & 57 \\
\hline 8 & Blood group $\mathrm{A}$ antigen hexaose type 1 & GalNAc $\alpha 1-3$ (Fuc $\alpha 1-2)$ Gal $\beta 1-3 G I c N A c \beta 1-3 G a l \beta 1-4 G I c$ & 0 & 5 & \#DIV/0! \\
\hline 9 & Blood group $\mathrm{A}$ antigen hexaose type 2 & GalNAc $\alpha 1-3$ (Fuc $\alpha 1-2)$ Gal $\beta 1-4 G I c N A c \beta 1-3 G a l \beta 1-4 G I c$ & 87 & 15 & 17 \\
\hline 10 & Blood group B Lewisb antigen pentaose type 1 & Gal $\alpha 1-3$ (Fuc $\alpha 1-2)$ Galß1-3(Fuc $\alpha 1-4) G I c N A c$ & 60312 & 2377 & 4 \\
\hline 11 & Blood group B LewisY antigen pentaose type 2 & Gal $\alpha 1-3$ (Fuc $\alpha 1-2$ )Galß1-4(Fuc $\alpha 1-3)$ GIcNAc & 61153 & 1340 & 2 \\
\hline 12 & Blood group B antigen pentaose type 1 & 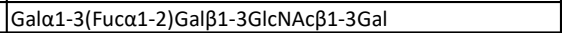 & 1 & 6 & 499 \\
\hline 13 & Blood group $B$ antigen pentaose type 2 & 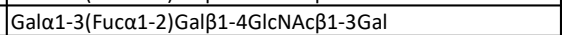 & 155 & 13 & 9 \\
\hline 14 & Blood group B antigen hexaose type 1 & Gal $\alpha 1-3$ (Fuc $\alpha 1-2)$ Galß1-3GIcNAc $\beta 1-3 G a|\beta 1-4 G| c$ & 11 & 4 & 34 \\
\hline 15 & Blood group B antigen hexaose type 2 & Gal $\alpha 1-3$ (Fuc $\alpha 1-2)$ Gal $\beta 1-4 G|c N A c \beta 1-3 G a l \beta 1-4 G| c$ & 2891 & 247 & 9 \\
\hline 16 & LewisX (LeX) tetraose & Gal $\beta 1-4$ ( Fuc $\alpha 1-3)$ GIcNAc $\beta 1-3 G a l$ & 63663 & 1690 & 3 \\
\hline 17 & LewisY (LeY) pentaose & 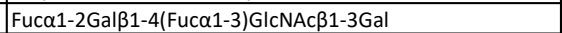 & 55241 & 2169 & 4 \\
\hline 18 & Lewisa (Lea) tetraose & Gal $\beta 1-3$ (Fuc $\alpha 1-4)$ GIcNAc $\beta 1-3 G a l$ & 60116 & 1219 & 2 \\
\hline 19 & Lewisb (Leb) pentaose & Fuc $\alpha 1-2$ Gal $\beta 1-3($ Fuc $\alpha 1-4)$ GlcNAc $\beta 1-3 \mathrm{Gal}$ & 57432 & 3205 & 6 \\
\hline 20 & Galili antigen pentaose & Gal $\alpha 1-3$ Gal $\beta 1-4 G I c N A c \beta 1-3 G a l \beta 1-4 G I c$ & 2 & 2 & 91 \\
\hline 21 & Isoglobopentaose / iGb5 & 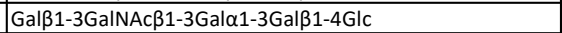 & 1 & 6 & 516 \\
\hline 22 & Galili antigen heptaose & Gal $\alpha 1-3($ Gal$\beta 1-4 G I c N A c \beta 1-3) 2 G a|\beta 1-4 G| c$ & 44 & 36 & 83 \\
\hline 23 & Globotriaose (Gb3) / Pk antigen & Gala1-4Galß1-4GIc & 10 & 7 & 66 \\
\hline 24 & Globotetraose (Gb4) / P antigen & GalNAc $\beta 1-3 G$ Gal $\alpha 1-4$ Gal $\beta 1-4 G \mid c$ & 16 & 5 & 35 \\
\hline 25 & Globo-H hexaose / Stage Specific Embryonic Antigen 3b (SSEA-3b) & Fuc $\alpha 1-2$ Gal $\beta 1-3 G a l N A c \beta 1-3 G a l \alpha 1-4 G a l \beta 1-4 G \mid c$ & 57273 & 3761 & 7 \\
\hline 26 & Globo-A heptaose & 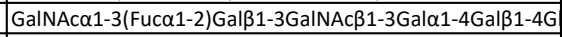 & 12 & 6 & 52 \\
\hline 27 & Globo-B heptaose & 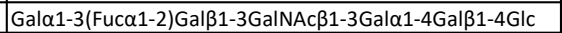 & 7 & 10 & 148 \\
\hline 28 & Blood group $\mathrm{H}$ antigen tetraose type 4 / Globo $\mathrm{H}$ tetraose & Fuc $\alpha 1-2$ Gal $\beta 1-3 G$ GINAc $\beta 1-3 G a l$ & 62737 & 1271 & 2 \\
\hline 29 & Blood group $A$ antigen pentaose type 4 & 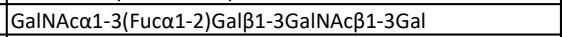 & 3 & 1 & 46 \\
\hline 30 & Blood group B antigen pentasaccharide type 4 & Gal $\alpha 1-3$ (Fuc $\alpha 1-2)$ Galß1-3GalNAc $\beta 1-3 G a l$ & 4 & 5 & 129 \\
\hline 31 & Forssman antigen pentaose & GaINAc $\alpha 1-3$ GaINAc $\beta 1-3 G a l \alpha 1-4 G a \mid \beta 1-4 G I c$ & 2 & 7 & 441 \\
\hline 32 & Isoforssman antigen pentaose & GalNAc $\alpha 1-3$ GalNAc $\beta 1-3 G a l \alpha 1-3 G a l \beta 1-4 G \mid c$ & 7 & 4 & 52 \\
\hline 33 & Globotriaose analogue type 2/ P1 antigen & Gal 1 1-4Galß1-4GIcNAc & 343 & 19 & 6 \\
\hline 34 & Isoglobo- $\mathrm{H}$ analogue type 1 & Fuc $\alpha 1-2$ Gal $\beta 1-3 G I c N A c \beta 1-3 G a l \alpha 1-3 G a l \beta 1-4 G l c$ & 61226 & 2361 & 4 \\
\hline 35 & OL-05 & GIcNAc $\beta 1-6(G I c N A c \beta 1-4)(G \mid c N A c \beta 1-2) M a n \alpha 1-6(G I c N A c \beta 1-$ & 24 & 21 & 89 \\
\hline 36 & OL-09 & Man $\alpha 1-6($ Man $\alpha 1-3)$ Man $\alpha 1-6($ GIcNAc $\beta 1-4)(G I c N A c \beta 1-4(G I C$ & 33 & 53 & 162 \\
\hline 37 & OL-13 & 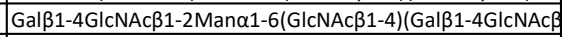 & 5 & 5 & 121 \\
\hline 38 & OL-17 & GIcNAc $\beta 1-4($ GIcNAc $\beta 1-2)$ Man $\alpha 1-6($ GIcNAc $\beta 1-4)(G I c N A c \beta 1-$ & -3 & 5 & -158 \\
\hline 39 & EY-10 & 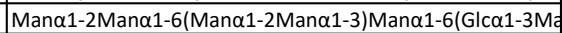 & -1 & 6 & -473 \\
\hline 40 & EY-15 & Man $\alpha 1-6($ Man $\alpha 1-3)$ Man $\alpha 1-6($ GIcNAc $\beta 1-4)(G I c N A c \beta 1-2 M a r$ & -15 & 2 & -10 \\
\hline 41 & EY-16 & GIcNAc $\beta 1-2 M a n \alpha 1-6(G I c N A c \beta 1-2 M a n \alpha 1-3)$ Man $\beta 1-4 G I c N A$ & 3 & 11 & 360 \\
\hline 42 & EY-26 & GIcNAc $\beta 1-2 M a n \alpha 1-6(G \mid c N A c \beta 1-4)(G I c N A c \beta 1-2 M a n \alpha 1-3) M$ & 62353 & 1513 & 2 \\
\hline 43 & EY-32 & GIcNAc $\beta 1-6($ GIcNAc $\beta 1-4)($ GIcNAc $\beta 1-2)$ Man $\alpha 1-6($ GIcNAc $\beta 1-$ & 459 & 42 & 9 \\
\hline 44 & 2'-FL & Fuc $\alpha 1-2$ Gal $\beta 1-4 G l c$ & 55212 & 1119 & 2 \\
\hline 45 & 6'-SL & 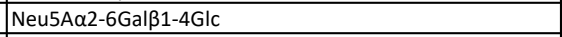 & 5 & 6 & 113 \\
\hline 46 & EY-20 & 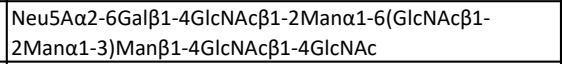 & -7 & 7 & -101 \\
\hline 47 & Man8 & 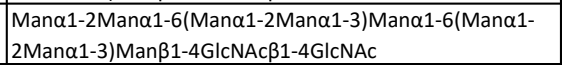 & 2 & 6 & 338 \\
\hline 48 & FBS-1 & $\begin{array}{l}\text { Neu5A } \alpha 2-3 \text { Gal } \beta 1-4 G I c N A c \beta 1-2 M a n \alpha 1-6(\text { Neu5A } \alpha 2-3 G a l \beta 1- \\
\text { 4GIcNAc } \beta 1-4((\text { Neu5A } \alpha 2-3 G a l \beta 1-4 G I c N A c \beta 1-2) M a n \alpha 1- \\
\text { 3)Man } \beta 1-4 G I c N A c \beta 1-4 G I c N A c\end{array}$ & -13 & 2 & -17 \\
\hline 49 & Water & & 12 & 7 & 61 \\
\hline 50 & Water & & 2 & 3 & 207 \\
\hline 51 & Water & & 5 & 4 & 85 \\
\hline 52 & Biotin-BSA & & 14231 & 4468 & 31 \\
\hline
\end{tabular}


Table S4. Printed microarray raw data. Column A to C list the ID, name and structure of printed glycans; Column D is the average relative fluorescent units (RFU); Column E is the standard deviation of 4 printed spots; Column $\mathrm{F}$ is the ratio between STDEV and average RFU.

\begin{tabular}{|c|c|c|c|c|c|}
\hline \multicolumn{6}{|c|}{ RCA-I: $0.1 \mathrm{ug} / \mathrm{mL}$} \\
\hline Glycan ID & Glycan name & Structure & Average & STDEV & $\% \mathrm{CV}$ \\
\hline 1 & Lacto-N-tetraose (LNT) & 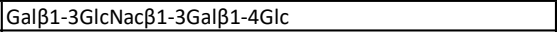 & 101 & 33 & 32 \\
\hline 2 & Lacto-N-neotetraose (LNnT / neo-LNT) & Galß1-4GIcNAcß1-3Galß1-4GIc & 316 & 79 & 25 \\
\hline 3 & Blood group $\mathrm{H}$ antigen pentaose type 2 / Lacto-N-neofucopentaose I (LnNFP I) & Fuc $\alpha 1-2$ Gal $\beta 1-4 G I c N A c \beta 1-3 G a l \beta 1-4 G l c$ & 30 & 30 & 100 \\
\hline 4 & Blood group A Lewisb antigen pentaose type 1 & GalNAc $\alpha 1-3$ (Fuc $\alpha 1-2)$ Galß1-3(Fuc $\alpha 1-4)$ GIcNAc & 17 & 9 & 52 \\
\hline 5 & Blood group A LewisY antigen pentaose type 2 & GalNAc $\alpha 1-3$ (Fuc $\alpha 1-2)$ Galß1-4(Fuc $\alpha 1-3)$ GIcNAc & 7 & 13 & 180 \\
\hline 6 & Blood group A antigen pentaose type 1 & GalNAc $\alpha 1-3$ (Fuc $\alpha 1-2)$ Gal $\beta 1-3 G I c N A c \beta 1-3 G a l$ & 5 & 4 & 83 \\
\hline 7 & Blood group $\mathrm{A}$ antigen pentaose type 2 & GalNAc $\alpha 1-3$ (Fuc $\alpha 1-2)$ Gal $\beta 1-4$ GIcNAc $\beta 1-3 G a l$ & 16 & 16 & 102 \\
\hline 8 & Blood group A antigen hexaose type 1 & GalNAc $\alpha 1-3($ Fuc $\alpha 1-2)$ Gal $\beta 1-3 G I c N A c \beta 1-3 G a l \beta 1-4 G \mid c$ & 7 & 8 & 118 \\
\hline 9 & Blood group A antigen hexaose type 2 & GalNAc $\alpha 1-3($ Fuc $\alpha 1-2)$ Gal $\beta 1-4$ GlcNAc $\beta 1-3 G a l \beta 1-4 G l c$ & 12 & 25 & 218 \\
\hline 10 & Blood group B Lewisb antigen pentaose type 1 & Gal $\alpha 1-3$ (Fuc $\alpha 1-2$ )Galß1-3(Fuc $\alpha 1-4)$ GIcNAc & 0 & 5 & -2151 \\
\hline 11 & Blood group B LewisY antigen pentaose type 2 & Gal $\alpha 1-3$ (Fuc $\alpha 1-2)$ Galß1-4(Fuc $\alpha 1-3)$ GIcNAc & -14 & 40 & -293 \\
\hline 12 & Blood group B antigen pentaose type 1 & 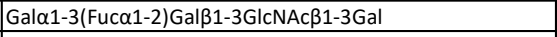 & 4 & 2 & 59 \\
\hline 13 & Blood group B antigen pentaose type 2 & 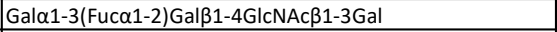 & 8 & 10 & 129 \\
\hline 14 & Blood group B antigen hexaose type 1 & Gal $\alpha 1-3$ (Fuc $\alpha 1-2)$ Gal $\beta 1-3$ GIcNAc $\beta 1-3 G a l \beta 1-4 G l c$ & -4 & 5 & -122 \\
\hline 15 & Blood group B antigen hexaose type 2 & Gal $\alpha 1-3$ (Fuc $\alpha 1-2)$ Gal $\beta 1-4$ GIcNAc $\beta 1-3 G a l \beta 1-4 G l c$ & -65 & 124 & -191 \\
\hline 16 & LewisX (LeX) tetraose & Gal $\beta 1-4$ ( Fuc $\alpha 1-3$ )GlcNAc $\beta 1-3 G a l$ & -69 & 136 & -198 \\
\hline 17 & LewisY (LeY) pentaose & Fuc $\alpha 1-2$ Gal $\beta 1-4$ (Fuc $\alpha 1-3)$ GlcNAc $\beta 1-3 G$ al & 2 & 7 & 408 \\
\hline 18 & Lewisa (Lea) tetraose & Gal $\beta 1-3$ (Fuc $\alpha 1-4)$ GIcNAc $\beta 1-3 G a l$ & 7 & 3 & 37 \\
\hline 19 & Lewisb (Leb) pentaose & Fuc $\alpha 1-2$ Gal $\beta 1-3$ (Fuc $\alpha 1-4$ )GlcNAc $\beta 1-3 \mathrm{Gal}$ & -16 & 34 & -218 \\
\hline 20 & Galili antigen pentaose & 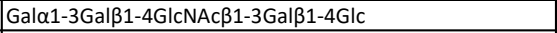 & 9 & 12 & 129 \\
\hline 21 & Isoglobopentaose / iGb5 & Galß1-3GalNAcß1-3Gal $\alpha 1-3 G a l \beta 1-4 G l c$ & 5 & 9 & 199 \\
\hline 22 & Galili antigen heptaose & 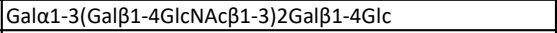 & 2 & 2 & 76 \\
\hline 23 & Globotriaose (Gb3) / Pk antigen & Gala1-4Galß1-4Glc & 7 & 3 & 47 \\
\hline 24 & Globotetraose (Gb4) / $P$ antigen & GalNAc $\beta 1-3$ Gal $\alpha 1-4$ Gal $\beta 1-4 G I c$ & -4 & 8 & -198 \\
\hline 25 & Globo-H hexaose / Stage Specific Embryonic Antigen 3b (SSEA-3b) & 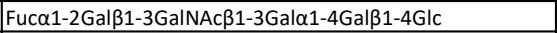 & 3 & 3 & 88 \\
\hline 26 & Globo-A heptaose & GalNAc $\alpha 1-3$ (Fuc $\alpha 1-2)$ Gal $\beta 1-3$ GalNAc $\beta 1-3 G a l \alpha 1-4 G a l \beta 1-4 G$ & 2 & 5 & 235 \\
\hline 27 & Globo-B heptaose & 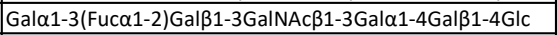 & -3 & 8 & -304 \\
\hline 28 & Blood group $\mathrm{H}$ antigen tetraose type 4 / Globo $\mathrm{H}$ tetraose & Fuc $\alpha 1-2$ Gal $\beta 1-3 G a l N A c \beta 1-3 G a l$ & -13 & 5 & -39 \\
\hline 29 & Blood group $\mathrm{A}$ antigen pentaose type 4 & GalNAc $\alpha 1-3$ (Fuc $\alpha 1-2)$ Gal $\beta 1-3$ GalNAc $\beta 1-3 G a l$ & -1 & 5 & -455 \\
\hline 30 & Blood group B antigen pentasaccharide type 4 & Gal $\alpha 1-3($ Fuc $\alpha 1-2)$ Gal $\beta 1-3 G a l N A c \beta 1-3 G a l$ & 6 & 9 & 137 \\
\hline 31 & Forssman antigen pentaose & GalNAc $\alpha 1-3$ GalNAc $\beta 1-3 G a l \alpha 1-4 G a l \beta 1-4 G I c$ & -4 & 14 & -368 \\
\hline 32 & Isoforssman antigen pentaose & GaINAc $\alpha 1-3 G a l N A c \beta 1-3 G a l \alpha 1-3 G a l \beta 1-4 G \mid c$ & -6 & 5 & -80 \\
\hline 33 & Globotriaose analogue type 2/ P1 antigen & Gal $\alpha 1-4$ Galß1-4GIcNAc & -1 & 5 & -931 \\
\hline 34 & Isoglobo- $\mathrm{H}$ analogue type 1 & Fuc $\alpha 1-2$ Gal $\beta 1-3$ GlcNAc $\beta 1-3 G a l \alpha 1-3 G a|\beta 1-4 G| c$ & 7 & 5 & 78 \\
\hline 35 & OL-05 & GIcNAc $\beta 1-6($ GlcNAc $\beta 1-4)($ GlcNAc $\beta 1-2)$ Man $\alpha 1-6(G I c N A c \beta 1-$ & 6 & 17 & 287 \\
\hline 36 & OL-09 & Man $\alpha 1-6($ Man $\alpha 1-3)$ Man $\alpha 1-6($ GlcNAc $\beta 1-4)($ GlcNAc $\beta 1-4(G I C$ & c-7 & 18 & -263 \\
\hline 37 & OL-13 & Gal $\beta 1-4 G \mid c N A c \beta 1-2 M a n \alpha 1-6(G \mid c N A c \beta 1-4)(G a l \beta 1-4 G \mid c N A c \beta$ & 154 & 70 & 46 \\
\hline 38 & OL-17 & GlcNAc $\beta 1-4(G \mid c N A c \beta 1-2)$ Man $\alpha 1-6($ GlcNAc $\beta 1-4)(G I c N A c \beta 1-$ & -1 & 11 & -1462 \\
\hline 39 & EY-10 & 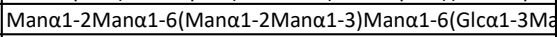 & -3 & 6 & -215 \\
\hline 40 & EY-15 & Man $\alpha 1-6($ Man $\alpha 1-3)$ Man $\alpha 1-6($ GlcNAc $\beta 1-4)($ GlcNAc $\beta 1-2 M a r$ & 18 & 45 & 250 \\
\hline 41 & EY-16 & GlcNAc $\beta 1-2$ Man $\alpha 1-6$ (GlcNAc $\beta 1-2 M a n \alpha 1-3)$ Man $\beta 1-4 G I c N A$ & 2 & 12 & 667 \\
\hline 42 & EY-26 & GlcNAc $\beta 1-2 M a n \alpha 1-6(G I c N A c \beta 1-4)(G I c N A c \beta 1-2 M a n \alpha 1-3) M$ & 23 & 8 & 34 \\
\hline 43 & EY-32 & GIcNAc $\beta 1-6(G \mid c N A c \beta 1-4)($ GIcNAc $\beta 1-2)$ Man $\alpha 1-6(G \mid c N A c \beta 1-$ & 1 & 3 & 529 \\
\hline 44 & 2'-FL & Fuc $\alpha 1-2$ Galß1-4Glc & -5 & 13 & -283 \\
\hline 45 & 6'-SL & Neu5A $\alpha 2-6$ Galß1-4Glc & -1 & 11 & -915 \\
\hline 46 & EY-20 & 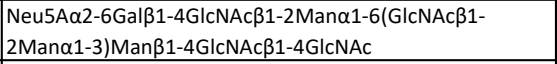 & 70 & 11 & 16 \\
\hline 47 & Man8 & 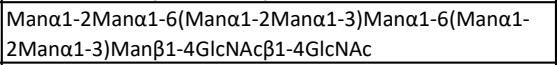 & -2 & 2 & -82 \\
\hline 48 & FBS-1 & $\begin{array}{l}\text { Neu5A } \alpha 2-3 \text { Gal } \beta 1-4 G I c N A c \beta 1-2 M a n \alpha 1-6(N e u 5 A \alpha 2-3 G a l \beta 1- \\
\text { 4GIcNAc } \beta 1-4((\text { Neu5A } \alpha 2-3 G a l \beta 1-4 G I c N A c \beta 1-2) \text { Man } \alpha 1- \\
\text { 3)Man } \beta 1-4 G I c N A c \beta 1-4 G I c N A c\end{array}$ & 27159 & 5974 & 22 \\
\hline 49 & Water & & -3 & 8 & -309 \\
\hline 50 & Water & & -6 & 10 & -190 \\
\hline 51 & Water & & -4 & 2 & -40 \\
\hline 52 & Biotin-BSA & & 12658 & 3609 & 29 \\
\hline
\end{tabular}


Table S4. Printed microarray raw data. Column A to C list the ID, name and structure of printed glycans; Column D is the average relative fluorescent units (RFU); Column E is the standard deviation of 4 printed spots; Column F is the ratio between STDEV and average RFU.

\begin{tabular}{|c|c|c|c|c|c|}
\hline \multicolumn{6}{|c|}{ RCA-I: 1ug/mL } \\
\hline Glycan ID & Glycan name & Structure & Average & STDEV & $\% \mathrm{CV}$ \\
\hline 1 & Lacto-N-tetraose (LNT) & Galß1-3GIcNac $\beta 1-3 G a l \beta 1-4 G \mid c$ & 257 & 53 & 21 \\
\hline 2 & Lacto-N-neotetraose (LNnT / neo-LNT) & 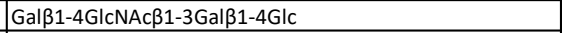 & 12842 & 659 & 5 \\
\hline 3 & Blood group $\mathrm{H}$ antigen pentaose type 2 / Lacto-N-neofucopentaose I (LnNFP I) & Fuc $\alpha 1-2$ Gal $\beta 1-4$ GlcNAc $\beta 1-3 G a \mid \beta 1-4 G l c$ & 36 & 13 & 36 \\
\hline 4 & Blood group A Lewisb antigen pentaose type 1 & GalNAc $\alpha 1-3$ (Fuc $\alpha 1-2$ )Galß1-3(Fuc $\alpha 1-4)$ GIcNAc & 32 & 16 & 51 \\
\hline 5 & Blood group A LewisY antigen pentaose type 2 & GalNAc $\alpha 1-3$ (Fuc $\alpha 1-2$ )Galß1-4(Fuc $\alpha 1-3)$ GIcNAc & 20 & 9 & 44 \\
\hline 6 & Blood group A antigen pentaose type 1 & 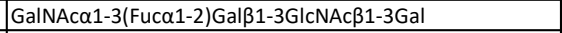 & 21 & 5 & 23 \\
\hline 7 & Blood group $\mathrm{A}$ antigen pentaose type 2 & GalNAc $\alpha 1-3($ Fuc $\alpha 1-2)$ Gal $\beta 1-4 G I c N A c \beta 1-3 G a l$ & 22 & 11 & 51 \\
\hline 8 & Blood group A antigen hexaose type 1 & GalNAc $\alpha 1-3$ (Fuc $\alpha 1-2)$ Gal $\beta 1-3$ GIcNAc $\beta 1-3 G a l \beta 1-4 G l c$ & 13 & 5 & 36 \\
\hline 9 & Blood group $A$ antigen hexaose type 2 & GalNAc $\alpha 1-3$ (Fuc $\alpha 1-2)$ Gal $\beta 1-4 G I c N A c \beta 1-3 G a l \beta 1-4 G I c$ & 5 & 13 & 270 \\
\hline 10 & Blood group B Lewisb antigen pentaose type 1 & Gal $\alpha 1-3$ (Fuc $\alpha 1-2$ )Gal $\beta 1-3$ (Fuc $\alpha 1-4)$ GlcNAc & 7 & 7 & 105 \\
\hline 11 & Blood group B LewisY antigen pentaose type 2 & Gala1-3(Fuc $\alpha 1-2)$ Gal $\beta 1-4($ Fuc $\alpha 1-3)$ GIcNAc & 3 & 4 & 158 \\
\hline 12 & Blood group B antigen pentaose type 1 & Gal $\alpha 1-3$ (Fuc $\alpha 1-2$ )Gal $\beta 1-3 G \mid c N A c \beta 1-3 G a l$ & 9 & 4 & 52 \\
\hline 13 & Blood group B antigen pentaose type 2 & Gal $\alpha 1-3$ (Fuc $\alpha 1-2$ )Gal $\beta 1-4 G \mid c N A c \beta 1-3 G a l$ & 13 & 7 & 53 \\
\hline 14 & Blood group B antigen hexaose type 1 & Gal $\alpha 1-3$ (Fuc $\alpha 1-2)$ Gal $\beta 1-3$ GlcNAc $\beta 1-3 G a l \beta 1-4 G l c$ & 5 & 3 & 61 \\
\hline 15 & Blood group B antigen hexaose type 2 & Gal $\alpha 1-3$ (Fuc $\alpha 1-2)$ Gal $\beta 1-4 G|c N A c \beta 1-3 G a l \beta 1-4 G| c$ & 6 & 4 & 74 \\
\hline 16 & LewisX (LeX) tetraose & Gal $\beta 1-4$ ( Fuc $\alpha 1-3)$ GIcNAc $\beta 1-3 G a l$ & 8 & 2 & 19 \\
\hline 17 & LewisY (LeY) pentaose & Fuc $\alpha 1-2$ Gal $\beta 1-4($ Fuc $\alpha 1-3)$ GlcNAc $\beta 1-3 G a l$ & 4 & 9 & 212 \\
\hline 18 & Lewisa (Lea) tetraose & Galß1-3(Fuc $\alpha 1-4)$ GIcNAc $\beta 1-3 G a l$ & 7 & 7 & 96 \\
\hline 19 & Lewisb (Leb) pentaose & Fuc $\alpha 1-2$ Gal $\beta 1-3$ (Fuc $\alpha 1-4)$ GlcNAc $\beta 1-3 G a l$ & 11 & 4 & 38 \\
\hline 20 & Galili antigen pentaose & Gal $\alpha 1-3 G a l \beta 1-4 G|c N A c \beta 1-3 G a l \beta 1-4 G| c$ & 24 & 6 & 26 \\
\hline 21 & Isoglobopentaose / iGb5 & Gal $\beta 1-3 G a l N A c \beta 1-3 G a l \alpha 1-3 G a l \beta 1-4 G l c$ & 35 & 19 & 54 \\
\hline 22 & Galili antigen heptaose & Gal $\alpha 1-3($ Gal$\beta 1-4 G \mid c N A c \beta 1-3) 2 G a l \beta 1-4 G \mid c$ & 19 & 6 & 32 \\
\hline 23 & Globotriaose (Gb3) / Pk antigen & Gala1-4Galß1-4GIc & 14 & 5 & 34 \\
\hline 24 & Globotetraose (Gb4) / $P$ antigen & GalNAc $\beta 1-3 G a l \alpha 1-4 G a|\beta 1-4 G| c$ & 24 & 28 & 117 \\
\hline 25 & Globo-H hexaose / Stage Specific Embryonic Antigen 3b (SSEA-3b) & Fuc $\alpha 1-2$ Gal $\beta 1-3 G a l N A c \beta 1-3 G a l \alpha 1-4 G a l \beta 1-4 G l c$ & 5 & 19 & 419 \\
\hline 26 & Globo-A heptaose & 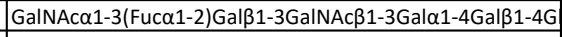 & 7 & 4 & 60 \\
\hline 27 & Globo-B heptaose & Gal $\alpha 1-3($ Fuc $\alpha 1-2)$ Galß1-3GalNAc $\beta 1-3 G a l \alpha 1-4 G a l \beta 1-4 G l c$ & 46 & 30 & 67 \\
\hline 28 & Blood group $\mathrm{H}$ antigen tetraose type 4 / Globo $\mathrm{H}$ tetraose & Fuc $\alpha 1-2$ Gal $\beta 1-3 G a I N A c \beta 1-3 G a l$ & 3 & 5 & 200 \\
\hline 29 & Blood group A antigen pentaose type 4 & GalNAc $\alpha 1-3($ Fuc $\alpha 1-2)$ Gal $\beta 1-3 G a l N A c \beta 1-3 G a l$ & 10 & 16 & 164 \\
\hline 30 & Blood group B antigen pentasaccharide type 4 & Gal $\alpha 1-3$ (Fuc $\alpha 1-2$ )Galß1-3GalNAc $\beta 1-3 G a l$ & 11 & 13 & 115 \\
\hline 31 & Forssman antigen pentaose & GalNAc $\alpha 1-3 G a l N A c \beta 1-3 G a l \alpha 1-4 G a l \beta 1-4 G \mid c$ & 7 & 14 & 204 \\
\hline 32 & Isoforssman antigen pentaose & GaINAc $\alpha 1-3$ GalNAc $\beta 1-3 G a l \alpha 1-3 G a l \beta 1-4 G I c$ & 4 & 7 & 174 \\
\hline 33 & Globotriaose analogue type 2/ $\mathrm{P} 1$ antigen & 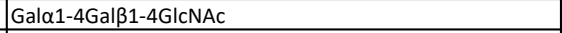 & 9 & 15 & 167 \\
\hline 34 & Isoglobo- $\mathrm{H}$ analogue type 1 & Fuc $\alpha 1-2$ Gal $\beta 1-3 G I c N A c \beta 1-3 G a l \alpha 1-3 G a l \beta 1-4 G l c$ & 12 & 5 & 37 \\
\hline 35 & OL-05 & GlcNAc $\beta 1-6($ GIcNAc $\beta 1-4)(G \mid c N A c \beta 1-2)$ Man $\alpha 1-6($ GlcNAc $\beta 1-$ & 24 & 15 & 62 \\
\hline 36 & OL-09 & Man $\alpha 1-6($ Man $\alpha 1-3)$ Man $\alpha 1-6($ GlcNAc $\beta 1-4)(G l c N A c \beta 1-4(G l c$ & 12 & 15 & 130 \\
\hline 37 & $\mathrm{OL}-13$ & Gal $\beta 1-4$ GIcNAc $\beta 1-2 M a n \alpha 1-6(G \mid c N A c \beta 1-4)(G a l \beta 1-4 G \mid c N A c \beta$ & 10227 & 1202 & 12 \\
\hline 38 & OL-17 & GlcNAc $\beta 1-4(G I c N A c \beta 1-2) M a n \alpha 1-6(G l c N A c \beta 1-4)(G l c N A c \beta 1-$ & 3 & 7 & 233 \\
\hline 39 & EY-10 & 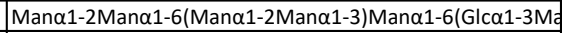 & 11 & 15 & 140 \\
\hline 40 & EY-15 & Man $\alpha 1-6($ Man $\alpha 1-3)$ Man $\alpha 1-6($ GlcNAc $\beta 1-4)(G l c N A c \beta 1-2 M a r$ & 4 & 4 & 101 \\
\hline 41 & EY-16 & GlcNAc $\beta 1-2 M a n \alpha 1-6(G l c N A c \beta 1-2 M a n \alpha 1-3)$ Man $\beta 1-4 G I c N A$ & 0 & 6 & \#DIV/0! \\
\hline 42 & EY-26 & GIcNAc $\beta 1-2$ Man $\alpha 1-6($ GlcNAc $\beta 1-4)(G I c N A c \beta 1-2 M a n \alpha 1-3) M$ & 253 & 25 & 10 \\
\hline 43 & EY-32 & GlcNAc $\beta 1-6(G I c N A c \beta 1-4)(G l c N A c \beta 1-2) M a n \alpha 1-6(G l c N A c \beta 1-$ & 15 & 11 & 75 \\
\hline 44 & 2'-FL & Fuc $\alpha 1-2$ Galß1-4Glc & -1 & 4 & -825 \\
\hline 45 & 6'-SL & 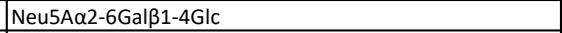 & 8 & 219 & 2739 \\
\hline 46 & EY-20 & $\begin{array}{l}\text { Neu5A } \alpha 2-6 \text { Gal } \beta 1-4 G \mid c N A c \beta 1-2 M a n \alpha 1-6 \text { (GIcNAc } \beta 1- \\
\text { 2Man } \alpha 1-3) \text { Man } \beta 1-4 G l c N A c \beta 1-4 G I c N A c\end{array}$ & 8255 & 833 & 10 \\
\hline 47 & Man8 & 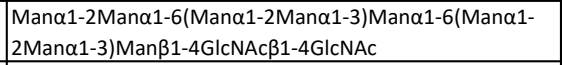 & 0 & 9 & 3583 \\
\hline 48 & FBS-1 & 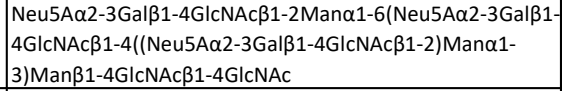 & 59897 & 3611 & 6 \\
\hline 49 & Water & & -196 & 264 & -135 \\
\hline 50 & Water & & \begin{tabular}{|l|}
-24 \\
\end{tabular} & 15 & -64 \\
\hline 51 & Water & & 10 & 22 & 224 \\
\hline 52 & Biotin-BSA & & 9543 & 1156 & 12 \\
\hline
\end{tabular}


Table S4. Printed microarray raw data. Column A to C list the ID, name and structure of printed glycans; Column D is the average relative fluorescent units (RFU); Column E is the standard deviation of 4 printed spots; Column $\mathrm{F}$ is the ratio between STDEV and average RFU.

\begin{tabular}{|c|c|c|c|c|c|}
\hline \multicolumn{6}{|c|}{ BPL: $0.1 \mathrm{ug} / \mathrm{mL}$} \\
\hline Glycan ID & Glycan name & Structure & Average & STDEV & $\% \mathrm{CV}$ \\
\hline 1 & Lacto-N-tetraose (LNT) & 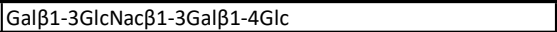 & 4995 & 348 & 7 \\
\hline 2 & Lacto-N-neotetraose (LNnT / neo-LNT) & Galß1-4GIcNAcß1-3Galß1-4Glc & 1743 & 202 & 12 \\
\hline 3 & Blood group $\mathrm{H}$ antigen pentaose type 2 / Lacto-N-neofucopentaose I (LnNFP I) & Fuc $\alpha 1-2$ Gal $\beta 1-4 G I c N A c \beta 1-3 G a \mid \beta 1-4 G I c$ & 14 & 9 & 67 \\
\hline 4 & Blood group A Lewisb antigen pentaose type 1 & GalNAc $\alpha 1-3$ (Fuc $\alpha 1-2)$ Gal $\beta 1-3$ (Fuc $\alpha 1-4)$ GIcNAc & -207 & 269 & -130 \\
\hline 5 & Blood group A LewisY antigen pentaose type 2 & GalNAca1-3(Fuc $\alpha 1-2)$ Galß1-4(Fuc $\alpha 1-3)$ GIcNAc & 4 & 4 & 118 \\
\hline 6 & Blood group A antigen pentaose type 1 & GalNAc $\alpha 1-3$ (Fuc $\alpha 1-2)$ Gal $\beta 1-3$ GIcNAc $\beta 1-3 G a l$ & 36 & 58 & 160 \\
\hline 7 & Blood group $\mathrm{A}$ antigen pentaose type 2 & GalNAc $\alpha 1-3$ (Fuc $\alpha 1-2)$ Gal $\beta 1-4 G I c N A c \beta 1-3 G a l$ & 9 & 12 & 136 \\
\hline 8 & Blood group $\mathrm{A}$ antigen hexaose type 1 & 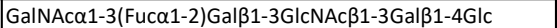 & 92 & 184 & 200 \\
\hline 9 & Blood group $\mathrm{A}$ antigen hexaose type 2 & GalNAc $\alpha 1-3$ (Fuc $\alpha 1-2)$ Gal $\beta 1-4 G I c N A c \beta 1-3 G a l \beta 1-4 G \mid c$ & 2 & 6 & 398 \\
\hline 10 & Blood group B Lewisb antigen pentaose type 1 & Gal $\alpha 1-3$ (Fuc $\alpha 1-2)$ Galß1-3(Fuc $\alpha 1-4)$ GIcNAc & 0 & 3 & -1361 \\
\hline 11 & Blood group B LewisY antigen pentaose type 2 & Gal $\alpha 1-3$ (Fuc $\alpha 1-2)$ Galß1-4(Fuc $\alpha 1-3)$ GIcNAc & 9 & 2 & 20 \\
\hline 12 & Blood group B antigen pentaose type 1 & 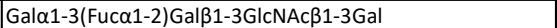 & -2 & 15 & -763 \\
\hline 13 & Blood group B antigen pentaose type 2 & Gal $\alpha 1-3$ (Fuc $\alpha 1-2)$ Gal $\beta 1-4 G \mid c N A c \beta 1-3 G a l$ & -10 & 8 & -81 \\
\hline 14 & Blood group B antigen hexaose type 1 & Gal $\alpha 1-3$ (Fuc $\alpha 1-2)$ Gal $\beta 1-3$ GIcNAc $\beta 1-3 G a l \beta 1-4 G \mid c$ & 16 & 31 & 193 \\
\hline 15 & Blood group $\mathrm{B}$ antigen hexaose type 2 & Gal $\alpha 1-3$ (Fuc $\alpha 1-2)$ Gal $\beta 1-4 G|c N A c \beta 1-3 G a l \beta 1-4 G| c$ & 2 & 5 & 248 \\
\hline 16 & LewisX (LeX) tetraose & Gal $\beta 1-4$ ( Fuc $\alpha 1-3)$ GlcNAc $\beta 1-3 G a l$ & 5615 & 678 & 12 \\
\hline 17 & LewisY (LeY) pentaose & Fuc $\alpha 1-2$ Gal $\beta 1-4($ Fuc $\alpha 1-3)$ GlcNAc $\beta 1-3 G a l$ & -5 & 10 & -203 \\
\hline 18 & Lewisa (Lea) tetraose & Galß1-3(Fuc $\alpha 1-4)$ GIcNAc $\beta 1-3 G a l$ & 80 & 10 & 13 \\
\hline 19 & Lewisb (Leb) pentaose & Fuc $\alpha 1-2$ Gal $\beta 1-3$ (Fuc $\alpha 1-4)$ GlcNAc $\beta 1-3 G a l$ & 1 & 7 & 873 \\
\hline 20 & Galili antigen pentaose & 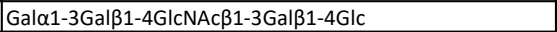 & 2432 & 152 & 6 \\
\hline 21 & Isoglobopentaose / iGb5 & Galß1-3GalNAc $\beta 1-3 G a l \alpha 1-3 G a l \beta 1-4 G l c$ & 15260 & 1948 & 13 \\
\hline 22 & Galili antigen heptaose & 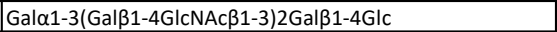 & 2652 & 135 & 5 \\
\hline 23 & Globotriaose (Gb3) / Pk antigen & 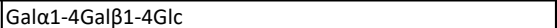 & -1 & 4 & -424 \\
\hline 24 & Globotetraose (Gb4) / P antigen & GalNAc $\beta 1-3 G a l \alpha 1-4 G a|\beta 1-4 G| c$ & 5997 & 311 & 5 \\
\hline 25 & Globo-H hexaose / Stage Specific Embryonic Antigen 3b (SSEA-3b) & 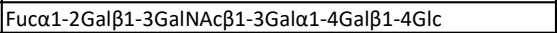 & -3 & 5 & -166 \\
\hline 26 & Globo-A heptaose & 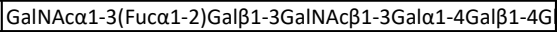 & -40 & 46 & -116 \\
\hline 27 & Globo-B heptaose & 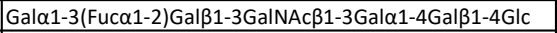 & -4 & 3 & -79 \\
\hline 28 & Blood group $\mathrm{H}$ antigen tetraose type 4 / Globo $\mathrm{H}$ tetraose & Fuc $\alpha 1-2$ Gal $\beta 1-3 G$ alNAc $\beta 1-3 G a l$ & 1 & 17 & 1726 \\
\hline 29 & Blood group $\mathrm{A}$ antigen pentaose type 4 & GalNAc $\alpha 1-3$ (Fuc $\alpha 1-2)$ Gal $\beta 1-3$ GalNAc $\beta 1-3 G a l$ & -28 & 54 & -197 \\
\hline 30 & Blood group B antigen pentasaccharide type 4 & Gal $\alpha 1-3$ (Fuc $\alpha 1-2)$ Galß1-3GalNAc $\beta 1-3 G a l$ & -16 & 11 & -68 \\
\hline 31 & Forssman antigen pentaose & GalNAc $\alpha 1-3$ GalNAc $\beta 1-3 G a l \alpha 1-4 G a l \beta 1-4 G I c$ & 11 & 4 & 39 \\
\hline 32 & Isoforssman antigen pentaose & GaINAc $\alpha 1-3$ GalNAc $\beta 1-3 G a l \alpha 1-3 G a l \beta 1-4 G \mid c$ & 25 & 9 & 38 \\
\hline 33 & Globotriaose analogue type 2/ P1 antigen & Gal $\alpha 1-4$ Galß1-4GIcNAc & -25 & 64 & -254 \\
\hline 34 & Isoglobo- $\mathrm{H}$ analogue type 1 & Fuc $\alpha 1-2$ Gal $\beta 1-3 G|c N A c \beta 1-3 G a| \alpha 1-3 G a|\beta 1-4 G| c$ & -12 & 12 & -97 \\
\hline 35 & OL-05 & GIcNAc $\beta 1-6($ GlcNAc $\beta 1-4)($ GlcNAc $\beta 1-2) M a n \alpha 1-6(G I c N A c \beta 1-$ & -5 & 15 & -333 \\
\hline 36 & OL-09 & Man $\alpha 1-6($ Man $\alpha 1-3)$ Man $\alpha 1-6($ GlcNAc $\beta 1-4)($ GlcNAc $\beta 1-4(G I C$ & 17 & 26 & 152 \\
\hline 37 & OL-13 & 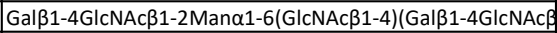 & 241 & 42 & 17 \\
\hline 38 & OL-17 & GlcNAc $\beta 1-4($ GlcNAc $\beta 1-2)$ Man $\alpha 1-6($ GlcNAc $\beta 1-4)(G \mid c N A c \beta 1-$ & -5 & 24 & -501 \\
\hline 39 & EY-10 & Man $\alpha 1-2 M a n \alpha 1-6(M a n \alpha 1-2 M a n \alpha 1-3) M a n \alpha 1-6(G l c \alpha 1-3 M$ & 52 & 63 & 121 \\
\hline 40 & EY-15 & Man $\alpha 1-6($ Man $\alpha 1-3)$ Man $\alpha 1-6($ GlcNAc $\beta 1-4)($ GIcNAc $\beta 1-2 M a r$ & 21 & 29 & 140 \\
\hline 41 & EY-16 & GlcNAc $\beta 1-2 M a n \alpha 1-6($ GlcNAc $\beta 1-2 M a n \alpha 1-3) M a n \beta 1-4 G I c N A$ & 7 & 11 & 156 \\
\hline 42 & EY-26 & GlcNAc $\beta 1-2 M a n \alpha 1-6($ GlcNAc $\beta 1-4)($ GlcNAc $\beta 1-2 M a n \alpha 1-3) M$ & -6 & 15 & -252 \\
\hline 43 & EY-32 & GlcNAc $\beta 1-6($ GlcNAc $\beta 1-4)($ GlcNAc $\beta 1-2) M a n \alpha 1-6(G \mid c N A c \beta 1-$ & -158 & 253 & -160 \\
\hline 44 & $2^{\prime}-\mathrm{FL}$ & Fuc $\alpha 1-2$ Galß1-4Glc & -133 & 228 & -172 \\
\hline 45 & 6'-SL & Neu5A $\alpha 2-6$ Gal $\beta 1-4 G I c$ & 4 & 8 & 184 \\
\hline 46 & EY-20 & $\begin{array}{l}\text { Neu5A } \alpha 2-6 \text { Gal } \beta 1-4 G \mid c N A c \beta 1-2 M a n \alpha 1-6(G \mid c N A c \beta 1- \\
2 \text { Man } \alpha 1-3) \text { Man } \beta 1-4 G \mid c N A c \beta 1-4 G I c N A c\end{array}$ & -11 & 9 & -84 \\
\hline 47 & Man8 & $\begin{array}{l}\text { Man } 11-2 \text { Man } \alpha 1-6(\text { Man } \alpha 1-2 \text { Man } \alpha 1-3) \text { Man } \alpha 1-6(\text { Man } \alpha 1- \\
\text { 2Man } 1 \text { 1-3)Man } \beta 1-4 G I c N A c \beta 1-4 G I c N A c\end{array}$ & -233 & 346 & -149 \\
\hline 48 & FBS-1 & 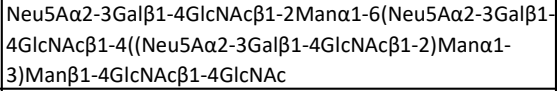 & -187 & 242 & -130 \\
\hline 49 & Water & & -2 & 10 & -462 \\
\hline 50 & Water & & 1 & 21 & 2838 \\
\hline 51 & Water & & -4 & 9 & -215 \\
\hline 52 & Biotin-BSA & & 8600 & 1805 & 21 \\
\hline
\end{tabular}


Table S4. Printed microarray raw data. Column A to C list the ID, name and structure of printed glycans; Column D is the average relative fluorescent units (RFU); Column E is the standard deviation of 4 printed spots; Column $\mathrm{F}$ is the ratio between STDEV and average RFU.

\begin{tabular}{|c|c|c|c|c|c|}
\hline \multicolumn{6}{|c|}{ BPL: 1ug/mL } \\
\hline Glycan ID & Glycan name & Structure & Average & STDEV & $\% \mathrm{CV}$ \\
\hline 1 & Lacto-N-tetraose (LNT) & 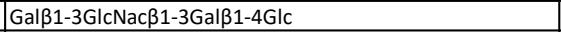 & 50757 & 965 & 2 \\
\hline 2 & Lacto-N-neotetraose (LNnT / neo-LNT) & 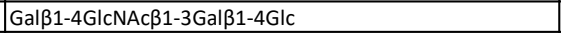 & 18845 & 1009 & 5 \\
\hline 3 & Blood group H antigen pentaose type 2 / Lacto-N-neofucopentaose I (LnNFP I) & Fuc $\alpha 1-2$ Gal $\beta 1-4$ GlcNAc $\beta 1-3 G a l \beta 1-4 G l c$ & 29 & 20 & 68 \\
\hline 4 & Blood group A Lewisb antigen pentaose type 1 & GalNAc $\alpha 1-3$ (Fuc $\alpha 1-2$ )Galß1-3(Fuc $\alpha 1-4)$ GlcNAc & 43 & 22 & 50 \\
\hline 5 & Blood group A LewisY antigen pentaose type 2 & GalNAc $\alpha 1-3$ (Fuc $\alpha 1-2$ )Galß1-4(Fuc $\alpha 1-3)$ GlcNAc & 53 & 120 & 227 \\
\hline 6 & Blood group $\mathrm{A}$ antigen pentaose type 1 & 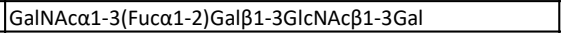 & 11 & 7 & 61 \\
\hline 7 & Blood group A antigen pentaose type 2 & 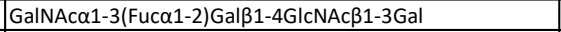 & 14 & 6 & 46 \\
\hline 8 & Blood group A antigen hexaose type 1 & GalNAc $\alpha 1-3$ (Fuc $\alpha 1-2$ )Gal $\beta 1-3$ GIcNAc $\beta 1-3 G a l \beta 1-4 G l c$ & 6 & 7 & 116 \\
\hline 9 & Blood group A antigen hexaose type 2 & GalNAc $\alpha 1-3$ (Fuc $\alpha 1-2$ )Gal $\beta 1-4$ GIcNAc $\beta 1-3 G a l \beta 1-4 G l c$ & 20 & 30 & 154 \\
\hline 10 & Blood group B Lewisb antigen pentaose type 1 & Gal $\alpha 1-3$ (Fuc $\alpha 1-2)$ Galß1-3(Fuc $\alpha 1-4)$ GIcNAc & 3 & 8 & 304 \\
\hline 11 & Blood group B LewisY antigen pentaose type 2 & Gal $\alpha 1-3$ (Fuc $\alpha 1-2)$ Galß 1 1-4(Fuc $\alpha 1-3)$ GlcNAc & 5 & 13 & 287 \\
\hline 12 & Blood group B antigen pentaose type 1 & Gal $\alpha 1-3$ (Fuc $\alpha 1-2$ )Gal $\beta 1-3 G \mid c N A c \beta 1-3 G a l$ & 3 & 10 & 415 \\
\hline 13 & Blood group B antigen pentaose type 2 & Gal $\alpha 1-3($ Fuc $\alpha 1-2)$ Gal $\beta 1-4 G \mid c N A c \beta 1-3 G a l$ & 8 & 2 & 27 \\
\hline 14 & Blood group B antigen hexaose type 1 & Gal $\alpha 1-3$ (Fuc $\alpha 1-2)$ Gal $\beta 1-3$ GlcNAc $\beta 1-3 G a|\beta 1-4 G| c$ & 17 & 31 & 180 \\
\hline 15 & Blood group B antigen hexaose type 2 & Gal $\alpha 1-3$ (Fuc $\alpha 1-2$ )Gal $\beta 1-4$ GlcNAc $\beta 1-3 G a l \beta 1-4 G \mid c$ & 7 & 1 & 12 \\
\hline 16 & LewisX (LeX) tetraose & Gal $\beta 1-4$ ( Fuc $\alpha 1-3)$ GlcNAc $\beta 1-3 G a l$ & 54418 & 492 & 1 \\
\hline 17 & LewisY (LeY) pentaose & Fuc $\alpha 1-2$ Gal $\beta 1-4$ (Fuc $\alpha 1-3)$ GlcNAc $\beta 1-3 G a l$ & -1 & 11 & -1134 \\
\hline 18 & Lewisa (Lea) tetraose & Gal $\beta 1-3$ (Fuc $\alpha 1-4)$ GlcNAc $\beta 1-3 G a l$ & 4852 & 516 & 11 \\
\hline 19 & Lewisb (Leb) pentaose & Fuc $\alpha 1-2$ Gal $\beta 1-3$ (Fuc $\alpha 1-4) G I c N A c \beta 1-3 G a l$ & 7 & 13 & 205 \\
\hline 20 & Galili antigen pentaose & Gal $\alpha 1-3$ Gal $\beta 1-4 G \mid c N A c \beta 1-3 G a l \beta 1-4 G l c$ & 29269 & 3383 & 12 \\
\hline 21 & Isoglobopentaose / iGb5 & Gal $\beta 1-3 G a I N A c \beta 1-3 G a l \alpha 1-3 G a l \beta 1-4 G \mid c$ & 60985 & 2926 & 5 \\
\hline 22 & Galili antigen heptaose & Gal $\alpha 1-3($ Gal $\beta 1-4 G \mid c N A c \beta 1-3) 2$ Galß1-4Glc & 35728 & 1277 & 4 \\
\hline 23 & Globotriaose (Gb3) / Pk antigen & 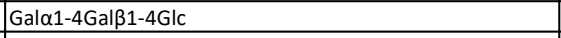 & 12 & 21 & 174 \\
\hline 24 & Globotetraose (Gb4) / $\mathrm{P}$ antigen & GalNAc $\beta 1-3$ Gal $\alpha 1-4$ Gal $\beta 1-4 G \mid c$ & 55802 & 4394 & 8 \\
\hline 25 & Globo-H hexaose / Stage Specific Embryonic Antigen 3b (SSEA-3b) & Fuc $\alpha 1-2$ Galß1-3GalNAc $\beta 1-3 G a l \alpha 1-4 G a l \beta 1-4 G \mid c$ & 3 & 16 & 567 \\
\hline 26 & Globo-A heptaose & 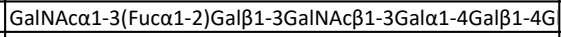 & 5 & 2 & 39 \\
\hline 27 & Globo-B heptaose & Gal $\alpha 1-3$ (Fuc $\alpha 1-2)$ Gal $\beta 1-3 G a l N A c \beta 1-3 G a l \alpha 1-4 G a|\beta 1-4 G| c$ & 20 & 52 & 266 \\
\hline 28 & Blood group $\mathrm{H}$ antigen tetraose type 4 / Globo $\mathrm{H}$ tetraose & Fuc $\alpha 1-2$ Gal $\beta 1-3 G$ alNAc $\beta 1-3 G a l$ & -104 & 133 & -128 \\
\hline 29 & Blood group $\mathrm{A}$ antigen pentaose type 4 & 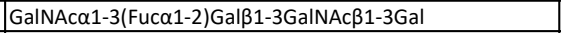 & 9 & 25 & 267 \\
\hline 30 & Blood group B antigen pentasaccharide type 4 & Gal $\alpha 1-3$ (Fuc $\alpha 1-2)$ Gal $\beta 1-3 G a l N A c \beta 1-3 G a l$ & 9 & 18 & 208 \\
\hline 31 & Forssman antigen pentaose & GalNAc $\alpha 1-3$ GalNAc $\beta 1-3 G a l \alpha 1-4 G a l \beta 1-4 G I c$ & 60 & 21 & 35 \\
\hline 32 & Isoforssman antigen pentaose & GalNAc $\alpha 1-3 G a I N A c \beta 1-3 G a l \alpha 1-3 G a l \beta 1-4 G \mid c$ & 55 & 78 & 142 \\
\hline 33 & Globotriaose analogue type 2/ P1 antigen & Gala1-4Galß1-4GIcNAc & 10 & 7 & 77 \\
\hline 34 & Isoglobo- $\mathrm{H}$ analogue type 1 & Fuc $\alpha 1-2$ Gal $\beta 1-3 G I c N A c \beta 1-3 G a l \alpha 1-3 G a l \beta 1-4 G l c$ & 8 & 5 & 73 \\
\hline 35 & $\mathrm{OL}-05$ & GlcNAc $\beta 1-6(G \mid c N A c \beta 1-4)(G I c N A c \beta 1-2)$ Man $\alpha 1-6(G I c N A c \beta 1-$ & 1 & 6 & 757 \\
\hline 36 & OL-09 & 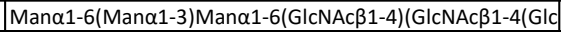 & 250 & 74 & 30 \\
\hline 37 & OL-13 & Gal $\beta 1-4 G I c N A c \beta 1-2 M a n \alpha 1-6(G \mid c N A c \beta 1-4)(G a l \beta 1-4 G \mid c N A c \beta$ & 6832 & 496 & 7 \\
\hline 38 & OL-17 & GlcNAc $\beta 1-4(G \mid c N A c \beta 1-2)$ Man $\alpha 1-6($ GlcNAc $\beta 1-4)(G l c N A c \beta 1-$ & 33 & 10 & 29 \\
\hline 39 & EY-10 & 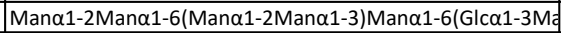 & 222 & 504 & 227 \\
\hline 40 & EY-15 & Man $\alpha 1-6$ (Man $\alpha 1-3)$ Man $\alpha 1-6(G l c N A c \beta 1-4)($ GlcNAc $\beta 1-2 M a r$ & -4 & 12 & -312 \\
\hline 41 & EY-16 & GlcNAcß1-2Man $\alpha 1-6(G l c N A c \beta 1-2 M a n \alpha 1-3)$ Man $\beta 1-4 G I c N A$ & 56 & 130 & 231 \\
\hline 42 & EY-26 & GlcNAc $\beta 1-2 M a n \alpha 1-6(G I c N A c \beta 1-4)(G I c N A c \beta 1-2 M a n \alpha 1-3) M$ & 27 & 22 & 80 \\
\hline 43 & EY-32 & GlcNAc $\beta 1-6($ GIcNAc $\beta 1-4)(G I c N A c \beta 1-2)$ Man $\alpha 1-6($ GlcNAc $\beta 1-$ & 41 & 145 & 353 \\
\hline 44 & 2'-FL & Fuc $\alpha 1-2$ Gal $\beta 1-4 G l c$ & -30 & 53 & -176 \\
\hline 45 & 6'-SL & Neu5A $\alpha 2-6$ Galß1-4Glc & 3 & 7 & 256 \\
\hline 46 & EY-20 & $\begin{array}{l}\text { Neu5A } \alpha 2-6 \text { Gal } \beta 1-4 G I c N A c \beta 1-2 M a n \alpha 1-6(G I c N A c \beta 1- \\
\text { 2Man } \alpha 1-3) \text { Man } \beta 1-4 G I c N A c \beta 1-4 G I c N A c\end{array}$ & -5 & 7 & -130 \\
\hline 47 & Man8 & 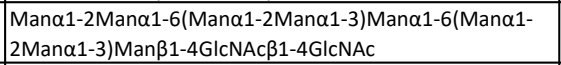 & 2 & 14 & 715 \\
\hline 48 & FBS-1 & $\begin{array}{l}\text { Neu5A } \alpha 2-3 \text { Gal } \beta 1-4 G I c N A c \beta 1-2 M a n \alpha 1-6(N e u 5 A \alpha 2-3 G a l \beta 1- \\
\text { 4GIcNAc } \beta 1-4((\text { Neu5A } \alpha 2-3 G a l \beta 1-4 G I c N A c \beta 1-2) \text { Man } \alpha 1- \\
\text { 3)Man } \beta 1-4 G I c N A c \beta 1-4 G I c N A c\end{array}$ & -42 & 55 & -130 \\
\hline 49 & Water & & 12 & 19 & 157 \\
\hline 50 & Water & & -2 & 5 & -200 \\
\hline 51 & Water & & -8 & 8 & -102 \\
\hline 52 & Biotin-BSA & & 6789 & 799 & 12 \\
\hline
\end{tabular}


Table S5. Printed microarray raw data. Column A to C list the ID, name and structure of printed glycans; Column D is the average relative fluorescent units (RFU); Column E is the standard deviation of 4 printed spots; Column F is the ratio between STDEV and average RFU.

\begin{tabular}{|c|c|c|c|c|c|}
\hline \multicolumn{6}{|c|}{ Anti-A antibody (1/100) } \\
\hline Glycan ID & Glycan name & Structure & Average & STDEV & $\% \mathrm{CV}$ \\
\hline 1 & Lacto-N-tetraose (LNT) & 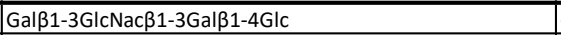 & -14 & 6 & -39 \\
\hline 2 & Lacto-N-neotetraose (LNnT / neo-LNT) & Galß1-4GIcNAcß1-3Galß1-4GIc & -15 & 9 & -56 \\
\hline 3 & Blood group $\mathrm{H}$ antigen pentaose type 2 / Lacto-N-neofucopentaose I (LnNFP I) & Fuc $\alpha 1-2$ Gal $\beta 1-4 G|c N A c \beta 1-3 G a l \beta 1-4 G| c$ & -19 & 10 & -51 \\
\hline 4 & Blood group A Lewisb antigen pentaose type 1 & GalNAc $\alpha 1-3$ (Fuc $\alpha 1-2)$ Galß1-3(Fuc $\alpha 1-4)$ GIcNAc & 62777 & 1327 & 2 \\
\hline 5 & Blood group A LewisY antigen pentaose type 2 & 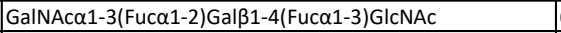 & 64793 & 280 & 0 \\
\hline 6 & Blood group $\mathrm{A}$ antigen pentaose type 1 & 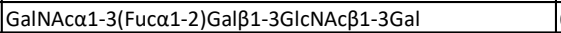 & 64144 & 457 & 1 \\
\hline 7 & Blood group $\mathrm{A}$ antigen pentaose type 2 & GalNAc $\alpha 1-3$ (Fuc $\alpha 1-2)$ Gal $\beta 1-4 G I c N A c \beta 1-3 G a l$ & 65159 & 271 & 0 \\
\hline 8 & Blood group $\mathrm{A}$ antigen hexaose type 1 & GalNAc $\alpha 1-3$ (Fuc $\alpha 1-2)$ Gal $\beta 1-3 G|c N A c \beta 1-3 G a l \beta 1-4 G| c$ & 64947 & 139 & 0 \\
\hline 9 & Blood group $A$ antigen hexaose type 2 & GalNAc $\alpha 1-3($ Fuc $\alpha 1-2)$ Gal $\beta 1-4 G I c N A c \beta 1-3 G a l \beta 1-4 G \mid c$ & 65296 & 163 & 0 \\
\hline 10 & Blood group B Lewisb antigen pentaose type 1 & Gal $\alpha 1-3$ (Fuc $\alpha 1-2)$ Galß1-3(Fuc $\alpha 1-4)$ GIcNAc & -10 & 11 & -109 \\
\hline 11 & Blood group B LewisY antigen pentaose type 2 & Gal $\alpha 1-3$ (Fuc $\alpha 1-2)$ Gal $\beta 1-4($ Fuc $\alpha 1-3)$ GlcNAc & -6 & 12 & -193 \\
\hline 12 & Blood group B antigen pentaose type 1 & 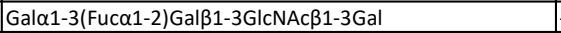 & -15 & 11 & -72 \\
\hline 13 & Blood group B antigen pentaose type 2 & 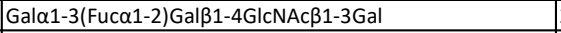 & 2 & 11 & 765 \\
\hline 14 & Blood group B antigen hexaose type 1 & Gal $\alpha 1-3$ (Fuc $\alpha 1-2)$ Gal $\beta 1-3$ GIcNAc $\beta 1-3 G a l \beta 1-4 G I c$ & 4 & 3 & 76 \\
\hline 15 & Blood group B antigen hexaose type 2 & Gal $\alpha 1-3$ (Fuc $\alpha 1-2)$ Gal $\beta 1-4$ GIcNAc $\beta 1-3 G a l \beta 1-4 G \mid c$ & 2 & 1 & 71 \\
\hline 16 & LewisX (LeX) tetraose & Gal $\beta 1-4$ ( Fuc $\alpha 1-3$ )GlcNAc $\beta 1-3 G a l$ & 5 & 5 & 113 \\
\hline 17 & LewisY (LeY) pentaose & 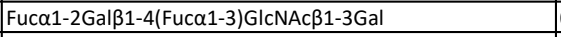 & 0 & 3 & -1194 \\
\hline 18 & Lewisa (Lea) tetraose & Gal $\beta 1-3$ (Fuc $\alpha 1-4$ )GIcNAc $\beta 1-3 G a l$ & 4 & 5 & 128 \\
\hline 19 & Lewisb (Leb) pentaose & Fuc $\alpha 1-2$ Gal $\beta 1-3$ (Fuc $\alpha 1-4)$ GIcNAc $\beta 1-3$ Gal & 2 & 3 & 164 \\
\hline 20 & Galili antigen pentaose & Gal $\alpha 1-3$ Gal $\beta 1-4 G I c N A c \beta 1-3 G a l \beta 1-4 G \mid c$ & 5 & 3 & 55 \\
\hline 21 & Isoglobopentaose / iGb5 & 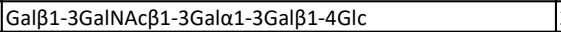 & 2 & 5 & 274 \\
\hline 22 & Galili antigen heptaose & 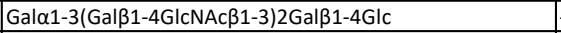 & -6 & 1 & -14 \\
\hline 23 & Globotriaose (Gb3) / Pk antigen & Gal $\alpha 1-4$ Galß1-4GIc & 5 & 5 & 91 \\
\hline 24 & Globotetraose (Gb4) / $P$ antigen & GalNAc $\beta 1-3 G a l \alpha 1-4 G a|\beta 1-4 G| c$ & 4 & 4 & 125 \\
\hline 25 & Globo-H hexaose / Stage Specific Embryonic Antigen 3b (SSEA-3b) & 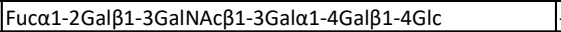 & -9 & 6 & -69 \\
\hline 26 & Globo-A heptaose & GalNAc $\alpha 1-3$ (Fuc $\alpha 1-2)$ Gal $\beta 1-3 G a l N A c \beta 1-3 G a l \alpha 1-4 G a l \beta 1-4 G$ & 64433 & 485 & 1 \\
\hline 27 & Globo-B heptaose & 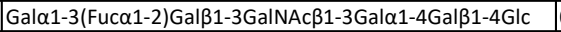 & 0 & 3 & \#DIV/0! \\
\hline 28 & Blood group $\mathrm{H}$ antigen tetraose type 4 / Globo $\mathrm{H}$ tetraose & Fuc $\alpha 1-2$ Gal $\beta 1-3$ GalNAc $\beta 1-3 G a l$ & 3 & 7 & 254 \\
\hline 29 & Blood group $\mathrm{A}$ antigen pentaose type 4 & GalNAc $\alpha 1-3$ (Fuc $\alpha 1-2)$ Gal $\beta 1-3 G a l N A c \beta 1-3 G a l$ & 64455 & 186 & 0 \\
\hline 30 & Blood group B antigen pentasaccharide type 4 & 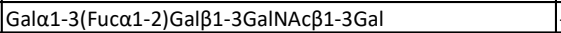 & -1 & 7 & -524 \\
\hline 31 & Forssman antigen pentaose & GalNAc $\alpha 1-3$ GalNAc $\beta 1-3$ Gal $\alpha 1-4$ Gal $\beta 1-4 G I c$ & 291 & 382 & 131 \\
\hline 32 & Isoforssman antigen pentaose & GalNAc $\alpha 1-3$ GalNAc $\beta 1-3 G a l \alpha 1-3 G a l \beta 1-4 G \mid c$ & 43 & 28 & 66 \\
\hline 33 & Globotriaose analogue type 2/ P1 antigen & Gal $\alpha 1-4$ Galß1-4GIcNAc & -3 & 5 & -213 \\
\hline 34 & Isoglobo-H analogue type 1 & Fuc $\alpha 1-2$ Gal $\beta 1-3 G I c N A c \beta 1-3 G a l \alpha 1-3 G a|\beta 1-4 G| c$ & 3 & 5 & 186 \\
\hline 35 & OL-05 & GlcNAc $\beta 1-6($ GlcNAc $\beta 1-4)(G I c N A c \beta 1-2)$ Man $\alpha 1-6(G l c N A c \beta 1-$ & 5 & 4 & 78 \\
\hline 36 & OL-09 & Man $\alpha 1-6($ Man $\alpha 1-3)$ Man $\alpha 1-6(G I c N A c \beta 1-4)(G l c N A c \beta 1-4$ (GIc & -1 & 3 & -230 \\
\hline 37 & OL-13 & Gal $\beta 1-4$ GIcNAc $\beta 1-2$ Man $\alpha 1-6($ GIcNAc $\beta 1-4)($ Gal $\beta 1-4 G I c N A c \beta$ & 6 & 6 & 100 \\
\hline 38 & OL-17 & GlcNAc $\beta 1-4($ GlcNAc $\beta 1-2) M a n \alpha 1-6(G I c N A c \beta 1-4)(G I c N A c \beta 1-$ & 2 & 4 & 296 \\
\hline 39 & EY-10 & 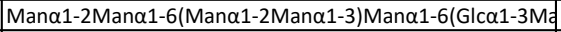 & 5 & 5 & 105 \\
\hline 40 & EY-15 & Man $\alpha 1-6($ Man $\alpha 1-3)$ Man $\alpha 1-6(G I c N A c \beta 1-4)(G I c N A c \beta 1-2 M a r$ & 2 & 4 & 172 \\
\hline 41 & EY-16 & GlcNAc $\beta 1-2$ Man $\alpha 1-6(G \mid c N A c \beta 1-2 M a n \alpha 1-3)$ Man $\beta 1-4 G I c N A$ & 6 & 7 & 118 \\
\hline 42 & EY-26 & GlcNAc $\beta 1-2 M a n \alpha 1-6(G I c N A c \beta 1-4)(G l c N A c \beta 1-2 M a n \alpha 1-3) M$ & 4 & 1 & 35 \\
\hline 43 & EY-32 & GlcNAc $\beta 1-6(G \mid c N A c \beta 1-4)(G I c N A c \beta 1-2) M a n \alpha 1-6(G l c N A c \beta 1-$ & 22 & 30 & 136 \\
\hline 44 & 2'-FL & Fuc $\alpha 1-2$ Galß1-4Glc & 7 & 3 & 34 \\
\hline 45 & 6'-SL & Neu5A $\alpha 2-6$ Gal $\beta 1-4 G I c$ & 5 & 1 & 16 \\
\hline 46 & EY-20 & 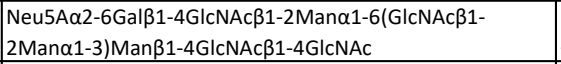 & -3 & 4 & -136 \\
\hline 47 & Man8 & $\begin{array}{l}\text { Man } \alpha 1-2 \text { Man } \alpha 1-6(\text { Man } \alpha 1-2 \text { Man } \alpha 1-3) \text { Man } \alpha 1-6(\text { Man } \alpha 1- \\
\text { 2Man } 1 \text { 1-3)Man } \beta 1-4 G I c N A c \beta 1-4 G I c N A c\end{array}$ & -1 & 4 & -491 \\
\hline 48 & FBS-1 & $\begin{array}{l}\text { Neu5A } \alpha 2-3 G a \mid \beta 1-4 G I c N A c \beta 1-2 M a n \alpha 1-6(\text { Neu5A } \alpha 2-3 G a l \beta 1- \\
\text { 4GIcNAc } \beta 1-4((\text { Neu5A } \alpha 2-3 G a l \beta 1-4 G I c N A c \beta 1-2) \text { Man } \alpha 1- \\
\text { 3)Man } \beta 1-4 G I c N A c \beta 1-4 G I c N A c\end{array}$ & -1 & 8 & -1519 \\
\hline 49 & Water & & 1 & 5 & 516 \\
\hline 50 & Water & & -3 & 5 & -210 \\
\hline 51 & Water & & -1 & 4 & -424 \\
\hline 52 & Biotin-BSA & & -7 & 23 & -353 \\
\hline
\end{tabular}


Table S5. Printed microarray raw data. Column A to C list the ID, name and structure of printed glycans; Column D is the average relative fluorescent units (RFU); Column E is the standard deviation of 4 printed spots; Column F is the ratio between STDEV and average RFU.

\begin{tabular}{|c|c|c|c|c|c|}
\hline \multicolumn{6}{|c|}{ Anti-B antibody (1/100) } \\
\hline Glycan ID & Glycan name & Structure & Average & STDEV & $\% \mathrm{CV}$ \\
\hline 1 & Lacto-N-tetraose (LNT) & 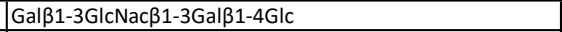 & 5 & 3 & 63 \\
\hline 2 & Lacto-N-neotetraose (LNnT / neo-LNT) & Gal $\beta 1-4 G I c N A c \beta 1-3 G a l \beta 1-4 G \mid c$ & 828 & 1648 & 199 \\
\hline 3 & Blood group $\mathrm{H}$ antigen pentaose type 2 / Lacto-N-neofucopentaose I (LnNFP I) & Fuc $\alpha 1-2$ Gal $\beta 1-4$ GIcNAc $\beta 1-3 G a l \beta 1-4 G l c$ & 4 & 4 & 100 \\
\hline 4 & Blood group A Lewisb antigen pentaose type 1 & GalNAc $\alpha 1-3$ (Fuc $\alpha 1-2$ )Galß1-3(Fuc $\alpha 1-4)$ GIcNAc & 6 & 3 & 46 \\
\hline 5 & Blood group A LewisY antigen pentaose type 2 & GaINAc $\alpha 1-3$ (Fuc $\alpha 1-2$ )Galß1-4(Fuc $\alpha 1-3)$ GIcNAc & 1 & 3 & 413 \\
\hline 6 & Blood group $A$ antigen pentaose type 1 & GalNAca1-3(Fuc $\alpha 1-2)$ Galß1-3GIcNAc $\beta 1-3 G a l$ & -2 & 4 & -172 \\
\hline 7 & Blood group $\mathrm{A}$ antigen pentaose type 2 & 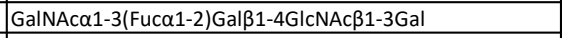 & -2 & 4 & -205 \\
\hline 8 & Blood group $A$ antigen hexaose type 1 & GalNAc $\alpha 1-3$ (Fuc $\alpha 1-2)$ Gal $\beta 1-3$ GIcNAc $\beta 1-3 G a l \beta 1-4 G l c$ & -3 & 6 & -207 \\
\hline 9 & Blood group $A$ antigen hexaose type 2 & GalNAc $\alpha 1-3$ (Fuc $\alpha 1-2)$ Gal $\beta 1-4 G I c N A c \beta 1-3 G a l \beta 1-4 G l c$ & -3 & 4 & -148 \\
\hline 10 & Blood group B Lewisb antigen pentaose type 1 & Gal $\alpha 1-3$ (Fuc $\alpha 1-2$ )Galß1-3(Fuc $\alpha 1-4)$ GlcNAc & 65355 & 108 & 0 \\
\hline 11 & Blood group B LewisY antigen pentaose type 2 & Gal $\alpha 1-3$ (Fuc $\alpha 1-2$ )Gal $\beta 1-4$ (Fuc $\alpha 1-3)$ GIcNAc & 65343 & 227 & 0 \\
\hline 12 & Blood group B antigen pentaose type 1 & Gal $\alpha 1-3$ (Fuc $\alpha 1-2$ )Galß1-3GlcNAc $\beta 1-3 G a l$ & 65471 & 25 & 0 \\
\hline 13 & Blood group B antigen pentaose type 2 & 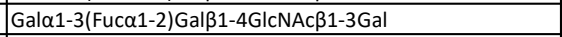 & 65172 & 83 & 0 \\
\hline 14 & Blood group B antigen hexaose type 1 & Gal $\alpha 1-3$ (Fuc $\alpha 1-2)$ Gal $\beta 1-3$ GlcNAc $\beta 1-3 G a l \beta 1-4 G l c$ & 64732 & 359 & 1 \\
\hline 15 & Blood group B antigen hexaose type 2 & Gal $\alpha 1-3($ Fuc $\alpha 1-2)$ Galß1-4GIcNAc $\beta 1-3 G a \mid \beta 1-4 G l c$ & 65194 & 460 & 1 \\
\hline 16 & LewisX (LeX) tetraose & Gal $\beta 1-4$ ( Fuc $\alpha 1-3$ )GIcNAc $\beta 1-3 G a l$ & -5 & 2 & -43 \\
\hline 17 & LewisY (LeY) pentaose & 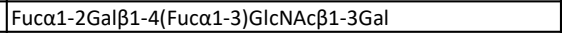 & 0 & 2 & -887 \\
\hline 18 & Lewisa (Lea) tetraose & Gal $\beta 1-3$ (Fuc $\alpha 1-4)$ GlcNAc $\beta 1-3 G a l$ & -4 & 2 & -40 \\
\hline 19 & Lewisb (Leb) pentaose & Fuc $\alpha 1-2$ Gal $\beta 1-3$ (Fuc $\alpha 1-4) G I c N A c \beta 1-3 G a l$ & -2 & 5 & -235 \\
\hline 20 & Galili antigen pentaose & Gal $\alpha 1-3$ Gal $\beta 1-4 G|c N A c \beta 1-3 G a l \beta 1-4 G| c$ & 4 & 2 & 49 \\
\hline 21 & Isoglobopentaose / iGb5 & Gal $\beta 1-3 G a l N A c \beta 1-3 G a l \alpha 1-3 G a l \beta 1-4 G l c$ & 3 & 3 & 133 \\
\hline 22 & Galili antigen heptaose & Gal $\alpha 1-3($ Gal $\beta 1-4 G \mid c N A c \beta 1-3) 2$ Gal $\beta 1-4 G \mid c$ & 4 & 2 & 35 \\
\hline 23 & Globotriaose (Gb3) / Pk antigen & Gala1-4Galß1-4GIc & 2 & 7 & 470 \\
\hline 24 & Globotetraose (Gb4) / P antigen & 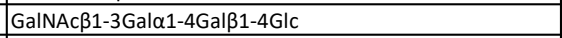 & 1 & 2 & 189 \\
\hline 25 & Globo-H hexaose / Stage Specific Embryonic Antigen 3b (SSEA-3b) & Fuc $\alpha 1-2$ Gal $\beta 1-3 G a l N A c \beta 1-3 G a l \alpha 1-4 G a l \beta 1-4 G l c$ & 4 & 6 & 166 \\
\hline 26 & Globo-A heptaose & 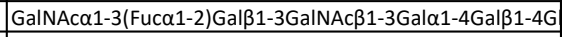 & 3 & 6 & 256 \\
\hline 27 & Globo-B heptaose & 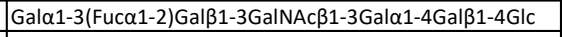 & 64985 & 677 & 1 \\
\hline 28 & Blood group $\mathrm{H}$ antigen tetraose type 4 / Globo $\mathrm{H}$ tetraose & 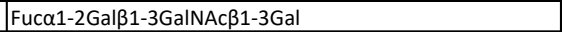 & 4 & 1 & 30 \\
\hline 29 & Blood group $\mathrm{A}$ antigen pentaose type 4 & GalNAca1-3(Fuc $\alpha 1-2)$ Galß1-3GalNAc $\beta 1-3 G a l$ & -2 & 6 & -314 \\
\hline 30 & Blood group B antigen pentasaccharide type 4 & Gal $\alpha 1-3$ (Fuc $\alpha 1-2$ )Gal $\beta 1-3 G a l N A c \beta 1-3 G a l$ & 64998 & 264 & 0 \\
\hline 31 & Forssman antigen pentaose & GalNAc $\alpha 1-3$ GalNAc $\beta 1-3 G a l \alpha 1-4 G a l \beta 1-4 G I c$ & 1 & 2 & 177 \\
\hline 32 & Isoforssman antigen pentaose & GaINAc $\alpha 1-3$ GalNAc $\beta 1-3 G a l \alpha 1-3 G a l \beta 1-4 G I c$ & 1 & 3 & 256 \\
\hline 33 & Globotriaose analogue type 2/ P1 antigen & 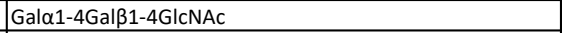 & -4 & 9 & -216 \\
\hline 34 & Isoglobo- $\mathrm{H}$ analogue type 1 & Fuc $\alpha 1-2$ Gal $\beta 1-3 G I c N A c \beta 1-3 G a l \alpha 1-3 G a l \beta 1-4 G \mid c$ & 0 & 3 & -1281 \\
\hline 35 & OL-05 & GIcNAc $\beta 1-6($ GIcNAc $\beta 1-4)(G \mid c N A c \beta 1-2)$ Man $\alpha 1-6($ GIcNAc $\beta 1-$ & 3 & 5 & 192 \\
\hline 36 & OL-09 & Man $\alpha 1-6($ Man $\alpha 1-3)$ Man $\alpha 1-6($ GlcNAc $\beta 1-4)(G I c N A c \beta 1-4(G I C$ & 5 & 4 & 81 \\
\hline 37 & $\mathrm{OL}-13$ & Gal $\beta 1-4$ GIcNAc $\beta 1-2 M a n \alpha 1-6(G \mid c N A c \beta 1-4)(G a l \beta 1-4 G I c N A c \beta$ & 3 & 6 & 204 \\
\hline 38 & OL-17 & GlcNAc $\beta 1-4($ GIcNAc $\beta 1-2)$ Man $\alpha 1-6(G I c N A c \beta 1-4)(G I c N A c \beta 1-$ & 8 & 9 & 117 \\
\hline 39 & EY-10 & 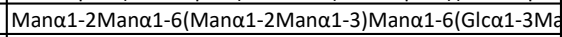 & 2 & 4 & 240 \\
\hline 40 & EY-15 & Man $\alpha 1-6$ (Man $\alpha 1-3)$ Man $\alpha 1-6($ GlcNAc $\beta 1-4)(G I c N A c \beta 1-2 M a r$ & 3 & 4 & 128 \\
\hline 41 & EY-16 & GlcNAc $\beta 1-2 M a n \alpha 1-6(G l c N A c \beta 1-2 M a n \alpha 1-3) M a n \beta 1-4 G I c N A$ & 4 & 2 & 46 \\
\hline 42 & EY-26 & GlcNAc $\beta 1-2$ Man $\alpha 1-6($ GlcNAc $\beta 1-4)(G l c N A c \beta 1-2 M a n \alpha 1-3) M$ & 1 & 2 & 476 \\
\hline 43 & EY-32 & GlcNAc $\beta 1-6($ GIcNAc $\beta 1-4)(G I c N A c \beta 1-2) M a n \alpha 1-6(G I c N A c \beta 1-$ & 0 & 5 & \#DIV/0! \\
\hline 44 & 2'-FL & Fuc $\alpha 1-2$ Galß1-4Glc & 4 & 3 & 88 \\
\hline 45 & 6 '-SL & Neu5A $\alpha 2-6$ Gal $\beta 1-4 G \mid c$ & 2 & 1 & 86 \\
\hline 46 & EY-20 & $\begin{array}{l}\text { Neu5A } \alpha 2-6 \text { Gal } \beta 1-4 G I c N A c \beta 1-2 M a n \alpha 1-6 \text { (GIcNAc } \beta 1- \\
2 \text { Man } \alpha 1-3) \text { Man } \beta 1-4 G I c N A c \beta 1-4 G I c N A c\end{array}$ & 0 & 3 & \#DIV/0! \\
\hline 47 & Man8 & 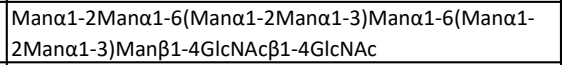 & 2 & 2 & 76 \\
\hline 48 & FBS-1 & $\begin{array}{l}\text { Neu5A } \alpha 2-3 \text { Gal } \beta 1-4 G I c N A c \beta 1-2 M a n \alpha 1-6(\text { Neu5A } \alpha 2-3 G a l \beta 1- \\
\text { 4GIcNAc } \beta 1-4((\text { Neu5A } \alpha 2-3 G a l \beta 1-4 G I c N A c \beta 1-2) M a n \alpha 1- \\
\text { 3)Man } \beta 1-4 G I c N A c \beta 1-4 G I c N A c\end{array}$ & 4 & 6 & 161 \\
\hline 49 & Water & & -1 & 4 & -872 \\
\hline 50 & Water & & 2 & 4 & 253 \\
\hline 51 & Water & & 3 & 3 & 105 \\
\hline 52 & Biotin-BSA & & 0 & 3 & -1322 \\
\hline
\end{tabular}


Table S5. Printed microarray raw data. Column A to C list the ID, name and structure of printed glycans; Column D is the average relative fluorescent units (RFU); Column E is the standard deviation of 4 printed spots; Column F is the ratio between STDEV and average RFU.

\begin{tabular}{|c|c|c|c|c|c|}
\hline \multicolumn{6}{|c|}{ Anti-Le ${ }^{a}$ antibody $(1 / 100)$} \\
\hline Glycan ID & Glycan name & Structure & Average & STDEV & $\% \mathrm{CV}$ \\
\hline 1 & Lacto-N-tetraose (LNT) & Galß1-3GIcNacß1-3Galß1-4GIc & -1 & 2 & -346 \\
\hline 2 & Lacto-N-neotetraose (LNnT / neo-LNT) & Galß1-4GIcNAcß1-3Galß1-4GIc & 3 & 5 & 166 \\
\hline 3 & Blood group $\mathrm{H}$ antigen pentaose type 2 / Lacto-N-neofucopentaose I (LnNFP I) & Fuc $\alpha 1-2$ Gal $\beta 1-4 G I c N A c \beta 1-3 G a l \beta 1-4 G I c$ & 4 & 2 & 68 \\
\hline 4 & Blood group A Lewisb antigen pentaose type 1 & GalNAc $\alpha 1-3$ (Fuc $\alpha 1-2)$ Gal $\beta 1-3($ Fuc $\alpha 1-4)$ GIcNAc & -3 & 3 & -124 \\
\hline 5 & Blood group A LewisY antigen pentaose type 2 & GalNAca1-3(Fuc $\alpha 1-2)$ Gal $\beta 1-4($ Fuc $\alpha 1-3)$ GIcNAc & 3 & 3 & 92 \\
\hline 6 & Blood group $\mathrm{A}$ antigen pentaose type 1 & GalNAc $\alpha 1-3$ (Fuc $\alpha 1-2$ )Gal $\beta 1-3$ GIcNAc $\beta 1-3 G a l$ & 2 & 3 & 147 \\
\hline 7 & Blood group $\mathrm{A}$ antigen pentaose type 2 & 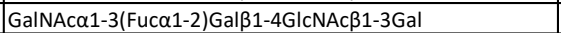 & 4 & 4 & 102 \\
\hline 8 & Blood group $A$ antigen hexaose type 1 & GalNAca1-3(Fuc $\alpha 1-2$ )Gal $\beta 1-3 G I c N A c \beta 1-3 G a l \beta 1-4 G I c$ & 4 & 3 & 76 \\
\hline 9 & Blood group $A$ antigen hexaose type 2 & GalNAc $\alpha 1-3$ (Fuc $\alpha 1-2$ )Gal $\beta 1-4$ GIcNAc $\beta 1-3$ Gal $\beta 1-4 G \mid c$ & 5 & 3 & 65 \\
\hline 10 & Blood group B Lewisb antigen pentaose type 1 & Gal $\alpha 1-3$ (Fuc $\alpha 1-2$ )Gal $\beta 1-3$ (Fuc $\alpha 1-4)$ GlcNAc & 3 & 2 & 62 \\
\hline 11 & Blood group B LewisY antigen pentaose type 2 & Gal $\alpha 1-3$ (Fuc $\alpha 1-2)$ Galß1-4(Fuc $\alpha 1-3)$ GIcNAc & 1 & 3 & 248 \\
\hline 12 & Blood group B antigen pentaose type 1 & Gal $\alpha 1-3$ (Fuc $\alpha 1-2$ )Gal $\beta 1-3 G \mid c N A c \beta 1-3 G a l$ & 29 & 64 & 218 \\
\hline 13 & Blood group B antigen pentaose type 2 & Gal $\alpha 1-3$ (Fuc $\alpha 1-2)$ Gal $\beta 1-4 G \mid c N A c \beta 1-3 G a l$ & 1 & 4 & 702 \\
\hline 14 & Blood group B antigen hexaose type 1 & Gal $\alpha 1-3$ (Fuc $\alpha 1-2$ )Gal $\beta 1-3$ GIcNAc $\beta 1-3 G a l \beta 1-4 G \mid c$ & -10 & 2 & -21 \\
\hline 15 & Blood group B antigen hexaose type 2 & Gal $\alpha 1-3$ (Fuc $\alpha 1-2)$ Gal $\beta 1-4$ GIcNAc $\beta 1-3 G a l \beta 1-4 G I c$ & -3 & 3 & -95 \\
\hline 16 & LewisX (LeX) tetraose & Gal $\beta 1-4$ ( Fuc $\alpha 1-3)$ GIcNAc $\beta 1-3 G a l$ & 8742 & 2417 & 28 \\
\hline 17 & LewisY (LeY) pentaose & Fuc $\alpha 1-2$ Gal $\beta 1-4$ (Fuc $\alpha 1-3)$ GIcNAc $\beta 1-3 G$ al & -3 & 5 & -182 \\
\hline 18 & Lewisa (Lea) tetraose & Gal $\beta 1-3$ (Fuc $\alpha 1-4$ )GIcNAc $\beta 1-3 G a l$ & 65066 & 24 & 0 \\
\hline 19 & Lewisb (Leb) pentaose & Fuc $\alpha 1-2$ Gal $\beta 1-3$ (Fuc $\alpha 1-4)$ GIcNAc $\beta 1-3 G a l$ & -2 & 6 & -272 \\
\hline 20 & Galili antigen pentaose & Gal $\alpha 1-3$ Gal $\beta 1-4 G \mid c N A c \beta 1-3$ Gal $\beta 1-4 G \mid c$ & 2 & 1 & 86 \\
\hline 21 & Isoglobopentaose / iGb5 & Gal $\beta 1-3 G a I N A c \beta 1-3 G a l \alpha 1-3 G a l \beta 1-4 G l c$ & 1 & 4 & 424 \\
\hline 22 & Galili antigen heptaose & Gal $\alpha 1-3($ Galß1-4GIcNAc $\beta 1-3) 2$ Galß1-4GIc & -5 & 7 & -128 \\
\hline 23 & Globotriaose (Gb3) / Pk antigen & 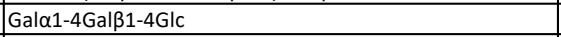 & 3 & 3 & 137 \\
\hline 24 & Globotetraose (Gb4) / P antigen & GalNAc $\beta 1-3$ Gal $\alpha 1-4$ Gal $\beta 1-4$ GIc & 1 & 2 & 416 \\
\hline 25 & Globo-H hexaose / Stage Specific Embryonic Antigen 3b (SSEA-3b) & Fuc $\alpha 1-2$ Gal $\beta 1-3 G$ alNAc $\beta 1-3 G a l \alpha 1-4 G a l \beta 1-4 G l c$ & 5 & 2 & 36 \\
\hline 26 & Globo-A heptaose & GalNAc $\alpha 1-3$ (Fuc $\alpha 1-2)$ Gal $\beta 1-3$ GalNAc $\beta 1-3 G$ Gal $\alpha 1-4$ Gal $\beta 1-4 G$ & 5 & 3 & 54 \\
\hline 27 & Globo-B heptaose & Gal $\alpha 1-3($ Fuc $\alpha 1-2)$ Gal $\beta 1-3 G a l N A c \beta 1-3 G a l \alpha 1-4 G a \mid \beta 1-4 G I c$ & 1 & 5 & 372 \\
\hline 28 & Blood group $\mathrm{H}$ antigen tetraose type 4 / Globo $\mathrm{H}$ tetraose & Fuc $\alpha 1-2$ Gal $\beta 1-3$ GalNAc $\beta 1-3 \mathrm{Gal}$ & 4 & 1 & 35 \\
\hline 29 & Blood group $\mathrm{A}$ antigen pentaose type 4 & 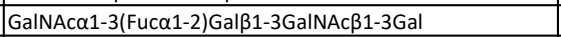 & 0 & 3 & 1052 \\
\hline 30 & Blood group B antigen pentasaccharide type 4 & 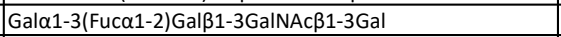 & 5 & 11 & 231 \\
\hline 31 & Forssman antigen pentaose & GalNAca1-3GalNAc $\beta 1-3 G a l \alpha 1-4 G a l \beta 1-4 G I c$ & 2 & 1 & 72 \\
\hline 32 & Isoforssman antigen pentaose & GaINAc $\alpha 1-3$ GalNAc $\beta 1-3 G a l \alpha 1-3 G a l \beta 1-4 G \mid c$ & 4 & 3 & 62 \\
\hline 33 & Globotriaose analogue type 2/ P1 antigen & Gal 1 1-4Galß1-4GIcNAc & -2 & 3 & -171 \\
\hline 34 & Isoglobo-H analogue type 1 & Fuc $\alpha 1-2$ Gal $\beta 1-3$ GIcNAc $\beta 1-3 G a l \alpha 1-3 G a l \beta 1-4 G \mid c$ & 5 & 3 & 69 \\
\hline 35 & OL-05 & GIcNAc $\beta 1-6(G l c N A c \beta 1-4)(G I c N A c \beta 1-2) M a n \alpha 1-6(G \mid c N A c \beta 1-$ & 4 & 3 & 86 \\
\hline 36 & OL-09 & Man $\alpha 1-6($ Man $\alpha 1-3)$ Man $\alpha 1-6($ GlcNAc $\beta 1-4)(G I c N A c \beta 1-4(G I c$ & 3 & 1 & 40 \\
\hline 37 & OL-13 & Gal $\beta 1-4 G I c N A c \beta 1-2$ Man $\alpha 1-6(G \mid c N A c \beta 1-4)(G a l \beta 1-4 G I c N A c \beta$ & 4 & 3 & 88 \\
\hline 38 & OL-17 & GIcNAc $\beta 1-4(G I c N A c \beta 1-2)$ Man $\alpha 1-6($ GIcNAc $\beta 1-4)(G I c N A c \beta 1-$ & 5 & 3 & 55 \\
\hline 39 & EY-10 & 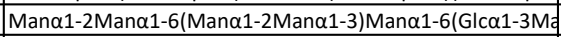 & -3 & 4 & -127 \\
\hline 40 & EY-15 & Man $\alpha 1-6($ Man $\alpha 1-3)$ Man $\alpha 1-6($ GlcNAc $\beta 1-4)(G I c N A c \beta 1-2 M a r$ & 0 & 4 & 1438 \\
\hline 41 & EY-16 & 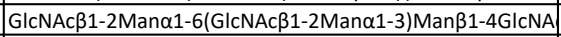 & 2 & 2 & 108 \\
\hline 42 & EY-26 & GIcNAcß1-2Man $\alpha 1-6($ GIcNAc $\beta 1-4)(G I c N A c \beta 1-2 M a n \alpha 1-3) M$ & 2 & 2 & 99 \\
\hline 43 & EY-32 & GlcNAc $\beta 1-6(G I c N A c \beta 1-4)($ GlcNAc $\beta 1-2) M a n \alpha 1-6(G \mid c N A c \beta 1-$ & -4 & 6 & -144 \\
\hline 44 & $2^{\prime}-\mathrm{FL}$ & Fuc $\alpha 1-2 \mathrm{Gal} \beta 1-4 \mathrm{Glc}$ & 8 & 12 & 161 \\
\hline 45 & 6'-SL & Neu5A $\alpha 2-6$ Gal $\beta 1-4 G \mid c$ & 3 & 1 & 46 \\
\hline 46 & EY-20 & $\begin{array}{l}\text { Neu5A } \alpha 2-6 \text { Gal } \beta 1-4 G \mid c N A c \beta 1-2 M a n \alpha 1-6(G \mid c N A c \beta 1- \\
2 \text { Man } \alpha 1-3) \text { Man } \beta 1-4 G I c N A c \beta 1-4 G I c N A c\end{array}$ & -1 & 1 & -258 \\
\hline 47 & Man8 & 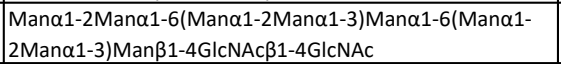 & -1 & 3 & -230 \\
\hline 48 & FBS-1 & 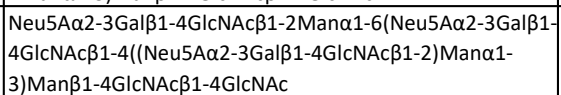 & 3 & 1 & 18 \\
\hline 49 & Water & & 1 & 2 & 200 \\
\hline 50 & Water & & 2 & 2 & 108 \\
\hline 51 & Water & & 2 & 3 & 164 \\
\hline 52 & Biotin-BSA & & 2 & 2 & 67 \\
\hline
\end{tabular}


Table S5. Printed microarray raw data. Column A to C list the ID, name and structure of printed glycans; Column D is the average relative fluorescent units (RFU); Column E is the standard deviation of 4 printed spots; Column $\mathrm{F}$ is the ratio between STDEV and average RFU.

\begin{tabular}{|c|c|c|c|c|c|}
\hline \multicolumn{6}{|c|}{ Anti-Le ${ }^{b}$ antibody $(1 / 100)$} \\
\hline Glycan ID & Glycan name & Structure & Average & STDEV & $\% \mathrm{CV}$ \\
\hline 1 & Lacto-N-tetraose (LNT) & Galß1-3GIcNac $\beta 1-3 G a|\beta 1-4 G| c$ & 0 & 2 & 825 \\
\hline 2 & Lacto-N-neotetraose (LNnT / neo-LNT) & Galß1-4GIcNAcß1-3Galß1-4GIc & 2 & 2 & 84 \\
\hline 3 & Blood group $\mathrm{H}$ antigen pentaose type 2 / Lacto-N-neofucopentaose I (LnNFP I) & Fuc $\alpha 1-2$ Gal $\beta 1-4 G \mid c N A c \beta 1-3 G a l \beta 1-4 G I c$ & 5 & 3 & 53 \\
\hline 4 & Blood group A Lewisb antigen pentaose type 1 & GalNAc $\alpha 1-3$ (Fuc $\alpha 1-2$ )Gal $\beta 1-3($ Fuc $\alpha 1-4)$ GIcNAc & 15 & 27 & 182 \\
\hline 5 & Blood group A LewisY antigen pentaose type 2 & GaINAc $\alpha 1-3$ (Fuc $\alpha 1-2$ )Galß1-4(Fuc $\alpha 1-3)$ GIcNAc & -1 & 3 & -441 \\
\hline 6 & Blood group $\mathrm{A}$ antigen pentaose type 1 & 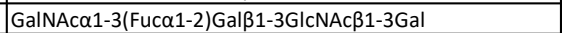 & 1 & 2 & 165 \\
\hline 7 & Blood group $\mathrm{A}$ antigen pentaose type 2 & GalNAca1-3(Fuc $\alpha 1-2)$ Galß1-4GIcNAc $\beta 1-3 G a l$ & 4 & 4 & 96 \\
\hline 8 & Blood group $A$ antigen hexaose type 1 & GalNAc $\alpha 1-3$ (Fuc $\alpha 1-2)$ Gal $\beta 1-3 G I c N A c \beta 1-3 G a l \beta 1-4 G \mid c$ & 4 & 3 & 68 \\
\hline 9 & Blood group $\mathrm{A}$ antigen hexaose type 2 & GalNAc $\alpha 1-3$ (Fuc $\alpha 1-2)$ Gal $\beta 1-4 G I c N A c \beta 1-3 G a l \beta 1-4 G I c$ & 6 & 1 & 23 \\
\hline 10 & Blood group B Lewisb antigen pentaose type 1 & Gal $\alpha 1-3$ (Fuc $\alpha 1-2$ )Gal $\beta 1-3$ (Fuc $\alpha 1-4)$ GIcNAc & 3 & 3 & 100 \\
\hline 11 & Blood group B LewisY antigen pentaose type 2 & Gal $\alpha 1-3$ (Fuc $\alpha 1-2)$ Galß1-4(Fuca1-3)GIcNAc & 0 & 5 & 1915 \\
\hline 12 & Blood group B antigen pentaose type 1 & 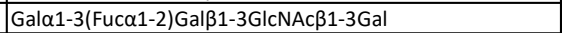 & 1 & 4 & 503 \\
\hline 13 & Blood group B antigen pentaose type 2 & Gal $\alpha 1-3$ (Fuc $\alpha 1-2$ )Gal $\beta 1-4 G I c N A c \beta 1-3 G a l$ & -1 & 2 & -177 \\
\hline 14 & Blood group B antigen hexaose type 1 & Gal $\alpha 1-3$ (Fuc $\alpha 1-2)$ Gal $\beta 1-3$ GIcNAc $\beta 1-3 G a l \beta 1-4 G \mid c$ & 1 & 4 & 302 \\
\hline 15 & Blood group $B$ antigen hexaose type 2 & Gal $\alpha 1-3$ (Fuc $\alpha 1-2)$ Gal $\beta 1-4 G|c N A c \beta 1-3 G a l \beta 1-4 G| c$ & -9 & 7 & -84 \\
\hline 16 & LewisX (LeX) tetraose & Galß1-4( Fuc $\alpha 1-3)$ GIcNAc $\beta 1-3 G a l$ & 15802 & 4526 & 29 \\
\hline 17 & LewisY (LeY) pentaose & Fuc $\alpha 1-2$ Gal $\beta 1-4$ (Fuc $\alpha 1-3)$ GIcNAc $\beta 1-3 G a l$ & 65015 & 564 & 1 \\
\hline 18 & Lewisa (Lea) tetraose & Galß1-3(Fuc $\alpha 1-4)$ GIcNAc $\beta 1-3 G a l$ & 64838 & 330 & 1 \\
\hline 19 & Lewisb (Leb) pentaose & Fuc $\alpha 1-2$ Gal $\beta 1-3($ Fuc $\alpha 1-4) G I c N A c \beta 1-3 G a l$ & 59753 & 869 & 1 \\
\hline 20 & Galili antigen pentaose & Gal $\alpha 1-3$ Gal $\beta 1-4 G|c N A c \beta 1-3 G a| \beta 1-4 G \mid c$ & 1 & 8 & 1046 \\
\hline 21 & Isoglobopentaose / iGb5 & 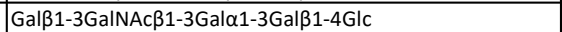 & 1 & 5 & 902 \\
\hline 22 & Galili antigen heptaose & 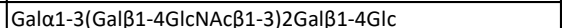 & -1 & 3 & -600 \\
\hline 23 & Globotriaose (Gb3) / Pk antigen & 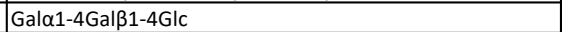 & -6 & 1 & -15 \\
\hline 24 & Globotetraose (Gb4) / P antigen & GalNAc $\beta 1-3$ Gal $\alpha 1-4$ Gal $\beta 1-4 G I c$ & 5 & 2 & 37 \\
\hline 25 & Globo-H hexaose / Stage Specific Embryonic Antigen 3b (SSEA-3b) & Fuc $\alpha 1-2$ Gal $\beta 1-3 G a l N A c \beta 1-3 G a l \alpha 1-4 G a l \beta 1-4 G \mid c$ & 15 & 16 & 113 \\
\hline 26 & Globo-A heptaose & 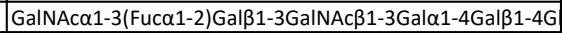 & 2 & 2 & 127 \\
\hline 27 & Globo-B heptaose & Gal $\alpha 1-3$ (Fuc $\alpha 1-2)$ Galß1-3GalNAc $\beta 1-3 G a l \alpha 1-4 G a l \beta 1-4 G \mid c$ & 0 & 4 & -1579 \\
\hline 28 & Blood group $\mathrm{H}$ antigen tetraose type 4 / Globo $\mathrm{H}$ tetraose & Fuc $\alpha 1-2$ Galß1-3GalNAc $\beta 1-3 G a l$ & 3 & 2 & 72 \\
\hline 29 & Blood group $\mathrm{A}$ antigen pentaose type 4 & 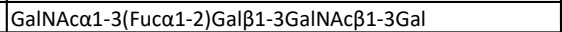 & 3 & 1 & 46 \\
\hline 30 & Blood group B antigen pentasaccharide type 4 & Gal $\alpha 1-3$ (Fuc $\alpha 1-2$ )Galß1-3GalNAc $\beta 1-3 G a l$ & 0 & 5 & 1829 \\
\hline 31 & Forssman antigen pentaose & GalNAc $\alpha 1-3$ GalNAc $\beta 1-3 G a l \alpha 1-4 G a l \beta 1-4 G \mid c$ & -6 & 11 & -209 \\
\hline 32 & Isoforssman antigen pentaose & GaINAc $\alpha 1-3$ GalNAc $\beta 1-3 G a l \alpha 1-3 G a l \beta 1-4 G \mid c$ & -1 & 3 & -529 \\
\hline 33 & Globotriaose analogue type 2/ P1 antigen & Gal 1 1-4Galß1-4GIcNAc & 4 & 2 & 52 \\
\hline 34 & Isoglobo- $\mathrm{H}$ analogue type 1 & Fuc $\alpha 1-2$ Gal $\beta 1-3 \mathrm{GlcNAc} \beta 1-3 \mathrm{Gal} \alpha 1-3 \mathrm{Gal} \beta 1-4 \mathrm{Glc}$ & 7 & 6 & 88 \\
\hline 35 & OL-05 & GIcNAc $\beta 1-6($ GIcNAc $\beta 1-4)(G I c N A c \beta 1-2)$ Man $\alpha 1-6($ GIcNAc $\beta 1-$ & 2 & 2 & 99 \\
\hline 36 & OL-09 & Man $\alpha 1-6($ Man $\alpha 1-3)$ Man $\alpha 1-6($ GlcNAc $\beta 1-4)(G I c N A c \beta 1-4(G I c$ & 4 & 5 & 124 \\
\hline 37 & $\mathrm{OL}-13$ & Gal $\beta 1-4 G I c N A c \beta 1-2 M a n \alpha 1-6(G I c N A c \beta 1-4)(G a l \beta 1-4 G I c N A c \beta$ & 0 & 5 & \#DIV/0! \\
\hline 38 & OL-17 & GIcNAc $\beta 1-4(G I c N A c \beta 1-2) M a n \alpha 1-6(G \mid c N A c \beta 1-4)(G \mid c N A c \beta 1-$ & 2 & 6 & 316 \\
\hline 39 & EY-10 & 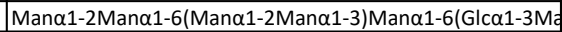 & 0 & 3 & 1000 \\
\hline 40 & EY-15 & Man $\alpha 1-6($ Man $\alpha 1-3)$ Man $\alpha 1-6($ GIcNAc $\beta 1-4)(G I c N A c \beta 1-2 M a r$ & 27 & 49 & 178 \\
\hline 41 & EY-16 & GIcNAc $\beta 1-2$ Man $\alpha 1-6($ GIcNAc $\beta 1-2 M a n \alpha 1-3)$ Man $\beta 1-4 G I c N A$ & -3 & 6 & -185 \\
\hline 42 & EY-26 & GIcNAc $\beta 1-2 M a n \alpha 1-6(G \mid c N A c \beta 1-4)(G I c N A c \beta 1-2 M a n \alpha 1-3) M$ & 3 & 4 & 152 \\
\hline 43 & EY-32 & GIcNAc $\beta 1-6($ GIcNAc $\beta 1-4)(G \mid c N A c \beta 1-2)$ Man $\alpha 1-6($ GIcNAc $\beta 1-$ & 3 & 3 & 101 \\
\hline 44 & $2^{\prime}-\mathrm{FL}$ & Fuc $\alpha 1-2$ Gal $\beta 1-4 G l c$ & 6 & 1 & 23 \\
\hline 45 & 6'-SL & 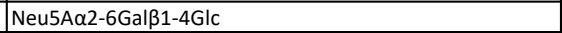 & 1 & 2 & 151 \\
\hline 46 & EY-20 & 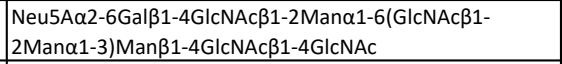 & -1 & 3 & -367 \\
\hline 47 & Man8 & 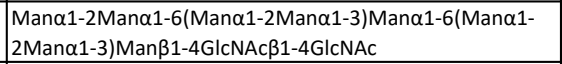 & 2 & 1 & 22 \\
\hline 48 & FBS-1 & 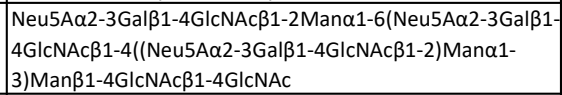 & 5 & 4 & 71 \\
\hline 49 & Water & & -1 & 5 & -389 \\
\hline 50 & Water & & 1 & 4 & 424 \\
\hline 51 & Water & & -1 & 4 & -702 \\
\hline 52 & Biotin-BSA & & 3 & 1 & 46 \\
\hline
\end{tabular}


Table S5. Printed microarray raw data. Column A to C list the ID, name and structure of printed glycans; Column D is the average relative fluorescent units (RFU); Column E is the standard deviation of 4 printed spots; Column $\mathrm{F}$ is the ratio between STDEV and average RFU.

\begin{tabular}{|c|c|c|c|c|c|}
\hline \multicolumn{6}{|c|}{ Anti-Le ${ }^{a}$ antibody $(1 / 1000)$} \\
\hline Glycan ID & Glycan name & Structure & Average & STDEV & $\% \mathrm{CV}$ \\
\hline 1 & Lacto-N-tetraose (LNT) & Galß1-3GIcNac $\beta 1-3 G a l \beta 1-4 G \mid c$ & 2 & 5 & 257 \\
\hline 2 & Lacto-N-neotetraose (LNnT / neo-LNT) & Galß1-4GIcNAcß1-3Galß1-4GIc & 0 & 1 & -383 \\
\hline 3 & Blood group $\mathrm{H}$ antigen pentaose type 2 / Lacto-N-neofucopentaose I (LnNFP I) & Fuc $\alpha 1-2$ Gal $\beta 1-4$ GIcNAc $\beta 1-3 G a l \beta 1-4 G l c$ & 5 & 3 & 53 \\
\hline 4 & Blood group A Lewisb antigen pentaose type 1 & GalNAc $\alpha 1-3$ (Fuc $\alpha 1-2)$ Gal $\beta 1-3$ (Fuc $\alpha 1-4)$ GIcNAc & 2 & 4 & 280 \\
\hline 5 & Blood group A LewisY antigen pentaose type 2 & GalNAc $\alpha 1-3$ (Fuc $\alpha 1-2)$ Gal $\beta 1-4($ Fuc $\alpha 1-3)$ GIcNAc & 2 & 2 & 92 \\
\hline 6 & Blood group A antigen pentaose type 1 & GalNAc $\alpha 1-3$ (Fuc $\alpha 1-2)$ Gal $\beta 1-3$ GIcNAc $\beta 1-3 G a l$ & 1 & 2 & 120 \\
\hline 7 & Blood group $\mathrm{A}$ antigen pentaose type 2 & GalNAc $\alpha 1-3$ (Fuc $\alpha 1-2)$ Gal $\beta 1-4$ GIcNAc $\beta 1-3 G a l$ & 4 & 3 & 74 \\
\hline 8 & Blood group $A$ antigen hexaose type 1 & GalNAca1-3(Fuc $\alpha 1-2$ )Gal $\beta 1-3 G I c N A c \beta 1-3 G a l \beta 1-4 G \mid c$ & 3 & 6 & 232 \\
\hline 9 & Blood group $A$ antigen hexaose type 2 & GalNAca1-3(Fuc $\alpha 1-2$ )Galß1-4GIcNAc $\beta 1-3 G a l \beta 1-4 G \mid c$ & 6 & 3 & 47 \\
\hline 10 & Blood group B Lewisb antigen pentaose type 1 & Gal $\alpha 1-3$ (Fuc $\alpha 1-2$ )Galß1-3(Fuc $\alpha 1-4)$ GlcNAc & 2 & 1 & 86 \\
\hline 11 & Blood group B LewisY antigen pentaose type 2 & Gal $\alpha 1-3$ (Fuc $\alpha 1-2)$ Galß1-4(Fuc $\alpha 1-3)$ GlcNAc & 3 & 3 & 106 \\
\hline 12 & Blood group B antigen pentaose type 1 & Gal $\alpha 1-3($ Fuc $\alpha 1-2)$ Gal $\beta 1-3 G \mid c N A c \beta 1-3 G a l$ & 1 & 6 & 1194 \\
\hline 13 & Blood group B antigen pentaose type 2 & Gal $\alpha 1-3($ Fuc $\alpha 1-2)$ Gal $\beta 1-4 G \mid c N A c \beta 1-3 G a l$ & 1 & 1 & 258 \\
\hline 14 & Blood group B antigen hexaose type 1 & Gal $\alpha 1-3$ (Fuc $\alpha 1-2)$ Gal $\beta 1-3$ GIcNAc $\beta 1-3 G a l \beta 1-4 G l c$ & -1 & 4 & -365 \\
\hline 15 & Blood group B antigen hexaose type 2 & Gal $\alpha 1-3$ (Fuc $\alpha 1-2)$ Gal $\beta 1-4$ GIcNAc $\beta 1-3 G a \mid \beta 1-4 G I c$ & 6 & 5 & 82 \\
\hline 16 & LewisX (LeX) tetraose & Gal $\beta 1-4$ ( Fuc $\alpha 1-3)$ GlcNAc $\beta 1-3 G a l$ & 404 & 110 & 27 \\
\hline 17 & LewisY (LeY) pentaose & Fuc $\alpha 1-2$ Gal $\beta 1-4($ Fuc $\alpha 1-3)$ GlcNAc $\beta 1-3 G$ al & -2 & 3 & -176 \\
\hline 18 & Lewisa (Lea) tetraose & Gal $\beta 1-3$ (Fuc $\alpha 1-4$ )GIcNAc $\beta 1-3 G a l$ & 21055 & 645 & 3 \\
\hline 19 & Lewisb (Leb) pentaose & Fuc $\alpha 1-2$ Gal $\beta 1-3($ Fuc $\alpha 1-4)$ GlcNAc $\beta 1-3 G a l$ & 3 & 1 & 47 \\
\hline 20 & Galili antigen pentaose & Gal $\alpha 1-3$ Gal $\beta 1-4 G I c N A c \beta 1-3 G a l \beta 1-4 G \mid c$ & -3 & 8 & -334 \\
\hline 21 & Isoglobopentaose / iGb5 & Gal $\beta 1-3 G a I N A c \beta 1-3 G a l \alpha 1-3 G a l \beta 1-4 G \mid c$ & 2 & 2 & 84 \\
\hline 22 & Galili antigen heptaose & Gal $\alpha 1-3($ Galß1-4GIcNAc $\beta 1-3) 2$ Galß1-4Glc & 5 & 2 & 29 \\
\hline 23 & Globotriaose (Gb3) / Pk antigen & Gala1-4Galß1-4GIc & 4 & 4 & 89 \\
\hline 24 & Globotetraose (Gb4) / P antigen & GalNAc $\beta 1-3 G a l \alpha 1-4 G a \mid \beta 1-4 G I c$ & 3 & 2 & 72 \\
\hline 25 & Globo-H hexaose / Stage Specific Embryonic Antigen 3b (SSEA-3b) & Fuc $\alpha 1-2$ Gal $\beta 1-3 G a l N A c \beta 1-3 G a l \alpha 1-4 G a l \beta 1-4 G l c$ & 3 & 3 & 105 \\
\hline 26 & Globo-A heptaose & GalNAc $\alpha 1-3$ (Fuc $\alpha 1-2)$ Gal $\beta 1-3 G a l N A c \beta 1-3 G a l \alpha 1-4 G a l \beta 1-4 G$ & 5 & 5 & 104 \\
\hline 27 & Globo-B heptaose & Gal $\alpha 1-3($ Fuc $\alpha 1-2)$ Gal $\beta 1-3 G a l N A c \beta 1-3 G a l \alpha 1-4 G a \mid \beta 1-4 G l c$ & -8 & 12 & -162 \\
\hline 28 & Blood group $\mathrm{H}$ antigen tetraose type 4 / Globo $\mathrm{H}$ tetraose & Fuc $\alpha 1-2$ Gal $\beta 1-3 G$ alNAc $\beta 1-3 G$ al & 0 & 1 & 503 \\
\hline 29 & Blood group $\mathrm{A}$ antigen pentaose type 4 & GalNAc $\alpha 1-3$ (Fuc $\alpha 1-2)$ Gal $\beta 1-3 G a l N A c \beta 1-3 G a l$ & 1 & 1 & 128 \\
\hline 30 & Blood group B antigen pentasaccharide type 4 & 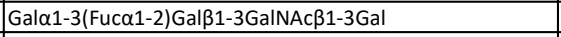 & 3 & 3 & 99 \\
\hline 31 & Forssman antigen pentaose & GalNAc $\alpha 1-3$ GalNAc $\beta 1-3 G a l \alpha 1-4 G a l \beta 1-4 G \mid c$ & -6 & 11 & -184 \\
\hline 32 & Isoforssman antigen pentaose & GalNAc $\alpha 1-3 G a I N A c \beta 1-3 G a l \alpha 1-3 G a|\beta 1-4 G| c$ & 3 & 2 & 81 \\
\hline 33 & Globotriaose analogue type 2/ P1 antigen & Gal $\alpha 1-4$ Galß1-4GIcNAc & 3 & 2 & 67 \\
\hline 34 & Isoglobo- $\mathrm{H}$ analogue type 1 & Fuc $\alpha 1-2$ Gal $\beta 1-3 G \mid c N A c \beta 1-3 G a l \alpha 1-3 G a l \beta 1-4 G I c$ & 8 & 6 & 80 \\
\hline 35 & OL-05 & GIcNAc $\beta 1-6(G \mid c N A c \beta 1-4)(G \mid c N A c \beta 1-2) M a n \alpha 1-6(G \mid c N A c \beta 1-$ & 1 & 2 & 296 \\
\hline 36 & OL-09 & Man $\alpha 1-6($ Man $\alpha 1-3)$ Man $\alpha 1-6($ GlcNAc $\beta 1-4)(G I c N A c \beta 1-4(G I C$ & 2 & 3 & 221 \\
\hline 37 & OL-13 & Galß1-4GIcNAc $\beta 1-2 M a n \alpha 1-6(G \mid c N A c \beta 1-4)(G a|\beta 1-4 G| c N A c \beta$ & 0 & 4 & \#DIV/0! \\
\hline 38 & OL-17 & GIcNAc $\beta 1-4(G \mid c N A c \beta 1-2) M a n \alpha 1-6(G I c N A c \beta 1-4)(G \mid c N A c \beta 1-$ & 0 & 2 & \#DIV/0! \\
\hline 39 & EY-10 & Man $\alpha 1-2 M a n \alpha 1-6($ Man $\alpha 1-2 M a n \alpha 1-3)$ Man $\alpha 1-6(G \mid c \alpha 1-3 M a$ & 2 & 1 & 86 \\
\hline 40 & EY-15 & Man $\alpha 1-6($ Man $\alpha 1-3)$ Man $\alpha 1-6($ GlcNAc $\beta 1-4)($ GIcNAc $\beta 1-2 M a r$ & 3 & 3 & 104 \\
\hline 41 & EY-16 & GIcNAc $\beta 1-2 M a n \alpha 1-6(G \mid c N A c \beta 1-2 M a n \alpha 1-3) M a n \beta 1-4 G I c N A$ & -1 & 3 & -367 \\
\hline 42 & EY-26 & GIcNAc $\beta 1-2 M a n \alpha 1-6($ GlcNAc $\beta 1-4)(G I c N A c \beta 1-2 M a n \alpha 1-3) M$ & 6 & 6 & 101 \\
\hline 43 & EY-32 & GlcNAc $\beta 1-6(G l c N A c \beta 1-4)($ GlcNAc $\beta 1-2) M a n \alpha 1-6(G \mid c N A c \beta 1-$ & -179 & 364 & -203 \\
\hline 44 & 2'-FL & Fuca1-2Galß1-4Glc & 2 & 4 & 204 \\
\hline 45 & 6'-SL & Neu5A $\alpha 2-6$ Gal $\beta 1-4 G$ lc & 4 & 4 & 106 \\
\hline 46 & EY-20 & 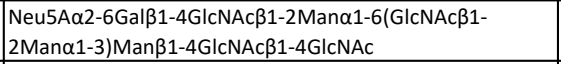 & 3 & 1 & 52 \\
\hline 47 & Man8 & $\begin{array}{l}\text { Man } \alpha 1-2 \text { Man } \alpha 1-6(\text { Man } \alpha 1-2 \text { Man } \alpha 1-3) \text { Man } \alpha 1-6(\text { Man } \alpha 1- \\
2 \text { Man } 1-3) \text { Man } \beta 1-4 G I c N A c \beta 1-4 G I c N A c\end{array}$ & -48 & 527 & -1099 \\
\hline 48 & FBS-1 & $\begin{array}{l}\text { Neu5A } \alpha 2-3 \text { Gal } \beta 1-4 G I c N A c \beta 1-2 \text { Man } \alpha 1-6(\text { Neu5A } \alpha 2-3 G a l \beta 1- \\
\text { 4GIcNAc } \beta 1-4((\text { Neu5A } \alpha 2-3 G a l \beta 1-4 G I c N A c \beta 1-2) \text { Man } \alpha 1- \\
\text { 3)Man } \beta 1-4 G I c N A c \beta 1-4 G I c N A c\end{array}$ & 5 & 6 & 134 \\
\hline 49 & Water & & 4 & 5 & 114 \\
\hline 50 & Water & & 0 & 1 & \#DIV/0! \\
\hline 51 & Water & & 2 & 4 & 221 \\
\hline 52 & Biotin-BSA & & 1 & 2 & 151 \\
\hline
\end{tabular}


Table S5. Printed microarray raw data. Column A to C list the ID, name and structure of printed glycans; Column D is the average relative fluorescent units (RFU); Column E is the standard deviation of 4 printed spots; Column $\mathrm{F}$ is the ratio between STDEV and average RFU.

\begin{tabular}{|c|c|c|c|c|c|}
\hline \multicolumn{6}{|c|}{ Anti-Le ${ }^{b}$ antibody $(1 / 1000)$} \\
\hline Glycan ID & Glycan name & Structure & Average & STDEV & $\% \mathrm{CV}$ \\
\hline 1 & Lacto-N-tetraose (LNT) & Galß1-3GIcNacß1-3Galß1-4Glc & 1 & 3 & 622 \\
\hline 2 & Lacto-N-neotetraose (LNnT / neo-LNT) & 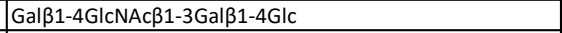 & 5 & 2 & 36 \\
\hline 3 & Blood group $\mathrm{H}$ antigen pentaose type 2 / Lacto-N-neofucopentaose I (LnNFP I) & Fuc $\alpha 1-2$ Gal $\beta 1-4$ GIcNAc $\beta 1-3 G a l \beta 1-4 G I c$ & 7 & 6 & 88 \\
\hline 4 & Blood group A Lewisb antigen pentaose type 1 & GalNAc $\alpha 1-3$ (Fuc $\alpha 1-2$ )Galß1-3(Fuc $\alpha 1-4)$ GIcNAc & 6 & 5 & 83 \\
\hline 5 & Blood group A LewisY antigen pentaose type 2 & GalNAc $\alpha 1-3$ (Fuc $\alpha 1-2$ )Galß1-4(Fuc $\alpha 1-3)$ GIcNAc & 0 & 4 & 1510 \\
\hline 6 & Blood group $A$ antigen pentaose type 1 & GalNAc $\alpha 1-3$ (Fuc $\alpha 1-2)$ Gal $\beta 1-3$ GIcNAc $\beta 1-3 G a l$ & 3 & 6 & 182 \\
\hline 7 & Blood group $\mathrm{A}$ antigen pentaose type 2 & 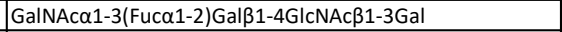 & 7 & 1 & 20 \\
\hline 8 & Blood group A antigen hexaose type 1 & GalNAc $\alpha 1-3$ (Fuc $\alpha 1-2)$ Gal $\beta 1-3 G I c N A c \beta 1-3 G a l \beta 1-4 G I c$ & 4 & 4 & 104 \\
\hline 9 & Blood group $A$ antigen hexaose type 2 & GalNAc $\alpha 1-3$ (Fuc $\alpha 1-2)$ Gal $\beta 1-4$ GIcNAc $\beta 1-3 G a l \beta 1-4 G I c$ & 5 & 7 & 139 \\
\hline 10 & Blood group B Lewisb antigen pentaose type 1 & Gal $\alpha 1-3$ (Fuc $\alpha 1-2$ )Gal $\beta 1-3$ (Fuc $\alpha 1-4)$ GlcNAc & 0 & 3 & -1102 \\
\hline 11 & Blood group B LewisY antigen pentaose type 2 & Gal 1 1-3(Fuc $\alpha 1-2$ )Galß1-4(Fuca1-3)GIcNAc & 6 & 5 & 74 \\
\hline 12 & Blood group B antigen pentaose type 1 & Gal $\alpha 1-3$ (Fuc $\alpha 1-2$ )Gal $\beta 1-3 G \mid c N A c \beta 1-3 G a l$ & 1 & 2 & 383 \\
\hline 13 & Blood group B antigen pentaose type 2 & Gal $\alpha 1-3$ (Fuc $\alpha 1-2$ )Galß1-4GIcNAc $\beta 1-3 G a l$ & 3 & 1 & 52 \\
\hline 14 & Blood group B antigen hexaose type 1 & Gal $\alpha 1-3$ (Fuc $\alpha 1-2)$ Gal $\beta 1-3$ GlcNAc $\beta 1-3 G a l \beta 1-4 G \mid c$ & 3 & 4 & 124 \\
\hline 15 & Blood group B antigen hexaose type 2 & Gal $\alpha 1-3$ (Fuc $\alpha 1-2)$ Gal $\beta 1-4$ GlcNAc $\beta 1-3 G a \mid \beta 1-4 G l c$ & 6 & 5 & 85 \\
\hline 16 & LewisX (LeX) tetraose & Gal $\beta 1-4$ ( Fuc $\alpha 1-3)$ GIcNAc $\beta 1-3 G a l$ & 738 & 110 & 15 \\
\hline 17 & LewisY (LeY) pentaose & 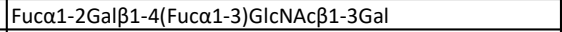 & 15044 & 1584 & 11 \\
\hline 18 & Lewisa (Lea) tetraose & Galß1-3(Fuc $\alpha 1-4)$ GlcNAc $\beta 1-3 G a l$ & 16293 & 312 & 2 \\
\hline 19 & Lewisb (Leb) pentaose & Fuc $\alpha 1-2$ Gal $\beta 1-3$ (Fuc $\alpha 1-4) G I c N A c \beta 1-3 G a l$ & 18061 & 1104 & 6 \\
\hline 20 & Galili antigen pentaose & Gal $\alpha 1-3$ Gal $\beta 1-4 G|c N A c \beta 1-3 G a l \beta 1-4 G| c$ & 6 & 5 & 83 \\
\hline 21 & Isoglobopentaose / iGb5 & Gal $\beta 1-3 G a l N A c \beta 1-3 G a l \alpha 1-3 G a l \beta 1-4 G l c$ & 2 & 4 & 246 \\
\hline 22 & Galili antigen heptaose & Gal $\alpha 1-3($ Gal $\beta 1-4 G I c N A c \beta 1-3) 2$ Gal $\beta 1-4 G \mid c$ & 2 & 4 & 246 \\
\hline 23 & Globotriaose (Gb3) / Pk antigen & Gala1-4Galß1-4Glc & 4 & 3 & 68 \\
\hline 24 & Globotetraose (Gb4) / $P$ antigen & GalNAc $\beta 1-3$ Gal $\alpha 1-4$ Gal$\beta 1-4 G l c$ & 2 & 4 & 205 \\
\hline 25 & Globo-H hexaose / Stage Specific Embryonic Antigen 3b (SSEA-3b) & Fuc $\alpha 1-2$ Gal $\beta 1-3 G$ alNAc $\beta 1-3 G a l \alpha 1-4 G a l \beta 1-4 G l c$ & 7 & 4 & 54 \\
\hline 26 & Globo-A heptaose & GalNAc $\alpha 1-3($ Fuc $\alpha 1-2)$ Gal $\beta 1-3 G a l N A c \beta 1-3 G a l \alpha 1-4 G a l \beta 1-4 G$ & 2 & 3 & 158 \\
\hline 27 & Globo-B heptaose & Gal $\alpha 1-3$ (Fuc $\alpha 1-2)$ Galß1-3GalNAc $\beta 1-3 G a l \alpha 1-4 G a l \beta 1-4 G l c$ & 4 & 2 & 40 \\
\hline 28 & Blood group $\mathrm{H}$ antigen tetraose type 4 / Globo $\mathrm{H}$ tetraose & Fuca1-2Gal $\beta 1-3 G a l N A c \beta 1-3 G a l$ & 7 & 4 & 48 \\
\hline 29 & Blood group A antigen pentaose type 4 & GalNAc $\alpha 1-3($ Fuc $\alpha 1-2)$ Gal $\beta 1-3$ GalNAc $\beta 1-3 G a l$ & 4 & 4 & 82 \\
\hline 30 & Blood group B antigen pentasaccharide type 4 & Gal $\alpha 1-3$ (Fuc $\alpha 1-2$ )Gal $\beta 1-3 G a l N A c \beta 1-3 G a l$ & 1 & 2 & 228 \\
\hline 31 & Forssman antigen pentaose & GalNAc $\alpha 1-3$ GalNAc $\beta 1-3 G a l \alpha 1-4 G a l \beta 1-4 G l c$ & 1 & 2 & 228 \\
\hline 32 & Isoforssman antigen pentaose & GaINAc $\alpha 1-3$ GalNAc $\beta 1-3 G a l \alpha 1-3 G a l \beta 1-4 G l c$ & 8 & 3 & 38 \\
\hline 33 & Globotriaose analogue type 2/ P1 antigen & Gal $\alpha 1-4$ Galß1-4GIcNAc & 7 & 4 & 51 \\
\hline 34 & Isoglobo- $\mathrm{H}$ analogue type 1 & Fuc $\alpha 1-2$ Gal $\beta 1-3 G I c N A c \beta 1-3 G a l \alpha 1-3 G a l \beta 1-4 G \mid c$ & 7 & 6 & 99 \\
\hline 35 & OL-05 & GlcNAc $\beta 1-6(G I c N A c \beta 1-4)(G \mid c N A c \beta 1-2) M a n \alpha 1-6(G \mid c N A c \beta 1-$ & 4 & 3 & 89 \\
\hline 36 & OL-09 & Man $\alpha 1-6($ Man $\alpha 1-3)$ Man $\alpha 1-6(G l c N A c \beta 1-4)(G l c N A c \beta 1-4(G I C$ & 3 & 4 & 155 \\
\hline 37 & $\mathrm{OL}-13$ & Gal $\beta 1-4$ GIcNAc $\beta 1-2 M a n \alpha 1-6($ GlcNAc $\beta 1-4)(G a l \beta 1-4 G I c N A c \beta$ & 3 & 1 & 29 \\
\hline 38 & OL-17 & GlcNAc $\beta 1-4(G I c N A c \beta 1-2) M a n \alpha 1-6(G l c N A c \beta 1-4)(G I c N A c \beta 1-$ & 2 & 3 & 138 \\
\hline 39 & EY-10 & 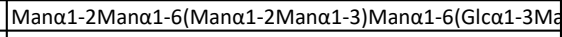 & 2 & 6 & 289 \\
\hline 40 & EY-15 & Man $\alpha 1-6($ Man $\alpha 1-3)$ Man $\alpha 1-6($ GlcNAc $\beta 1-4)(G l c N A c \beta 1-2 M a r$ & 0 & 2 & 887 \\
\hline 41 & EY-16 & GlcNAc $\beta 1-2$ Man $\alpha 1-6($ GlcNAc $\beta 1-2 M a n \alpha 1-3)$ Man $\beta 1-4 G I c N A$ & 2 & 6 & 356 \\
\hline 42 & EY-26 & GlcNAc $\beta 1-2$ Man $\alpha 1-6($ GlcNAc $\beta 1-4)(G I c N A c \beta 1-2 M a n \alpha 1-3) M$ & -2 & 4 & -204 \\
\hline 43 & EY-32 & GlcNAc $\beta 1-6(G I c N A c \beta 1-4)(G l c N A c \beta 1-2) M a n \alpha 1-6(G \mid c N A c \beta 1-$ & 2 & 5 & 265 \\
\hline 44 & 2'-FL & Fuc $\alpha 1-2 \mathrm{Gal} \beta 1-4 \mathrm{Glc}$ & 6 & 5 & 91 \\
\hline 45 & 6'-SL & 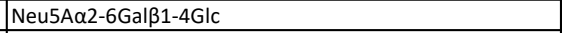 & 6 & 2 & 33 \\
\hline 46 & EY-20 & 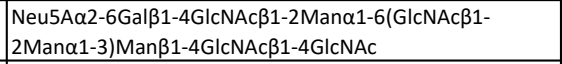 & 0 & 2 & -600 \\
\hline 47 & Man8 & 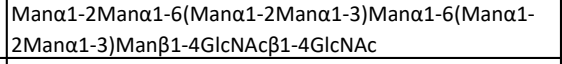 & 2 & 5 & 257 \\
\hline 48 & FBS-1 & $\begin{array}{l}\text { Neu5A } \alpha 2-3 \text { Gal } \beta 1-4 G I c N A c \beta 1-2 \text { Man } \alpha 1-6 \text { (Neu5A } \alpha 2-3 G a l \beta 1- \\
\text { 4GIcNAc } \beta 1-4((N e u 5 A \alpha 2-3 G a l \beta 1-4 G I c N A c \beta 1-2) \text { Man } \alpha 1- \\
\text { 3)Man } \beta 1-4 G I c N A c \beta 1-4 G I c N A c\end{array}$ & 6 & 5 & 97 \\
\hline 49 & Water & & 1 & 2 & 216 \\
\hline 50 & Water & & -1 & 7 & -1332 \\
\hline 51 & Water & & 3 & 5 & 210 \\
\hline 52 & Biotin-BSA & & -3 & 6 & -220 \\
\hline
\end{tabular}


Table S6. Standard curve for quantification of AEAB-labelled glycans. Use LNnT-AEAB as a standard. Column $A$ is the amount of LNnT-AEAB; Column B is the corresponding UV absorption at $330 \mathrm{~nm}$. The equation from linear fitting is shown in the chart.

\begin{tabular}{|r|r|}
\hline $\begin{array}{c}\text { LNnT- } \\
\text { AEAB } \\
\text { (nmol) }\end{array}$ & $\begin{array}{c}\text { UV absorption } \\
\mathbf{3 3 0} \mathbf{~ n m}\end{array}$ \\
\hline 0.2 & 34430 \\
\hline 0.8 & 135273 \\
\hline 2 & 338234 \\
\hline 5 & 846618 \\
\hline
\end{tabular}

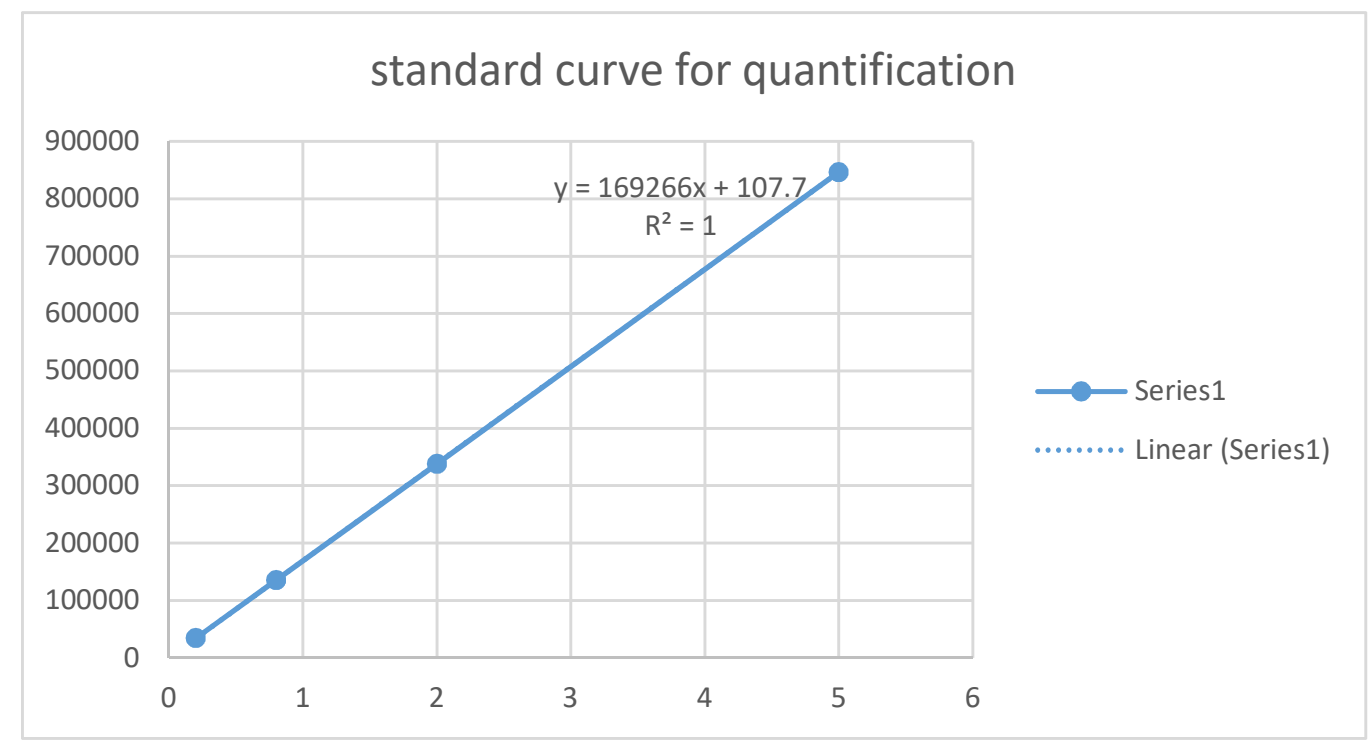


Table S6. Information of glycan fractions separated from pig kidneys. Column A and B represent the ID and name of the glycan fractions; Column C and D record the retention times of the corresponding fractions on NH2-HPLC and PGC-HPLC; Column E records the UV absorption of the corresponding fraction at $330 \mathrm{~nm}$; Column F shows the total amount of glycans in the corresponding fraction calculated according to the equation from the standard curve; Column $\mathrm{G}$ to $\mathrm{K}$ shows the molecular weight of the AEAB-labelled glycans in corresponding fraction on MALDI-TOF-MS with positive mode and negative mode, respectively; Column $\mathrm{K}$ shows the predicted glycan composition of the major glycan in

corresponding fraction; column L shows the DNA code for glycan fraction coding; Column M shows which subarray the DNA-coded glycan fraction belong to. Note: N.D. means Not Determined.

\begin{tabular}{|c|c|c|c|c|c|c|c|c|c|c|c|c|}
\hline \multirow[b]{3}{*}{$\begin{array}{l}\text { Fraction } \\
\text { ID }\end{array}$} & \multirow[b]{3}{*}{ Name } & \multirow[b]{3}{*}{$\begin{array}{c}\text { Retention time } \\
\text { (range) on } \\
\text { amine column } \\
\text { (min) }\end{array}$} & \multirow[b]{3}{*}{\begin{tabular}{|c} 
Retention \\
time (peak) \\
on PGC \\
column \\
(min)
\end{tabular}} & \multirow[b]{3}{*}{\begin{tabular}{|c|} 
UV \\
absorption \\
$330 \mathrm{~nm}$
\end{tabular}} & \multirow[b]{3}{*}{$\begin{array}{c}\text { total } \\
\text { amount } \\
\text { (nmol) }\end{array}$} & \multicolumn{4}{|c|}{ Molecular weight from MALDI-TOF MS (Da) } & & & \\
\hline & & & & & & Positive & mode & Negat & ive mode & & & \\
\hline & & & & & & $\begin{array}{l}\text { Major } \\
\text { peak }\end{array}$ & $\begin{array}{c}\text { Minor } \\
\text { peak }\end{array}$ & $\begin{array}{c}\text { Major } \\
\text { peak }\end{array}$ & Minor peak & $\begin{array}{c}\text { Composition of major } \\
\text { peak }\end{array}$ & DNA code & Subarray \\
\hline 1 & pig2 kidney-NH2-F1-PGC-F1 & $36 \sim 38$ & 22.4 & 8041 & 4.6 & 1096.33 & & & & $\mathrm{H} 3 \mathrm{~N} 2$ & 1 & \\
\hline 2 & pig2 kidney-NH2-F2-PGC-F1 & $38 \sim 39$ & $21.4+21.9$ & 14783 & 8.6 & 1258.22 & & & & $\mathrm{H} 4 \mathrm{~N} 2$ & 2 & \\
\hline 3 & pig2 kidney-NH2-F2-PGC-F2 & $38 \sim 39$ & 23.2 & 31406 & 18.3 & 1242.6 & 1118.1 & & & H3N2F1 & 3 & \\
\hline 4 & pig2 kidney-NH2-F3-PGC-F1 & $39 \sim 41$ & 17.9 & 22825 & 13.3 & 1299.3 & & & & H3N3 & 4 & \\
\hline 5 & pig2 kidney-NH2-F3-PGC-F2 & $39 \sim 41$ & 20.1 & 35989 & 21.0 & 1217.5 & & & & H5N1 & 5 & \\
\hline 6 & pig2 kidney-NH2-F4-PGC-F1 & $41 \sim 42.5$ & 19.8 & 29110 & 17.0 & 1502.6 & 1329.8 & & & H3N4 & 6 & \\
\hline 7 & pig2 kidney-NH2-F4-PGC-F2 & $41^{\sim} 42.5$ & 21.2 & 165212 & 96.6 & 1420.7 & 1098.5 & & & $\mathrm{H} 5 \mathrm{~N} 2$ & 7 & \\
\hline 8 & pig2 kidney-NH2-F5-PGC-F1 & $42.5^{\sim} 43.4$ & 19.5 & 25437 & 14.8 & 1706.1 & 1420.77 & & & H3N5 & 8 & \\
\hline 9 & pig2 kidney-NH2-F5-PGC-F2 & $42.5 \sim 43.4$ & 20.6 & 81554 & 47.6 & 1648.7 & & & & H3N4F1 & 9 & \\
\hline 10 & pig2 kidney-NH2-F6-PGC-F1 & $43.4 \sim 44.3$ & 17.6 & 16924 & 9.8 & 1379.9 & & & & H6N1 & 10 & \\
\hline 11 & pig2 kidney-NH2-F6-PGC-F2 & $43.4 \sim 44.3$ & 19.5 & 17524 & 10.2 & 1909.7 & 1735.996 & & & H3N6 & 11 & \\
\hline 12 & pig2 kidney-NH2-F6-PGC-F3 & $43.4 \sim 44.3$ & $19.5+19.7$ & 31581 & 18.4 & 1664.75 & & & & H5N3 & 12 & \\
\hline 13 & pig2 kidney-NH2-F7-PGC-F1 & $44.3 \sim 45.3$ & 19.5 & 135254 & 79.0 & 1583.5 & 1598.4 & & & $\mathrm{H} 6 \mathrm{~N} 2$ & 13 & \\
\hline 14 & pig2 kidney-NH2-F7-PGC-F2 & $44.3^{\sim} 45.3$ & 20 & 18303 & 10.6 & 1582.6 & 1598.4 & & & H6N2 & 14 & \\
\hline 15 & pig2 kidney-NH2-F8-PGC-F1 & $45.3 \sim 46.2$ & 18.4 & 20959 & 12.2 & 1624.1 & 1315.3 & & & H5N3 & 15 & \\
\hline 16 & pig2 kidney-NH2-F8-PGC-F2 & $45.3 \sim 46.2$ & 19.1 & 7947 & 4.6 & 1827.7 & & & & H5N4 & 16 & \\
\hline 17 & pig2 kidney-NH2-F8-PGC-F3 & $45.3 \sim 46.2$ & 20.5 & 41298 & 24.1 & 1811.3 & 1686.7 & & & H4N4F1 & 17 & \\
\hline 18 & pig2 kidney-NH2-F8-PGC-F4 & $45.3 \sim 46.2$ & 22.5 & 9439 & 5.5 & 1770.7 & & & & H5N3F1 & 18 & \\
\hline 19 & pig2 kidney-NH2-F9-PGC-F1 & $46.2 \sim 47$ & 19.6 & 64736 & 37.8 & 1827 & & & & $\mathrm{H} 5 \mathrm{~N} 4$ & 19 & \\
\hline 20 & pig2 kidney-NH2-F10-PGC-F1 & $47 \sim 47.8$ & 16.7 & 19691 & 11.5 & 1541.6 & 1744.6 & & & H7N1 & 20 & \\
\hline 21 & pig2 kidney-NH2-F10-PGC-F2 & $47 \sim 47.8$ & 18.3 & 23803 & 13.9 & 2315.3 & 1785.5 & & & H3N8 & 21 & \\
\hline 22 & pig2 kidney-NH2-F10-PGC-F3 & $47^{\sim} 47.8$ & 19 & 60877 & 35.5 & 1744.6 & 1420.8 & & & H7N2 & 22 & \\
\hline 23 & pig2 kidney-NH2-F10-PGC-F4 & $47 \sim 47.8$ & $19+19.2$ & 48126 & 28.1 & 1744.76 & 1420.5 & & & $\mathrm{H} 7 \mathrm{~N} 2$ & 23 & \\
\hline 24 & pig2 kidney-NH2-F10-PGC-F5 & $47 \sim 47.8$ & $19.2+19.5$ & 34245 & 20.0 & 1744.6 & 1785.6 & & & $\mathrm{H} 7 \mathrm{~N} 2$ & 24 & 1 \\
\hline 25 & pig2 kidney-NH2-F10-PGC-F6 & $47 \sim 47.8$ & $19.5+19.6$ & 27220 & 15.9 & 1785.9 & & & & H6N3 & 25 & 1 \\
\hline 26 & pig2 kidney-NH2-F10-PGC-F7 & $47^{\sim} 47.8$ & $19.6+19.8$ & 34087 & 19.9 & 1827.6 & 1785.5 & & & $\mathrm{H} 5 \mathrm{~N} 4$ & 26 & \\
\hline 27 & pig2 kidney-NH2-F10-PGC-F8 & $47 \sim 47.8$ & 20.4 & 142995 & 83.6 & 1972.5 & 1527.5 & & & H5N4F1 & 27 & \\
\hline 28 & pig2 kidney-NH2-F12-PGC-F1 & $49.5 \sim 50.2$ & 19.6 & 19400 & 11.3 & 1988 & & & & H6N4 & 28 & \\
\hline 29 & pig2 kidney-NH2-F12-PGC-F2 & $49.5 \sim 50.2$ & $19.6+19.8$ & 38445 & 22.4 & 1988.9 & 1546.2 & & & $\mathrm{H} 6 \mathrm{~N} 4$ & 29 & \\
\hline 30 & pig2 kidney-NH2-F13-PGC-F1 & $50.2 \sim 50.7$ & 16.5 & 12301 & 7.1 & 1703.4 & & & & H8N1 & 30 & \\
\hline 31 & pig2 kidney-NH2-F13-PGC-F2 & $50.2^{\sim} 50.7$ & 18.8 & 108650 & 63.5 & 1906.6 & 1581.7 & & & $\mathrm{H} 8 \mathrm{~N} 2$ & 31 & \\
\hline 32 & pig2 kidney-NH2-F13-PGC-F3 & $50.2^{\sim} 50.7$ & $18.8+19.1$ & 23943 & 13.9 & 1906.6 & & & & $\mathrm{H} 8 \mathrm{~N} 2$ & 32 & \\
\hline 33 & pig2 kidney-NH2-F13-PGC-F4 & $50.2 \sim 50.7$ & 19.6 & 29554 & 17.2 & 1947.7 & & & & H7N3 & 33 & \\
\hline 34 & pig2 kidney-NH2-F13-PGC-F5 & $50.2 \sim 50.7$ & $20.3+20.4$ & 75436 & 44.1 & 2134.8 & 1687.8 & & & H6N4F1 & 34 & \\
\hline 35 & pig2 kidney-NH2-F15-PGC-F1 & $51.7 \sim 52.1$ & 16.3 & 16879 & 9.8 & 1865.18 & 2079.99 & & & H9N1 & 35 & \\
\hline 36 & pig2 kidney-NH2-F15-PGC-F2 & $51.7 \sim 52.1$ & 19.7 & 12548 & 7.3 & 2338.4 & & & & H6N5F1 & 36 & \\
\hline 37 & pig2 kidney-NH2-F15-PGC-F3 & $51.7 \sim 52.1$ & 20.3 & 12345 & 7.2 & 2337.8 & & & & H6N5F1 & 37 & \\
\hline 38 & pig2 kidney-NH2-F16-PGC-F1 & $52.1 \sim 52.7$ & 18.6 & 39143 & 22.8 & 1947.9 & 1504.9 & & & H7N3 & 38 & \\
\hline 39 & pig2 kidney-NH2-F17-PGC-F1 & $52.7^{\sim} 52.9$ & 19.7 & 141568 & 82.7 & 2151.6 & 1707.1 & & & H7N4 & 39 & \\
\hline 40 & pig2 kidney-NH2-F18-PGC-F1 & $52.9 \sim 53.4$ & 18.7 & 61367 & 35.8 & 2068.7 & & & & H9N2 & 40 & \\
\hline 41 & pig2 kidney-NH2-F18-PGC-F2 & $52.9^{\sim} 53.4$ & $18.7+18.8$ & 42826 & 25.0 & 2069.5 & 2557.5 & & & H9N2 & 41 & \\
\hline 42 & pig2 kidney-NH2-F18-PGC-F3 & $52.9 \sim 53.4$ & 19.7 & 24775 & 14.4 & 2150.1 & 2500.3 & & & H7N4 & 42 & \\
\hline 43 & pig2 kidney-NH2-F18-PGC-F4 & $52.9 \sim 53.4$ & 20.5 & 431348 & 252.2 & 2296.3 & 1849.4 & & & H7N4F1 & 43 & \\
\hline 44 & pig2 kidney-NH2-F19-PGC-F1 & $53.4 \sim 53.9$ & 19.5 & 17548 & 10.2 & 2703.2 & 2500 & & & H7N6F1 & 44 & \\
\hline 45 & pig2 kidney-NH2-F21-PGC-F1 & $55.4 \sim 55.9$ & 19.7 & 35224 & 20.5 & 2442.3 & 2516.2 & & & H7N4F2 & 45 & \\
\hline 46 & pig2 kidney-NH2-F21-PGC-F2 & $55.4 \sim 55.9$ & 20.1 & 10368 & 6.0 & 2443.6 & 2662.4 & & & H7N4F2 & 46 & \\
\hline 47 & pig2 kidney-NH2-F22-PGC-F1 & $55.9 \sim 56.2$ & 19.8 & 12187 & 7.1 & 2662.2 & & & & H8N5F1 & 47 & \\
\hline 48 & pig2 kidney-NH2-F23-PGC-F1 & $56.2 \sim 56.7$ & 19.2 & 19423 & 11.3 & 2474.6 & & & & H9N4 & 48 & \\
\hline 49 & pig2 kidney-NH2-F23-PGC-F2 & $56.2 \sim 56.7$ & $19.5+19.7$ & 19303 & 11.2 & 2662.1 & 2865.3 & & & H8N5F1 & 1 & \\
\hline 50 & pig2 kidney-NH2-F24-PGC-F2 & $56.7 \sim 57.3$ & 19.2 & 44788 & 26.1 & 2678.2 & & & & H9N5 & 2 & \\
\hline 51 & pig2 kidney-NH2-F24-PGC-F1 & $56.7 \sim 57.3$ & 18.5 & 33536 & 19.6 & 2474 & & & & H9N4 & 3 & \\
\hline 52 & pig2 kidney-NH2-F24-PGC-F3 & $56.7 \sim 57.3$ & 19.8 & 19899 & 11.6 & 2679.7 & 2825.6 & & & H9N5 & 4 & \\
\hline 53 & pig2 kidney-NH2-F25-PGC-F1 & $57.3^{\sim} 58.4$ & 19.2 & 32061 & 18.7 & 2883 & 2679 & & & H4N10 & 5 & \\
\hline 54 & pig2 kidney-NH2-F25-PGC-F2 & $57.3^{\sim} 58.4$ & 19.8 & 16337 & 9.5 & 2824 & 3027.2 & & & H9N5F1 & 6 & \\
\hline 55 & pig2 kidney-NH2-F25-PGC-F3 & $57.3 \sim 58.4$ & 20.55 & 19644 & 11.4 & 2823.9 & & & & H9N5F1 & 7 & \\
\hline 56 & pig2 kidney-NH2-F26-PGC-F1 & $58.4 \sim 59.3$ & 19.2 & 46746 & 27.3 & 3045.1 & 2841.2 & & & H5N10 & 8 & \\
\hline 57 & pig2 kidney-NH2-F26-PGC-F2 & $58.4 \sim 59.3$ & 19.9 & 71212 & 41.6 & 3189.7 & & & & H10N6F1 & 9 & \\
\hline 58 & pig2 kidney-NH2-F26-PGC-F3 & $58.4 \sim 59.3$ & $20.6+20.7$ & 17809 & 10.4 & & & 1893.7 & 1731.7 & N.D. & 10 & \\
\hline 59 & pig2 kidney-NH2-F26-PGC-F4 & $58.4 \sim 59.3$ & 21.3 & 13960 & 8.1 & & & 1878.3 & & N.D. & 11 & \\
\hline 60 & pig2 kidney-NH2-F27-PGC-F1 & $59.3 \sim 60.5$ & 19 & 14242 & 8.3 & 3003.3 & & 3138.1 & 2985.4 & N.D. & 12 & \\
\hline 61 & pig2 kidney-NH2-F27-PGC-F2 & $59.3 \sim 60.5$ & $19+19.3$ & 54534 & 31.8 & 3206.3 & 3004.3 & 3341.5 & 3138.2 & N.D. & 13 & \\
\hline 62 & pig2 kidney-NH2-F27-PGC-F3 & $59.3 \sim 60.5$ & 20.8 & 19213 & 11.2 & 1870.7 & 1827.4 & 2097.6 & & N.D. & 14 & \\
\hline 63 & pig2 kidney-NH2-F28-PGC-F1 & $60.5 \sim 62.3$ & 20 & 91188 & 53.3 & 3351.97 & & 3487 & 3334 & N.D. & 15 & \\
\hline 64 & pig2 kidney-NH2-F29-PGC-F1 & $62.3 \sim 63$ & 20.2 & 24014 & 14.0 & & & 2098.1 & 2260 & N.D. & 16 & \\
\hline 65 & pig2 kidney-NH2-F29-PGC-F2 & $62.3 \sim 63$ & $20.5+20.8$ & 81390 & 47.5 & & & 2258.8 & 2081 & N.D. & 17 & \\
\hline 66 & pig2 kidney-NH2-F29-PGC-F3 & $62.3 \sim 63$ & $21.4+21.6$ & 50925 & 29.7 & 2016.4 & 1973.5 & 2243.5 & & N.D. & 18 & \\
\hline 67 & pig2 kidney-NH2-F30-PGC-F1 & $63 \sim 63.8$ & 19 & 43790 & 25.5 & & & 1894.4 & & N.D. & 19 & \\
\hline 68 & pig2 kidney-NH2-F30-PGC-F2 & $63 \sim 63.8$ & $19.65+19.95$ & 21099 & 12.3 & & & 2057.2 & 2464 & N.D. & 20 & \\
\hline 69 & pig2 kidney-NH2-F30-PGC-F3 & $63 \sim 63.8$ & $19.95+20.2$ & 56270 & 32.8 & 1871.5 & 1827.4 & 2098 & & N.D. & 21 & \\
\hline
\end{tabular}




\begin{tabular}{|c|c|c|c|c|c|c|c|c|c|c|c|}
\hline 70 & pig2 kidney-NH2-F30-PGC-F4 & $63 \sim 63.8$ & 20.9 & 197168 & 115.3 & 2017.5 & 1973 & 2494 & 2243.8 & N.D. & 22 \\
\hline 71 & pig2 kidney-NH2-F30-PGC-F5 & $63 \sim 63.8$ & 21.6 & 114011 & 66.6 & & & 2406.8 & 2259.4 & N.D. & 23 \\
\hline 72 & pig2 kidney-NH2-F31-PGC-F1 & $63.8^{\sim} 64.8$ & 20.4 & 9593 & 5.5 & & & 2975.95 & & N.D. & 24 \\
\hline 73 & pig2 kidney-NH2-F31-PGC-F2 & $63.8 \sim 64.8$ & 20.8 & 20902 & 12.2 & 2705.7 & 2747.8 & 2406 & 2259 & N.D. & 25 \\
\hline 74 & pig2 kidney-NH2-F31-PGC-F3 & $63.8 \sim 64.8$ & 21.4 & 37229 & 21.7 & 2182.1 & 2402.9 & 2420.5 & & N.D. & 26 \\
\hline 75 & pig2 kidney-NH2-F32-PGC-F1 & $64.8^{\sim} 66$ & $19.4+19.6$ & 56435 & 32.9 & & & 2056.9 & 2259.9 & N.D. & 27 \\
\hline 76 & pig2 kidney-NH2-F32-PGC-F2 & $64.8^{\sim} 66$ & 20.75 & 34476 & 20.1 & & & 2420.9 & & N.D. & 28 \\
\hline 77 & pig2 kidney-NH2-F32-PGC-F3 & $64.8 \sim 66$ & 21.2 & 19947 & 11.6 & & & 2933.8 & & N.D. & 29 \\
\hline 78 & pig2 kidney-NH2-F33-PGC-F1 & $66^{\sim} 66.9$ & 20.6 & 16862 & 9.8 & 3230.6 & 3189.2 & & & N.D. & 30 \\
\hline 79 & pig2 kidney-NH2-F34-PGC-F1 & $66.9 \sim 67.8$ & 20 & 29491 & 17.2 & 1973 & & 2455 & 2209 & H5N4F1S1 & 31 \\
\hline 80 & pig2 kidney-NH2-F34-PGC-F2 & $66.9 \sim 67.8$ & 20.2 & 16968 & 9.9 & 3191 & 1973 & 3425 & & H10N6F1S1 & 32 \\
\hline 81 & pig2 kidney-NH2-F35-PGC-F1 & $67.8 \sim 69$ & 20.1 & 9045 & 5.2 & & & 2185.9 & & N.D. & 33 \\
\hline 82 & pig2 kidney-NH2-F35-PGC-F2 & $67.8 \sim 69$ & 22.1 & 14970 & 8.7 & & & 2389.7 & & N.D. & 34 \\
\hline 83 & pig2 kidney-NH2-F36-PGC-F1 & $75^{\sim} 76.4$ & 22.7 & 43345 & 25.3 & & & 2536 & & N.D. & 35 \\
\hline 84 & pig2 kidney-NH2-F37-PGC-F1 & $76.4^{\sim 77.9}$ & 21.3 & 15102 & 8.8 & & & 2755.1 & 2389.7 & N.D. & 36 \\
\hline 85 & pig2 kidney-NH2-F37-PGC-F2 & $76.4 \sim 77.9$ & 22 & 52097 & 30.4 & & & 2536.3 & 2902.2 & N.D. & 37 \\
\hline 86 & pig2 kidney-NH2-F37-PGC-F3 & 76.4 77.9 & 22.5 & 15721 & 9.1 & & & 2550 & & N.D. & 38 \\
\hline 87 & pig2 kidney-NH2-F38-PGC-F1 & $77.9 \sim 81$ & 20.6 & 114560 & 66.9 & & & 2389.7 & 2641 & N.D. & 39 \\
\hline 88 & pig2 kidney-NH2-F38-PGC-F2 & $77.9 \sim 81$ & 21.3 & 706513 & 413.2 & 1973 & 2014 & 2535.8 & 2786.9 & H5N4F1S2 & 40 \\
\hline 89 & pig2 kidney-NH2-F38-PGC-F3 & $77.9 \sim 81$ & $21.3+21.8$ & 280971 & 164.3 & 1624 & 1973 & 2209 & 1864.7 & H5N3S2 & 41 \\
\hline 90 & pig2 kidney-NH2-F39-PGC-F1 & $81 \sim 82$ & 21 & 58530 & 34.2 & & & 2551.4 & & N.D. & 42 \\
\hline 91 & pig2 kidney-NH2-F40-PGC-F1 & $82 \sim 83.2$ & 21.3 & 26169 & 15.2 & & & 2550 & & N.D. & 43 \\
\hline 92 & pig2 kidney-NH2-F41-PGC-F1 & $83.2 \sim 84.8$ & $20.8+21$ & 6291 & 3.6 & & & 2574 & & N.D. & 44 \\
\hline 93 & pig2 kidney-NH2-F41-PGC-F2 & $83.2 \sim 84.8$ & $21+21.1$ & 13454 & 7.8 & & & 2575.8 & 2264.8 & N.D. & 45 \\
\hline 94 & pig2 kidney-NH2-F44-PGC-F1 & $95.2 \sim 97.6$ & 22.8 & 12659 & 7.3 & & & 3200.9 & 2907.5 & N.D. & 46 \\
\hline 95 & pig2 kidney-NH2-F45-PGC-F1 & $97.6^{\sim 99.2}$ & 21.9 & 23845 & 13.9 & & & 3200.9 & 2908 & N.D. & 47 \\
\hline 96 & pig2 kidney-NH2-F46-PGC-F1 & $99.2 \sim 100.2$ & 22.9 & 35498 & 20.7 & & & 3204.2 & 2910 & N.D. & 48 \\
\hline
\end{tabular}


Table S7. NGGM raw data of lectin-bindings with pig kidney shotgun array. Column A shows the number of fractions of combined library; Column B to $J$ shows the related copy number after normalizing the total number of one sample to 1 million.

\begin{tabular}{|c|c|c|c|c|c|c|c|c|c|}
\hline & \multicolumn{9}{|c|}{ Fold of negative control (Copy number compared to Blank) } \\
\hline $\begin{array}{l}\text { Fraction } \\
\text { No. }\end{array}$ & $\begin{array}{l}\text { ConA 100 } \\
\text { ug/ml, } \\
1.25 f m o l ~ p i g \\
\text { kidney } \\
\text { library }\end{array}$ & \begin{tabular}{|l|} 
AAL 100 \\
ug/ml, $1.25 \mathrm{fmol}$ \\
pig kidney \\
library
\end{tabular} & \begin{tabular}{|l|} 
RCA-I 100 \\
ug/ml, \\
$1.25 f m o l ~ p i g$ \\
kidney \\
library
\end{tabular} & 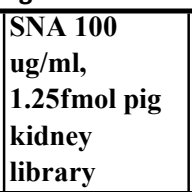 & 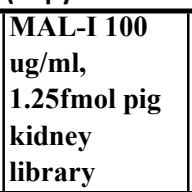 & \begin{tabular}{|l|} 
HPA 100 \\
ug/ml, \\
1.25 fmol pig \\
kidney \\
library
\end{tabular} & \begin{tabular}{|l|} 
PNA 100 \\
ug/ml, \\
$1.25 f m o l ~ p i g$ \\
kidney \\
library
\end{tabular} & 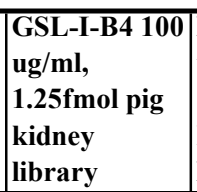 & 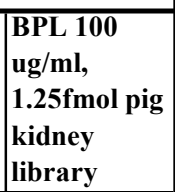 \\
\hline 1 & 309 & 400 & 1235 & 19541 & 16497 & 21255 & 78470 & 638 & 6765 \\
\hline 2 & 74 & 129 & 517 & 6476 & 654 & 5031 & 2380 & 215 & 1919 \\
\hline 3 & 80 & 124 & 135 & 2094 & 993 & 1982 & 1379 & 78 & 827 \\
\hline 4 & 2267 & 484 & 1028 & 30521 & 8377 & 18630 & 7026 & 504 & 5686 \\
\hline 5 & 558 & 172 & 809 & 6297 & 2000 & 4379 & 1984 & 153 & 1316 \\
\hline 6 & 3046 & 361 & 1112 & 6557 & 5561 & 89027 & 60715 & 323 & 3948 \\
\hline 7 & 19212 & 214 & 567 & 6761 & 2447 & 7127 & 10973 & 373 & 4765 \\
\hline 8 & 1438 & 291 & 704 & 16152 & 2449 & 16915 & 5556 & 414 & 4714 \\
\hline 9 & 1755 & 609 & 644 & 8507 & 4980 & 9539 & 28107 & 248 & 2622 \\
\hline 10 & 9995 & 154 & 406 & 5840 & 1148 & 5806 & 6617 & 219 & 2711 \\
\hline 11 & 919 & 260 & 750 & 4750 & 1891 & 6019 & 8168 & 334 & 3690 \\
\hline 12 & 3328 & 262 & 1352 & 4371 & 2855 & 7779 & 9903 & 497 & 6287 \\
\hline 13 & 118237 & 368 & 1016 & 3360 & 1924 & 4207 & 9263 & 505 & 3647 \\
\hline 14 & 5837 & 886 & 902 & 13797 & 4966 & 5086 & 7304 & 295 & 6108 \\
\hline 15 & 1549 & 584 & 2189 & 7997 & 2287 & 5192 & 8147 & 322 & 2912 \\
\hline 16 & 3595 & 463 & 1312 & 12863 & 1873 & 14145 & 6313 & 329 & 4310 \\
\hline 17 & 3742 & 1908 & 1671 & 5269 & 1586 & 5762 & 6844 & 365 & 3966 \\
\hline 18 & 637 & 701 & 1341 & 6621 & 2037 & 7917 & 5940 & 297 & 4220 \\
\hline 19 & 498 & 111 & 1025 & 1352 & 813 & 1393 & \begin{tabular}{|l|}
1077 \\
\end{tabular} & 70 & 966 \\
\hline 20 & 6493 & 204 & 726 & 11272 & 1724 & 7540 & 7452 & 674 & 5200 \\
\hline 21 & 667 & 512 & 4604 & 21355 & 2150 & 22202 & 7004 & 735 & 6945 \\
\hline 22 & 80916 & 783 & 4597 & 12716 & 4538 & 13225 & 11837 & 974 & 9880 \\
\hline 23 & 50458 & 341 & 1427 & 3553 & 2191 & 3426 & 5029 & 442 & 2468 \\
\hline 24 & 81025 & 1574 & 4047 & 18292 & 2865 & 19155 & 10198 & 1223 & 8776 \\
\hline 25 & 17422 & 1067 & 5309 & 4433 & 1941 & 7640 & 6479 & 4630 & 5691 \\
\hline 26 & 1450 & 1175 & 1081 & 2612 & 2204 & 6659 & 6422 & 585 & 4596 \\
\hline 27 & 2366 & 5796 & 19263 & 13368 & 4678 & 14126 & 11537 & 971 & 7649 \\
\hline 28 & 2677 & 1281 & 2873 & 18536 & 2272 & 15340 & 9900 & 720 & 7499 \\
\hline 29 & 1374 & 1594 & 3672 & 3608 & 1812 & 5140 & 8644 & 788 & 5059 \\
\hline 30 & 14835 & 539 & 1588 & 15974 & 1988 & 18485 & 7891 & 686 & 10038 \\
\hline 31 & 190636 & 954 & 8438 & 7027 & 2906 & 5797 & 13548 & 1265 & 6368 \\
\hline 32 & 18424 & 1134 & 4954 & 13255 & 4554 & 11491 & 9515 & 1228 & 9518 \\
\hline 33 & 2340 & 17049 & 23287 & 18404 & 2359 & 15917 & 9071 & 1618 & 33293 \\
\hline 34 & 1910 & 4731 & 16969 & 7431 & 4935 & 7548 & 13257 & 2048 & 10626 \\
\hline 35 & 93089 & 553 & 3147 & 10042 & 2622 & 6748 & 11722 & 1118 & 7678 \\
\hline 36 & 516 & 5445 & 12665 & 6862 & 3130 & 2877 & 9640 & 2244 & 6499 \\
\hline 37 & 1346 & 2214 & 35334 & 3444 & 2380 & 3210 & 7206 & 1246 & 6765 \\
\hline 38 & 2659 & 479 & 2788 & 2164 & 2711 & 4042 & 7671 & 9247 & 5097 \\
\hline 39 & 1374 & 11307 & 7314 & 4813 & 1972 & 5207 & 6538 & 36765 & 8309 \\
\hline 40 & 60163 & 1009 & 17495 & 9554 & 3122 & 6831 & 7639 & 7380 & 7049 \\
\hline 41 & 7067 & 1211 & 40944 & 18201 & 3583 & 13543 & 7240 & 7665 & 7392 \\
\hline 42 & 761 & 8579 & 21771 & 54009 & 2896 & 19701 & 9549 & 3928 & 10383 \\
\hline 43 & 909 & 5364 & 4825 & 5404 & 2413 & 6221 & 6087 & 45720 & 7626 \\
\hline 44 & 326 & 8769 & 112990 & 7469 & 3694 & 8734 & 6507 & 6496 & 9120 \\
\hline 45 & 437 & 71863 & 13004 & 5390 & 2471 & 7060 & 11176 & 33942 & 7633 \\
\hline 46 & 523 & 43268 & 9188 & 3500 & 2158 & 6942 & 8920 & 23468 & 7457 \\
\hline 47 & 268 & 13941 & 8810 & 2933 & 1493 & 4991 & 6280 & 12567 & 6004 \\
\hline 48 & 502 & 14769 & 19266 & 9058 & 2031 & 11395 & 9301 & 44760 & 17274 \\
\hline 49 & 1238 & 5817 & 11013 & 3292 & 2542 & 4588 & 4796 & 3065 & 5633 \\
\hline 50 & 8128 & 453184 & 36095 & 9100 & 20714 & 7202 & 35117 & 184363 & 60553 \\
\hline 51 & 1130 & 1072 & 2257 & 2469 & 1629 & 6528 & 7330 & 5495 & 8800 \\
\hline 52 & 1211 & 5143 & 9163 & 2638 & 3159 & 3804 & 5767 & 6341 & 8859 \\
\hline 53 & 2194 & 26666 & 9982 & 6272 & 2883 & 5029 & 10417 & 18485 & 18965 \\
\hline 54 & 1071 & 3452 & 6181 & 5410 & 10481 & 5171 & 16469 & 35767 & 70225 \\
\hline
\end{tabular}




\begin{tabular}{|c|c|c|c|c|c|c|c|c|c|}
\hline 55 & 1696 & 3647 & 7469 & 3262 & 2033 & 9998 & 7319 & 31327 & 12667 \\
\hline 56 & 10164 & 17256 & 18297 & 6067 & 3264 & 25074 & 11190 & 26562 & 18933 \\
\hline 57 & 4941 & 9979 & 19499 & 13048 & 6267 & 9342 & 8564 & 27252 & 18331 \\
\hline 58 & 1016 & 763 & 1246 & 2408 & 2305 & 2137 & 2619 & 1092 & 2760 \\
\hline 59 & 4123 & 2796 & 3658 & 9657 & 2220 & 6379 & 9814 & 3857 & 14099 \\
\hline 60 & 4009 & 13288 & 3881 & 7958 & 3142 & 21454 & 8829 & 35526 & 18066 \\
\hline 61 & 2727 & 4725 & 3005 & 1370 & 3254 & 6585 & 6831 & 22300 & 10622 \\
\hline 62 & 3293 & 2114 & 1970 & 2582 & 8484 & 5090 & 6378 & 3352 & 7395 \\
\hline 63 & 3130 & 4422 & 13739 & 3615 & 2825 & 9397 & 7227 & 114809 & 41241 \\
\hline 64 & 3579 & 5991 & 17236 & 5300 & 4161 & 9355 & 7830 & 18679 & 13441 \\
\hline 65 & 12487 & 7299 & 29689 & 4670 & 4637 & 7882 & 10367 & 11862 & 11997 \\
\hline 66 & 6597 & 5003 & 4681 & 2350 & 9172 & 6326 & 7808 & 1712 & 5053 \\
\hline 67 & 9875 & 16165 & 11943 & 12003 & 2600 & 56774 & 17953 & 18093 & 10957 \\
\hline 68 & 8837 & 8686 & 25816 & 5237 & 7975 & 17559 & 15475 & 29561 & 20259 \\
\hline 69 & 8826 & 8258 & 30874 & 5689 & 4563 & 11635 & 10886 & 13216 & 13891 \\
\hline 70 & 12995 & 6347 & 27531 & 6495 & 8642 & 6065 & 13223 & 7176 & 9190 \\
\hline 71 & 8923 & 8914 & 9255 & 2005 & 7970 & 7806 & 10220 & 4189 & 7571 \\
\hline 72 & 1531 & 3435 & 8082 & 4314 & 6377 & 5985 & 8246 & 3928 & 6635 \\
\hline 73 & 3136 & 3708 & 19681 & 5771 & 3766 & 5335 & 7838 & 5748 & 13143 \\
\hline 74 & 4308 & 26417 & 14038 & 4778 & 8946 & 9662 & 11100 & 6433 & 13008 \\
\hline 75 & 7053 & 11377 & 13320 & 8033 & 6139 & 21526 & 14375 & 18016 & 13495 \\
\hline 76 & 5765 & 9044 & 16975 & 4853 & 8637 & 11096 & 11633 & 13580 & 14520 \\
\hline 77 & 2102 & 8426 & 5315 & 2104 & 3832 & 6457 & 6538 & 5014 & 9225 \\
\hline 78 & 2370 & 5078 & 7935 & 4341 & 6374 & 8792 & 9958 & 6747 & 9117 \\
\hline 79 & 5932 & 24606 & 104391 & 27859 & 10270 & 37437 & 18756 & 18140 & 41942 \\
\hline 80 & 5258 & 7742 & 7809 & 7356 & 10276 & 20989 & 17591 & 10219 & 8583 \\
\hline 81 & 1078 & 1583 & 1174 & 8903 & 23508 & 6271 & 6166 & 1131 & 5295 \\
\hline 82 & 696 & 1148 & 2784 & 6263 & 34348 & 4889 & 6210 & 2214 & 9832 \\
\hline 83 & 2051 & 4916 & 4996 & 11172 & 162860 & 6254 & 9200 & 6495 & 8075 \\
\hline 84 & 1305 & 2251 & 4183 & 7688 & 46941 & 6111 & 6877 & 2462 & 4609 \\
\hline 85 & 965 & 3257 & 3489 & 6401 & 52031 & 4648 & 7675 & 3206 & 5964 \\
\hline 86 & 1124 & 1898 & 2627 & 6004 & 40981 & 5013 & 7992 & 1507 & 7351 \\
\hline 87 & 1555 & 2385 & 5718 & 39180 & 11728 & 6158 & 7610 & 1202 & 10800 \\
\hline 88 & 1072 & 2526 & 6326 & 37608 & 5845 & 5025 & 6365 & 1118 & 5929 \\
\hline 89 & 1890 & 3915 & 8411 & 29885 & 20778 & 5986 & 8342 & 1449 & 10315 \\
\hline 90 & 1032 & 2396 & 6549 & 27722 & 13431 & 4653 & 6746 & 1025 & 9044 \\
\hline 91 & 969 & 1766 & 2827 & 14447 & 15737 & 5140 & 8013 & 721 & 6889 \\
\hline 92 & 2421 & 2393 & 1948 & 12199 & 11271 & 12519 & 9901 & 1237 & 5842 \\
\hline 93 & 1336 & 1961 & 6660 & 10870 & 16314 & 8931 & 8814 & 1025 & 16670 \\
\hline 94 & 829 & 913 & 2497 & 4487 & 135141 & 3841 & 5690 & 510 & 7960 \\
\hline 95 & 721 & 1120 & 2208 & 15919 & 42216 & 4707 & 6352 & 617 & 6317 \\
\hline 96 & 1065 & 1111 & 3482 & 82737 & 28720 & 4998 & 7505 & 400 & 11326 \\
\hline 2-FL & 184 & 7563 & 771 & 1368 & 1937 & 4290 & 5215 & 383 & 5876 \\
\hline NC & 54 & 95 & 235 & 1041 & 1545 & 3723 & 7443 & 80 & 3462 \\
\hline
\end{tabular}


Table S7. NGGM raw data of lectin-bindings with pig kidney shotgun array. Column A shows the number of fractions of sub-library 1 ;

Column B to $L$ shows the exact copy number from $1 \mathrm{M}$-reads NGS sequencing.

\begin{tabular}{|c|c|c|c|c|c|c|c|c|c|c|c|}
\hline $\begin{array}{l}\text { Fraction } \\
\text { No. }\end{array}$ & $\begin{array}{l}\text { blank, } \\
1.25 \text { fmol } \\
\text { pig } \\
\text { kidney } \\
\text { library } 1\end{array}$ & $\begin{array}{l}\text { ConA 100 } \\
\text { ug/ml, } \\
1.25 f m o l \\
\text { pig } \\
\text { kidney } \\
\text { library } 1\end{array}$ & $\begin{array}{l}\text { AAL } 100 \\
\text { ug/ml, } \\
1.25 \text { fmol } \\
\text { pig } \\
\text { kidney } \\
\text { library } 1\end{array}$ & \begin{tabular}{|l|} 
RCA-I \\
100 \\
ug/ml, \\
$1.25 f m o l$ \\
pig \\
kidney \\
library 1 \\
\end{tabular} & $\begin{array}{l}\text { SNA } 100 \\
\text { ug/ml, } \\
1.25 f m o l \\
\text { pig } \\
\text { kidney } \\
\text { library } 1\end{array}$ & \begin{tabular}{|l} 
MAL-I \\
100 \\
ug/ml, \\
$1.25 f m o l$ \\
pig \\
kidney \\
library 1
\end{tabular} & $\begin{array}{l}\text { HPA } 100 \\
\text { ug/ml, } \\
1.25 f m o l \\
\text { pig } \\
\text { kidney } \\
\text { library } 1\end{array}$ & $\begin{array}{l}\text { PNA 100 } \\
\text { ug/ml, } \\
1.25 f \text { mol } \\
\text { pig } \\
\text { kidney } \\
\text { library } 1\end{array}$ & \begin{tabular}{|l|} 
GSL-I-B4 \\
100 \\
ug/ml, \\
$1.25 f m o l$ \\
pig \\
kidney \\
library 1 \\
\end{tabular} & \begin{tabular}{|l} 
BPL 100 \\
ug/ml, \\
$1.25 \mathrm{fmol}$ \\
pig \\
kidney \\
library 1
\end{tabular} & $\begin{array}{l}\text { input, pig } \\
\text { kidney } \\
\text { library } 1\end{array}$ \\
\hline 1 & 1374 & 100 & 371 & 443 & 3613 & 7992 & 3018 & 11386 & 415 & 831 & 540 \\
\hline 2 & 8459 & 147 & 735 & 1142 & 7372 & 1952 & 4398 & 2126 & 862 & 1451 & 515 \\
\hline 3 & 2988 & 56 & 250 & 105 & 842 & 1046 & 612 & 435 & 110 & 221 & 641 \\
\hline 4 & 775 & 414 & 253 & 208 & 3183 & 2289 & 1492 & 575 & 185 & 394 & 577 \\
\hline 5 & 4004 & 526 & 465 & 846 & 3393 & 2823 & 1812 & 839 & 290 & 471 & 681 \\
\hline 6 & 255 & 183 & 62 & 74 & 225 & 500 & 2346 & 1635 & 39 & 90 & 607 \\
\hline 7 & 277 & 1254 & 40 & 41 & 252 & 239 & 204 & 321 & 49 & 118 & 538 \\
\hline 8 & 484 & 164 & 95 & 89 & 1052 & 418 & 846 & 284 & 95 & 204 & 615 \\
\hline 9 & 1540 & 637 & 632 & 259 & 1763 & 2704 & 1518 & 4571 & 181 & 361 & 729 \\
\hline 10 & 425 & 1001 & 44 & 45 & 334 & 172 & 255 & 297 & 44 & 103 & 540 \\
\hline 11 & 291 & 63 & 51 & 57 & 186 & 194 & 181 & 251 & 46 & 96 & 568 \\
\hline 12 & 153 & 120 & 27 & 54 & 90 & 154 & 123 & 160 & 36 & 86 & 536 \\
\hline 13 & 230 & 6408 & 57 & 61 & 104 & 156 & 100 & 225 & 55 & 75 & 594 \\
\hline 14 & 293 & 403 & 175 & 69 & 544 & 513 & 154 & 226 & 41 & 160 & 557 \\
\hline 15 & 315 & 115 & 124 & 180 & 339 & 254 & 169 & 271 & 48 & 82 & 419 \\
\hline 16 & 327 & 277 & 102 & 112 & 566 & 216 & 478 & 218 & 51 & 126 & 477 \\
\hline 17 & 220 & 194 & 283 & 96 & 156 & 123 & 131 & 159 & 38 & 78 & 624 \\
\hline 18 & 220 & 33 & 104 & 77 & 196 & 158 & 180 & 138 & 31 & 83 & 534 \\
\hline 19 & 1424 & 167 & 107 & 381 & 259 & 408 & 205 & 162 & 47 & 123 & 435 \\
\hline 20 & 385 & 589 & 53 & 73 & 584 & 234 & 300 & 303 & 123 & 179 & 603 \\
\hline 21 & 649 & 102 & 224 & 780 & 1865 & 492 & 1489 & 480 & 226 & 403 & 460 \\
\hline 22 & 180 & 3432 & 95 & 216 & 308 & 288 & 246 & 225 & 83 & 159 & 553 \\
\hline 23 & 435 & 5172 & 100 & 162 & 208 & 336 & 154 & 231 & 91 & 96 & 535 \\
\hline 24 & 195 & 3723 & 207 & 206 & 480 & 197 & 386 & 210 & 113 & 153 & 496 \\
\hline 25 & 57 & 234 & 41 & 79 & 34 & 39 & 45 & 39 & 125 & 29 & 117 \\
\hline 26 & 202 & 69 & 160 & 57 & 71 & 157 & 139 & 137 & 56 & 83 & 446 \\
\hline 27 & 174 & 97 & 680 & 875 & 313 & 287 & 254 & 212 & 80 & 119 & 450 \\
\hline 28 & 176 & 111 & 152 & 132 & 439 & 141 & 279 & 184 & 60 & 118 & 374 \\
\hline 29 & 241 & 78 & 259 & 231 & 117 & 154 & 128 & 220 & 90 & 109 & 514 \\
\hline 30 & 234 & 818 & 85 & 97 & 503 & 164 & 447 & 195 & 76 & 210 & 578 \\
\hline 31 & 202 & 9074 & 130 & 445 & 191 & 207 & 121 & 289 & 121 & 115 & 493 \\
\hline 32 & 208 & 903 & 159 & 269 & 371 & 334 & 247 & 209 & 121 & 177 & 546 \\
\hline 33 & 214 & 118 & 2460 & 1301 & 530 & 178 & 352 & 205 & 164 & 637 & 466 \\
\hline 34 & 100 & 45 & 319 & 443 & 100 & 174 & 78 & 140 & 97 & 95 & 467 \\
\hline 35 & 185 & 4058 & 69 & 152 & 250 & 171 & 129 & 229 & 98 & 127 & 582 \\
\hline 36 & 222 & 27 & 815 & 734 & 205 & 245 & 66 & 226 & 236 & 129 & 497 \\
\hline 37 & 205 & 65 & 306 & 1891 & 95 & 172 & 68 & 156 & 121 & 124 & 573 \\
\hline 38 & 158 & 99 & 51 & 115 & 46 & 151 & 66 & 128 & 692 & 72 & 491 \\
\hline 39 & 210 & 68 & 1601 & |401 & 136 & 146 & 113 & 145 & 3657 & 156 & 618 \\
\hline 40 & 238 & 3374 & 162 & 1087 & 306 & 262 & 168 & 192 & 832 & 150 & 465 \\
\hline 41 & 463 & 771 & 378 & 4949 & 1134 & 585 & 648 & 354 & 1681 & 306 & 609 \\
\hline 42 & \begin{tabular}{|l|}
474 \\
\end{tabular} & 85 & 2742 & 2694 & 3445 & 484 & 965 & 478 & 882 & 440 & 580 \\
\hline 43 & 154 & 33 & 557 & 194 & 112 & 131 & 99 & 99 & 3335 & 105 & 536 \\
\hline 44 & 195 & 15 & 1153 & 5752 & 196 & 254 & 176 & 134 & 600 & 159 & 598 \\
\hline 45 & 233 & 24 & 11290 & 791 & 169 & 203 & 170 & 275 & 3746 & 159 & 609 \\
\hline 46 & 138 & 17 & 4026 & 331 & 65 & 105 & 99 & 130 & 1534 & 92 & 447 \\
\hline 47 & 190 & 12 & 1786 & 437 & 75 & 100 & 98 & 126 & 1131 & 102 & 513 \\
\hline 48 & 169 & 20 & 1683 & \begin{tabular}{l|l|}
850 & \\
\end{tabular} & 206 & 121 & 199 & 166 & 3583 & 261 & 535 \\
\hline
\end{tabular}




\begin{tabular}{|l|r|r|r|r|r|r|r|r|r|r|}
\hline $2-\mathrm{FL}$ & 159 & 8 & 816 & 28 & 25 & 98 & 63 & 81 & 29 & 65 \\
\hline $\mathrm{NC}$ & 162 & 1 & 5 & 14 & 27 & 99 & 70 & 134 & 667 \\
\hline
\end{tabular}


Table S7. NGGM raw data of lectin-bindings with pig kidney shotgun array. Column A shows the number of fractions of sub-library 2; Column B to $L$ shows the exact copy number from 1M-reads NGS sequencing.

\begin{tabular}{|c|c|c|c|c|c|c|c|c|c|c|c|}
\hline $\begin{array}{l}\text { Fraction } \\
\text { No. }\end{array}$ & \begin{tabular}{|l|} 
blank, \\
$1.25 f m o l$ \\
pig \\
kidney \\
library 2
\end{tabular} & $\begin{array}{l}\text { ConA 100 } \\
\text { ug/ml, } \\
1.25 f m o l \\
\text { pig } \\
\text { kidney } \\
\text { library } 2\end{array}$ & $\begin{array}{l}\text { AAL } 100 \\
\text { ug/ml, } \\
1.25 \text { fmol } \\
\text { pig } \\
\text { kidney } \\
\text { library } 2\end{array}$ & \begin{tabular}{|l} 
RCA-I \\
100 \\
ug/ml, \\
$1.25 f m o l$ \\
pig \\
kidney \\
library 2
\end{tabular} & $\begin{array}{l}\text { SNA } 100 \\
\text { ug/ml, } \\
1.25 \text { fmol } \\
\text { pig } \\
\text { kidney } \\
\text { library } 2\end{array}$ & \begin{tabular}{|l|} 
MAL-I \\
100 \\
ug/ml, \\
$1.25 f m o l$ \\
pig \\
kidney \\
library 2
\end{tabular} & $\begin{array}{l}\text { HPA } 100 \\
\text { ug/ml, } \\
1.25 f m o l \\
\text { pig } \\
\text { kidney } \\
\text { library } 2\end{array}$ & $\begin{array}{l}\text { PNA } 100 \\
\text { ug/ml, } \\
1.25 \text { fmol } \\
\text { pig } \\
\text { kidney } \\
\text { library } 2\end{array}$ & \begin{tabular}{|l|} 
GSL-I-B4 \\
100 \\
ug/ml, \\
$1.25 f m o l$ \\
pig \\
kidney \\
library 2 \\
\end{tabular} & \begin{tabular}{|l|} 
BPL 100 \\
ug/ml, \\
$1.25 f m o l$ \\
pig \\
kidney \\
library 2
\end{tabular} & $\begin{array}{l}\text { input, pig } \\
\text { kidney } \\
\text { library } 2\end{array}$ \\
\hline 1 & 288 & 180 & 389 & 573 & 178 & 81 & 172 & 186 & 273 & 144 & 559 \\
\hline 2 & 48 & 197 & 5051 & 313 & 82 & 110 & 45 & 227 & 2737 & 258 & 658 \\
\hline 3 & 233 & 133 & 58 & 95 & 108 & 42 & 198 & 230 & 396 & 182 & 744 \\
\hline 4 & 206 & 126 & 246 & 341 & 102 & 72 & 102 & 160 & 404 & 162 & 728 \\
\hline 5 & \begin{tabular}{|l|l|}
417 \\
\end{tabular} & 462 & 2582 & 752 & 491 & 133 & 273 & 585 & 2384 & 702 & 699 \\
\hline 6 & 257 & 139 & 206 & 287 & 261 & 298 & 173 & 570 & 2843 & 1602 & 715 \\
\hline 7 & 209 & 179 & 177 & 282 & 128 & 47 & 272 & 206 & 2025 & 235 & 740 \\
\hline 8 & 144 & 739 & 577 & 476 & 164 & 52 & 470 & 217 & 1183 & 242 & 655 \\
\hline 9 & 287 & 716 & 665 & 1011 & 703 & 199 & 349 & 331 & 2419 & 467 & 975 \\
\hline 10 & 604 & 310 & 107 & 136 & 273 & 154 & 168 & 213 & 204 & 148 & 779 \\
\hline 11 & 171 & 356 & 111 & 113 & 310 & 42 & 142 & 226 & 204 & 214 & 788 \\
\hline 12 & 164 & 332 & 506 & 115 & 245 & 57 & 458 & 195 & 1802 & 263 & 813 \\
\hline 13 & 175 & 241 & 192 & 95 & 45 & 63 & 150 & 161 & 1207 & 165 & 633 \\
\hline 14 & 163 & 271 & 80 & 58 & 79 & 153 & 108 & 140 & 169 & 107 & 826 \\
\hline 15 & 112 & 177 & 115 & 278 & 76 & 35 & 137 & 109 & 3977 & 410 & 707 \\
\hline 16 & 202 & 365 & 281 & 629 & 201 & 93 & 246 & 213 & 1167 & 241 & 772 \\
\hline 17 & 154 & 971 & 261 & 826 & 135 & 79 & 158 & 215 & 565 & 164 & 855 \\
\hline 18 & 136 & 453 & 158 & 115 & 60 & 138 & 112 & 143 & 72 & 61 & 849 \\
\hline 19 & 146 & 728 & 548 & 315 & 329 & 42 & 1079 & 353 & 817 & 142 & 762 \\
\hline 20 & 119 & 531 & 240 & 555 & 117 & 105 & 272 & 248 & 1088 & 214 & 668 \\
\hline 21 & 206 & 918 & 395 & 1149 & 220 & 104 & 312 & 302 & 842 & 254 & 893 \\
\hline 22 & 114 & 748 & 168 & 567 & 139 & 109 & 90 & 203 & 253 & 93 & 1041 \\
\hline 23 & 186 & 838 & 385 & 311 & 70 & 164 & 189 & 256 & 241 & 125 & 919 \\
\hline 24 & 163 & 126 & 130 & 238 & 132 & 115 & 127 & 181 & 198 & 96 & 679 \\
\hline 25 & 36 & 57 & 31 & 128 & 39 & 15 & 25 & 38 & 64 & 42 & 273 \\
\hline 26 & 97 & 211 & 595 & 246 & 87 & 96 & 122 & 145 & 193 & 112 & 729 \\
\hline 27 & 187 & 666 & 494 & 450 & 282 & 127 & 524 & 362 & 1042 & 224 & 734 \\
\hline 28 & 90 & 262 & 189 & 276 & 82 & 86 & 130 & 141 & 378 & 116 & 647 \\
\hline 29 & 276 & 293 & 540 & 265 & 109 & 117 & 232 & 243 & 428 & 226 & 937 \\
\hline 30 & 173 & 207 & 204 & 248 & 141 & 122 & 198 & 232 & 361 & 140 & 852 \\
\hline 31 & 213 & 638 & 1217 & 4017 & 1114 & 242 & 1038 & 538 & 1195 & 793 & 740 \\
\hline 32 & 168 & 446 & 302 & 237 & 232 & 191 & 459 & 398 & 531 & 128 & 739 \\
\hline 33 & 283 & 154 & 104 & 60 & 473 & 736 & 231 & 235 & 99 & 133 & 999 \\
\hline 34 & 165 & 58 & 44 & 83 & 194 & \begin{tabular}{ll|}
627 \\
\end{tabular} & 105 & 138 & 113 & 144 & 854 \\
\hline 35 & 113 & 117 & 129 & 102 & 237 & 2036 & 92 & 140 & 227 & 81 & 910 \\
\hline 36 & 176 & 116 & 92 & 133 & 254 & 914 & 140 & 163 & 134 & 72 & 710 \\
\hline 37 & 119 & 58 & 90 & 75 & 143 & 685 & 72 & 123 & 118 & 63 & 666 \\
\hline 38 & 118 & 67 & 52 & 56 & 133 & 535 & 77 & 127 & 55 & 77 & 685 \\
\hline 39 & 121 & 95 & 67 & 125 & 890 & 157 & 97 & 124 & 45 & 116 & 725 \\
\hline 40 & 133 & 72 & 78 & 152 & 939 & 86 & 87 & 114 & 46 & 70 & 757 \\
\hline 41 & 154 & 147 & 140 & 234 & 864 & 354 & 120 & 173 & 69 & 141 & 804 \\
\hline 42 & 142 & 74 & 79 & 168 & 739 & 211 & 86 & 129 & 45 & 114 & 781 \\
\hline 43 & 139 & 68 & 57 & 71 & 377 & 242 & 93 & 150 & 31 & 85 & 839 \\
\hline 44 & 162 & 198 & 90 & 57 & 371 & 202 & 264 & 216 & 62 & 84 & 926 \\
\hline 45 & 123 & 83 & 56 & 148 & 251 & 222 & 143 & 146 & 39 & 182 & 808 \\
\hline 46 & 184 & 77 & 39 & 83 & 155 & 2751 & 92 & 141 & 29 & 130 & 801 \\
\hline 47 & 173 & 63 & 45 & 69 & 517 & 808 & 106 & 148 & 33 & 97 & 820 \\
\hline 48 & 186 & 100 & 48 & 117 & 2889 & 591 & 121 & 188 & 23 & 187 & 1021 \\
\hline
\end{tabular}




\begin{tabular}{|l|r|r|r|r|r|r|r|r|r|r|}
\hline $2-\mathrm{FL}$ & 102 & 8 & 178 & 16 & 30 & 24 & 63 & 77 & 12 & 65 \\
\hline $\mathrm{NC}$ & 120 & 5 & 4 & 3 & 19 & 18 & 51 & 114 & 345 \\
\hline
\end{tabular}


Table S7. NGGM raw data of lectin-bindings with pig kidney shotgun array. Column A shows the number of fractions of combined library; Column B to J shows the related copy number compared to Blank.

\begin{tabular}{|c|c|c|c|c|c|c|c|c|c|}
\hline & & & Fold of $n$ & negative control & (Copy number & compared to $\mathrm{B}$ & 3lank) & & \\
\hline $\begin{array}{l}\text { Fraction } \\
\text { No. }\end{array}$ & $\begin{array}{l}\text { ConA } 100 \\
\text { ug/ml, } \\
1.25 f m o l ~ p i g \\
\text { kidney } \\
\text { library }\end{array}$ & $\begin{array}{l}\text { AAL } 100 \\
\text { ug/ml, 1.25fmol } \\
\text { pig kidney } \\
\text { library }\end{array}$ & $\begin{array}{l}\text { RCA-I 100 } \\
\text { ug/ml, } \\
1.25 f m o l ~ p i g \\
\text { kidney } \\
\text { library }\end{array}$ & $\begin{array}{l}\text { SNA 100 } \\
\text { ug/ml, } \\
1.25 f m o l ~ p i g \\
\text { kidney } \\
\text { library }\end{array}$ & \begin{tabular}{|l} 
MAL-I 100 \\
ug/ml, \\
$1.25 f m o l ~ p i g$ \\
kidney \\
library
\end{tabular} & 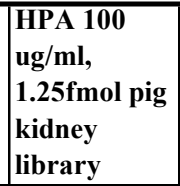 & \begin{tabular}{|l|} 
PNA 100 \\
ug/ml, \\
1.25 fmol pig \\
kidney \\
library
\end{tabular} & 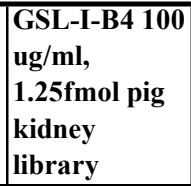 & \begin{tabular}{|l} 
BPL 100 \\
ug/ml, \\
1.25 fmol pig \\
kidney \\
library
\end{tabular} \\
\hline 1 & 7 & 27 & 32 & 263 & 582 & 220 & 829 & 30 & 60 \\
\hline 2 & 2 & 9 & 14 & 87 & 23 & 52 & 25 & 10 & 17 \\
\hline 3 & 2 & 8 & 4 & 28 & 35 & 20 & 15 & 4 & 7 \\
\hline 4 & 53 & 33 & 27 & 411 & 295 & 193 & 74 & 24 & 51 \\
\hline 5 & 13 & 12 & 21 & 85 & 71 & 45 & 21 & 7 & 12 \\
\hline 6 & 72 & 24 & 29 & 88 & 196 & 920 & 641 & 15 & 35 \\
\hline 7 & 453 & 14 & 15 & 91 & 86 & 74 & 116 & 18 & 43 \\
\hline 8 & 34 & 20 & 18 & 217 & 86 & 175 & 59 & 20 & 42 \\
\hline 9 & 41 & 41 & 17 & 114 & 176 & 99 & 297 & 12 & 23 \\
\hline 10 & 236 & 10 & 11 & 79 & 40 & 60 & 70 & 10 & 24 \\
\hline 11 & 22 & 18 & 20 & 64 & 67 & 62 & 86 & 16 & 33 \\
\hline 12 & 78 & 18 & 35 & 59 & 101 & 80 & 105 & 24 & 56 \\
\hline 13 & 2786 & 25 & 27 & 45 & 68 & 43 & 98 & 24 & 33 \\
\hline 14 & 138 & 60 & 24 & 186 & 175 & 53 & 77 & 14 & 55 \\
\hline 15 & 37 & 39 & 57 & 108 & 81 & 54 & 86 & 15 & 26 \\
\hline 16 & 85 & 31 & 34 & 173 & 66 & 146 & 67 & 16 & 39 \\
\hline 17 & 88 & 129 & 44 & 71 & 56 & 60 & 72 & 17 & 35 \\
\hline 18 & 15 & 47 & 35 & 89 & 72 & 82 & 63 & 14 & 38 \\
\hline 19 & 12 & 8 & 27 & 18 & 29 & 14 & 11 & 3 & 9 \\
\hline 20 & 153 & 14 & 19 & 152 & 61 & 78 & 79 & 32 & 46 \\
\hline 21 & 16 & 35 & 120 & 287 & 76 & 229 & 74 & 35 & 62 \\
\hline 22 & 1907 & 53 & 120 & 171 & 160 & 137 & 125 & 46 & 88 \\
\hline 23 & 1189 & 23 & 37 & 48 & 77 & 35 & 53 & 21 & 22 \\
\hline 24 & 1909 & 106 & 106 & 246 & 101 & 198 & 108 & 58 & 78 \\
\hline 25 & 411 & 72 & 139 & 60 & 68 & 79 & 68 & 219 & 51 \\
\hline 26 & 34 & 79 & 28 & 35 & 78 & 69 & 68 & 28 & 41 \\
\hline 27 & 56 & 391 & 503 & 180 & 165 & 146 & 122 & 46 & 68 \\
\hline 28 & 63 & 86 & 75 & 249 & 80 & 159 & 105 & 34 & 67 \\
\hline 29 & 32 & 107 & 96 & 49 & 64 & 53 & 91 & 37 & 45 \\
\hline 30 & 350 & 36 & 41 & 215 & 70 & 191 & 83 & 32 & 90 \\
\hline 31 & 4492 & 64 & 220 & 95 & 102 & 60 & 143 & 60 & 57 \\
\hline 32 & 434 & 76 & 129 & 178 & 161 & 119 & 100 & 58 & 85 \\
\hline 33 & 55 & 1150 & 608 & 248 & 83 & 164 & 96 & 77 & 298 \\
\hline 34 & 45 & 319 & 443 & 100 & 174 & 78 & 140 & 97 & 95 \\
\hline 35 & 2194 & 37 & 82 & 135 & 92 & 70 & 124 & 53 & 69 \\
\hline 36 & 12 & 367 & 331 & 92 & 110 & 30 & 102 & 106 & 58 \\
\hline 37 & 32 & 149 & 922 & 46 & 84 & 33 & 76 & 59 & 60 \\
\hline 38 & 63 & 32 & 73 & 29 & 96 & 42 & 81 & 438 & 46 \\
\hline 39 & 32 & 762 & 191 & 65 & 70 & 54 & 69 & 1741 & 74 \\
\hline 40 & 1418 & 68 & 457 & 129 & 110 & 71 & 81 & 350 & 63 \\
\hline 41 & 167 & 82 & 1069 & 245 & 126 & 140 & 76 & 363 & 66 \\
\hline 42 & 18 & 578 & 568 & 727 & 102 & 204 & 101 & 186 & 93 \\
\hline 43 & 21 & 362 & 126 & 73 & 85 & 64 & 64 & 2166 & 68 \\
\hline 44 & 8 & 591 & 2950 & 101 & 130 & 90 & 69 & 308 & 82 \\
\hline 45 & 10 & 4845 & 339 & 73 & 87 & 73 & 118 & 1608 & 68 \\
\hline 46 & 12 & 2917 & 240 & 47 & 76 & 72 & 94 & 1112 & 67 \\
\hline 47 & 6 & 940 & 230 & 39 & 53 & 52 & 66 & 595 & 54 \\
\hline 48 & 12 & 996 & 503 & 122 & 72 & 118 & 98 & 2120 & 154 \\
\hline 49 & 29 & 392 & 288 & 44 & 90 & 47 & 51 & 145 & 50 \\
\hline 50 & 192 & 30557 & 942 & 122 & 730 & 74 & 371 & 8733 & 541 \\
\hline 51 & 27 & 72 & 59 & 33 & 57 & 67 & 77 & 260 & 79 \\
\hline 52 & 29 & 347 & 239 & 35 & 111 & 39 & 61 & 300 & 79 \\
\hline 53 & 52 & 1798 & 261 & 84 & 102 & 52 & 110 & 876 & 170 \\
\hline 54 & 25 & 233 & 161 & 73 & 370 & 53 & 174 & 1694 & 628 \\
\hline
\end{tabular}




\begin{tabular}{|c|c|c|c|c|c|c|c|c|c|}
\hline 55 & 40 & 246 & 195 & 44 & 72 & 103 & 77 & 1484 & 113 \\
\hline 56 & 239 & 1164 & 478 & 82 & 115 & 259 & 118 & 1258 & 169 \\
\hline 57 & 116 & 673 & 509 & 176 & 221 & 97 & 90 & 1291 & 164 \\
\hline 58 & 24 & 51 & 33 & 32 & 81 & 22 & 28 & 52 & 25 \\
\hline 59 & 97 & 188 & 95 & 130 & 78 & 66 & 104 & 183 & 126 \\
\hline 60 & 94 & 896 & 101 & 107 & 111 & 222 & 93 & 1683 & 162 \\
\hline 61 & 64 & 319 & 78 & 18 & 115 & 68 & 72 & 1056 & 95 \\
\hline 62 & 78 & 143 & 51 & 35 & 299 & 53 & 67 & 159 & 66 \\
\hline 63 & 74 & 298 & 359 & 49 & 100 & 97 & 76 & 5438 & 369 \\
\hline 64 & 84 & 404 & 450 & 71 & 147 & 97 & 83 & 885 & 120 \\
\hline 65 & 294 & 492 & 775 & 63 & 163 & 81 & 109 & 562 & 107 \\
\hline 66 & 155 & 337 & 122 & 32 & 323 & 65 & 82 & 81 & 45 \\
\hline 67 & 233 & 1090 & 312 & 162 & 92 & 587 & 190 & 857 & 98 \\
\hline 68 & 208 & 586 & 674 & 70 & 281 & 181 & 163 & 1400 & 181 \\
\hline 69 & 208 & 557 & 806 & 77 & 161 & 120 & 115 & 626 & 124 \\
\hline 70 & 306 & 428 & 719 & 87 & 305 & 63 & 140 & 340 & 82 \\
\hline 71 & 210 & 601 & 242 & 27 & 281 & 81 & 108 & 198 & 68 \\
\hline 72 & 36 & 232 & 211 & 58 & 225 & 62 & 87 & 186 & 59 \\
\hline 73 & 74 & 250 & 514 & 78 & 133 & 55 & 83 & 272 & 118 \\
\hline 74 & 102 & 1781 & 366 & 64 & 315 & 100 & 117 & 305 & 116 \\
\hline 75 & 166 & 767 & 348 & 108 & 216 & 222 & 152 & 853 & 121 \\
\hline 76 & 136 & 610 & 443 & 65 & 305 & 115 & 123 & 643 & 130 \\
\hline 77 & 50 & 568 & 139 & 28 & 135 & 67 & 69 & 237 & 82 \\
\hline 78 & 56 & 342 & 207 & 58 & 225 & 91 & 105 & 320 & 82 \\
\hline 79 & 140 & 1659 & 2725 & 375 & 362 & 387 & 198 & 859 & 375 \\
\hline 80 & 124 & 522 & 204 & 99 & 362 & 217 & 186 & 484 & 77 \\
\hline 81 & 25 & 107 & 31 & 120 & 829 & 65 & 65 & 54 & 47 \\
\hline 82 & 16 & 77 & 73 & 84 & 1211 & 51 & 66 & 105 & 88 \\
\hline 83 & 48 & 331 & 130 & 150 & 5742 & 65 & 97 & 308 & 72 \\
\hline 84 & 31 & 152 & 109 & 103 & 1655 & 63 & 73 & 117 & 41 \\
\hline 85 & 23 & 220 & 91 & 86 & 1835 & 48 & 81 & 152 & 53 \\
\hline 86 & 26 & 128 & 69 & 81 & 1445 & 52 & 84 & 71 & 66 \\
\hline 87 & 37 & 161 & 149 & 527 & 414 & 64 & 80 & 57 & 97 \\
\hline 88 & 25 & 170 & 165 & 506 & 206 & 52 & 67 & 53 & 53 \\
\hline 89 & 45 & 264 & 220 & 402 & 733 & 62 & 88 & 69 & 92 \\
\hline 90 & 24 & 162 & 171 & 373 & 474 & 48 & 71 & 49 & 81 \\
\hline 91 & 23 & 119 & 74 & 194 & 555 & 53 & 85 & 34 & 62 \\
\hline 92 & 57 & 161 & 51 & 164 & 397 & 129 & 105 & 59 & 52 \\
\hline 93 & 31 & 132 & 174 & 146 & 575 & 92 & 93 & 49 & 149 \\
\hline 94 & 20 & 62 & 65 & 60 & 4765 & 40 & 60 & 24 & 71 \\
\hline 95 & 17 & 76 & 58 & 214 & 1489 & 49 & 67 & 29 & 56 \\
\hline 96 & 25 & 75 & 91 & 1113 & 1013 & 52 & 79 & 19 & 101 \\
\hline 2-FL & 4 & 510 & 20 & 18 & 68 & 44 & 55 & 18 & 53 \\
\hline NC & 1 & 6 & 6 & 14 & 54 & 38 & 79 & 4 & 31 \\
\hline
\end{tabular}

